\title{
Líneas de Retardo Real Para Conformación Óptica de Haces de Microondas
}

Tesis de Maestría

\section{Heinrich Sebastian RABAL}

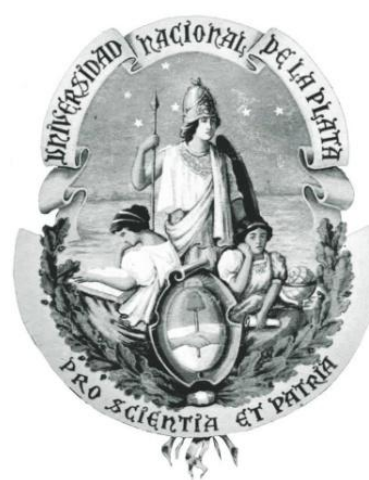

Presentada ante la Facultad de Ingeniería de la

Universidad Nacional de La Plata

como requisito para la obtención del grado académico de

\section{MAGÍSTER EN INGENIERÍA}

Dirección de tesis:
Director:
Dr. Pablo A. Costanzo Caso
Co-director:
Dr. Laureano A. Bulus Rossini

Jurado de tesis:
Dr. Enrique Spinelli
Dr. Gustavo Torchia
Dr. Javier Areta

La Plata, lunes 5 de diciembre de 2016. 

A mifamilia y amigas. 



\section{Índice general}

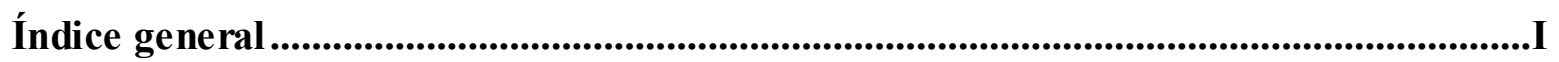

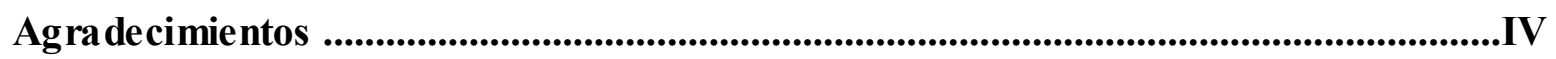

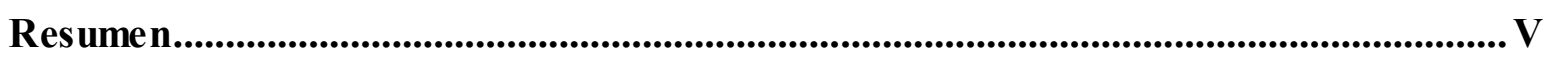

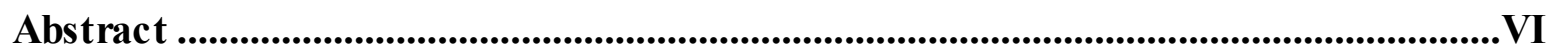

Abreviaciones ........................................................................................................................... VII

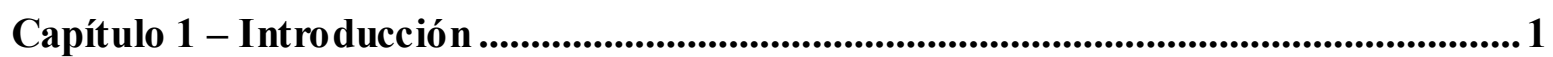

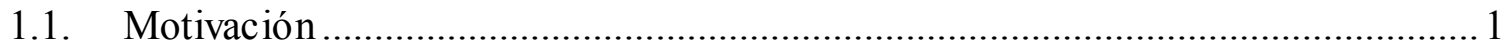

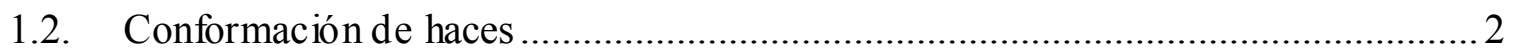

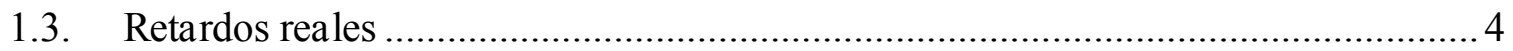

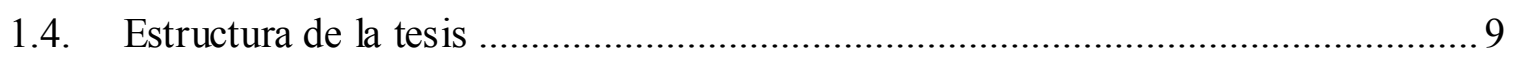

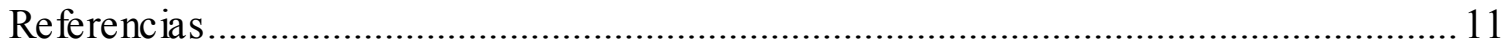

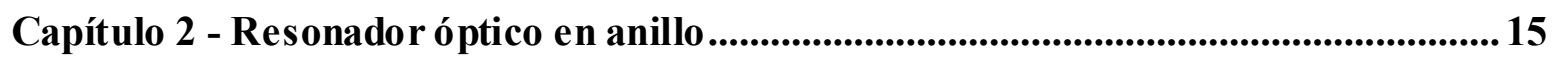

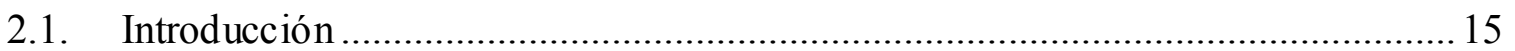

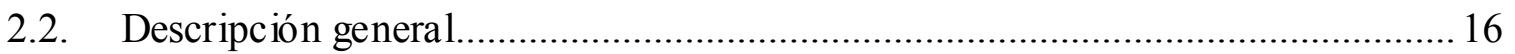

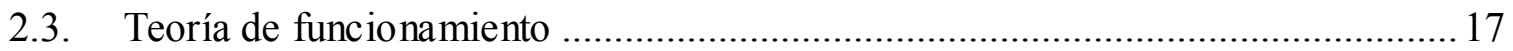

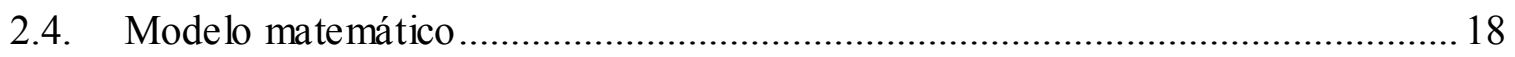

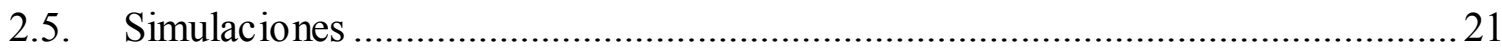

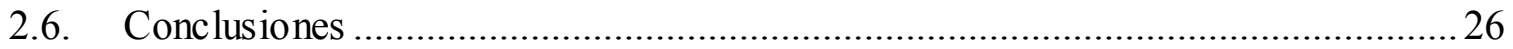

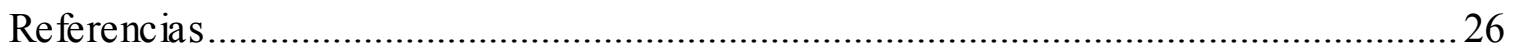

Capítulo 3 - Caracterización de la respuesta de un ORR ....................................................29

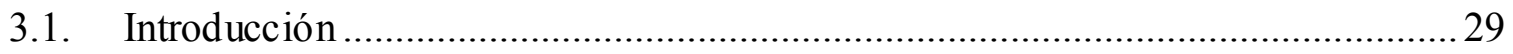

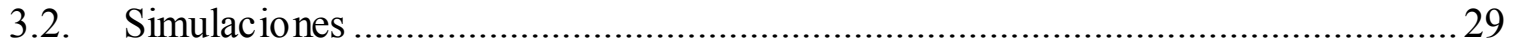

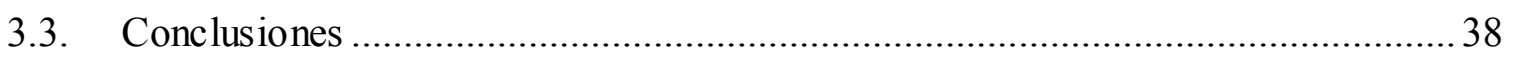

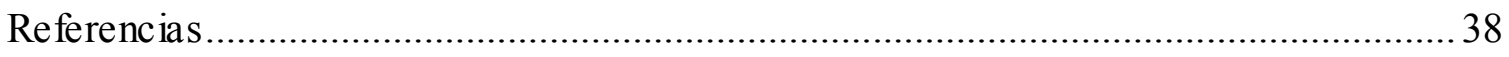

Capítulo 4 - Acopla miento crítico ……........................................................................41

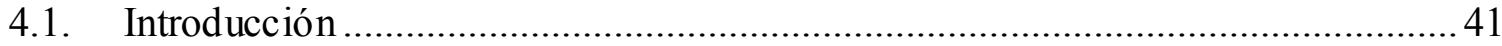

4.2. Variación fina del factor de acoplamiento ......................................................... 42

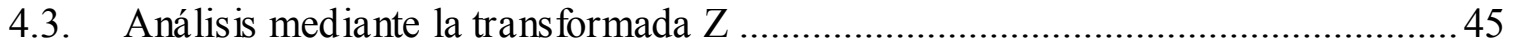

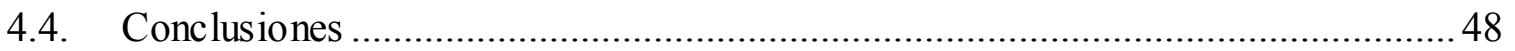

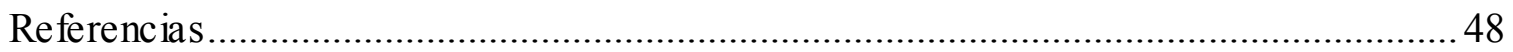


Capítulo 5 - ORR: Propagación de un pulso .........................................................................49

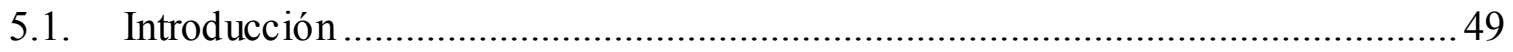

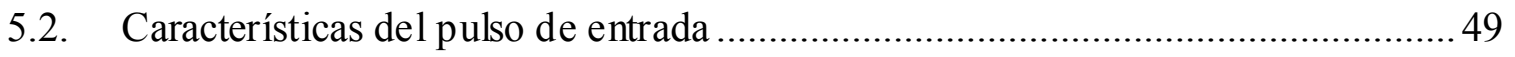

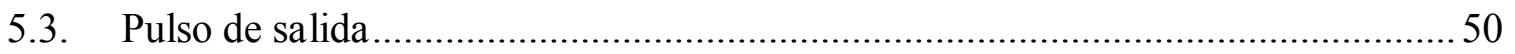

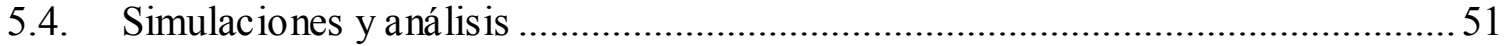

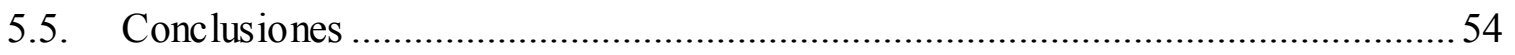

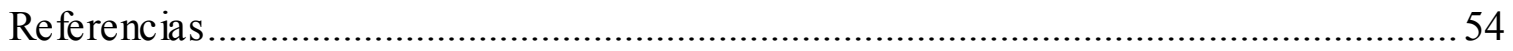

Capítulo 6 - ORR: Configuración de 4 pue rtos .........................................................57

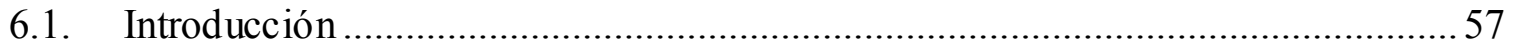

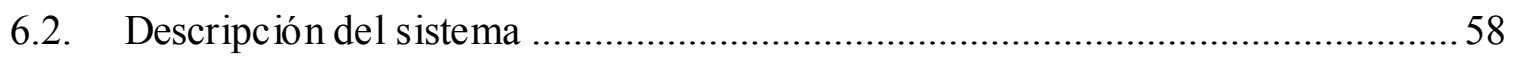

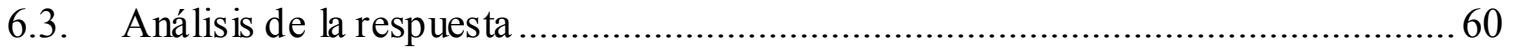

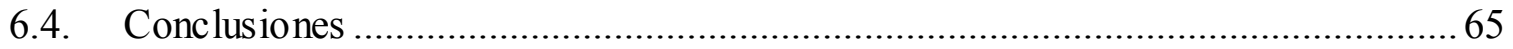

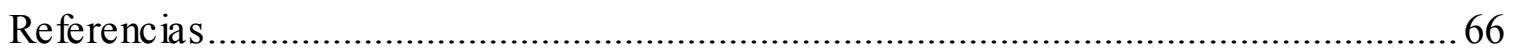

Capítulo 7 - ORR de 2 pue rtos: Conexión en casca da .............................................67

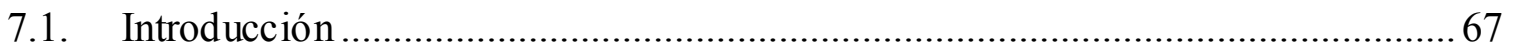

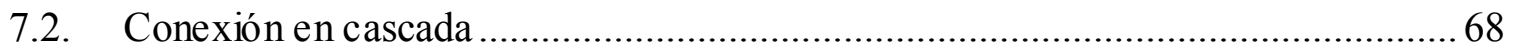

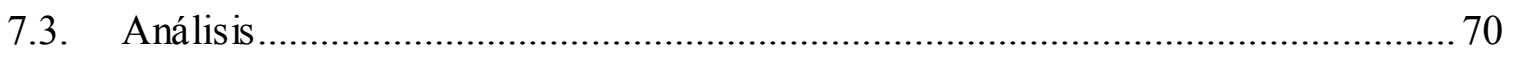

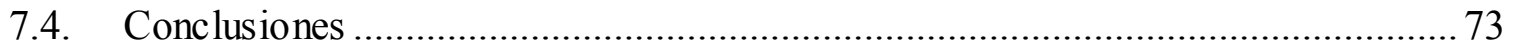

Capítulo 8 - ORR: conexión en paralelo .................................................................... 75

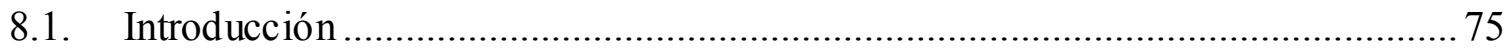

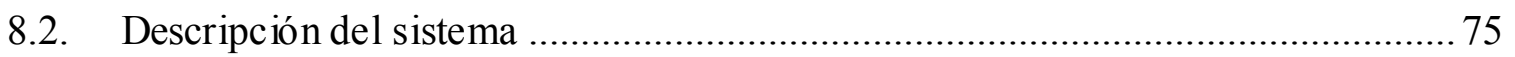

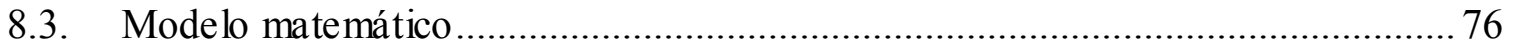

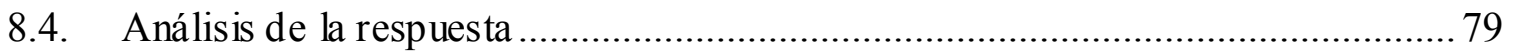

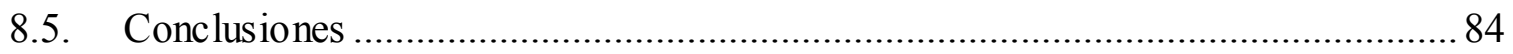

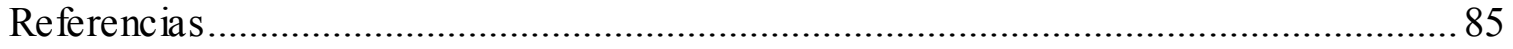

Capítulo 9 - ORR en cascada: algoritmo de control.................................................87

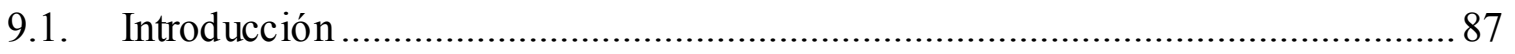

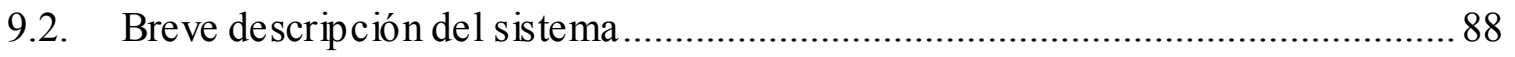

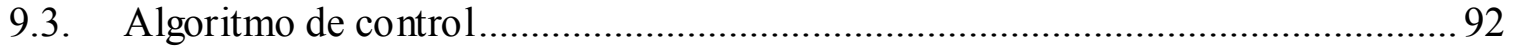

9.4. Presentación gráfica de los datos registrados ............................................... 101

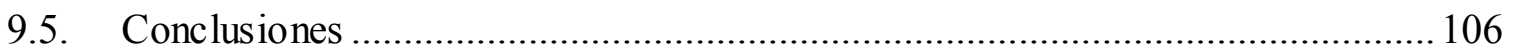


Capítulo 10 - Sistema de beamforming óptico basado en ORRs.......................................107

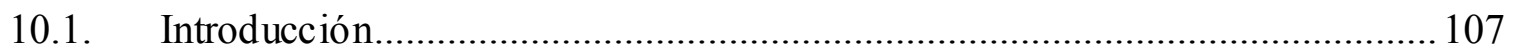

10.2. Análisis del desempeño de un enlace óptico analó gico .................................... 108

10.3. Sistema OBF basado en ORRs ................................................................ 123

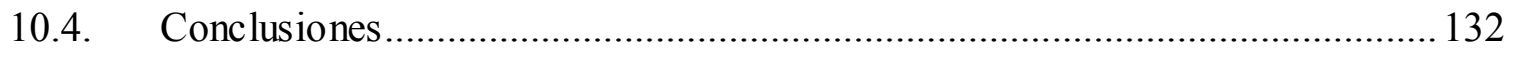

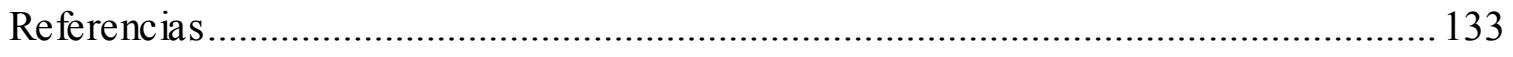

Conclusiones generales.......................................................................................................135

Apéndice A - Acoplador direccional ...................................................................................139

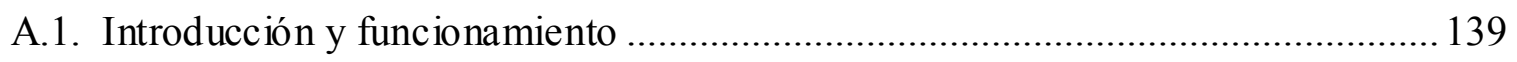

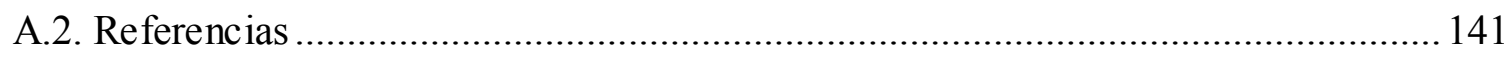

Apéndice B - Cálculo del valor pico y FWHM del retardo de grupo para un ORR .. 143 


\section{Agradecimientos}

Quiero expresar mi agradecimiento a todas las personas que hicieron posible este trabajo: a mis directores, el Dr. Pablo Costanzo Caso y el Dr. Laureano Bulus Rossini, por la insistencia y el apoyo que me dieron en todo momento; a mis compañeros del grupo de trabajo, el Ing. Leonardo Morbidel y el Ing. Alejandro Natoli, por su ayuda y su compañía en los diversos cursos, seminarios y congresos que compartimos; a los directivos y demás integrantes del Centro de Investigaciones Ópticas (CIOp), lugar en el que desarrollé mi trabajo, y en particular a todo el personal del Servicio de Corte Láser del CIOp.

Quiero agradecer también a los jurados de mi tesis, los doctores Enrique Spinelli, Gustavo Torchia y Javier Areta por sus comentarios y sugerencias, que me ayudaron a mejorar la versión final, y al director de la Escuela de Postgrado y Educación Continua (EPEC), el Dr. Sergio Giner, por su colaboración durante toda la maestría, y en especial en la instancia de defensa de la tesis.

Por último, pero no por ello menos importante, agradezco a la institución que financió mi trabajo como becario de postgrado, la Comisión de Investigaciones Científicas de la Provincia de Buenos Aires (CIC). 


\section{Resumen}

La conformación de haces por medios ópticos, Optical Beamforming (OBF), es una técnica que hace uso del procesamiento óptico o fotónico de señales de microondas para dar forma y dirección al patrón de radiación de un arreglo o conjunto de antenas.

En este trabajo se presenta el estudio de dispositivos fotónicos conocidos como Optical Ring Resonators (ORRs) que resultan aptos para generar los retardos reales necesarios en las señales de microondas que modulan una portadora óptica y luego alimentan el conjunto de antenas en un sistema de OBF.

Primeramente se obtiene un modelo matemático que describe la respuesta del dispositivo, y se analiza la influencia de cada uno de los parámetros que intervienen en él. Mediante simulaciones numéricas, se analiza la propagación de un pulso a través del resonador y se verifica su capacidad para generar retardos controlados.

Luego, con el fin de mejorar algunas características de la respuesta, se investigan distintas configuraciones y formas de conexión. A partir de este análisis, se elige la conexión en cascada de varios ORRs de dos puertos como la más apropiada para implementar líneas de retardo controlables que puedan luego ser aplicadas en un sistema de OBF. Se propone, entonces, una estrategia de control simple y eficaz para controlar los ORRs y así generar retardos con ciertas características deseadas, como valor medio y ripple dentro del ancho de banda de interés. Debido a su simplicidad, la estrategia propuesta puede ser implementada fácilmente en un dispositivo DSP o FPGA.

Por último, se analiza, mediante simulaciones numéricas, el desempeño de un sistema de OBF basado en las líneas de retardo estudiadas y la estrategia de control propuestas.

Los resultados obtenidos a lo largo del trabajo muestran las ventajas que presentan estos dispositivos fotónicos frente a su contraparte electrónica al ser utilizados en un sistema conformador de haz, relacionadas con la posibilidad de operar en grandes anchos de banda con variaciones mínimas en el ángulo de direccionamiento del sistema. 


\section{Abstract}

The beamforming by optical means, known as Optical Beamforming (OBF), is a technique that makes use of optical or photonic processing of microwave signals to shape and direct the radiation pattern of an array or set of antennas.

In this work, it is presented the study of photonic devices, known as Optical Ring Resonators (ORRs), suitable to generate the true-time delays of microwave signals, which are modulating an optical carrier and feed the antenna array in an OBF system.

First, a mathematical model describing the device response is obtained, and the influence of each of the parameters involved in it is analyzed. By means of numerical simulations, the propagation of a pulse through the resonator is analyzed, and its ability to generate controlled delays is verified.

Then, in order to improve some characteristics of the response, different configurations and connection types are investigated. From this analysis, the cascading of several twoports ORRs as the most appropriate to implement controllable delay lines that can then be applied to an OBF system is chosen. It is proposed, then, a simple and effective strategy for controlling the ORRs and generate delays with certain desired characteristics, such as mean value and ripple within the bandwidth of interest. Because of its simplicity, the proposed strategy can easily be implemented on a DSP or FPGA device.

Finally, by numerical simulations, the performance of an OBF system based on the studied delay lines and the proposed control strategy is analyzed.

Results show the advantages these photonic devices present over their electronic counterpart when used in a beamforming system, related to the possibility of operating in large bandwidths with minimal variations in the steering angle of the system. 


\section{Abreviaciones}

AC Corriente alterna (del inglés Alternating Current)

AE Elemento de antena (del inglés Antenna Element)

APD Fotodiodo de avalancha (del inglés Avalanche Photodiode)

BB Banda base (del inglés Baseband)

$\mathrm{BBoF} \quad$ Banda base sobre fibra (del inglés Baseband over Fiber)

BF Conformación de haces (del inglés Beamforming)

BLU Banda lateral única

BW Ancho de banda (del inglés Bandwidth)

$\mathrm{CW} \quad$ Onda continua (del inglés Continuous Wave)

DC Corriente continua (del inglés Direct Current)

DD Detección directa (del inglés Direct Detection)

DFB Realimentación distribuida (del inglés Distributed Feedback)

EDF A Amplificador de fibra dopada con erbio (del inglés Erbium Doped Fiber

Amplifier)

EOM Modulador electroóptico (del inglés Electrooptic Modulator)

FBG Red de Bragg grabada en fibra (del inglés Fiber Bragg Grating)

FFT Transformada rápida de Fourier (del inglés Fast Fourier Transform)

FO Fibra óptica

FSR Rango espectral libre (del inglés Free Spectral Range)

FWHM Ancho a mitad del máximo (del inglés Full Width at Half Maximum)

IF Frecuencia intermedia (del inglés Intermediate Frequency)

IFoF Frecuencia intermedia sobre fibra (del inglés Intermediate Frequency over

Fiber )

IM

Modulación de intensidad (del inglés Intensity Modulation)

LD Diodo láser (del inglés Laser Diode)

LED Diodo emisor de luz (del inglés Ligth Emitting Diode)

MEMS Sistemas microelectromecánicos (del inglés Microelectromechanical

Systems)

MF $\quad$ Modulador de fase 
MMF Fibra multimodo (del inglés Multimode Fiber)

MMIC Circuito integrado de microondas monolítico (del inglés Monolithic

Microwave Integrated Circuit)

MO Microondas

MZI Interferómetro Mach-Zehnder (del inglés Mach-Zehnder Interferometer)

OBF Conformación óptica de haces (del inglés Optical Beamforming)

OBFN Red de conformación óptica de haces (del inglés Optical Beamforming

Network)

OL Pérdidas ópticas (del inglés Optical Losses)

ORR Resonador óptico en anillo (del inglés Optical Ring Resonator)

PAA Arreglo de antenas (del inglés Phased Array Antennas)

PD Fotodiodo (del inglés Photodiode)

PIN Fotodiodo tipo PIN (estructura de semiconductor tipo P, Intrínseco y N)

PS Desplazador de fase (del inglés Phase Shifter)

RF Radiofrecuencia

RFoF Radiofrecuencia sobre fibra (del inglés)

RIN Ruido de intensidad relativa (del inglés Relative Intensity Noise)

RoF Radio sobre fibra (del inglés)

SMA Conector SMA (del inglés SubMiniature version A)

SMF Fibra monomodo (del inglés Single Mode Fiber)

TF Transformada de Fourier

TIA Amplificador de transimpedancia (del inglés Transimpedance Amplifier)

TTD Retardo real (del inglés True Time Delay)

WDM Multiplexado en longitud de onda (del inglés Wavelength Division Multiplexing) 


\section{Capítulo 1 - Introducción}

\subsection{Motivación}

Los sistemas de comunicación por radio son, hoy en día, una parte esencial en la vida cotidiana. Estos sistemas transmiten información a través de ondas electromagnéticas de frecuencias inferiores a las de la luz visible y están presentes en diversas aplicaciones como difusión de audio y/o video, telefonía, radar, navegación, comunicaciones satelitales, astronomía, etc.

La creciente demanda de servicios como internet de alta velocidad y difusión de contenido multimedia, así como la necesidad de que estos servicios estén disponibles en cualquier ubicación donde uno se encuentre, han motivado en los últimos años el desarrollo de sistemas de radio de banda ancha. Por ejemplo, durante un viaje en avión los pasajeros pueden querer acceder a servicios como televisión en directo, internet, etc. Sin embargo, el enlace de radio directo entre el avión y tierra no siempre está disponible, sobre todo cuando el avión se encuentra volando sobre el mar en vuelos intercontinentales, y debe recurrirse a un enlace a través de un satélite. Se pone de manifiesto, entonces, la necesidad de desarrollar sistemas de comunicaciones de banda ancha capaces de satisfacer estos servicios y que sean sensibles a la dirección de las señales a recibir y transmitir.

En los sistemas como el del ejemplo anterior, y en otros como los utilizados en radioastronomía, la potencia de señal recibida es muy baja (del orden de $-100 \mathrm{dBm}$ y hasta el límite impuesto por la cifra de ruido), y por lo tanto se requieren antenas de alta ganancia y sensibles a la dirección para la recepción de la señal. 
Un ejemplo de antena sensible a la dirección es una antena de plato parabólico, que puede ser dirigida mecánicamente para orientar su haz principal en diferentes direcciones. Sin embargo, el movimiento mecánico de este tipo de antenas limita su precisión de ajuste y velocidad. En el caso del avión, el uso de este tipo de antenas trae aparejados inconvenientes debido a su gran peso, tamaño y el efecto de arrastre aerodinámico cuando se monta en la parte superior del avión. Este tipo de problemas motivó el desarrollo de ciertas técnicas de conformación de haces, las cuales se explican en la siguiente sección.

\subsection{Conformación de haces}

La conformación de haces, Beamforming (BF) en inglés, es una técnica o conjunto de técnicas que se utilizan para dar forma y dirección al patrón de radiación de una antena. En este caso, en lugar de utilizar una antena de plato parabólico simple se hace uso de lo que se conoce como Phased Array Antennas (PAA). Un PAA es un conjunto de elementos de antena (Antenna Elements (AEs)) ubicados de manera ordenada uno próximo al otro. $\mathrm{Su}$ patrón de radiación está determinado por la geometría del conjunto, así como la amplitud y la relación entre las fases relativas de las señales que alimentan los AEs. De esta manera, eligiendo adecuadamente la relación de amplitudes y fases, puede modificarse el patrón de radiación efectivo del conjunto de manera que se intensifique en una dirección dada y se suprima en las direcciones no deseadas $[1,2]$. Una de las ventajas del BF es que permite orientar el patrón de radiación sin realizar ningún movimiento mecánico.

La intensificación del patrón de radiación del conjunto en la dirección deseada produce un haz principal. En la Fig. 1.1 se ilustra el efecto del cambio en la orientación del haz principal: en línea negra punteada se muestra el haz principal de un PAA en dirección de $0^{\circ}$, mientras que en línea roja sólida se muestra el haz direccionado a $-30^{\circ}$ con respecto al caso anterior. 


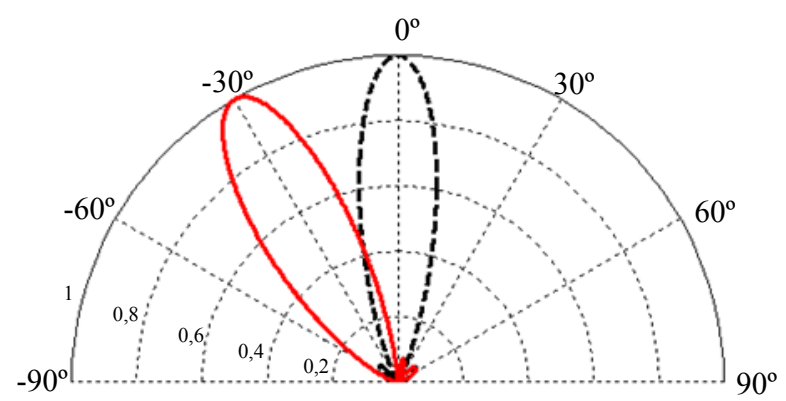

Figura 1.1: Patrón de radiación y cambio de dirección.

Existen varias geometrías que puede adoptar el conjunto de antenas, y también distintas técnicas de procesamiento de señal avanzadas para lograr patrones particulares de radiación efectivo [3,4]. El PAA más simple es uno lineal y consiste en un conjunto de AEs idénticos igualmente espaciados a lo largo de una sola línea, como se muestra en los ejemplos de la Fig. 1.2 para el caso de un conjunto de cuatro elementos.

Un PAA puede utilizarse tanto en un sistema transmisor, para direccionar la señal que se desea transmitir, como en un sistema receptor, para recibir las señales provenientes de determinada dirección. En la Fig. 1.2 se muestra un ejemplo de cada caso.

En un sistema transmisor, como el de la Fig. 1.2(a), la señal a transmitir se divide previamente en tantas partes como elementos de antena componen el PAA. Las señales resultantes deben ser acondicionadas antes de alimentar los AEs, y para ello se requiere un circuito dedicado, indicado en la figura como beamformer, que controla tanto las amplitudes como las fases de las mismas. Convencionalmente, este circuito se implementa en el dominio eléctrico pero también puede realizarse en el dominio óptico, constituyendo un Optical Beamformer (OBF).

En la Fig. 1.2(b) se muestra un sistema receptor. La salida del sistema es la suma de las señales (corrientes) de todos los AEs, las cuales se suman constructivamente cuando están en fase y destructivamente cuando están en contrafase. En este caso, el beamformer se encarga de "ecualizar" las fases de manera que la señal que provenga de la dirección deseada se intensifique y cualquier otra señal resulte atenuada. 


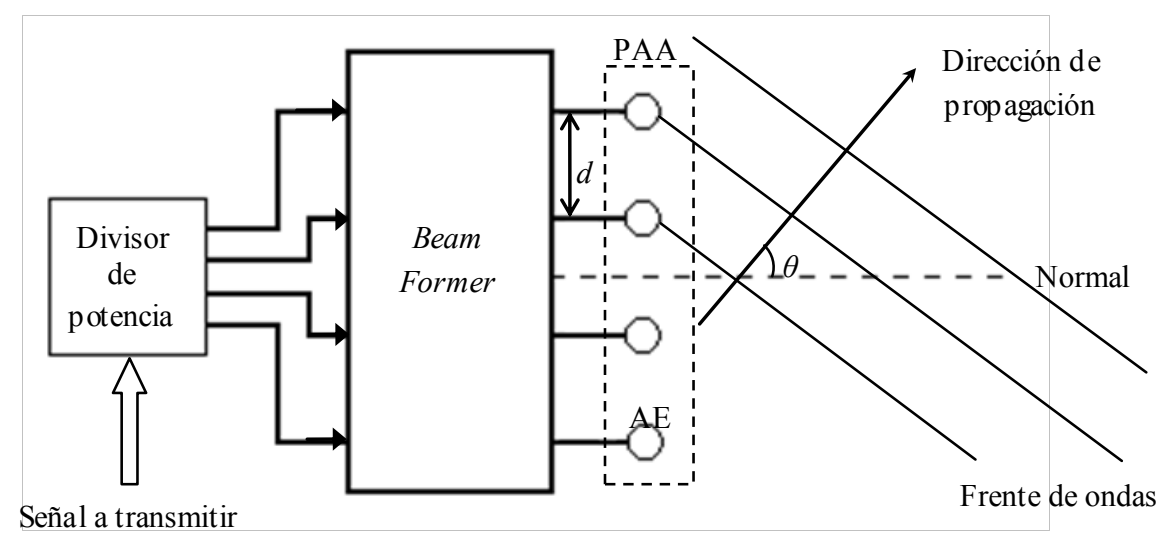

(a)

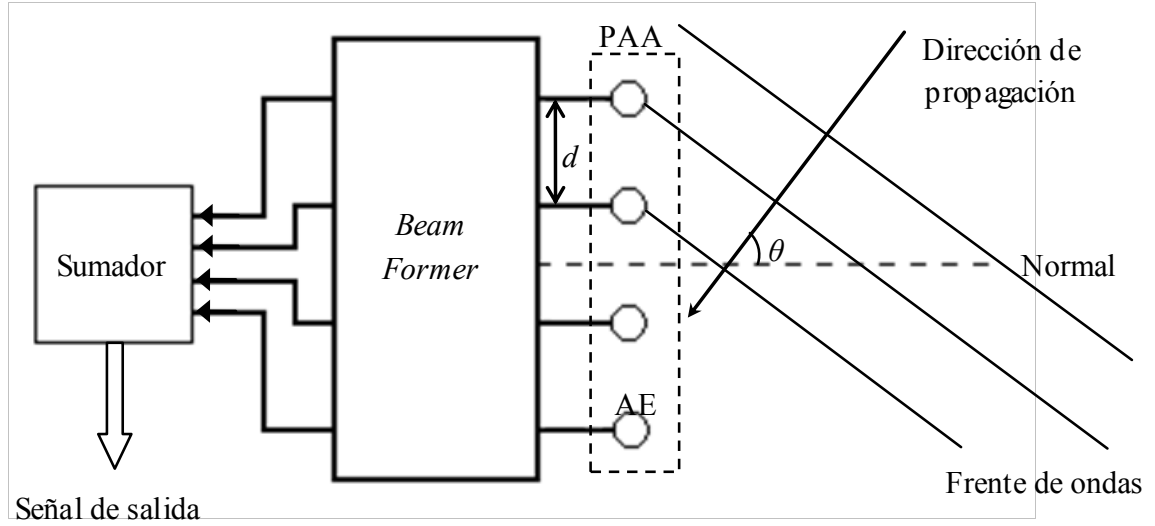

(b)

Figura 1.2: PAA lineal: (a) en un sistema transmisor; (b) en un sistema receptor.

Ya sea que se trate de un sistema transmisor o receptor, si el haz principal del patrón de radiación forma un ángulo $\theta$ con la normal al PAA, el desplazamiento de fase entre las señales que alimentan (o que provienen de) dos AEs adyacentes está dado por la Ec. (1.1):

$$
\Delta \varphi=2 \pi \frac{d}{\lambda} \cdot \operatorname{sen}(\theta)
$$

donde $d$ es la distancia entre los AEs, y $\lambda$ la longitud de onda de la señal a transmitir (o recibir). De esta manera, el beamformer debe proporcionar los desplazamientos de fase $\Delta \varphi$ adecuados para que el patrón de radiación del PAA se intensifique en la dirección $\theta$.

\subsection{Retardos reales}

Si en la Ec. (1.1) se sustituye la frecuencia por la de longitud de onda, $\lambda=\mathrm{c} / f$ se obtiene la Ec. (1.2) y se hace evidente la limitación de este método a señales de banda estrecha, es 
decir aquellas cuyo ancho de banda sea mucho menor que la frecuencia portadora. Esto se debe a que el desplazamiento de fase necesario para direccionar un cierto ángulo depende de la frecuencia. Así, si el sistema transmite señales de gran ancho de banda, las distintas componentes frecuenciales se irradian en diferentes direcciones y el haz sufre una desviación, o squint en inglés, que puede traducirse como "estrabismo" del haz.

$$
\begin{gathered}
\Delta \varphi=2 \pi \frac{d \cdot f}{c} \cdot \operatorname{sen}(\theta) \\
\Delta t=\frac{d}{c} \cdot \operatorname{sen}(\theta)
\end{gathered}
$$

En un sistema receptor, el beam squint produce una distorsión lineal de la señal que se recibe debido a que la deformación del patrón de radiación se traduce en diferente atenuación para distintas frecuencias.

Por otro lado, de la Ec. (1.2) se desprende que si el desplazamiento de fase $\Delta \varphi$ se hace proporcional a la frecuencia, se evita el beam squint y se incrementa el ancho de banda del PAA. En otras palabras, para que todas las componentes de frecuencia se irradien en la misma dirección, en vez de aplicar un desplazamiento de fase debe aplicarse un retardo temporal entre las señales de los AEs, como indica la Ec. (1.3). Se dice que los sistemas que realizan esta operación aplican un retardo de tiempo real (True Time Delay (TTD)) a las señales, como se esquematiza en la Fig. 1.3.

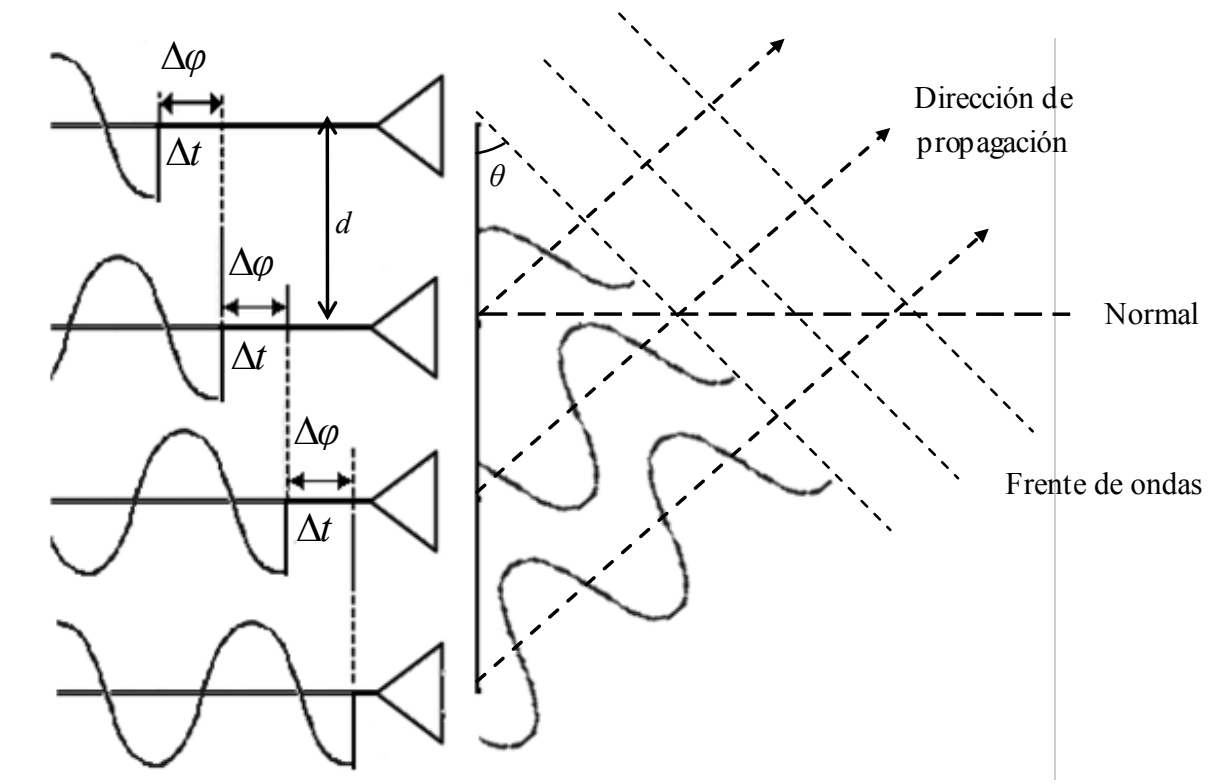

Figura 1.3: Direccionamiento del haz por medio de TTDs. 
Se observa que si los retardos relativos entre las señales que alimentan los AEs son adecuados, las ondas se encontrarán en fase para una dada dirección y no para las demás. El valor de los retardos relativos necesarios para direccionar el haz principal con un ángulo $\theta$ está dado por la Ec. (1.3) y depende de la distancia entre AEs, $d$, y la velocidad de propagación, $c$.

\section{Retardos reales: implementación}

Dependiendo de la banda de frecuencias, existen varios métodos para realizar un retardo como los que se requieren en el beamformer. Entre los métodos electrónicos de microondas que suelen usarse para implementar TTDs, pueden mencionarse las líneas de transmisión de cable coaxial, aunque estos tienen la desventaja de las grandes pérdidas y peso. Se pueden utilizar también líneas de microtira en circuitos impresos, contrarrestando las pérdidas con amplificadores. Las líneas de retardo, en estos casos, se implementan típicamente usando pequeños conmutadores que seleccionan entre líneas de distintas longitudes para generar retardos variables [5].

Por otro lado, los avances en la fabricación de dispositivos semiconductores han permitido obtener retardos en espacios muy reducidos, usando sistemas microelectromecánicos (Microelectromechanical Systems (MEMS)) [6-8] y circuitos integrados de microondas monolíticos (Monolithic Microwave Integrated Circuits (MMICs)) [9,10].

\section{Retardos reales por medios fotónicos}

La fotónica de microondas es un área que estudia la interacción entre las microondas y las ondas ópticas en aplicaciones como radar, comunicaciones, instrumentación y redes de sensores, entre otras. En general, comprende a las técnicas utilizadas para generar, procesar y distribuir señales de microondas por medios fotónicos.

En particular, un OBF es un dispositivo que hace uso del procesamiento fotónico de señales para generar los retardos temporales controlados en las señales que alimentan el PAA. En comparación con su contraparte eléctrica, el OBF tiene ventajas tales como la compacticidad, el peso ligero, bajas pérdidas, la independencia de la frecuencia, un amplio ancho de banda instantáneo, y la inmunidad inherente a las interferencias electromagnéticas. 
Existen diversos métodos fotónicos que pueden emplearse para hacer el procesamiento de la señal en el dominio óptico, como: conmutación de guías ópticas [11-14], líneas de retardo basadas en redes de Bragg uniformes, con chirp y superestructuradas [15-22], TTD por propagación en medios dispersivos [23-27], y otros [28-34]. A continuación, se describe un sistema que utiliza dispositivos de fibra óptica conocidos como redes de Bragg uniformes.

\section{Conformador de haces con redes de Bragg}

Algunos sistemas OBF investigados en los últimos años hacen uso de redes de Bragg uniformes grabadas en fibra (FBGs) para implementar las líneas de retardo [35,36]. Las FBGs son dispositivos de fibra óptica que se obtienen realizando un grabado periódico del índice de refracción en el núcleo de la misma, y se comportan como reflectores para cierta longitud de onda, $\lambda_{\mathrm{B}}$, que depende de las características constructivas del dispositivo.

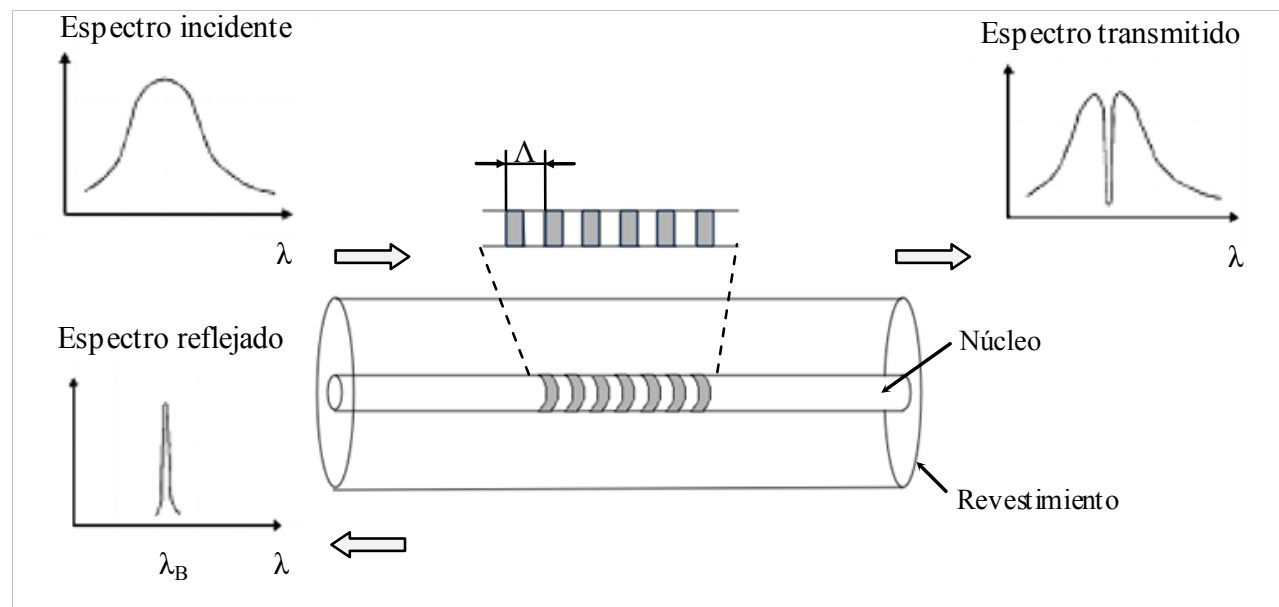

Figura 1.4: Esquema de una red de Bragg uniforme grabada en fibra óptica.

$$
\lambda_{B}=2 \cdot n_{e f} \cdot \Lambda
$$

En la Fig. 1.4 se presenta el esquema de una FBG y se indica el período $\Lambda$ del grabado, que determina, junto con el índice de refracción efectivo $n_{\mathrm{ef}}$, la longitud de onda de Bragg, $\lambda_{\mathrm{B}}$, que se refleja (Ec. (1.4)). El ancho de banda de $3 \mathrm{~dB}$ típico de una FBG es delorden de $0.2 \mathrm{~nm}$, que equivale aproximadamente a $25 \mathrm{GHz}$ en frecuencia. 
A modo de ejemplo, en la Fig. 1.5 se muestra el esquema de un posible OBF basado en FBGs en modo transmisor. El sistema consta de un PAA lineal de cuatro elementos separados entre sí una distancia $d$, un láser sintonizable en longitudes de onda y un modulador electroóptico (Electrooptic Modulator (EOM)) que se encarga de modular la portadora óptica con la señal de radiofrecuencias (RF) o microondas (MO) a transmitir. La señal resultante es dividida por medio de un divisor de potencia y dirigida mediante circuladores hacia las distintas líneas de retardo, que consisten en fibras ópticas con redes de Bragg ubicadas de manera particular. La luz que se refleja en las FBGs de líneas adyacentes recorre distintas distancias hasta incidir en los fotodiodos (PDs) detectores. Esta diferencia en las distancias recorridas se traduce en un retardo temporal relativo entre las señales que modulan la portadora óptica. Una vez detectadas, las señales pueden ser amplificadas antes de transmitirse mediante los AEs.

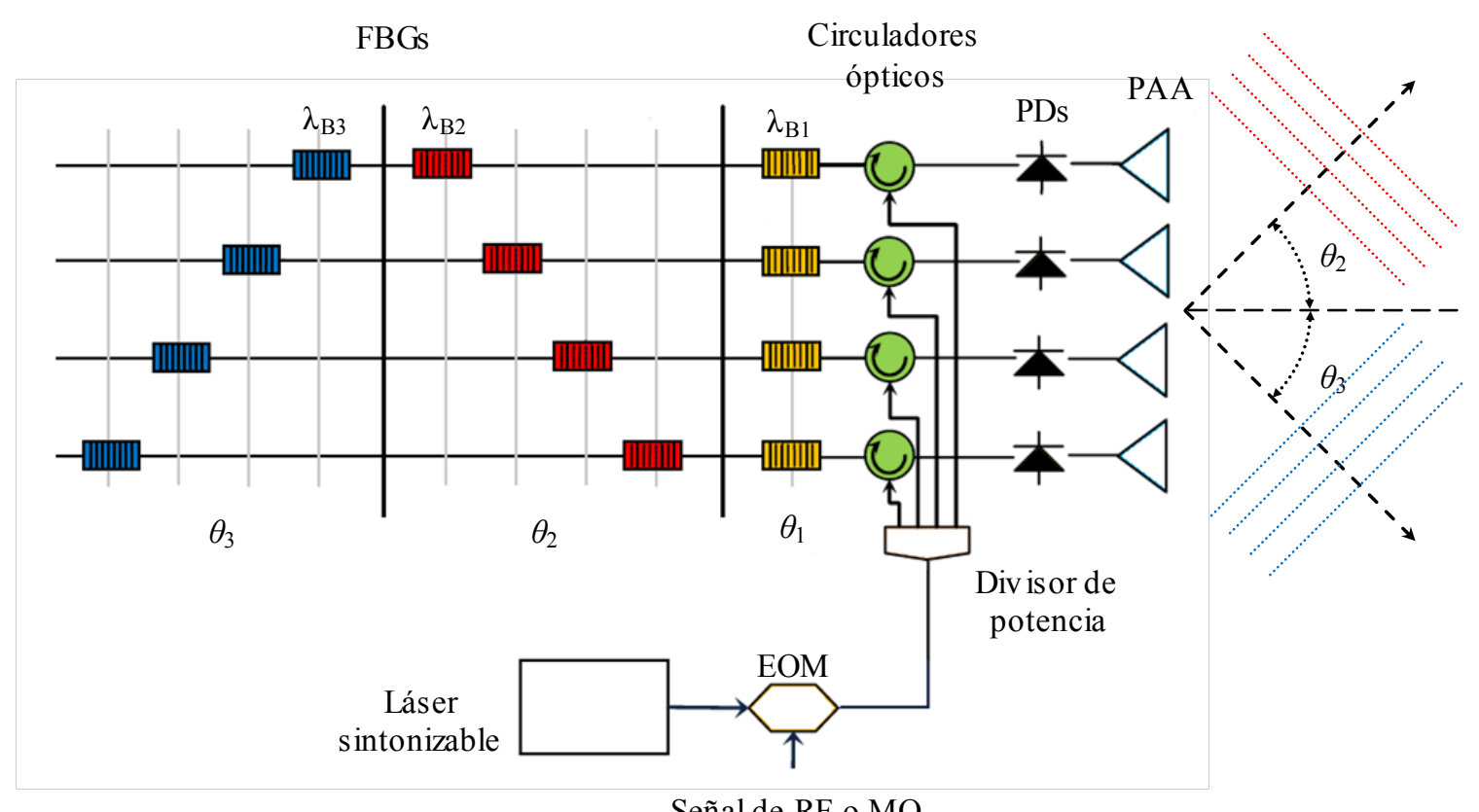

Figura 1.5: Ejemplo: Sistema OBF basado en redes de Bragg uniformes.

En este sistema, el ángulo de direccionamiento del haz sólo puede tomar valores discretos, cada uno de los cuales está determinado por las ubicaciones relativas de las FBGs, que se agrupan de acuerdo a su longitud de onda de Bragg. De esta manera, la 
cantidad de FBGs utilizadas es directamente proporcional al número de ángulos que el sistema puede direccionar.

Por otro lado, en los últimos años se han investigado ciertos dispositivos fotónicos aptos para generar retardos en las señales del OBF, conocidos como Optical Ring Resonators (ORRs) [37-43], que consisten, como su nombre lo indica, en resonadores ópticos con forma de anillo. En el Capítulo 2 se describen estos dispositivos con más detalle, pero aquí cabe mencionar que son aptos para generar retardos controlados en forma continua, lo que se traduce en ángulos de direccionamiento también continuos. Además, con las técnicas de fabricación adecuadas, las líneas de retardo basadas en ORRs pueden implementarse de dimensiones mucho menores a las del ejemplo anterior.

Esta tesis está orientada al estudio de los retardos reales producidos mediante ORRs en señales de MO, con el fin de implementar líneas de retardo que puedan luego ser aplicadas en un sistema conformador de haz. Las señales de microondas consideradas en este trabajo pertenecen a la banda $\mathrm{X}$ (entre 8,2 y 12,4 GHz), y los anchos de banda entre 1 y $2 \mathrm{GHz}$. Con respecto a la potencia de la portadora óptica, con el fin de evitar los efectos no lineales que se hacen evidentes en las guías de onda para grandes potencias, se consideran valores del orden de $0 \mathrm{dBm}(1 \mathrm{~mW})$.

\subsection{Estructura de la tesis}

La tesis está estructurada de la siguiente manera: En elCapítulo 2 presenta un resonador óptico en anillo y se describe su teoría de funcionamiento. En el Capítulo 3 se estudia, mediante simulaciones numéricas, la influencia de distintos parámetros en la respuesta del dispositivo. En el Capítulo 4 se analiza una condición particular de funcionamiento conocida como condición de acoplamiento crítico y se diferencian dos zonas de trabajo. En el Capítulo 5 se simula la propagación de un pulso a través de un ORR para diferentes condiciones de acoplamiento del mismo. En el Capítulo 6 se estudia una configuración de ORR ligeramente diferente, con dos puertos de entrada y dos de salida, que permite su aplicación como selector de canales multiplexados en longitud de onda. En el Capítulo 7 se presenta una topología que emplea varios ORRs conectados en cascada con el fin de mejorar las características de la respuesta, en particular el ancho de banda. En el Capítulo 8 se investiga una conexión alternativa, esta vez en paralelo, y se realizan las observaciones 
pertinentes con respecto a la posibilidad de utilizarla para implementar retardos de banda ancha. En el Capítulo 9 se propone una estrategia de control para una línea de retardo compuesta por ORRs en cascada y se presentan algunos resultados que muestran sus bondades. En el Capítulo 10 se analiza el patrón de radiación de un sistema OBF que emplea las líneas de retardo y la estrategia de control presentadas en el capítulo anterior. Finalmente, en el Capítulo 11 se presentan las conclusiones y perspectivas de trabajo para el futuro.

Durante el período de desarrollo de la Tesis de Maestría fueron presentados, en colaboración, los siguientes trabajos:

Sebastian Rabal, Pablo A. Costanzo Caso, "Desviaciones del patrón de irradiación de un OBF" Póster en: Encuentro de Estudiantes de Óptica y Fotofísica (EEOF) - Taller de Óptica y Fotofísica (TOPFOT) 2012, 21-24 Mayo 2012, Centro Científico Tecnológico CONICET La Plata.

Pablo A. Costanzo Caso, Sebastian Rabal, Emanuel Paulucci, Alejandro Giordana, y Laureano A. Bulus Rossini, "Practical impairments in FBG-based rue time delays," Artículo completo en: Proceeding of Latin American Optics and Photonics Conference (LAOP) 2012, 13-15 de Noviembre, Sao Sebastiao, Brasil. pp.1-3.

Sebastian Rabal, Laureano A. Bulus Rossini, Pablo A. Costanzo Caso, "Resonador óptico en anillo y su aplicación en la implementación de líneas de retardo controlables" Póster en: Encuentro de Estudiantes de Óptica y Fotofísica (EEOF) - Taller de Óptica y Fotofísica (TOPFOT) 2013, 20-23 Mayo 2013, Departamento de Física Facultad de Ciencias Exactas y Naturales, Universidad de Buenos Aires, Ciudad Autónoma de Buenos Aires.

Sebastian Rabal, Laureano A. Bulus Rossini, Pablo A. Costanzo Caso, "Análisis de un resonador óptico en anillo y su aplicación en el desarrollo de líneas de retardo". Artículo completo de 6 páginas en: XV Reunión de Trabajo en Procesamiento de la Información y Control (RPIC) 2013, 16-20 Septiembre 2013, San Carlos de Bariloche, Río Negro, Argentina.

Sebastian Rabal, Laureano A. Bulus Rossini y Pablo A. Costanzo Caso, "Sintonización de Líneas de Retardo de Banda Ancha Basadas en ORRs". Artículo completo de 6 páginas en ARGENCON 2014, 11-13 de junio de 2014, San Carlos de Bariloche, Río Negro, Argentina.

Sebastian Rabal, Laureano A. Bulus Rossini y Pablo A. Costanzo Caso, "Control Strategy of True Time Delay Lines," Taylor \& Francis, Fiber and Integrated Optics, November 2016, in press. 


\section{Referencias}

[1] H. L. Van Trees, Optimum Array Processing. Part IV, Wiley 2002.

[2] W. Liu \& S. Weiss, Wideband Beamforming, Concepts and Technics, Wiley 2010

[3] R. C. Hansen, Phased array Antennas, Second Edition, Wiley 2009.

[4] Robert J. Mailloux, Phased Array Antenna Handbook, Second Edition, Artech House 2005.

[5] Nemai Chandra Karmakar (Editor), Handbook of Smart Antennas for RFID Systems, Wiley 2010.

[6] N. S. Barker and G. M. Rebeiz, "Distributed MEMS True-Time Delay Phase Shifters and Wide-Band Switches," IEEE Transactions on Microwave Theory and Thechniques, Vol. 46, No. 11, November 1998, pp. 1881-1890.

[7] M. Kim, J. B. Hacker, R. E. Mihailovich, and J. F. DeNatale, "A DC-to-40 GHz FourBit RF MEMS True-Time Delay Network," IEEE Microwave and Wireless Components Letters, Vol. 11, No. 2, February 2001, pp. 56-58.

[8] S. Ibrahim, G. Szczepkowski, R. Farrell, "Wideband MEMS Switched Delay Lines with High Phase Linearity," 21st IEEE International Conference on Electronics, Circuits and Systems (ICECS), 2014.

[9] J. G. Willms, A. Ouacha, L. de Boer, and F. E. van Vliet, "A wideband GaAs 6-bit truetime delay MMIC employing on-chip digital drivers," in 2000 30th European Microwave Conference, Oct. 2-5, 2000.

[10] X. Lan, M. Kintis, C. Hansen, W. Chan, G. Tseng, and M. Tan, “A Simple DC to 110 GHz MMIC True Time Delay Line," IEEE Microwave and Wireless Components Letters, Vol. 22, No. 7, July 2012, pp. 369-371.

[11] Y. Chen, K. Wu, F. Zhao, G. Kim, R. T. Chen, "Reconfigurable True-Time Delay for Wideband Phased-Array Antennas," Emerging Optoelectronic Applications, edited by Ghassan E. Jabbour, Juha T. Rantala, Proceedings of SPIE Vol. 5363 (SPIE, Bellingham, WA, 2004), pp. 125-130.

[12] B. Jung, D. Kim, I. Jeon, S. Shin, and H. Kim, "Optical True Time-Delay Beamformer Based on Microwave Photonics for Phased Array Radar”, IEEE 3rd International AsiaPacific Conference on Synthetic Aperture Radar (APSAR), 2011.

[13] J. Xie, L. Zhou, Z. Li, J. Wang, and J. Chen, "Seven-bit reconfigurable optical true time delay line based on silicon integration," Optics Express, Vol. 22, No. 19, 22 September 2014, pp. 22707-22715.

[14] L. Yaron, R. Rotman, S. Zach, and M. Tur, "Photonic Beamformer Receiver With Multiple Beam Capabilities," IEEE Photonics Technology Letters, Vol. 22, No. 23, December 1, 2010, pp. 1723-1725.

[15] H. Zmuda, R. A. Soref, P. Payson, S. Johns, and E. N. Toughlian, "Photonic Beamformer for Phased ArrayAntennas Using a Fiber Grating Prism," IEEE Photonics Technology Letters, Vol. 9, No. 2, February 1997, pp. 241-143. 
[16] R. A. Minasian, and K. E. Alameh, "Optical-Fiber Grating-Based Beamforming Network for Microwave Phased Arrays", IEEE Transactions on Microwave Theory and Techniques, Vol. 45, No. 8, August 1997, pp. 1513-1518.

[17] J. Yao, J. Yang, and Y. Liu, “Continuous True-Time-Delay Beamforming Employing a Multiwavelength Tunable Fiber Laser Source," IEEE Photonics Technology Letters, Vol. 14, No. 5, May 2002, pp. 687-689.

[18] Y. Liu, J. Yang, and J. Yao, "Continuous True-Time-Delay Beamforming for Phased Array Antenna Using a Tunable Chirped Fiber Grating Delay Line”, IEEE Photonics Technology Letters, Vol. 14, No. 8, August 2002, pp. 1172-1174.

[19] J.Yang, J. Yao, Y. Liu, and S. Chuan Tjin, "Continuous true-time-delay beamforming employing a tunable multiwavelength fiber ring laser source with equally increased or decreased wavelength spacing", Optical Engineering, Vol. 42 No. 1, January 2003, pp. 239-244.

[20] S. Granieri, M. Jaeger, and A. Siahmakoun, "Multiple-Beam Fiber-Optic Beamformer With Binary Array of Delay Lines," Journal of Lightwave Technology, Vol. 21, No. 12, December 2003, pp. 3262-3272.

[21] B. Jung and J. Yao, “A Two-Dimensional Optical True Time-Delay Beamformer Consisting of a Fiber Bragg Grating Prism and Switch-Based Fiber-Optic Delay Lines," IEEE Photonics Technology Letters, Vol. 21, No. 10, May 15, 2009, pp. 627 629.

[22] S. Blais, and J. Yao, 'Photonic True-Time Delay Beamforming Based on Superstructured Fiber Bragg Gratings With Linearly Increasing Equivalent Chirps," Journal of Lightwave Technology, Vol. 27, No. 9, May 1, 2009, pp. 1147-1154.

[23] E. Udvary, T. Berceli, "New Microwave / Millimeter Wave True Time Delay Device Utilizing Photonic Approaches," Proceedings of the 40th European Microwave Conference, 28-30 September 2010, Paris, France, pp. 121-124.

[24] H. Lee, H. Jeon, J. Jung, “Optical True Time-Delay Beam-forming for Phased Array Antenna Using a Dispersion Compensating Fiber and a Multi-wavelength Laser," IEEE 4th Annual Caneus Fly by Wireless Workshop (FBW), 2011.

[25] H. Jeon, J. Jeong, H. Lee, “Optical True Time-Delay for Phased-Array Antenna System Using Dispersion Compensating Module and a Multi-wavelength Fiber Laser," 17th Opto-Electronics and Communications Conference (OECC 2012) Technical Digest, July 2012, Busan, Korea.

[26] P. Wu, S. Tang, and D. E. Raible, “A prototype high-speed optically-steered X-band phased array antenna," Optics Express, Vol. 21, No. 26, 30 December 2013, pp. 32599-32604.

[27] X. Ye, F. Zhang, and S. Pan, "Optical true time delay unit for multibeamforming," Optics Express, Vol. 23, No. 8, 20 Apr 2015, pp. 10002-10008.

[28] M. V. Drummond, P. P. Monteiro, and R. N. Nogueira, "Photonic True-Time Delay Beamforming Based on Polarization-Domain Interferometers," Journal of Lightwave Technology, Vol. 28, No. 17, September 1, 2010, pp. 2492- 2498. 
[29] D. T. K. Tong and M. C. Wu, "Multiwavelength Optically Controlled Phased-Array Antennas," IEEE Transactions on Microwave Theory and Techniques, Vil. 46, No. 1, January 1998, pp. 108-115.

[30] M. Y. Chen, "Hybrid Photonic True-Time Delay Modules for Quasi-Continuous Steering of 2-D Phased-Array Antennas," Journal of Lightwave Technology, Vol. 31, No. 6, March 15, 2013, pp. 910-917.

[31] W. Xue and J. Mørk, "Microwave Photonic True Time Delay Based on Cross Gain Modulation in Semiconductor Optical Amplifiers," 15th OptoElectronics and Communications Conference (OECC2010) Technical Digest, July 2010, Sapporo Convention Center, Japan, pp. 202-203.

[32] Y. O. Barmenkov, J. L. Cruz, A. Díez, M. V. Andrés, "Electrically tunable photonic true-time-delay line," Optics Express, Vol. 18, No. 17, 16 August 2010, pp. 1785917864.

[33] Z. Ji, W. Zhu, Z. Li, Y. Sun, Q. Wang, “A Novel Method of Optical True Time Delay in Phased Array Antenna," Proceedings of the 9th European Radar Conference, 31 Oct - 2 Nov 2012, Amsterdam, The Netherlands, pp. 492-495.

[34] S. Garcia and I. Gasulla, "Design of heterogeneous multicore fibers as sampled truetime delay lines," Optics Letters, Vol. 40, No. 4, February 15, 2015, pp. 621-624.

[35] P. A. Costanzo Caso, L. A. Bulus Rossini, R. Duchowicz y E. Sicre, "Procesamiento Fotónico de Señales de RF y MO, y su Aplicación Para el Diseño de un Conformador de Haz Óptico", XIV Reunión de Trabajo en Procesamiento de la Información y Control (RPIC), Oro Verde, Entre Ríos, 16 al 18 de Noviembre de 2011.

[36] P. A. Costanzo Caso, S. Rabal, E. Paulucci, A. Giordana, y L. A. Bulus Rossini, "Practical impairments in FBG-based true time delays," Latin America Optics \& Photonics Conference (LAOP), Sao Sebastiao, Brasil, 2012, 1-3.

[37] S. Tedjini, and A. HO-QUOC LEMO-URA, "Theoretical \& Experimental Study of Single Mode Fiber Optical Ring Resonators for Microwave Applications," IEEE MTTS International Microwave Symposium Digest, 1995.

[38] K. Jinguji, "Synthesis of Coherent Two-Port Optical Delay-Line Circuit with Ring Waveguides," Journal of Lightwave Technology, Vol. 14, No. 8, August 1996, pp. 1882-1898.

[39] J. E. Heebner, V. Wong, A. Schweinsberg, R. W. Boyd, and D. J. Jackson, "Optical Transmission Characteristics of Fiber Ring Resonators," IEEE Journal of Quantum Electronics, Vol. 40, No. 6, June 2004, pp. 726-730.

[40] C.G.H. Roeloffzen, L. Zhuang, R.G. Heideman, A. Borreman, and W. van Etten, "Ring resonator-based Tunable Optical Delay Line in LPCVD Waveguide Technology," Proceedings Symposium IEEE/LEOS Benelux Chapter, 2005, Mons, pp. 79-82.

[41] L. Zhuang, C. G. H. Roelofzen, R. G. Heideman, A. Borreman, A. Meijerink, and W. van Eten, "Single -Chip Ring Resonator-Based 1x8 Optical Beam Forming Network in 
CMOS-Compatible Waveguide Techonology," IEEE Photonics Technology Letters, Vol. 19, No. 15, August 1 2007, pp. 1130-1132.

[42] H. Schippers, J. Verpoorte, P. Jorna, A. Hulzinga, A. Meijerink, C. G. H. Roeloffzen, L. Zhuang, D. A. I. Marpaung, W. van Etten, R. G. Heideman, A. Leinse, A. Borreman, and M. Hoekman, "Broadband Conformal Phased Array with Optical Beam Forming for Airborne Satellite Communication," IEEE Aerospace Conference, 2008.

[43] J. Cardenas, M. A.Foster,N. Sherwood-Droz, C. B. Poitras, H. L. R. Lira, B. Zhang, A. L. Gaeta, J. B. Khurgin, P. Morton, and M. Lipson, "Wide-bandwidth continuously tunable optical delay line using silicon microring resonators," Optics Express, Vol. 18, No. 25, December 2010, pp. 26525-26534. 


\section{Capítulo 2 - Resonador óptico en anillo}

\subsection{Introducción}

Los resonadores ópticos en anillo (Optical Ring Resonator (ORR)) han sido ampliamente investigados en los últimos años debido a sus atractivas características, que los hacen apropiados para una gran variedad de aplicaciones en el campo del procesamiento fotónico de señales, entre las que pueden mencionarse: la implementación de filtros sintonizables, sensores fotónicos, retardadores ópticos, resonadores láser, multiplexores add\&drop de canales multiplexados en longitud de onda (Wavelength Division Multiplexing (WDM)), compensadores de dispersión, y más.

En particular, una de las aplicaciones ampliamente difundidas últimamente consiste en la implementación de líneas de retardo para sistemas ópticos conformadores de haz (Optical Beamformer (OBF)) [1-3], con las ventajas que conlleva el procesamiento fotónico de señales de microondas (MO), como peso y tamaño reducidos del sistema, gran ancho de banda, atenuación baja y constante en un amplio rango de frecuencias, inmunidad al ruido electromagnético, baja dispersión, etc. Pero tal vez la más interesante de las ventajas frente a su contraparte electrónica sea la independencia de la frecuencia de los retardos temporales producidos, evitando el estrabismo del haz, o beam squint, que aparece cuando componentes de distinta frecuencia presentes en la señal son irradiadas con distinto ángulo. 


\subsection{Descripción general}

La configuración más simple de un ORR consiste en dos guías de onda, una con forma recta y otra con forma de camino cerrado o anillo, situadas muy próximas entre sí de manera que sus campos evanescentes se solapen. De esta manera, parte de la luz que se propaga por la guía recta se acopla al anillo, que en ciertas condiciones constituye una cavidad resonante óptica.

Un dispositivo modulador de fase (MF) dentro del anillo se utiliza para agregar una fase adicional a la onda que se propaga por el mismo con el fin de sintonizar el anillo en distintas condiciones de resonancia. Así, se obtiene un dispositivo de dos puertos (entrada y salida), como se muestra en la Fig. 2.1.

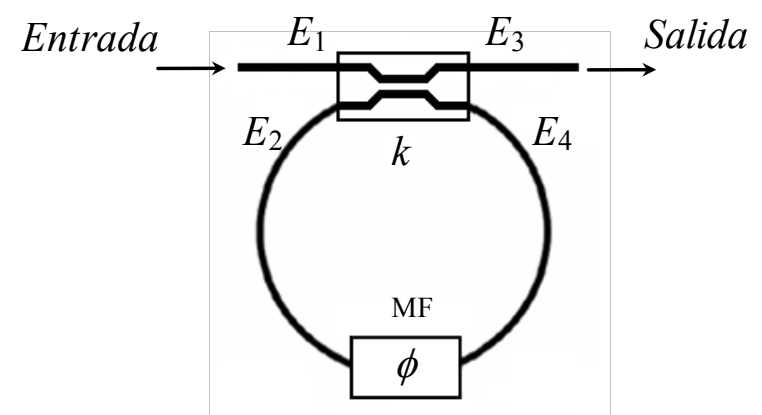

Figura 2.1: Esquema de un ORR controlable. Configuración básica.

Existen diversas formas de implementar un dispositivo como el mencionado: una forma, si se emplean componentes discretos, consiste en conectar los puertos de salida y de entrada (aquellos asociados al mismo núcleo) de un acoplador direccional mediante un tramo de fibra óptica, formando un anillo [4-6].

Por otro lado, con el avance de la tecnología y las técnicas de fabricación, hoy en día es posible la construcción de dispositivos integrados de dimensiones mucho menores y características mejoradas $[7,8]$. 


\subsection{Teoría de funcionamiento}

De la potencia óptica que ingresa al puerto de entrada, una fracción $k$ se acopla al anillo y se propaga por el mismo. Como se verá más adelante, el campo eléctrico que se acopla sufre un desplazamiento de fase de $90^{\circ}$, y esto es importante para el funcionamiento del dispositivo. La onda acoplada se propaga por el anillo, y el recorrido que realiza se conoce como round-trip. Para que el presente análisis sea válido, el tiempo de coherencia de la fuente de luz debe ser mayor al tiempo de round-trip.

Luego de cada round-trip, los sucesivos acoplamientos de luz hacia el anillo se suman y conforman una serie geométrica con razón menor a 1 que converge a una cierta condición de funcionamiento en estado estacionario. En este estado, los términos que se acoplaron y se encuentran en fase interfieren constructivamente, condición que se conoce como resonancia. Para que esto se cumpla es necesario que el desplazamiento de fase, $\theta_{r t}$, que experimenta la luz al propagarse un round-trip sea múltiplo de $2 \pi$.

Si $\lambda$ es la longitud de onda de la luz en el vacío, $n_{e f}$ el índice de refracción efectivo de la guía que constituye el anillo, y $L$ la longitud del mismo, la condición de resonancia se expresa como:

$$
\theta_{r t}=\frac{2 \pi L n_{e f}}{\lambda}=2 \pi N \quad \rightarrow \quad L=\frac{\lambda N}{n_{e f}}, \quad N \in \mathbb{Z}
$$

Como se observa en la Ec. (2.1), esto se cumple cuando $L$ es un múltiplo entero de la longitud de onda de la luz que se propaga por la guía de ondas.

Dentro del anillo, la intensidad de la luz que resuena está determinada por la fracción de potencia que se acopla al y desde el mismo, las pérdidas por propagación y el grado de interferencia entre las ondas. Si se considera que el factor de acoplamiento y las pérdidas son independientes de la longitud de onda, la intensidad de la luz es menor a medida que se aparta de la condición de resonancia.

Cuando todos los términos que se suman están en fase, la interferencia es constructiva y la intensidad de la onda en estado estacionario es máxima. Las intensidades de aquellas ondas que no cumplen con la condición de resonancia resultan menores.

Por otro lado, parte de la luz que resuena en el anillo también se acopla nuevamente a la guía recta en las sucesivas vueltas, pero lo hace con un desfasaje de $180^{\circ}$ debido al 
desfasaje de $90^{\circ}$ que se introduce, como se mencionó, cada vez que se acopla. Esta luz interfiere destructivamente con la fracción $1-k$ que no se acopló al anillo. El grado de atenuación de la luz que se transmite a la salida depende de las amplitudes de las ondas que interfieren y por lo tanto de la intensidad de la luz que resuena dentro del anillo. A la frecuencia de resonancia, esta intensidad es máxima, y por lo tanto a la salida del dispositivo resulta mínima, funcionando de esta manera como un filtro notch.

Para cierta combinación de valores de factor de acoplamiento y pérdidas, la atenuación a la frecuencia de resonancia es máxima y no se transmite luz a la salida. En esta condición, que será analizada más adelante, se dice que el acoplamiento es crítico.

\subsection{Modelo matemático}

\section{Acoplador direccional}

Es el componente principal del dispositivo ya que permite acoplar la luz entre las guías de onda. Es un dispositivo de $2 \times 2$ puertos (dos de entrada y dos de salida) mediante el cual se divide la potencia óptica incidente en uno de los puertos de entrada, enviando cada fracción a un puerto de salida.

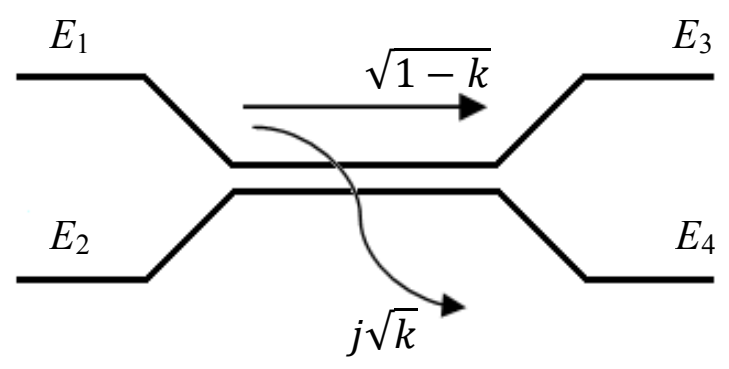

Figura 2.2: Esquema de un acoplador $2 \times 2$.

En la Fig. 2.2 se muestra el esquema de un acoplador $2 \times 2$, indicando el campo eléctrico $E_{j}(j=1 ; 2 ; 3 ; 4)$, en cada uno de sus cuatro puertos. La matriz de transferencia del mismo se demuestra en el Apéndice A y está dada por la siguiente expresión:

$$
T_{c}=\left[\begin{array}{l}
E_{3} \\
E_{4}
\end{array}\right]=\xi \cdot\left[\begin{array}{cc}
\sqrt{1-k} & j \sqrt{k} \\
j \sqrt{k} & \sqrt{1-k}
\end{array}\right] \cdot\left[\begin{array}{c}
E_{1} \\
E_{2}
\end{array}\right]
$$


En la Ec. (2.2), $k$ es la fracción de la potencia de entrada que se acopla de una guía a la otra, y $\xi \leq 1$ es un factor que representa las pérdidas en exceso $P_{E}$ que existen en un acoplador real, y se definen como

$$
P_{E}[d B]=10 \cdot \log _{10}\left(\frac{P_{1}}{P_{3}+P_{4}}\right)=20 \cdot \log _{10}(\xi)
$$

donde $P_{j}(j=1 ; 2 ; 3 ; 4)$ son las potencias en cada uno de los puertos. Valores típicos para estas pérdidas son del orden de $0,2 \mathrm{~dB}$ o menores, en acopladores de fibra óptica.

Como puede notarse a partir de la Ec. (2.2), cuando un campo se acopla de un núcleo al otro, lo hace con un desplazamiento de fase de $\pi / 2$.

\section{Guía de ondas}

El anillo o camino cerrado que conforma la cavidad resonante se implementa mediante una guía de ondas óptica (puede ser integrada o un tramo de fibra óptica monomodo) que se encuentra caracterizada por su longitud $L$, coeficiente de atenuación de intensidad $\alpha$, e índice de refracción efectivo $n_{e f}$. Haciendo referencia a la Fig. 2.1, si $E_{4}$ y $E_{2}$ son las amplitudes complejas de los campos eléctricos a la entrada y a la salida de la guía respectivamente, puede escribirse:

$$
\begin{aligned}
E_{2}= & E_{4} \cdot \exp \left(-\frac{\alpha L}{2}\right) \cdot \exp (-j \phi) \exp [-j \Omega(\omega)] \\
& =E_{4} \cdot \sqrt{a} \cdot \exp (-j \phi) \cdot \exp [-j \Omega(\omega)]
\end{aligned}
$$

Donde $a=\exp (-\alpha L) \leq 1$ representa la atenuación de intensidad e incluye tanto las pérdidas por absorción del material como las pérdidas del MF y otras fuentes de atenuación, y aunque depende de la frecuencia, en el presente trabajo se considera constante en el rango de interés, mientras que $\Omega(\omega)$ es el desplazamiento de fase que se produce al propagarse la onda una distancia $L$.

$$
\Omega(\omega)=\beta \cdot L=\frac{2 \pi n_{e f}}{\lambda} \cdot L=2 \pi f \cdot \frac{n_{e f}}{c} \cdot L=\omega T_{R T}
$$

En (2.5), $T_{R T}=n_{e f} L / c$ es el tiempo de round-trip mencionado anteriormente, y es el tiempo que tarda la onda en recorrer una vuelta por el anillo.

Se define el Free Spectral Range (FSR) o rango espectral libre, como la distancia en frecuencia entre dos picos de resonancia sucesivos, siendo $\mathrm{FSR}=1 / T_{R T}$. Por esta razón, 
suele referirse a $\Omega(\omega)=\omega T_{R T}$ como la frecuencia angular normalizada con respecto al FSR.

\section{Función de transferencia del resonador óptico en anillo}

La cavidad resonante se forma al conectar un puerto de entrada y uno de salida del acoplador mediante la guía con forma de anillo, como se muestra en la Fig. 2.1, y su función de transferencia (Ec. (2.6)) se obtiene al combinar (2.2) y (2.4). Luego de algunas operaciones algebraicas, mediante las que se resuelve la serie geométrica mencionada en la descripción del funcionamiento de la cavidad, se obtiene:

$$
t_{31}(\Omega)=\frac{E_{3}}{E_{1}}=\left[\frac{\xi \sqrt{1-k}-\xi^{2} \sqrt{a} \cdot e^{-j \phi} e^{-j \Omega}}{\left(1-\xi \sqrt{1-k} \cdot \sqrt{a} \cdot e^{-j \phi} e^{-j \Omega}\right)}\right]
$$

La Ec. (2.6) describe el comportamiento del resonador en estado estacionario. El coeficiente de transmisión de intensidad $T_{31}$ se define como el módulo de la función de transferencia elevado al cuadrado (Ec. (2.7)), mientras que el argumento de la misma determina la fase (Ec. (2.8)).

El retardo de grupo normalizado con respecto a $T_{R T}$ se define como la derivada cambiada de signo de (2.8) con respecto a la frecuencia normalizada $\Omega(\tau=-d \theta / d \Omega)$, luego el retardo de grupo absoluto, medido en unidades de tiempo, es $\tau_{\mathrm{g}}=\tau \cdot T_{R T}$.

$$
\begin{gathered}
T_{31}(\Omega)=\left|t_{31}(\Omega)\right|^{2}=\frac{\xi^{4} a+\xi^{2}(1-k)-2 \xi^{3} \sqrt{a} \cdot \sqrt{1-k} \cdot \cos (\phi+\Omega)}{1+\xi^{2}(1-k) a-2 \xi \sqrt{1-k} \cdot \sqrt{a} \cdot \cos (\phi+\Omega)} \\
\theta_{31}(\Omega)=\tan ^{-1}\left(\frac{\xi^{2} \sqrt{a} \cdot \operatorname{sen}(\phi+\Omega)}{\xi \sqrt{1-k}-\xi^{2} \sqrt{a} \cdot \cos (\phi+\Omega)}\right)-\tan ^{-1}\left(\frac{\xi \sqrt{1-k} \cdot \sqrt{a} \cdot \operatorname{sen}(\phi+\Omega)}{1-\xi \sqrt{1-k} \cdot \sqrt{a} \cdot \cos (\phi+\Omega)}\right) \\
\tau_{31}(\Omega)=\frac{\xi^{4} a-\xi^{3} \sqrt{a} \cdot \sqrt{1-k} \cdot \cos (\phi+\Omega)}{\xi^{2}(1-k)-2 \xi^{3} \sqrt{1-k} \cdot \sqrt{a} \cdot \cos (\phi+\Omega)+\xi^{4} a}+\frac{\xi \sqrt{1-k} \cdot \sqrt{a} \cdot \cos (\phi+\Omega)-\xi^{2}(1-k) a}{1-2 \xi \sqrt{1-k} \cdot \sqrt{a} \cdot \cos (\phi+\Omega)+\xi^{2}(1-k) a}
\end{gathered}
$$

En la siguiente sección se muestran cualitativamente el coeficiente de transmisión de intensidad, la fase y el retardo de grupo normalizado en función de $\Omega$. 


\subsection{Simulaciones}

A partir de la Ec. (2.6), se realizaron simulaciones numéricas en Matlab ${ }^{\circledR}$ que permitieron obtener la respuesta del dispositivo en función de la frecuencia angular normalizada para una dada condición de acoplamiento y fase adicional. A continuación se presentan gráficamente los resultados.

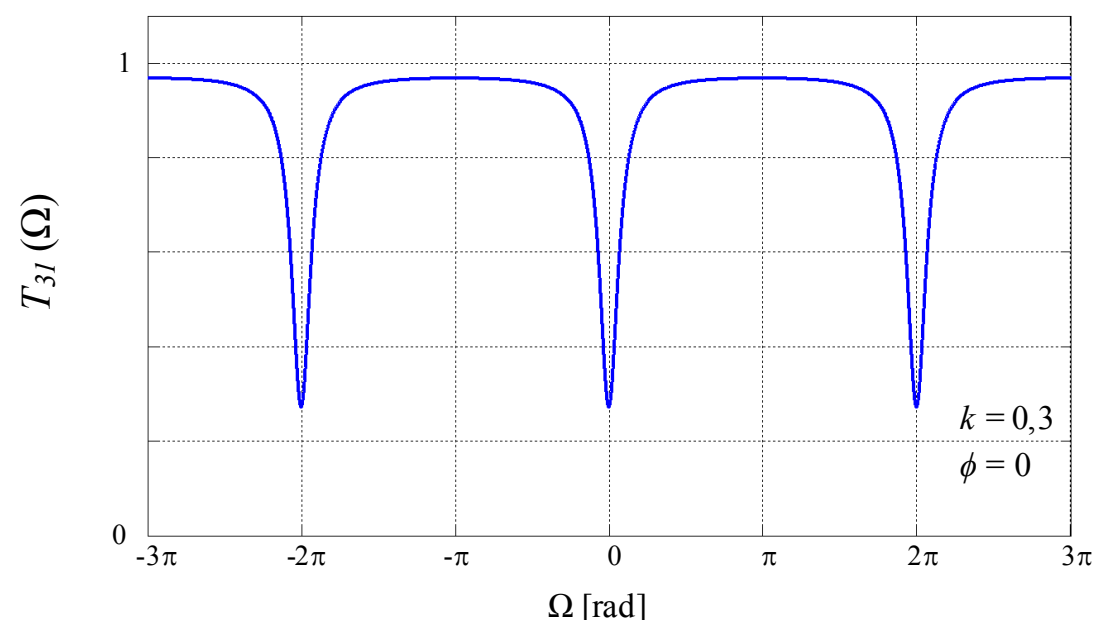

Figura 2.3: Coeficiente de transmisión de intensidad (Ec. (2.7))

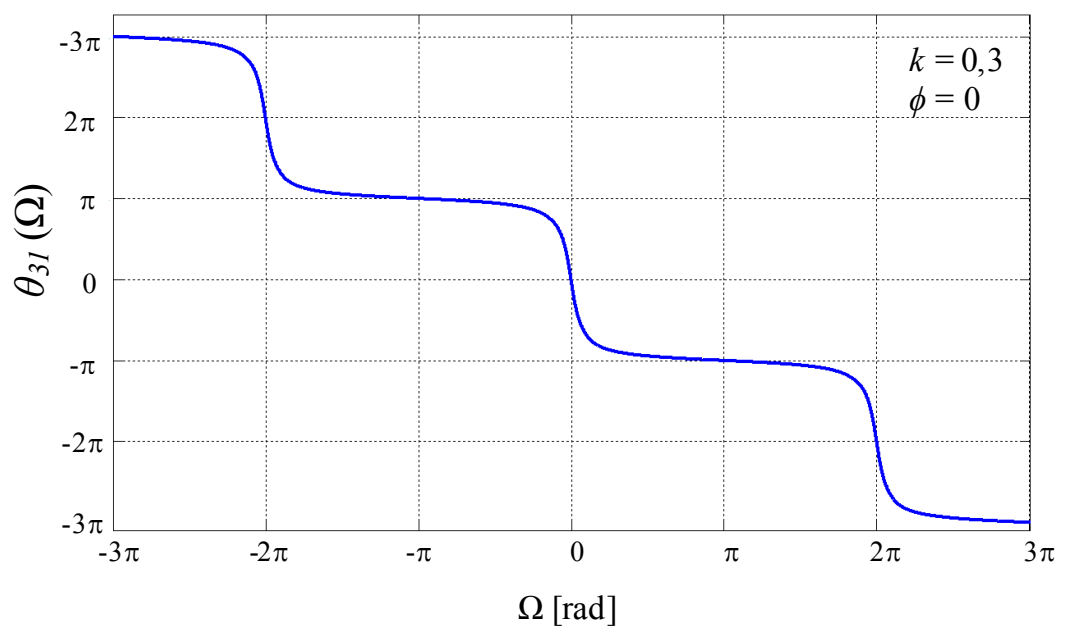

Figura 2.4: Fase de la transferencia (Ec. (2.8)). 


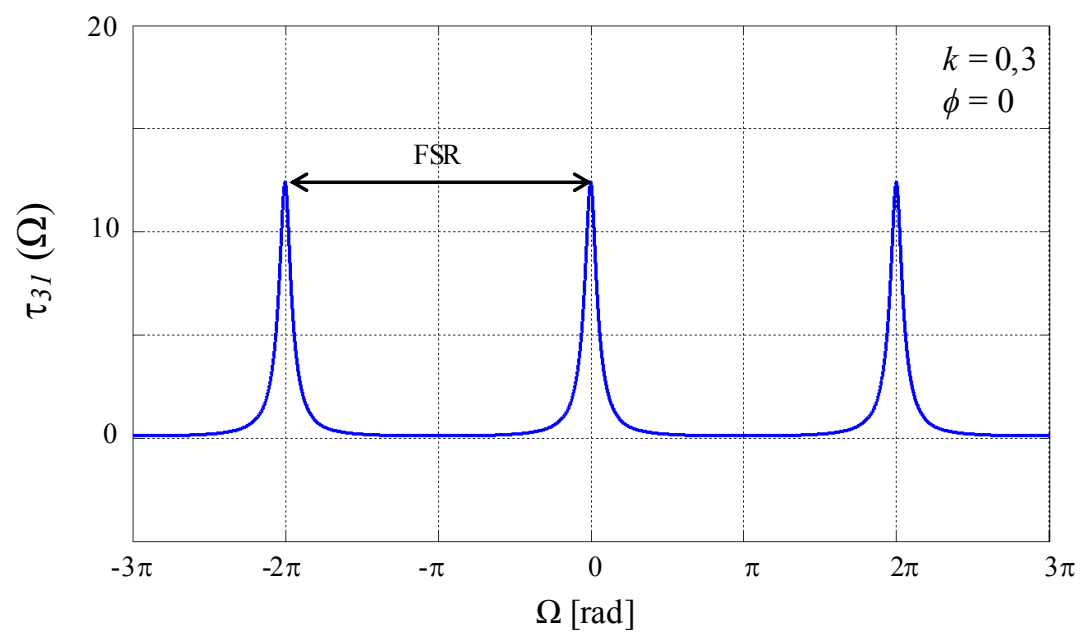

Figura 2.5: Retardo de grupo normalizado (Ec. (2.9))

Como puede verse en la Fig. 2.3, el dispositivo se comporta como un filtro notch que atenúa más las frecuencias cercanas a la de resonancia, mientras que aquellas frecuencias más alejadas se transmiten con muy baja atenuación. Además, cabe destacar la periodicidad de la respuesta, que se repite cada $2 \pi$ radianes de la frecuencia angular normalizada.

La fase (Fig. 2.4) presenta una forma escalonada, con saltos de $2 \pi$ radianes y con pendiente abrupta en un entorno de cada resonancia. Esa es la razón por la que el retardo de grupo también presenta picos periódicos, como se observa en la Fig. 2.5, donde se indica, además, el rango espectral libre (FSR), es decir la distancia entre dos picos de resonancia sucesivos. En este caso, el FSR es de $2 \pi$, pero al desnormalizarlo se obtiene su valor en $\mathrm{Hz}$. Por ejemplo, para los valores utilizados en estas simulaciones, corresponde un FSR de alrededor de 5,38 GHz.

La característica más interesante que se observa en la Fig. 2.5 es que el retardo de grupo normalizado toma valores mayores a 1. Esto significa que para ciertas frecuencias, el retardo de grupo desnormalizado es mayor que el tiempo que tarda la luz en recorrer el anillo (tiempo de round-trip). Como se verá más adelante, a través de los parámetros de control (particularmente, el factor de acoplamiento) puede modificarse el valor pico del retardo a la frecuencia de resonancia y así implementar retardos variables en forma cuasi continua.

La Fig. 2.6 muestra un solo período de la respuesta de un ORR con el fin de indicar algunas características con mayor claridad. 

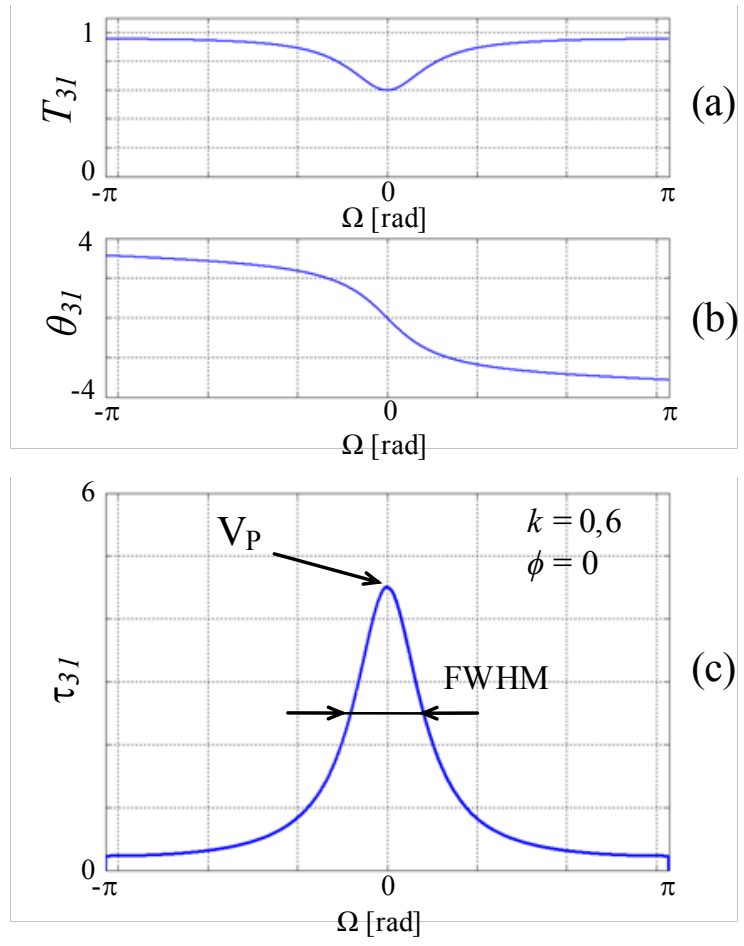

Figura 2.6: Respuesta de un ORR: (a) Coeficiente de transmisión de intensidad;

(b) Fase y (c) Retardo de grupo normalizado.

Cabe aclarar que en este caso, las condiciones de acoplamiento no son las mismas que en las figuras anteriores, ya que el desplazamiento de fase adicional sigue siendo $0 \mathrm{rad}$, pero el valor del factor de acoplamiento es 0,6 .

El retardo de grupo alcanza su pico máximo, $\mathrm{V}_{\mathrm{P}}$, a la frecuencia de resonancia y tiene un ancho a mitad del máximo (Full Width at Half Maximum) indicado como FWHM. En el Apéndice B se describe un algoritmo para calcular estos dos parámetros de la respuesta a partir de la Ec. (2.9). Cabe aclarar que la forma de campana que exhibe la respuesta, aunque pudiera parecer, no es gaussiana.

\section{Pa rá metros de control}

Una vez construido, un ORR posee dos parámetros de control a los cuales se tiene acceso para modificar las características de la respuesta. Estos parámetros de control son: el factor de acoplamiento, $k$, y el desplazamiento de fase adicional, $\phi$. A continuación se muestra la influencia que éstos tienen en el retardo de grupo.

En la Fig. 2.7(a) se presentan tres casos de acoplamiento: 0,5; 0,7 y 1. En todos los casos, la fase adicional se mantuvo en cero y por lo tanto la frecuencia de resonancia no se 
vio modificada. Cabe hacer notar que para el caso en que $k=1$ el retardo de grupo normalizado es constante para todas las frecuencias y de valor unitario. Esto significa que toda la luz que ingresa al puerto de entrada se acopla al anillo y luego de un tiempo de round-trip vuelve a acoplarse totalmente a la guía recta en el puerto de salida.

En la Fig. 2.7(b), por el contrario, la fase adicional tomó los valores $0, \pi / 2$ y - $\pi / 2$ mientras que el factor de acoplamiento se mantuvo igual a 0,6 . En este caso, el valor pico no se modificó, pero la respuesta se desplazó en frecuencia manteniendo su forma original, como podía intuirse al analizar con detalle la Ec. (2.6).

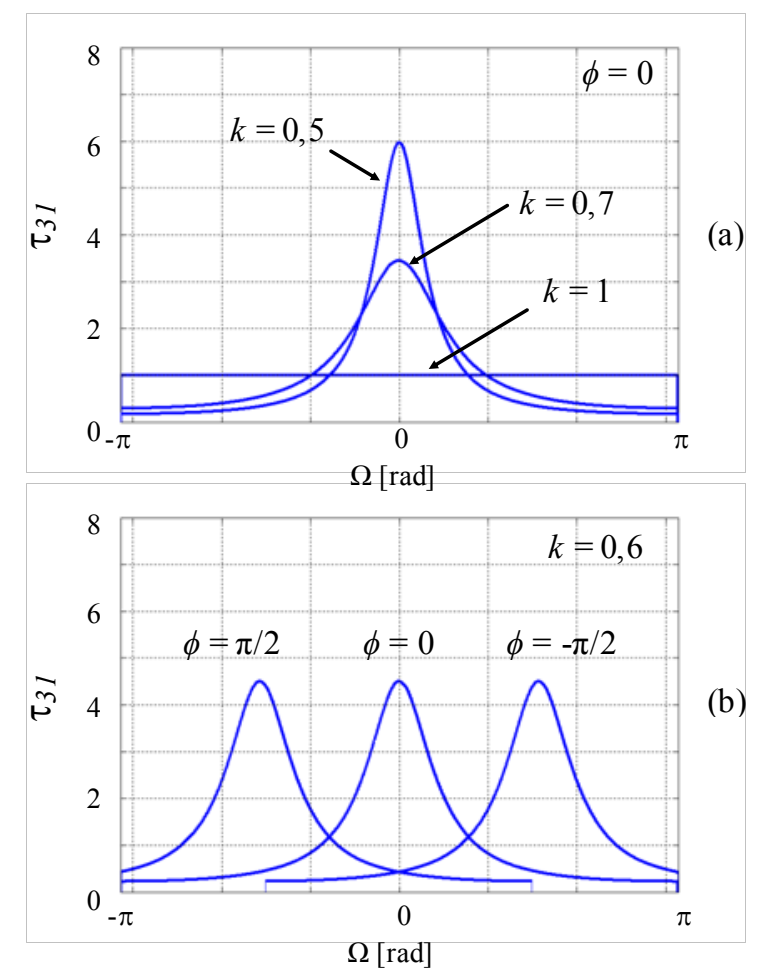

Figura 2.7: Retardo de grupo normalizado: (a) Parámetro $k$; (b) Parámetro $\phi$.

\section{Implementación de los parámetros de control}

Los dos parámetros que controlan la respuesta de un ORR pueden implementarse aprovechando el efecto termoóptico, que consiste el cambio del índice de refracción en una sección de la guía debido a un cambio local de temperatura. El cambio de índice produce, a su vez, un desplazamiento de fase en la luz que se propaga por la guía. 
Con este fin se utilizan calentadores ubicados sobre la guía óptica que consisten en piezas de metal (pueden ser de oro o cromo) de dimensiones determinadas para lograr cierto valor de resistencia eléctrica que puede estar en el orden de los $800 \Omega$. Cuando se aplica una diferencia de tensión a los extremos de uno de estos calentadores, circula una corriente por él y la potencia eléctrica se disipa en forma de calor. Se puede demostrar que el desplazamiento de fase que se produce en la luz que se propaga por la guía varía linealmente con la potencia disipada [9].

De esta forma, el desplazamiento de fase, $\phi$, que se utiliza para sintonizar la respuesta del dispositivo se logra colocando uno de estos calentadores en una sección de la guía que forma el anillo.

Por otro lado, para implementar un acoplador óptico $2 \times 2$ variable se puede emplear un interferómetro Mach-Zehnder simétrico, como se muestra en la Fig. 2.8. El esquema que se muestra dentro del recuadro en línea punteada, representa la implementación del acoplador variable mostrado en la Fig. 2.1.

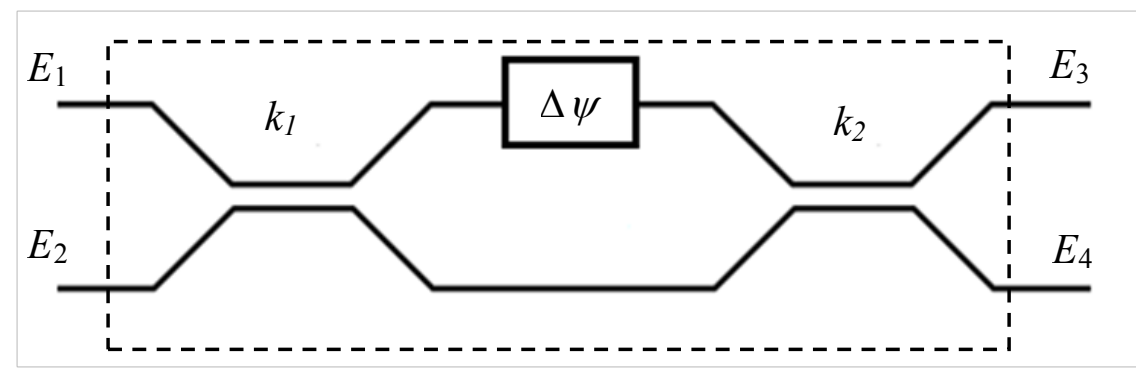

Figura 2.8: Acoplador variable a partir de dos acopladores fijos y un desplazamiento de fase.

Si se utilizan dos acopladores direccionales fijos, con valores de acoplamiento $k_{1} \mathrm{y} k_{2}, \mathrm{y}$ un desplazamiento de fase, $\Delta \psi$, en una de las ramas del interferómetro, las cuales son de igual longitud, puede demostrarse que la matriz de transferencia del conjunto tiene la misma forma que la Ec. (2.2). El factor de acoplamiento resultante, $k$, depende de los factores de acoplamiento de los acopladores que lo componen y del desplazamiento de fase, $\Delta \psi$. Si $k_{1}=k_{2}$, mediante el desplazamiento de fase adecuado pueden obtenerse, en teoría, valores de $k$ entre 0 y 1 . 


\subsection{Conclusiones}

Se presentó un dispositivo que posee múltiples aplicaciones dentro del campo del procesamiento fotónico de señales, se realizó una descripción del mismo, sus componentes principales y teoría de funcionamiento.

Se obtuvo un modelo matemático con el cual se realizaron simulaciones numéricas, analizando la respuesta del dispositivo en estudio. Se definieron e indicaron algunas características importantes de la respuesta, como el rango espectral libre y el ancho a mitad de altura del máximo.

Se consideró la variación de dos parámetros que permiten controlar la respuesta del dispositivo, como el factor de acoplamiento entre las guías y el desplazamiento de fase adicional dentro del anillo.

\section{Referencias}

[1] A. Meijerink, C. G. H. Roeloffzen, L. Zhuang, D. A. I. Marpaung, R. G. Heideman, A. Borreman, W. van Etten, "Phased array antenna steering using a ring resonator-based optical beam forming network," Proc. 13th IEEE/CVT Symp. Benelux, Liège, Belgium, 23 Nov. 2006, pp. 7-12.

[2] L. Zhuang, C. G. H. Roeloffzen, R. G. Heideman, A. Borreman, A. Meijerink, and W.van Etten, "Single-Chip Ring Resonator-Based 1 x 8 Optical Beam Forming Network in CMOS- Compatible Waveguide Technology," IEEE Photonics Technology Letters, Vol. 19, No. 15, August 1, 2007.

[3] J. Verpoorte, H. Schippers, P. Jorna, C.G.H. Roeloffzen1, D.A.I. Marpaung, R. Baggen and B. Sanadgo, "Architectures for $\mathrm{Ku}$-band broadband airborne satellite communication antennas," Nationaal Lucht- en Ruimtevaartlaboratorium, National Aerospace Laboratory NLR, NLR-TP-2010-537.

[4] J. E. Heebner, V. Wong, A. Schweinsberg, R. W. Boyd, and D. J. Jackson, "Optical Transmission Characteristics of Fiber Ring Resonators," IEEE Journal Of Quantum Electronics, Vol. 40, No. 6, pp. 726-730, June 2004.

[5] S. Tedjini, "Theoretical \& Experimental Study of Single Mode Fiber Optical Ring Resonators for Microwave Applications," IEEE MIT -S Digest, pp. 1491-1494, 1995.

[6] G. S. Pandian, F. E. Seraji, "Optical pulse response of a fibre ring resonator," IEE Proceedings-J, Vol. 138, No. 3, pp. 235-239, June 1991.

[7] C.G.H. Roeloffzen, L. Zhuang, R.G. Heideman, A. Borreman, and W. van Etten, "Ring resonator-based Tunable Optical Delay Line in LPCVD Waveguide Technology," Proceedings Symposium IEEE/LEOS Benelux Chapter, 2005, Mons, pp 79-82. 
[8] A. Melloni, R. Costa, P. Monguzzi and M. Martinelli, "Ring-resonator filters in silicon oxynitride technology for dense wavelength-division multiplexing systems," Optics Letters, Vol. 28, No. 17, September 1, 2003.

[9] Chris Roeloffzen, Passband flattened binary-tree structured add-drop multiplexers using SiON waveguide technology, $\mathrm{PhD}$ thesis (ISBN 90-365-1803-2), University of Twente, Enschede, The Netherlands, 2002. 
Heinrich Sebastian Rabal - Líneas de Retardo Real Para Conformación Óptica de Haces de Microondas 


\section{Capítulo 3 - Caracterización de la respuesta de un ORR}

\subsection{Introducción}

En este capítulo se estudia la respuesta del resonador óptico en anillo (ORR) presentado en el Capítulo 2, analizando la influencia de ciertos parámetros constructivos y de control en la respuesta del dispositivo. Las simulaciones que se presentan a continuación fueron realizadas en Matlab ${ }^{\circledR}$ a partir de la función de transferencia obtenida anteriormente, descripta por la Ec. (3.1).

$$
t_{31}(\Omega)=\frac{E_{3}}{E_{1}}=\left[\frac{\xi \sqrt{1-k}-\xi^{2} \sqrt{a} \cdot e^{-j \phi} e^{-j \Omega}}{\left(1-\xi \sqrt{1-k} \cdot \sqrt{a} \cdot e^{-j \phi} e^{-j \Omega}\right)}\right]
$$

Conocer la influencia de cada uno de los parámetros que intervienen en (3.1) es necesario para determinar la relevancia que podrían tener algunas desviaciones inevitables que se producen durante el proceso de fabricación, o debidas a cambios en las condiciones de trabajo, como puede ser el caso de la temperatura. Por otro lado, por medio de las simulaciones puede verificarse la gobernabilidad de la respuesta a través de los parámetros $k$ y $\phi$, que permiten modificar, dentro de ciertos rangos, las características de los retardos producidos.

\subsection{Simulaciones}

Utilizando el software Matlab ${ }^{\circledR}$, se realizaron simulaciones numéricas de la función de transferencia dada por la Ec. (3.1) para luego obtener el coeficiente de transmisión de intensidad, $T_{31}$, y el retardo de grupo normalizado, $\tau_{31}$, en función de la frecuencia angular 
normalizada, $\Omega$, definida en la Ec. (2.5). Inicialmente, se adoptaron los siguientes valores por defecto para los parámetros de la función de transferencia:

- Pérdidas por exceso del acoplador:

- Coeficiente de atenuación de la guía:

- Factor de acoplamiento:

- Radio del anillo:

- Índice efectivo de la guía:

- Coeficiente de modulación de fase:

$$
\begin{aligned}
& P_{E}=0,1 \mathrm{~dB}(\xi \cong 0,9886) \\
& \alpha=10 \mathrm{~dB} / \mathrm{m} \\
& k=0,5 \\
& r=0,006 \mathrm{~m} \\
& n_{\mathrm{ef}}=1,48 \\
& \phi=0 \mathrm{rad}
\end{aligned}
$$

Luego, en cada caso, se consideró la variación de sólo uno de ellos para mostrar su influencia en la respuesta del dispositivo. A continuación se presentan los resultados obtenidos.

\section{Factor de acoplamiento}

El factor de acoplamiento, $k$, representa la fracción de potencia que se acopla al anillo a través de la onda evanescente (Ver Apéndice A). La Fig. 3.1 muestra cómo se modifica la respuesta del resonador para una variación del mismo entre los valores 0,$8 ; 0,6 ; 0,4 ; 0.1$ y 0,05 .
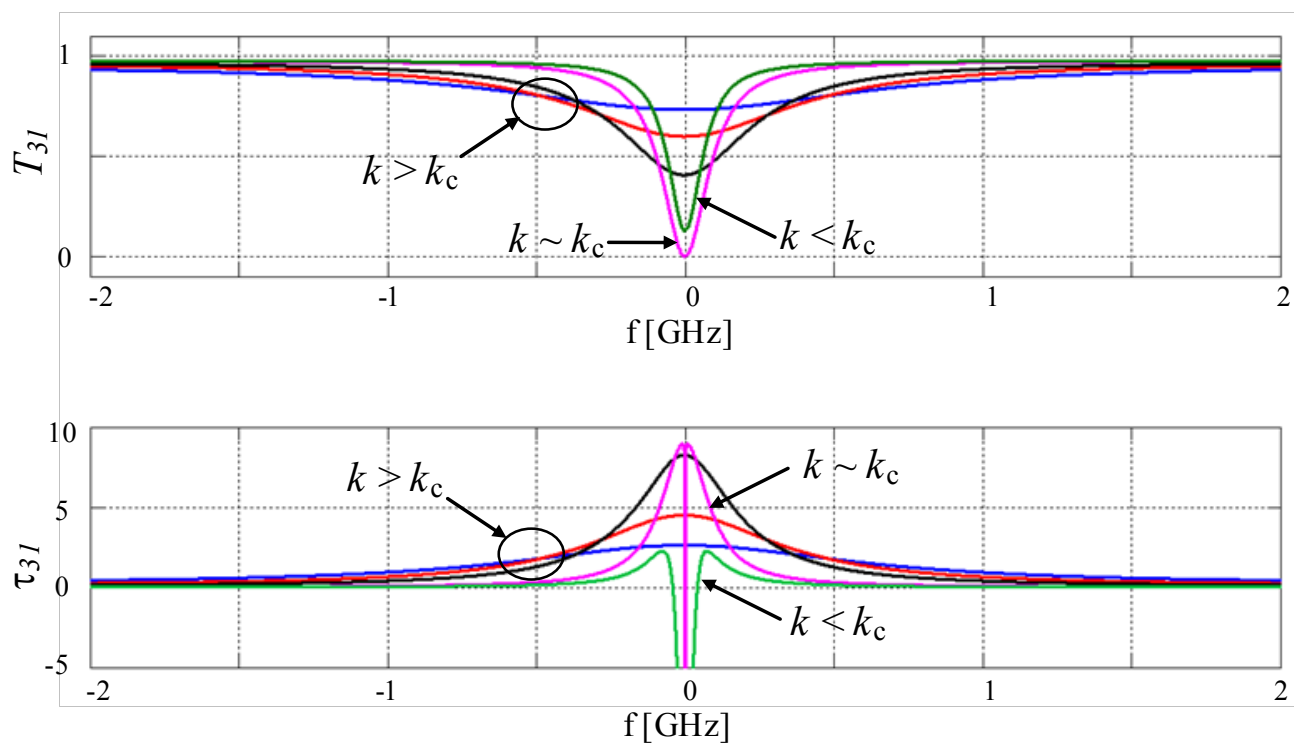

Figura 3.1: Variación del factor de acoplamiento: (a) Coeficiente de transmisión de intensidad; (b) Retardo de grupo normalizado. 
Como se puede ver en la Fig. 3.1(a), a medida que se reduce el factor de acoplamiento aumenta la atenuación a la frecuencia de resonancia y disminuye el ancho de la respuesta, es decir, se hace más selectiva. Cuando $k$ toma un cierto valor que denominaremos $k_{\mathrm{c}}$ (acoplamiento crítico), la transmisión de intensidad a la salida es cero, y al seguir reduciendo $k$ por debajo de dicho valor vuelve a aumentar, pero con una respuesta más angosta. Esto nos permite diferenciar dos zonas de trabajo: una sobreacoplada, con valores mayores a $k_{\mathrm{c}}$, y otra subacoplada, con valores menores a $k_{\mathrm{c}}$.

Aunque no se muestre en la figura, las variaciones del factor de acoplamiento, tanto en la zona sobreacoplada como en la subacoplada, no modifican la periodicidad de la respuesta (Figs. 2.3, 2.4 y 2.5), manteniendo el FSR invariable.

Por otro lado, en la Fig. 3.1(b) se observa que el pico del retardo de grupo aumenta al reducir el factor de acoplamiento hasta alcanzar su valor máximo en la condición crítica. En este punto, para frecuencias cercanas a la de resonancia aparecen retardos negativos que pueden entenderse si se recuerda que no son retardos absolutos, sino relativos a la señal portadora. Dentro de la zona sobreacoplada, se observa que el FWHM del retardo se reduce a medida que aumenta el valor pico, reduciendo el ancho de banda. Por otro lado, en la zona subacoplada no es posible hablar de un ancho a mitad de altura debido a la forma anómala del retardo. La condición de acoplamiento crítico se analizará con más detalle en el Capítulo 4.

\section{Coeficiente de atenuación de la guía de ondas}

Los valores de pérdidas, $\alpha$, se eligieron cercanos $10 \mathrm{~dB} / \mathrm{m}$ (valor obtenible en guías de onda integradas) pero con variación suficientemente grande $( \pm 20 \%)$ como para que los efectos sean notorios. En particular, se simuló la respuesta del ORR con pérdidas de: 8; 9; $10 ; 11$ y $12 \mathrm{~dB} / \mathrm{m}$. En la siguiente figura se muestran los resultados obtenidos. 

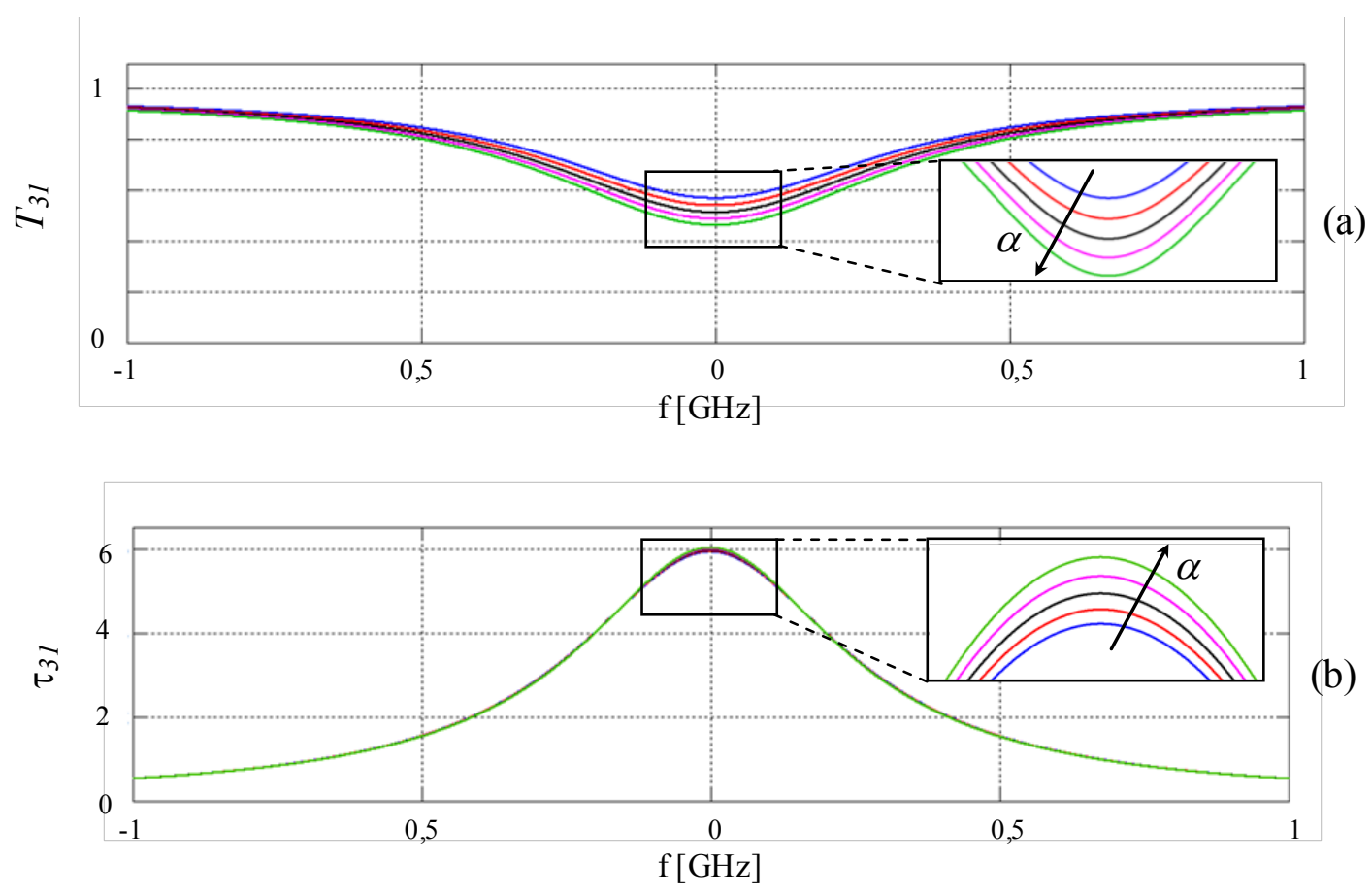

Figura 3.2: Variación de las pérdidas de la guía: (a) Coeficiente de transmisión de intensidad; (b) Retardo de grupo normalizado.

Debido a que el FSR no se vio modificado, la Fig. 3.2 sólo muestra un período de la respuesta. En particular, en la Fig. 3.2(a) se muestra el coeficiente de transmisión de intensidad y se advierte que un aumento de las pérdidas produce un pequeño aumento en la atenuación, sobre todo a la frecuencia de resonancia. En un recuadro dentro de la misma figura se muestra un acercamiento que permite apreciar con más detalle el incremento de la atenuación. Con una flecha se indica el sentido de crecimiento de $\alpha$. La variación del coeficiente de transmisión de intensidad con respecto a su valor nominal (para $\alpha=10 \mathrm{~dB} / \mathrm{m}$ ) se mantuvo menor a $\pm 10,9 \%$.

Por otro lado, en la Fig. 3.2(b) se observa el retardo de grupo normalizado. Nuevamente, las variaciones son tan pequeñas que para apreciarlas correctamente se recurre a un acercamiento. Puede verse que el valor pico crece ligeramente a medida que aumentan las pérdidas. En particular, para el rango de valores de pérdidas simulado, el valor pico del retardo sufrió una variación menor a $\pm 0,9 \%$ con respecto a su valor no minal. 


\section{Radio del anillo}

El aumento del radio del anillo donde resuena la luz se traduce en una mayor longitud del mismo, y por lo tanto en mayores tiempos de round-trip, lo que implica, a priori, retardos de mayor valor. En la Fig. 3.3(a) y (b) se observan el coeficiente de transmisión de intensidad y el retardo de grupo normalizado que se obtienen para distintos radios del anillo: $4 ; 5 ; 6 ; 7 ;$ y $8 \mathrm{~mm}$.
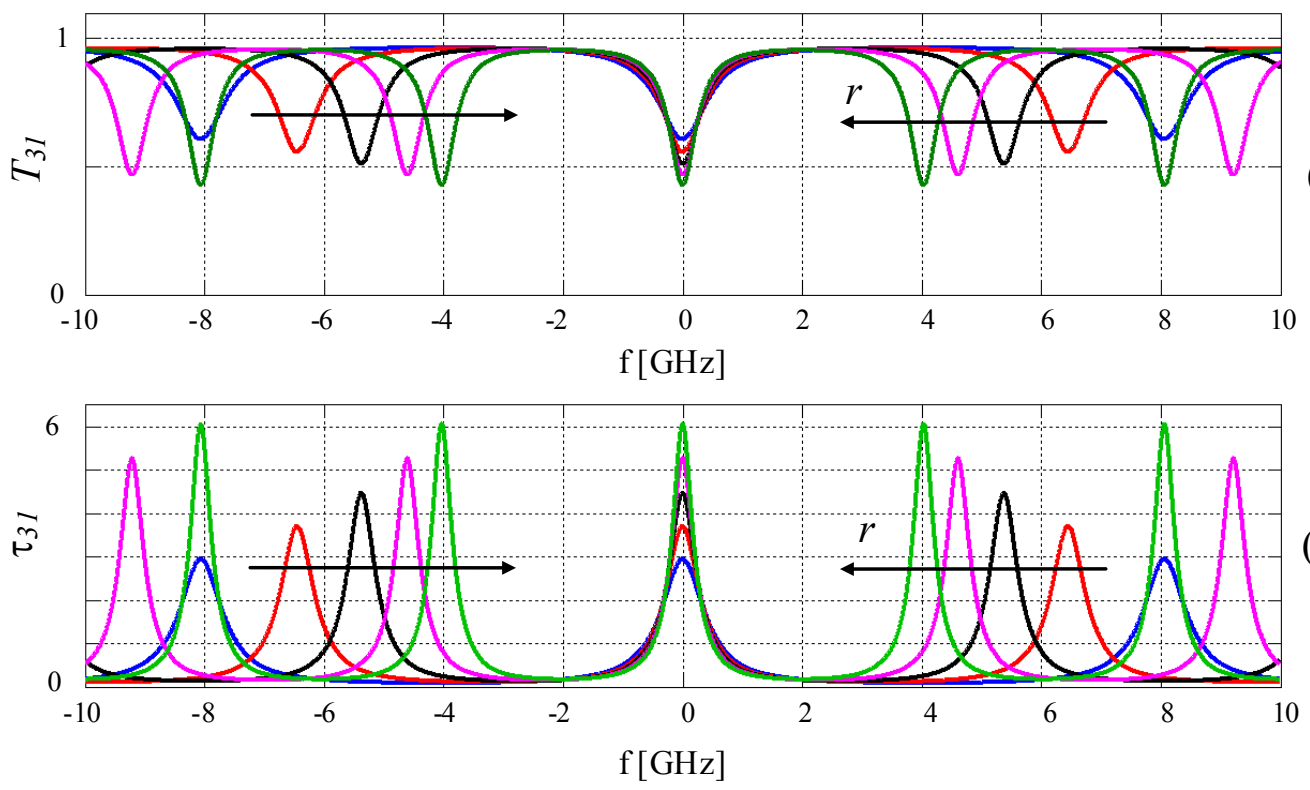

Figura 3.3: Variación del radio del anillo: (a) Coeficiente de transmisión de intensidad; (b) Retardo de grupo normalizado.

Al aumentar las dimensiones del anillo manteniendo la velocidad de la luz (es decir, sin modificar el índice $n_{\mathrm{ef}}$ ), el $\mathrm{T}_{\mathrm{RT}}$ se hace mayor y por lo tanto se reduce la distancia entre los picos tanto de atenuación como de retardo. Además, a mayor longitud del anillo también corresponden más pérdidas acumuladas en cada round-trip, y esto se evidencia en los cambios de amplitud de los picos de atenuación en la Fig. 3.3(a), que alcanzan una variación cercana $\mathrm{a} \pm 18,8 \%$.

En la Fig. 3.3(b), las curvas de retardo se encuentran normalizadas con respecto al mayor tiempo de round-trip dentro del rango de variación de $r$ para que puedan apreciarse las diferencias entre los valores pico. 
Se observa una gran variación (cercana $\mathrm{a} \pm 35,4 \%$ ) y, como era de esperar, con anillos de radios mayores se obtienen retardos con mayor valor pico. Además, como elárea bajo la curva del retardo debe mantenerse constante, al aumentar el valor pico también se reduce el FWHM, haciendo a los retardos más selectivos. Esta relación de compromiso entre el valor pico y el ancho de banda implica que se si desean implementar líneas de retardo para señales de banda ancha, los dispositivos deben construirse del menor tamaño posible, y para ello es conveniente investigar distintas técnicas de fabricación de óptica integrada [1-4].

\section{Pérdidas del acopla dor}

Las pérdidas en el acoplador aparecen debido a que no toda la potencia de entrada se transfiere a la salida del mismo. Este tipo de pérdidas depende del tipo de dispositivo, el material y método de construcción. Debido a los valores relativamente bajos que suelen tomar, en muchos trabajos anteriores no son tenidas en cuenta. Sin embargo aquí se analizará su influencia en la respuesta del resonador para mostrar que dicha simplificación está justificada.

Para implementar la variación de las pérdidas del acoplador, $P_{E}$, se utilizó la Ec. (3.2), que las relaciona con el factor $\xi$ :

$$
P_{E}[d B]=20 \cdot \log _{10}(\xi)
$$

En la Fig. 3.4(a) y (b) pueden observarse el coeficiente de transmisión de intensidad y el retardo de grupo normalizado cuando las pérdidas del acoplador toman los valores: 0,08; 0,$09 ; 0,1 ; 0,11$ y $0,12 \mathrm{~dB}$. En 3.4(a), un aumento en las pérdidas produce un aumento en la atenuación, no sólo de la longitud de onda que resuena, sino de todo el espectro, como cabe esperar, ya que estas pérdidas afectan por igual a todos los campos que interactúan en las entradas del acoplador y su efecto puede ser considerado como una reducción de la potencia de salida. La variación del pico de atenuación resulta de casi 8,5 \% con respecto al valor nominal.

En la Fig. 3.4(b), el valor pico del retardo de grupo aumenta ligeramente, como al variar las pérdidas de la guía de ondas, pero en forma casi imperceptible. Gracias a un acercamiento de la zona se pudo medir esta variación, que resultó del orden de $\pm 0,2 \%$. De 
esta manera se comprueba que la simplificación que se realiza en trabajos anteriores al no tener en cuenta las pérd idas del acoplador está justificada. También en este caso, aunque no se muestre en la figura, la periodicidad de la respuesta no se ve modificada, es decir, la distancia entre los picos de retardo no cambia con el aumento de las pérdidas.
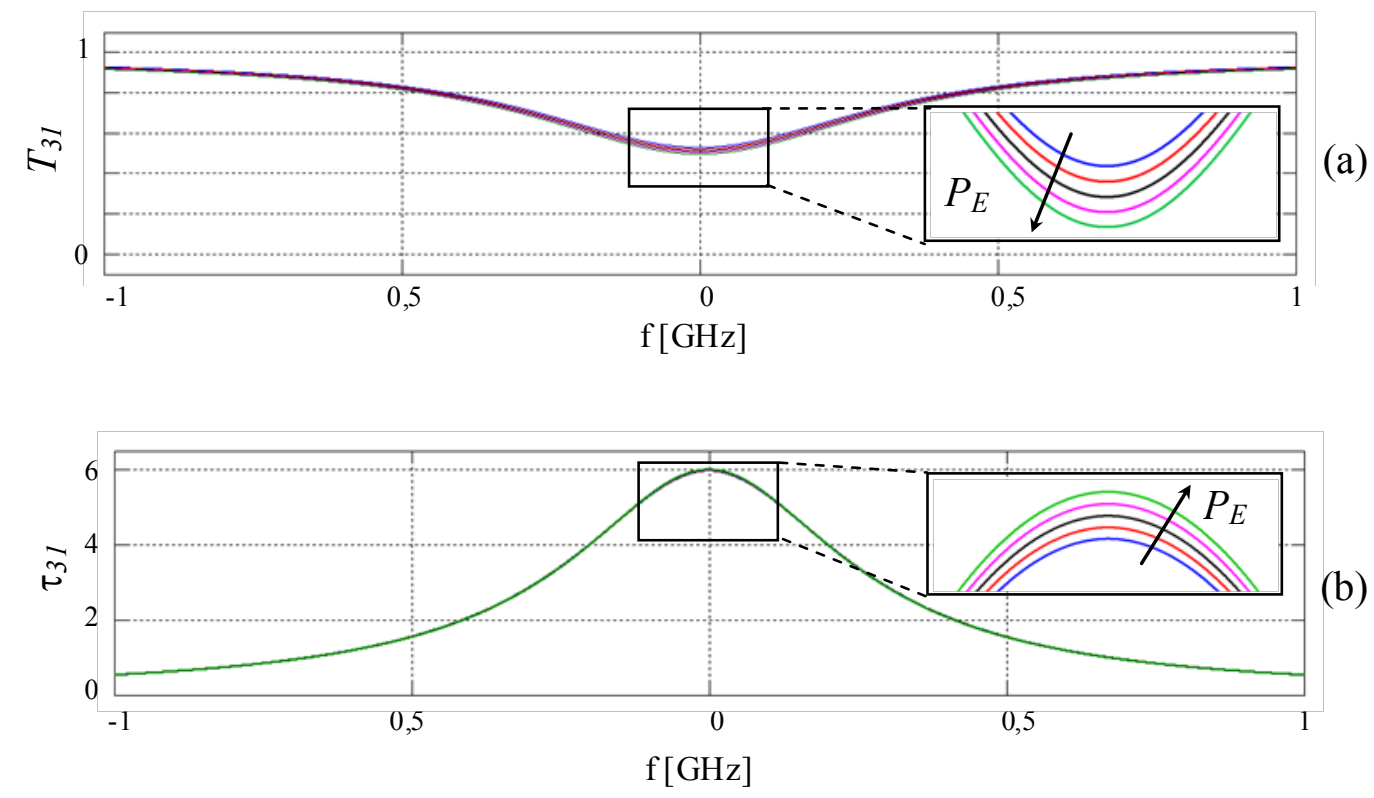

Figura 3.4: Variación de las pérdidas $\mathrm{P}_{\mathrm{E}}$ : (a) Coeficiente de transmisión de intensidad; (b) Retardo de grupo normalizado.

\section{Índice de refracción efectivo de la guía}

Para simular la variación del índice de refracción efectivo, $n_{e f}$, se consideraron valores del mismo en un entorno de 1,48 (una variación de $\pm 1,35 \%$ ). En particular, se utilizaron los valores: 1,$46 ; 1,47 ; 1,48 ; 1,49$ y 1,50. Los resultados se presentan en la Fig. 3.5, donde se observa que tanto el coeficiente de transmisión de intensidad como el retardo de grupo exhiben un desplazamiento progresivo de los picos de resonancia a medida que aumenta el índice efectivo de la guía. Esto se debe a que éste último es uno de los parámetros que determina la condición de resonancia (Ec. (3.3)). Sin embargo, para los valores de $n_{e f}$ considerados siempre existe un pico correspondiente a la longitud de onda de $1550 \mathrm{~nm}$ $(\Omega=0)$, y esto permite apreciar la reducción delFSR. 


$$
\theta_{r t}=\frac{2 \pi L n_{e f}}{\lambda}=2 \pi N \quad \rightarrow \quad L=\frac{\lambda N}{n_{e f}}, \quad N \in \mathbb{Z}
$$
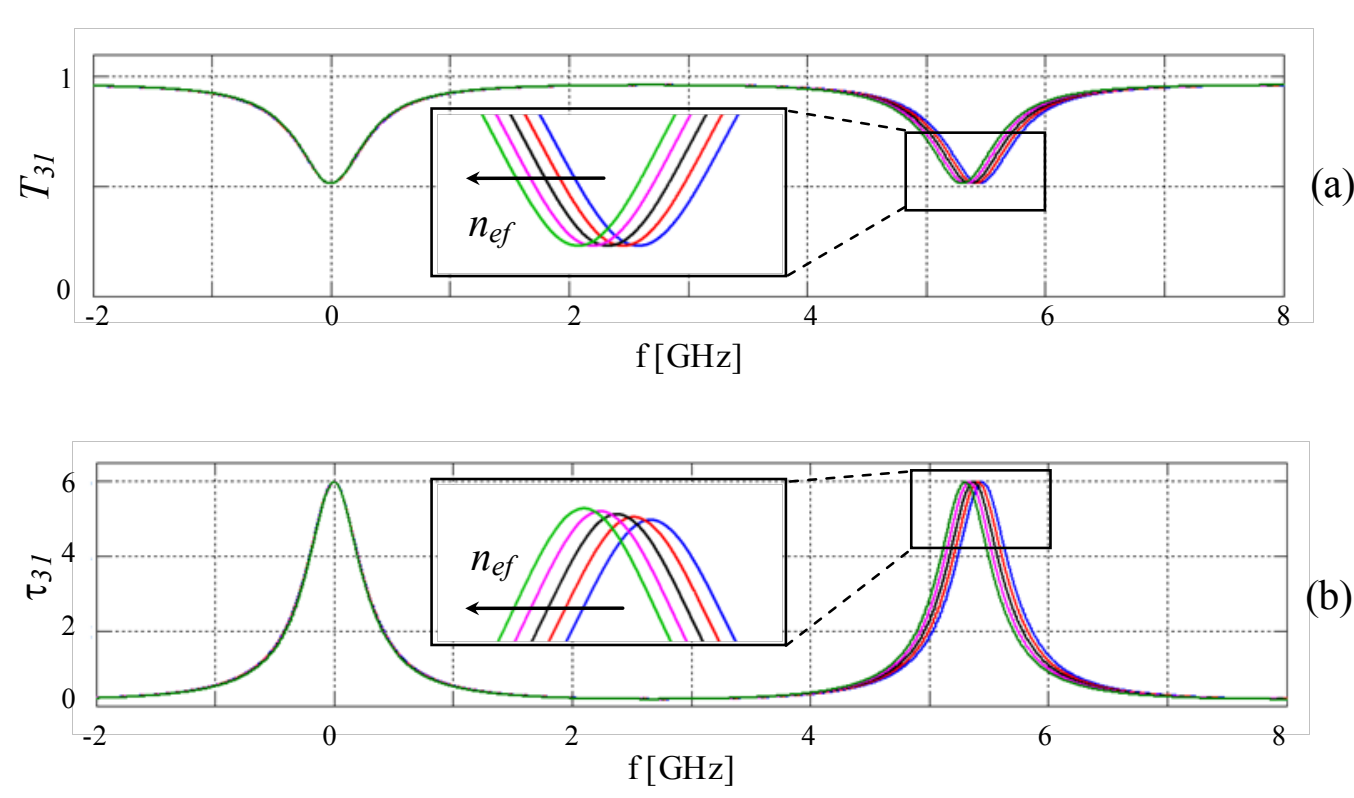

Figura 3.5: Variación del índice efectivo $n_{e f}$ : (a) Coeficiente de transmisión de intensidad; (b) Retardo de grupo normalizado.

En la Fig. 3.5(a) se observa que el pico de coeficiente de transmisión de intensidad sólo se desplaza en el eje de frecuencias, sin modificar su amplitud. El retardo de grupo, en la Fig. 3.5(b), se encuentra normalizado con respecto al mayor tiempo de round-trip y esto permite apreciar un ligero aumento del valor pico a medida que se incrementa el índice de refracción. Sin embargo, la variación del valor pico resultó menor a $\pm 1,4 \%$.

\section{Modulación de fase}

Como se observa en la Fig. 3.6, y de acuerdo con la Ec. (3.1), el efecto del modulador de fase dentro del anillo es el de desplazar en frecuencia la respuesta del dispositivo al modificar la frecuencia de resonancia. En particular, para un ORR con las características constructivas adoptadas, los desplazamientos de fase de $0 ; 0,2 \pi ; 0,4 \pi ; 0,6 \pi$ y $0,8 \pi$ simulados modifican la frecuencia entre 0 y $2,15 \mathrm{GHz}$. Esta variación podía preverse teniendo en cuenta que el FSR corresponde a una frecuencia normalizada de $2 \pi$ radianes y cuyo valor desnormalizado es de $5,38 \mathrm{GHz}$. 


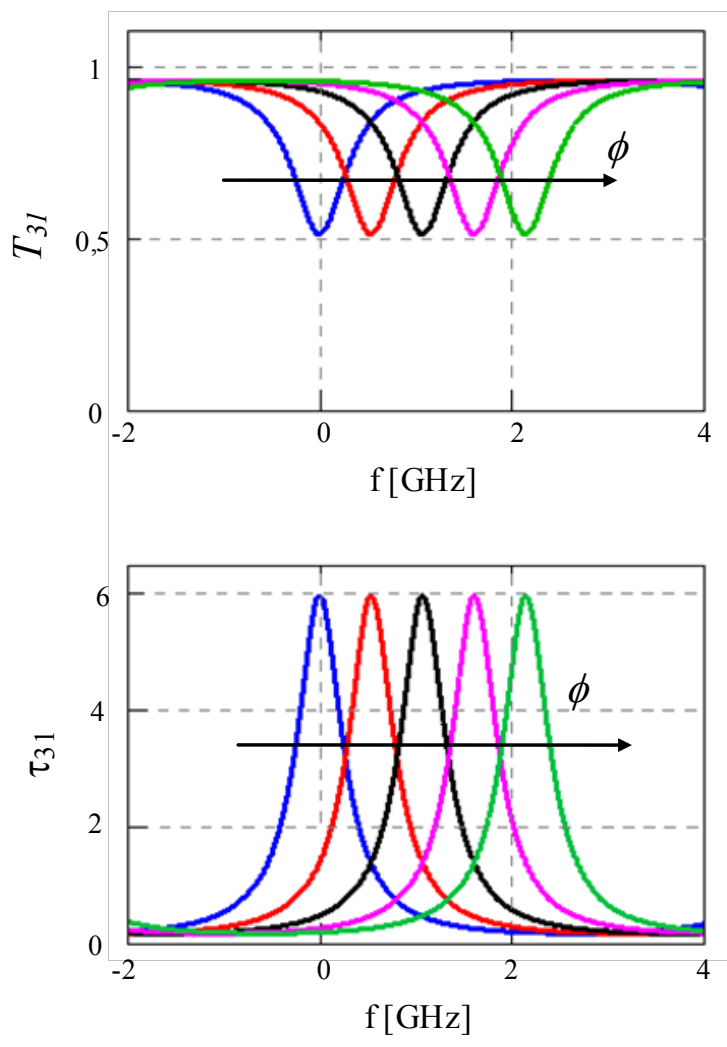

(a)

(b)

Figura 3.6: Variación de la fase adicional $\phi$ : (a) Coeficiente de transmisión de intensidad; (b) Retardo de grupo normalizado.

Sin embargo, la forma de la respuesta no se ve modificada. El coeficiente de transmisión de intensidad y el retardo de grupo normalizado mantienen, tanto el valor pico como el FWHM para todos los desplazamientos simulados. Este comportamiento puede resultar de utilidad en aplicaciones que requieran, por ejemplo, actuar sobre ciertas longitudes de onda y poder seleccionarlas, o cuando se requiera ajustar pequeñas desviaciones que puedan aparecer durante el proceso de fabricación o por cambios de temperatura durante el funcionamiento normal del dispositivo. Otra aplicación que para la cual este comportamiento resulta de utilidad es la implementación de retardos de mayor ancho de banda por medio de la conexión en cascada de varios resonadores sintonizados a distintas frecuencias de resonancia, como se verá más adelante. Cabe mencionar que, como cualquier dispositivo real, el modulador de fase introduce pérdidas que en este caso han sido incluidas en el parámetro de pérdidas de la guía de ondas.

A modo de resumen, en la Tabla 3.1 se comparan los resultados obtenidos para los distintos parámetros, teniendo en cuenta la capacidad de ajuste de los retardos (valor pico 
y/o frecuencia de resonancia) y el FSR, la variación del coeficiente de transmisión de intensidad en la banda de paso, y la dificultad de implementación.

TABLA 3.1

COMPARACIÓN DE LA INFLUENCIA DE LOS DISTINTOS PARÁMETROS DE UN ORR

\begin{tabular}{|c|c|c|c|c|c|c|}
\hline & $n_{e f}$ & $L$ & $P_{E}$ & $k$ & $\phi$ & $\alpha$ \\
\hline Capacidad de ajuste de $\tau_{31}$ & Baja & Alta & Baja & Alta & Alta & Baja \\
\hline Capacidad de ajuste del $F S R$ & Baja & Alta & No & No & No & No \\
\hline Variación de $T_{31}$ & Baja & Media & Baja & Alta & No & Baja \\
\hline Dificultad de implementación & Alta & Alta & Baja & Media & Media & Baja \\
\hline
\end{tabular}

\subsection{Conclusiones}

En este capítulo se realizaron simulaciones numéricas de la respuesta de un ORR que permitieron entender el efecto de la variación de cada uno de los parámetros que intervienen en la función de transferencia del dispositivo. Del análisis realizado se desprende que los parámetros que mayor influencia tienen sobre la respuesta son el factor de acoplamiento $k$, la modulación de fase $\phi$, y la longitud del anillo (a través del radio del mismo), mientras que las pérdidas de la guía de ondas, las del acoplador y el índice de refracción efectivo, si se mantienen dentro de sus valores típicos, no modifican la respuesta de manera apreciable.

En particular, el factor de acoplamiento y la modulación de fase actúan sobre el valor pico y la frecuencia de resonancia de la respuesta, respectivamente, y son los parámetros más adecuados para controlar los retardos y aumentar el ancho de banda del sistema, como se verá más adelante en el Capítulo 7.

\section{Referencias}

[1] D. A. P: Bulla, B. V. Borges, M. A. Romero, N. I. Morimoto, L. G. Neto and A.L. Cortes, "Design and fabrication of $\mathrm{SiO}_{2} / \mathrm{Si}_{3} \mathrm{~N}_{4} \mathrm{CVD}$ optical waveguides," International Microwave and Optoelectronics Conference, 1999. SBMO/IEEE MTT-S, APS and LEOS - IMOC '99. (Volume: 2).

[2] R. Chávez Velázquez, I. Zaldívar Huerta, C. Reyes Betanzo y A. Díaz Sánchez, "Fabricación de guías de onda ópticas en silicio utilizando óxido de silicio y nitruro de 
silicio", Sociedad Mexicana de Ciencia y Tecnología de Superficies y Materiales, Superficies y Vacío 18 (4), diciembre de 2005.

[3] A. Maalouf, C. Bastianelli, F. Mahé, A. Belmiloudi, M. Gadonna and D. Bosc, "Improved integrated resonators in polymer technology for tunable filter", Proc. SPIE 8431, Silicon Photonics and Photonic Integrated Circuits III, 843124 (June 1, 2012).

[4] L. Zhuang, D. Marpaung, M. Burla, W. Beeker, A. Leinse, and C. Roeloffzen, "Lowloss, high-index-contrast $\mathrm{Si}_{3} \mathrm{~N}_{4} / \mathrm{SiO}_{2}$ optical waveguides for optical delay lines in microwave photonics signal processing," Optics Express, Vol. 19, No. 23, 7 November 2011, pp. 23162-23170. 
Heinrich Sebastian Rabal - Líneas de Retardo Real Para Conformación Óptica de Haces de Microondas 


\section{Capítulo 4 - Acoplamiento crítico}

\subsection{Introducción}

En este capítulo se analiza un resonador óptico en anillo (ORR) en condición de funcionamiento crítica. El dispositivo fue descripto en capítulos anteriores, donde se analizó su funcionamiento, se obtuvo su función de transferencia (Ec. (4.1)), y se estudió la influencia de cada uno de los parámetros que intervienen en la misma.

La Fig. 4.1 muestra un esquema del dispositivo, cuyos parámetros de control son: el factor de acoplamiento $k$, y el desplazamiento de fase $\phi$ que introduce el modulador de fase, MF, dentro del anillo.

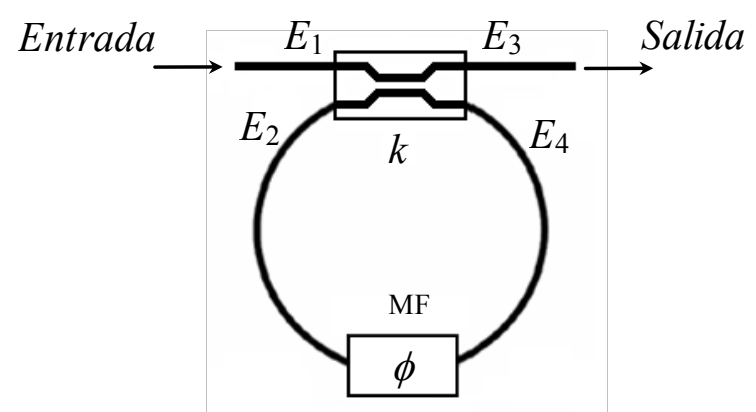

Figura 4.1: Esquema de un ORR controlable.

$$
t_{31}(\Omega)=\frac{E_{3}}{E_{1}}=\left[\frac{\xi \cdot \sqrt{1-k}-\xi^{2} \cdot \sqrt{a} \cdot e^{-j \phi} \cdot e^{-j \Omega}}{\left(1-\xi \cdot \sqrt{1-k} \cdot \sqrt{a} \cdot e^{-j \phi} \cdot e^{-j \Omega}\right)}\right]
$$

Como se desprende de los análisis anteriores, existe una condición de funcionamiento para la cual la transmisión de intensidad a la salida es nula a la frecuencia de resonancia y el retardo de grupo que introduce el dispositivo comienza a exhibir una respuesta anómala, 
con valores negativos. En las siguientes secciones se analiza con más detalle lo que sucede en esta condición tanto en el dominio de la frecuencia como en el plano complejo, y se obtiene la ecuación que permite determinar el valor del factor de acoplamiento crítico para unas dadas características constructivas del anillo.

\subsection{Va riación fina del factor de acoplamiento}

En las siguientes figuras se muestra en forma casi continua la variación de la respuesta (coeficiente de transmisión de intensidad, fase y retardo de grupo normalizado) en función del factor de acoplamiento $k$ con el fin de visualizar la evolución de la misma alrededor de la condición crítica.

Con color azul y rojo se indican las curvas correspondientes a las condiciones subacoplada y sobreacoplada respectivamente, mientras que con color negro y en línea punteada se indica la curva correspondiente al acoplamiento crítico, que en este caso es $k_{\mathrm{c}}=0,104$.

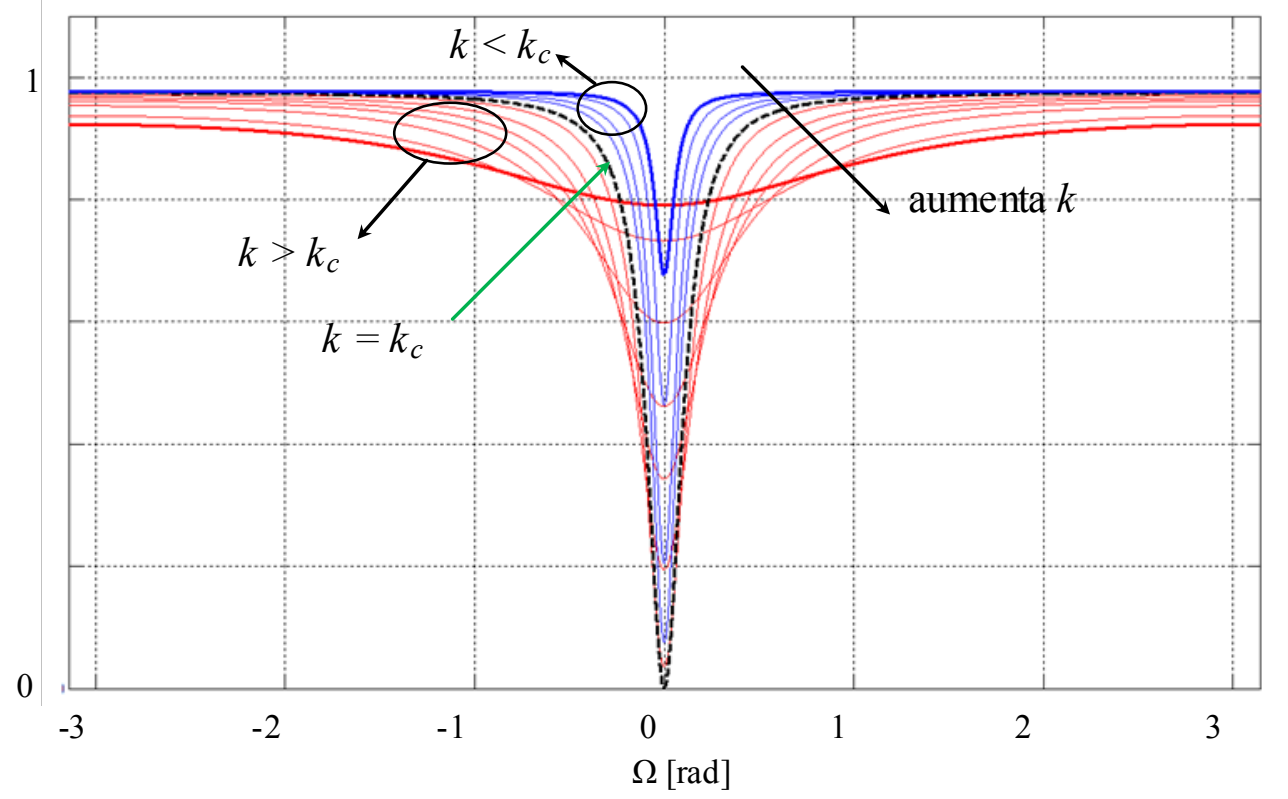

Figura 4.2: Variación fina del factor de acoplamiento: coeficiente de transmisión de intensidad.

Como puede verse en la Fig. 4.2, cuando el acoplamiento es crítico, el coeficiente de transmisión de intensidad es cero a la frecuencia de resonancia y el dispositivo se comporta como un filtro notch. 
Partiendo del menor valor de $k$, en la condición subacoplada, el pico de atenuación a la frecuencia de resonancia acentúa a medida que se incrementa el factor de acoplamiento, hasta llegar al acoplamiento crítico, para el cual no se transmite luz a la salida. A partir de dicho valor, un aumento en el factor de acoplamiento se traduce en una reducción de la atenuación, pero con una respuesta en frecuencia más ancha (FWHM), como se ve en la Fig. 4.2 indicado con color rojo.

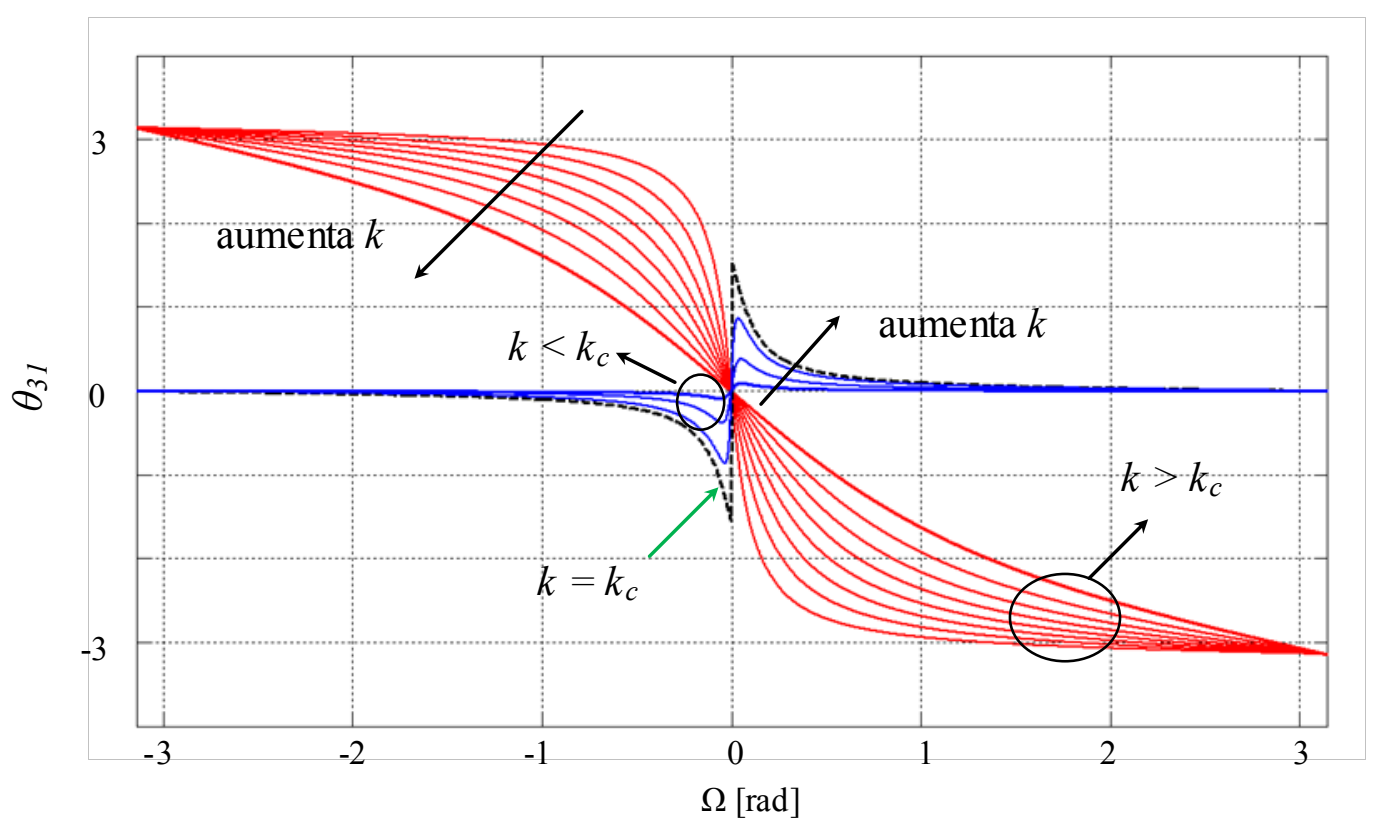

Figura 4.3: Variación fina del factor de acoplamiento: fase de la transferencia. 


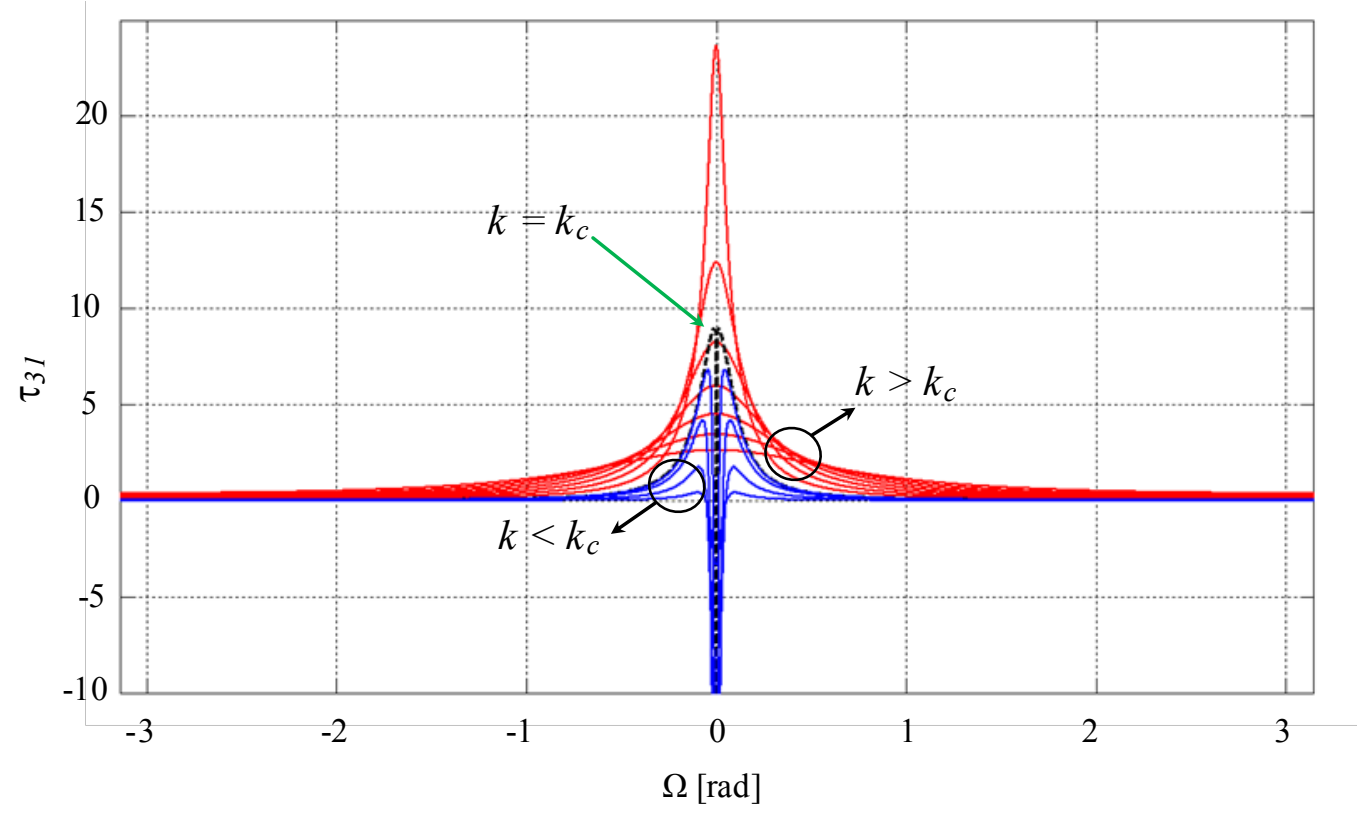

Figura 4.4: Variación fina del factor de acoplamiento: retardo de grupo normalizado.

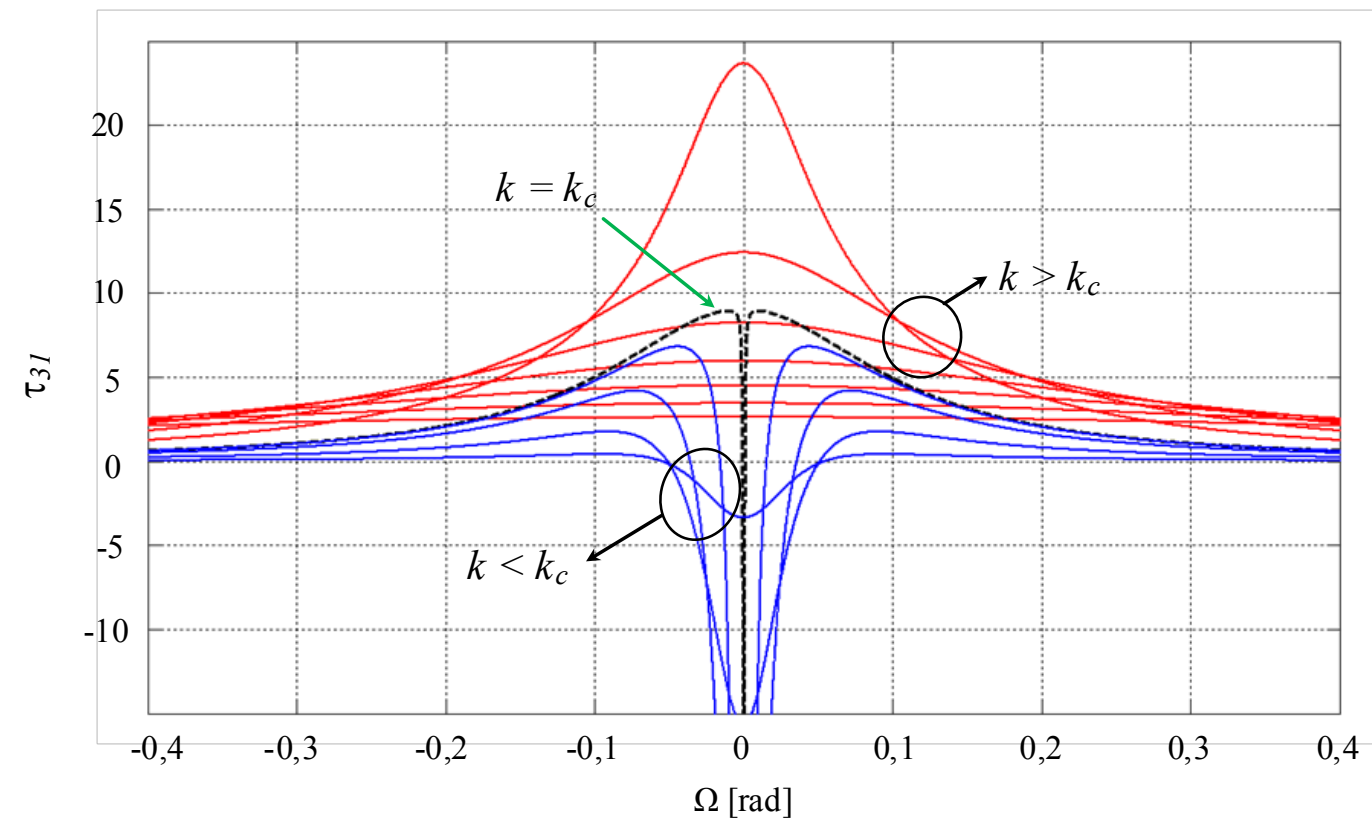

Figura 4.5: Retardo de grupo normalizado (acercamiento de la Fig 4.4).

La Fig. 4.3 muestra que en la condición de acoplamiento crítico la fase sufre un cambio de pendiente, que pasa de máxima positiva a máxima negativa. De acuerdo con la definición del retardo de grupo, y como se verifica gráficamente en la Fig. 4.4, esto significa que en un entorno de la frecuencia de resonancia, el retardo pasa de tener un valor 
máximo negativo a uno máximo positivo al incrementar el factor de acoplamiento. Un acercamiento en la Fig. 4.5 permite apreciar con más detalle el comportamiento del retardo de grupo.

El comportamiento que exhibe el retardo de grupo en la zona subacoplada, es decir para valores de $k$ menores al crítico, no resulta adecuado para implementar retardos de banda ancha. En esta condición existen tanto retardos positivos como negativos que producirían una distorsión en la señal que se transmita a través del dispositivo. Por el contrario, la zona sobreacoplada resulta más adecuada para la aplicación mencionada, ya que en esta zona todos los retardos resultan positivos y de amplitud y ancho de banda variables de acuerdo al valor de $k$.

\subsection{Análisis mediante la transformada $\mathrm{Z}$}

El valor del factor de acoplamiento crítico puede obtenerse mediante del análisis del diagrama de polos y ceros de la función de transferencia, haciendo uso de la Transformada Z. Reemplazando $\exp (-j \Omega)=\mathrm{z}^{-1}$ en la Ec. (4.1), se obtiene la función de transferencia en la variable $\mathrm{z}$ :

$$
t_{31}(z)=\left[\frac{\xi \sqrt{1-k}-\xi^{2} \sqrt{a} e^{-j \phi_{Z^{-1}}}}{\left(1-\xi \sqrt{1-k} \sqrt{a} e^{\left.-j \phi_{Z^{-1}}\right)}\right.}\right]
$$

Los polos y ceros de la transferencia (raíces del denominador y numerador, respectivamente) están dados por $z_{p}$ y $z_{c}$, y se obtienen a partir de la Ec. (4.2):

$$
\begin{gathered}
z_{p}=\xi \sqrt{1-k} \sqrt{a} e^{-j \phi} \\
z_{c}=\frac{\xi \sqrt{a} e^{-j \phi}}{\sqrt{1-k}}
\end{gathered}
$$

Las Ecs. (4.3) y (4.4) muestran que el módulo de los polos y ceros depende del factor de acoplamiento $k$, mientras que la fase es la misma para ambos y está determinada por el parámetro $\phi$.

Es pertinente hacer notar que la Ec. (4.3) ind ica que para un dispositivo real, con valores de $a, \xi$ y $k$ menores a 1 , los polos siempre estarán ubicados dentro del círculo unitario, haciendo al dispositivo inherentemente estable. Esto puede modificarse si se introduce dentro del anillo un elemento con ganancia suficiente como para superar a las pérdidas, de 
hecho existen trabajos en los que se analiza dicha situación [1-3]. Sin embargo, este trabajo está enfocado en dispositivos resonadores pasivos, sin ganancia dentro del anillo.

En la Fig. 4.6 se muestra el diagrama de polos y ceros de un ORR con las mismas características constructivas que en los análisis previos para distintas condiciones de acoplamiento sobreacoplado, es decir con factor de acoplamiento mayor al crítico $\left(k>k_{c}\right)$ y con el parámetro $\phi$ fijo en el valor $-\pi / 6$. En la misma figura, en los correspondientes recuadros interiores se muestran el coeficiente de transmisión de intensidad y el retardo de grupo.

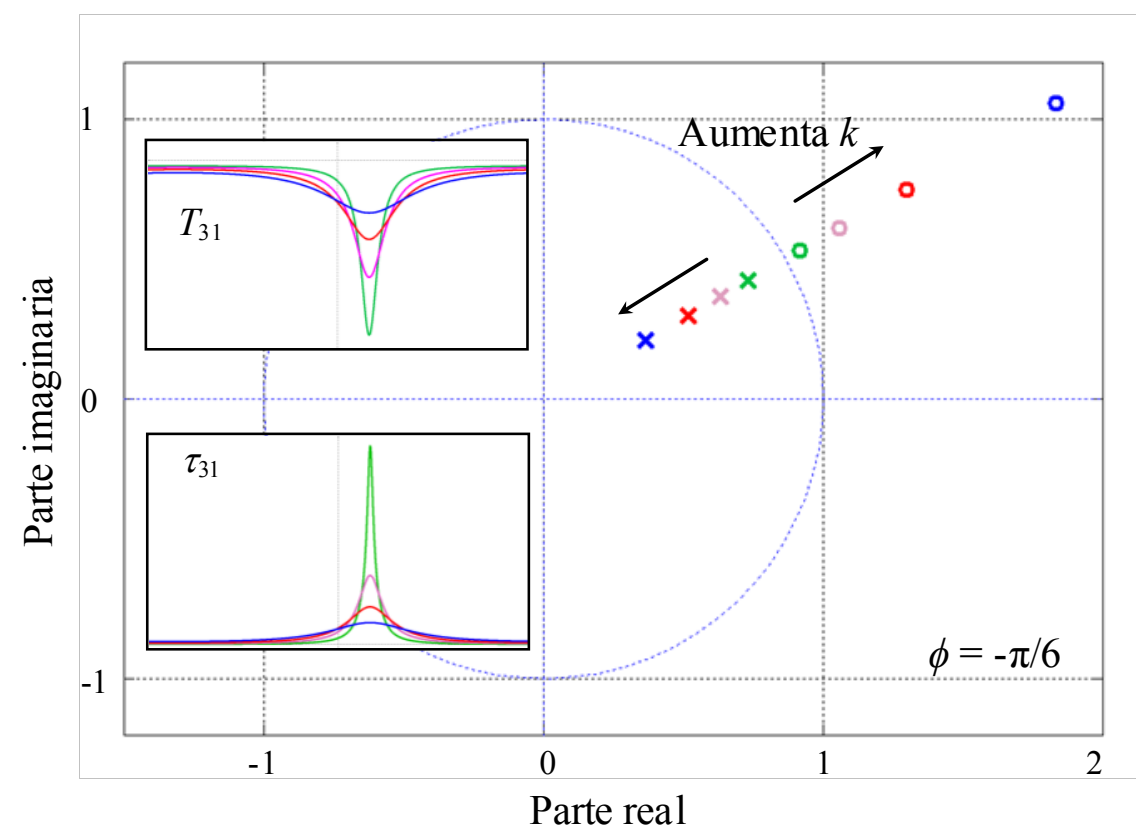

Figura 4.6: Diagrama de polos y ceros de un ORR al variar el factor de acoplamiento $k=0,2 ; 0,4 ; 0,6$ y $0,8 .\left(\alpha=10 \mathrm{~dB} / \mathrm{m}, P_{\mathrm{E}}=0,1 \mathrm{~dB}, \phi=-\pi / 6\right)$.

Cuando se reduce el factor de acoplamiento desde su máximo valor, el polo y el cero se desplazan acercándose al círculo unitario. Si se continúa reduciendo $k$, es posible alcanzar la condición de acoplamiento critico $\left(k=k_{c}\right)$, para la cual, idealmente, la transmisión de intensidad es cero a la frecuencia de resonancia. Esta condición se alcanza cuando el modulo del cero $z_{c}$ tiende a 1 , es decir:

$$
\left|z_{c}\right|=1 \quad \rightarrow \quad \frac{\xi \sqrt{a}}{\sqrt{1-k_{c}}}=1
$$


Y el factor de acoplamiento crítico resulta:

$$
k_{c}=1-\xi^{2} a
$$

En la Fig. 4.7 se muestra el diagrama de polos y ceros cuando el cero de la función de transferencia alcanza el círculo unitario, es decir en condición de acoplamiento crítico (símbolos rojos). Si se reduce aún más el factor de acoplamiento, el dispositivo funciona en una condición subacoplada $\left(k<k_{c}\right)$ en la que tanto el polo como el cero de la función de transferencia se encuentran dentro del círculo unitario (símbolos azules). En el límite, cuando el factor de acoplamiento es cero, la luz $\mathrm{n}$ o se acopla al anillo y se transmite por completo hacia el puerto de salida sin ningún retardo. En esta condición, el polo y el cero colapsan en el punto $z=z_{c}=z_{p}$.

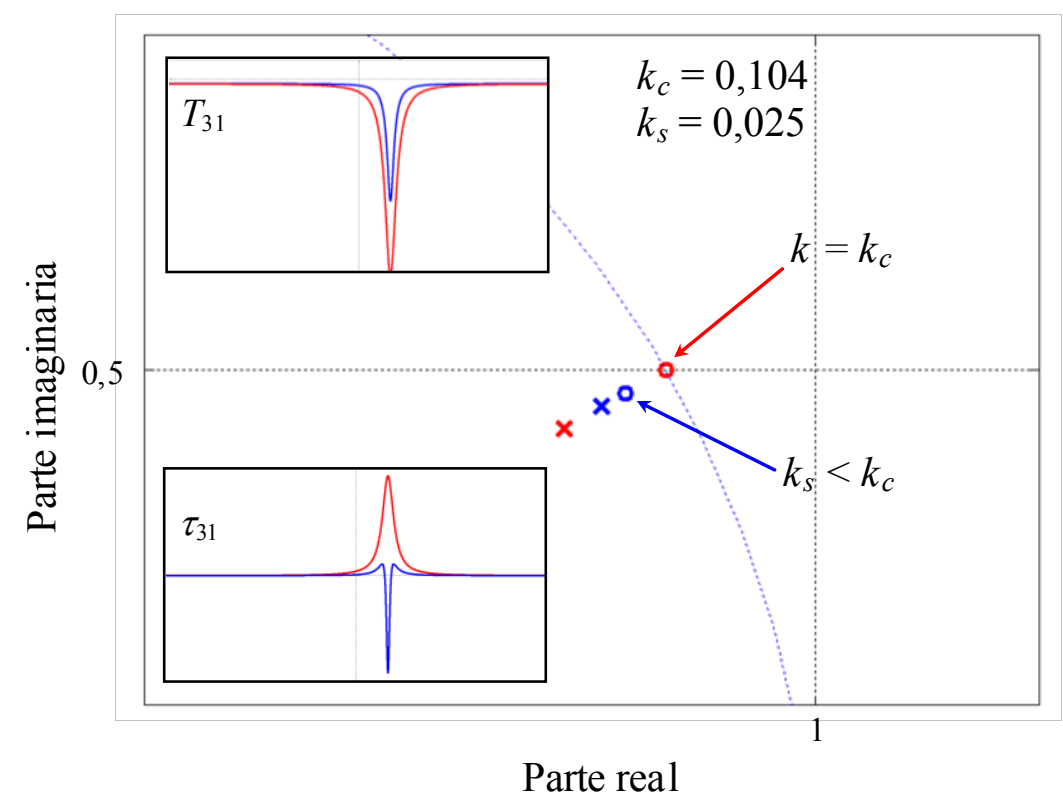

Figura 4.7: Diagrama de polos y ceros en dos condiciones: acoplamiento crítico $\left(k=k_{c}\right)$ y condición subacoplada $\left(k<k_{c}\right)$. 


\subsection{Conclusiones}

Se analizó la respuesta de un ORR en un entorno de la condición de acoplamiento crítica y se mostró que dicha condición está determinada por la relación entre las pérdidas (todas ellas) y el factor de acoplamiento, $k$. Este análisis permitió definir dos zonas de trabajo con características de retardo bien diferenciadas. Por un lado, una zona subacoplada en la que el retardo exhibe una forma anómala, con retardos positivos y negativos en una banda estrecha de frecuencias. Estas irregularidades en el retardo producen una gran distorsión en la señal que se propaga por el dispositivo, por lo que la zona subacoplada no resulta adecuada para implementar retardos de banda ancha. Por otro lado, en la zona de trabajo sobreacoplada, el retardo es positivo para todas las frecuencias y exhibe una forma de campana más o menos suave dependiendo del valor del factor de acoplamiento. Esta zona sí resulta apropiada para generar grandes retardos controlados, pero debe tenerse en cuenta la relación de compromiso que existe entre el valor pico y el ancho de banda de los mismos. Para satisfacer los requerimientos de ancho de banda, puede recurrirse a alguna técnica o estrategia que permita sortear este obstáculo, como se explicará más adelante.

\section{Referencias}

[1] P. A. Costanzo Caso; S. Granieri; A. Siahmakoun, "Optical Leaky Integrator with Inverted and Non-inverted Accumulation," Microwave and Optical Technology Letters, Vol. 53, 2011, pp. 2034-2037.

[2] Hsien-kai Hsiao and K. A. Winick "Planar glass waveguide ring resonators with gain," Optics Express, Vol. 15, No. 26, 24 December 2007, pp. 17783-17797.

[3] P. A. Costanzo-Caso, Y. Jin, S. Granieri and A. Siahmakoun "Optical bistability in a Nonlinear SOA-based Fiber Ring Resonator,” Journal of Nonlinear Optical Physics \& Materials, Vol. 20, No. 3, 2011, pp. 281-292. 


\section{Capítulo 5 - ORR: Propagación de un pulso}

\subsection{Introducción}

En este capítulo se analiza la propagación de un pulso de luz de banda angosta (por ej. láser) a través del resonador óptico en anillo (ORR) estudiado en los capítulos previos. Las simulaciones de propagación se realizaron para distintas condiciones de acoplamiento del ORR, en las tres zonas de funcionamiento posibles: subacoplada, crítica, y sobreacoplada.

El objetivo es visualizar el efecto del retardo de grupo que introduce el dispositivo, así como la atenuación de intensidad a la salida. Además, las simulaciones permiten verificar si se produce algún tipo de distorsión en el pulso que dificulte su detección posterior.

\subsection{Características del pulso de entra da}

Se eligió un pulso, $P_{\text {in }}(\mathrm{t})$, con forma gaussiana para modular una fuente láser de longitud de onda coincidente con la de resonancia del ORR y con ancho de línea despreciable, de manera de aprovechar todo el ancho de banda del dispositivo y la simetría de la respuesta, ya que de esta manera las componentes frecuenciales del pulso se ven igualmente afectadas a cada lado de la frecuencia de resonancia.

La duración temporal del pulso, si se define como el ancho a mitad del máximo (FWHM), es de 11,7 ns, y la potencia pico normalizada a 1. En la Fig. 5.1 se muestra el pulso de entrada en el dominio del tiempo y se indica su duración. 


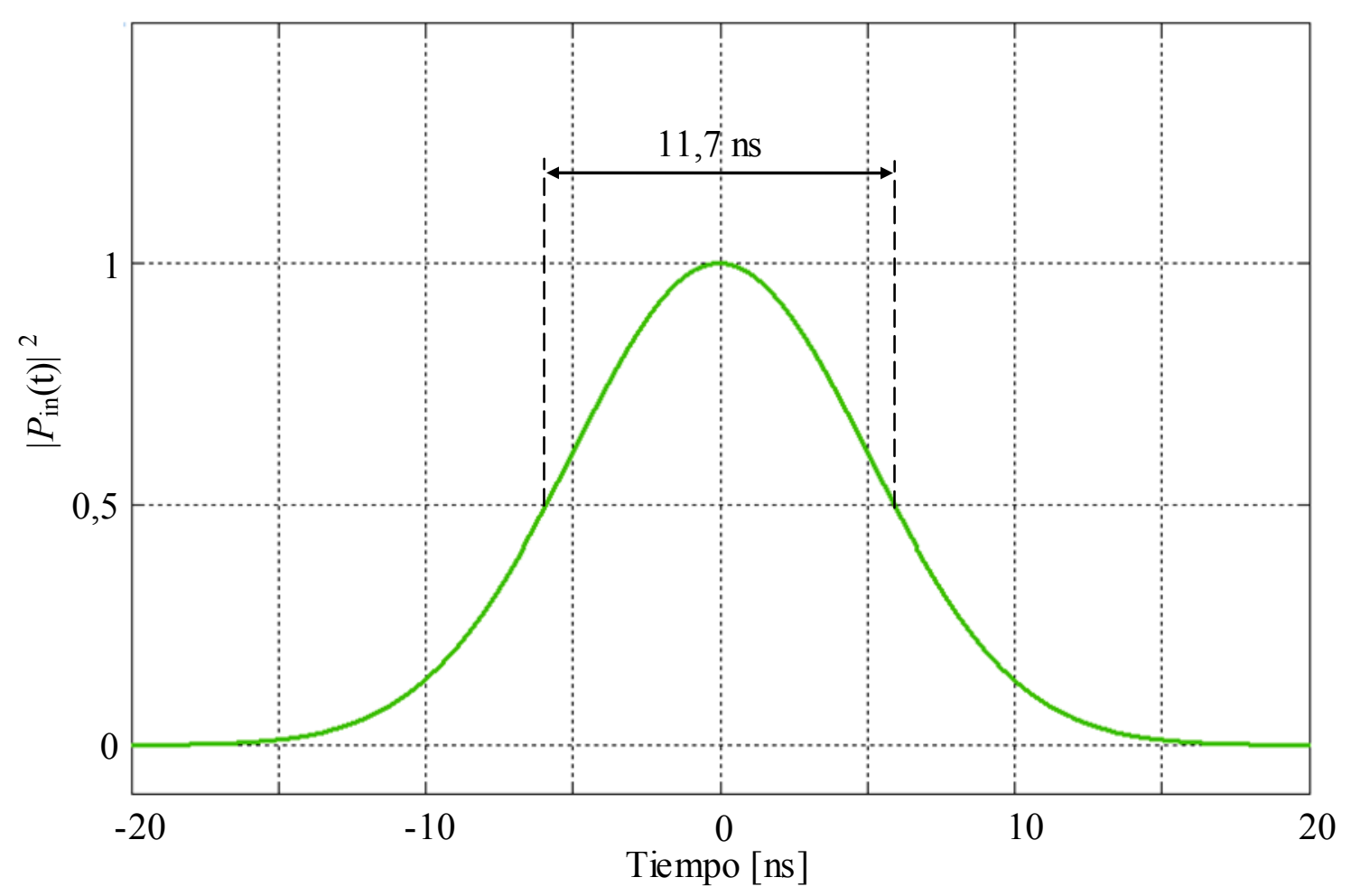

Figura 5.1: Potencia del pulso de entrada en el dominio del tiempo.

\subsection{Pulso de salida}

Si $P_{\text {in }}(\Omega)$ representa la transformada de Fourier del pulso de entrada (en este caso obtenida mediante el algoritmo de la FFT (Fast Fourier Transform) en Matlab $\left.{ }^{\circledR}\right)$ y $t_{31}(\Omega)$ la función de transferencia del ORR dada por la Ec. (5.3), el pulso de salida, $P_{\text {out }}(t)$, puede obtenerse de la siguiente manera:

$$
\begin{gathered}
P_{\text {out }}(\Omega)=P_{\text {in }}(\Omega) \cdot T_{31}(\Omega) \\
P_{\text {out }}(t)=F^{-1}\left\{P_{\text {out }}(t)\right\} \\
t_{31}(\Omega)=\frac{E_{3}}{E_{1}}=\left[\frac{\xi \sqrt{1-k}-\xi^{2} \sqrt{a} \cdot e^{-j \phi} e^{-j \Omega}}{\left(1-\xi \sqrt{1-k} \cdot \sqrt{a} \cdot e^{-j \phi} e^{-j \Omega}\right)}\right]
\end{gathered}
$$

Donde $F^{-1}\{\}$ indica la Transformada Inversa de Fourier.

Debido a que la salida del fotodetector con el que se recibe la señal en la práctica es proporcional a la potencia del pulso, en la siguiente sección se visualiza el módulo al cuadrado de $P_{\text {in }}(\mathrm{t})$ y $P_{\text {out }}(\mathrm{t})$ para distintas condiciones de acoplamiento. 


\subsection{Simulacio nes $y$ a nálisis}

En las siguientes figuras se muestra la potencia del pulso de salida comparada con la de entrada para distintas condiciones de acoplamiento elegidas en forma arbitraria, y abarcando las zonas sobreacoplada y subacoplada: $k=0,8 ; 0,5 ; 0,3 ; 0,2 ; 0,15 ; 0,104$ (valor crítico); 0,08; 0,02 y 0,002. Para mayor claridad, en la Fig. 5.2 se presentan los casos correspondientes a valores de $k$ entre 0,8 y 0,2, mientras que en la Fig. 5.3 se muestran aquellos que corresponden a valores de $k$ entre 0,2 y 0,002 .

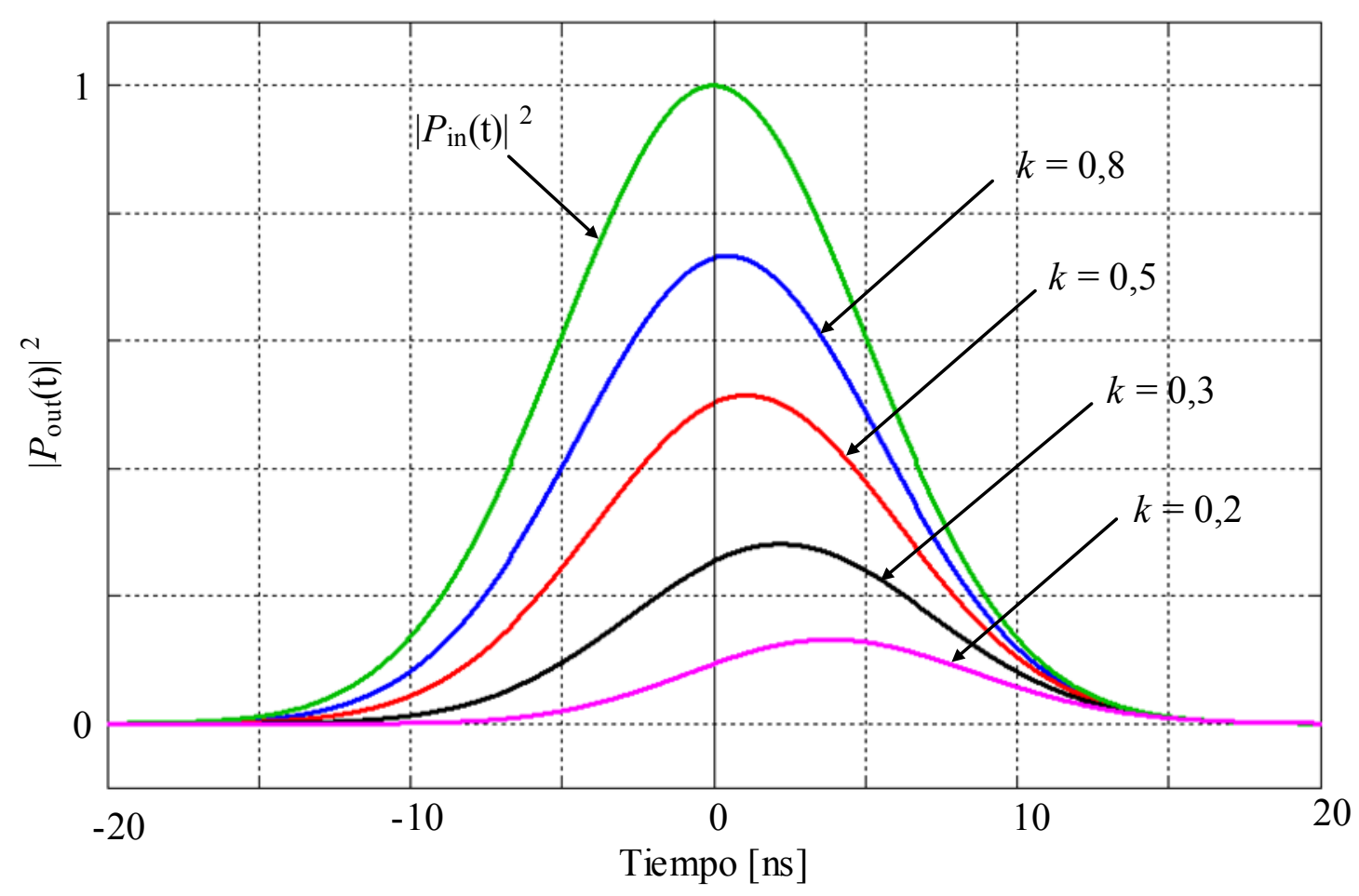

Figura 5.2: Potencia del pulso de salida para $k=0,8 ; 0,5 ; 0,3$ y 0,2 .

En primer lugar, en la Fig. 5.2 se observa que la amplitud del pulso de salida se reduce a medida que el factor de acoplamiento $k$ disminuye desde su valor inicial, 0,8 . Es to coincide con lo esperado de acuerdo a la variación del coeficiente de transmisión de intensidad analizada en los capítulos previos.

También puede observarse el efecto del retardo de grupo en el pico del pulso de salida, que aparece retardado con respecto al del pulso de entrada. El retardo observado es mayor a medida que el factor de acoplamiento se reduce, como se analizó en la sección correspondiente. 


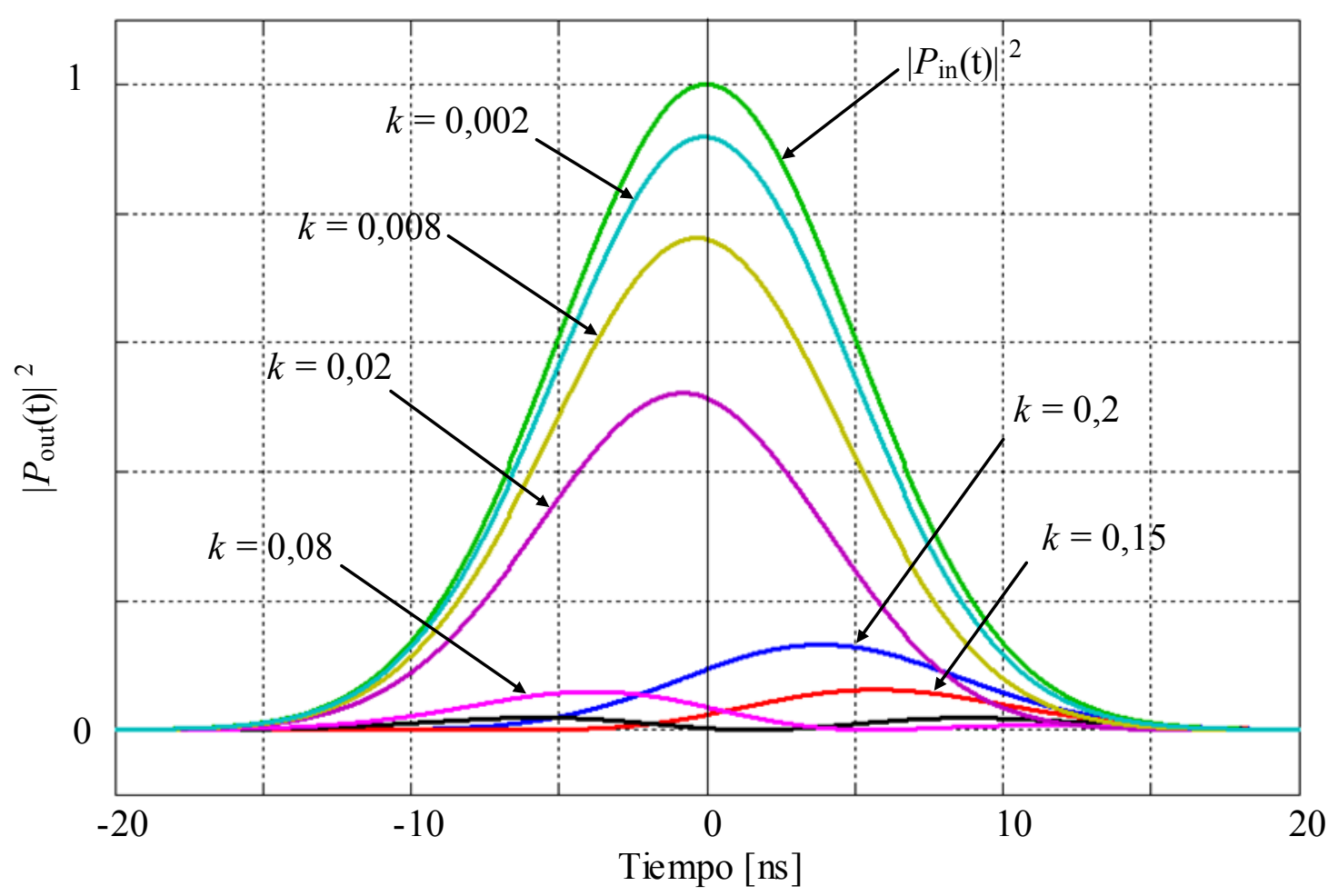

Figura 5.3: Potencia del pulso de salida para $k=0,2 ; 0,104 ; 0,15 ; 0,08 ; 0,02$; 0,008 y 0,002 .

En la Fig. 5.3, donde se muestran los casos correspondientes a valores de $k$ menores a 0,2 , se observa que al seguir reduciendo el factor de acoplamiento, existe una condición en la que el pulso de salida comienza a exhibir lo que parece un retardo negativo, y se ve 'adelantado' con respecto al pulso de entrada. Un acercamiento (Fig. 5.4) permite ver que cuando el factor de acoplamiento alcanza su valor crítico $\left(k_{\mathrm{c}} \sim 0,104\right)$, el pulso se degenera en dos. Esto se debe a que el retardo de grupo en función de la frecuencia presenta una forma como la de la Fig. 5.5, donde se observan zonas de retardo positivo y zonas de retardo negativo. De esta manera, algunas componentes frecuenciales del pulso, en particular las que se encuentran cerca de la frecuencia de resonancia, se ven afectadas por un retardo negativo, mientras que las más alejadas sufren un retardo positivo. 


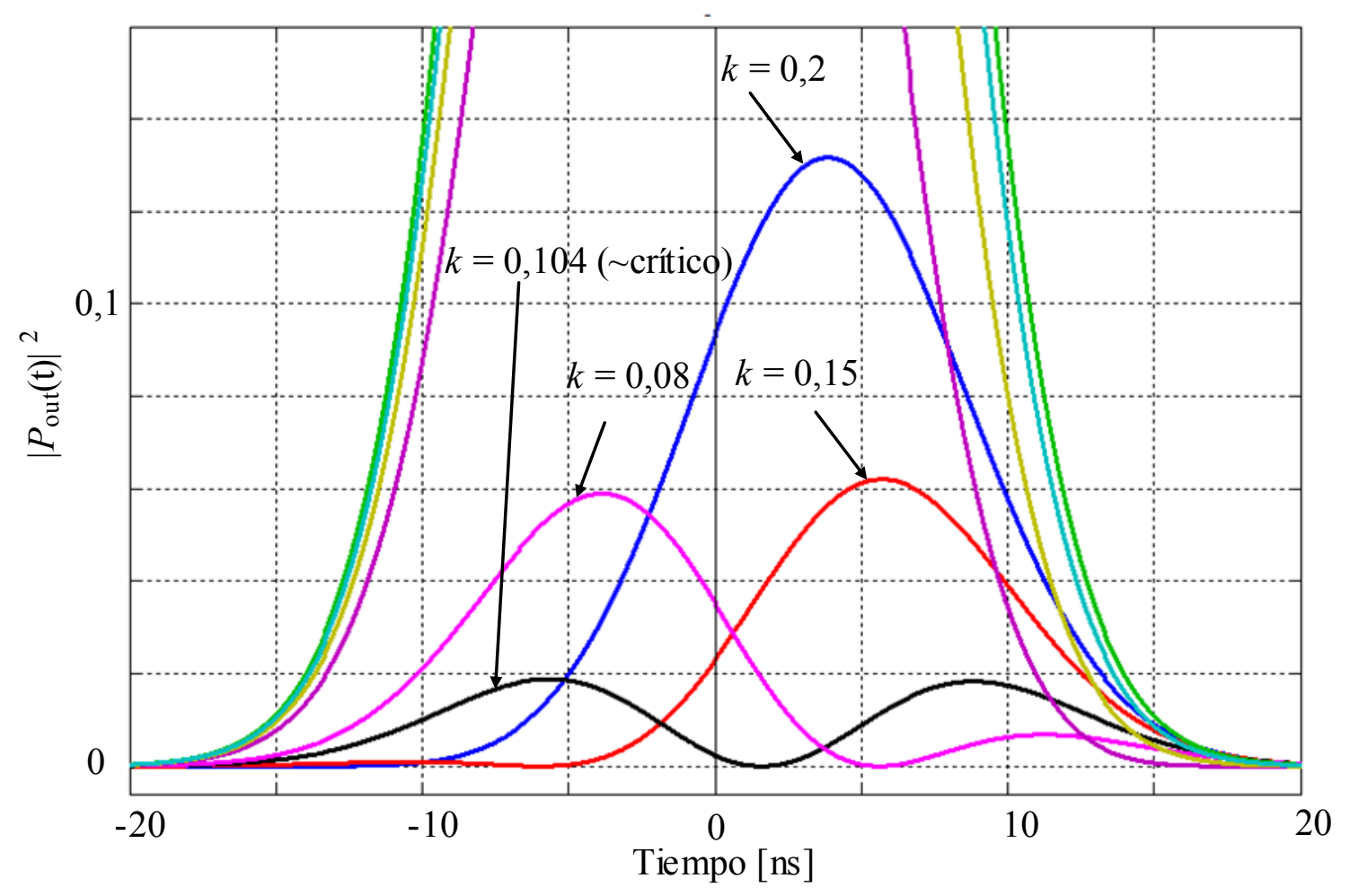

Figura 5.4: Potencia de salida para la condición subacoplada (acercamiento de la Fig. 5.3).

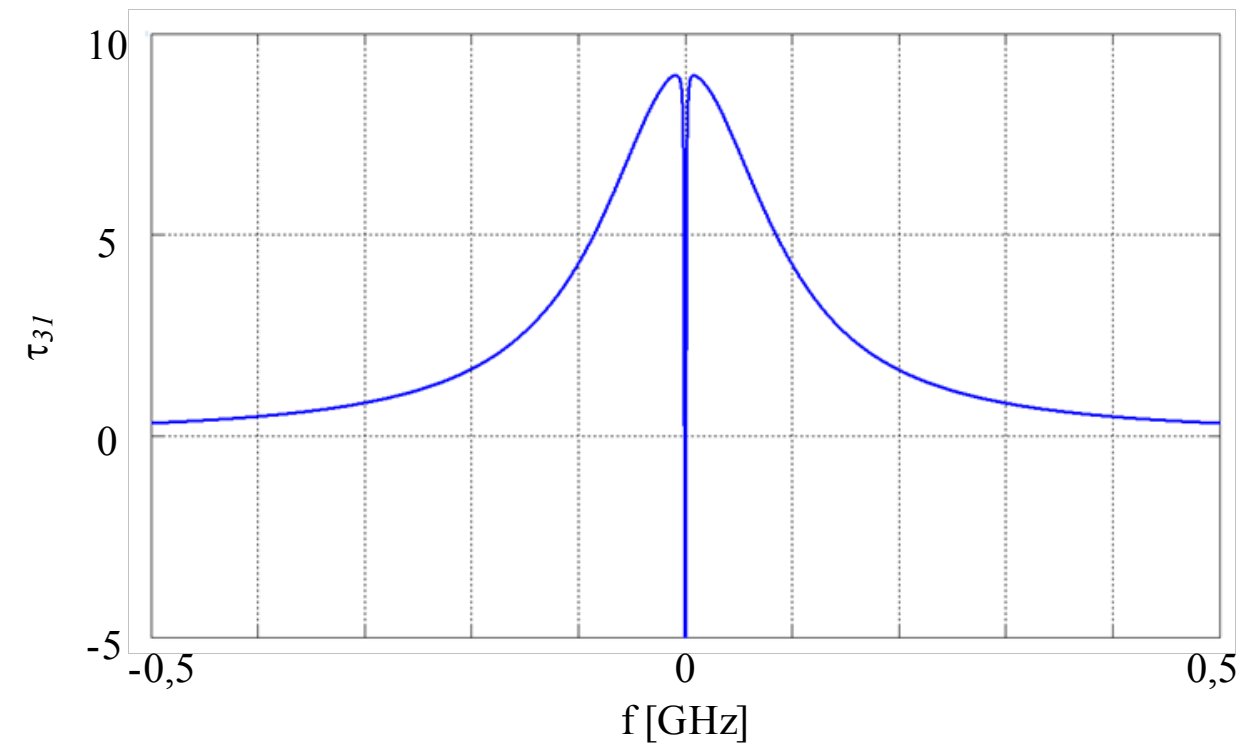

Figura 5.4: Retardo de grupo normalizado para $k=0,104$, con zonas positivas y negativas. 
Sin embargo, no debe perderse de vista que lo que se ve 'adelantado' es el pico del pulso, y esto se debe a una redistribución de la energía del mismo. En realidad, el pulso de salida nunca antecede al de entrada, como ha sido demostrado experimentalmente en [1], donde se analiza la propagación de un pulso a través de un ORR y se obtienen gráficos muy similares a los presentados en este capítulo. De esta manera, se verifica que no existe una contradicción con el principio de causalidad. Además, este comportamiento anómalo no es propio de los ORR, sino de ciertos tipos de filtros [2] y ya ha sido estudiado antes. Sin embargo, un análisis más detallado del funcionamiento en la zona subacoplada escapa a los objetivos de este trabajo, que está enfocado en la implementación de retardos positivos de gran ancho de banda.

\subsection{Conclusiones}

En esta sección se analizó la propagación de un pulso de luz a través de un resonador óptico en anillo (ORR) con el fin de verificar cualitativamente el efecto del retardo de grupo que predicen las simulaciones de los capítulos previos. Se realizaron simulaciones para distintas condiciones de acoplamiento del ORR, y en particular se diferenciaron las zonas de trabajo sobreacoplada, crítica y subacoplada, observándose en este último caso el efecto del retardo negativo con respecto a la portadora óptica, en forma de 'adelantamiento' del pico del pulso de salida con respecto al del pulso de entrada.

Los resultados que se observaron en este capítulo permiten justificar la elección de la zona sobreacoplada como zona de trabajo del dispositivo en los siguientes capítulos, en los que se estudiarán distintas variaciones y tipos de conexión con el objetivo de mejorar las características del retardo, como son el valor pico y el ancho de banda.

\section{Referencias}

[1] H. P. Uranus, L. Zhuang, C.G.H. Roeloffzen, and H.J.W.M. Hoekstra, "Direct Experimental Observation of Pulse Temporal Behavior in Integrated-Optical RingResonator with Negative Group Velocity," European Conference on Integrated Optics, ECIO 2007, Post Deadline Papers, 25-27 April 2007, Copenhagen, Denmark (pp. FPD1/1-FPD1/4) 
[2] Radan Slavík and Jǐrí C` tyroký, "Light Advancement and Delay by Linear Filters With Close to Zero Resonant Transmittance," Journal Of Lightwave Technology, Vol. 26, No. 23, December 1, 2008. pp. 3708-3713. 
Heinrich Sebastian Rabal - Líneas de Retardo Real Para Conformación Óptica de Haces de Microondas 


\section{Capítulo 6 - ORR: Configuración de 4 puertos}

\subsection{Introducción}

En este capítulo se estudia una configuración alternativa de los resonadores ópticos presentados en los capítulos anteriores. En esta nueva configuración, parte de la luz que resuena dentro del anillo es acoplada a una segunda guía de ondas a través de un acoplador adicional. De esta manera, se obtiene un dispositivo de cuatro puertos: dos de entrada y dos de salida, como se muestra en la Fig. 6.1.

Aunque no es el objetivo de este trabajo, se puede mencionar una posible aplicación de estos dispositivos como selector de canales en los sistemas ópticos de comunicaciones en los cuales se transmiten varios canales por una misma fibra óptica, como son los sistemas multiplexados en longitud de onda (Wavelength Division Multiplexing (WDM)). En estos sistemas muchas veces es necesario utilizar filtros muy selectivos y angostos, ya sea para separar los canales o para reducir el ruido en el caso de transmitir un único canal, y los ORRs han demostrado ser una opción que cumple con los requerimientos [1-3]. En la Fig. 6.1 se ejemplifica este tipo de aplicación.

En las siguientes secciones de este capítulo, se analizará la respuesta del dispositivo en relación con la implementación de líneas de retardo de banda ancha. 


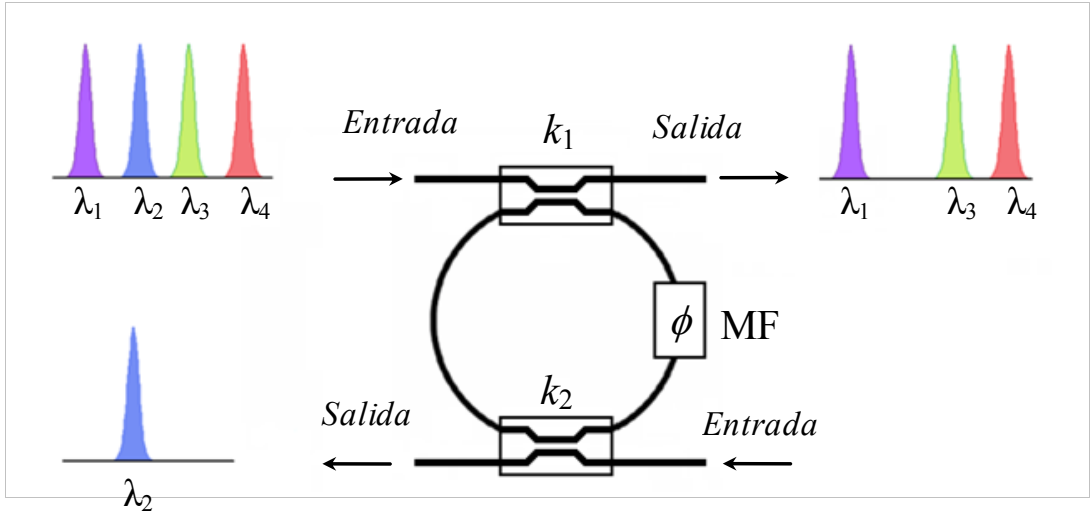

Figura 6.1: ORR de cuatro puertos y su aplicación como filtro de canales WDM.

\subsection{Descripción del siste ma}

En el esquema de la Fig. 6.2 se indican los puertos 1 y 4 , de entrada, y 2 y 3 , de salida, así como los campos eléctricos que intervienen en cada uno de ellos. El dispositivo tiene dos acopladores, representados por $k_{1} \mathrm{y} k_{2}$. El primero cumple la misma función que en un anillo simple de dos puertos, mientras que el segundo permite acoplar parte de la luz que resuena en la cavidad a una segunda guía recta y obtener así el campo eléctrico $E_{7}$ en la salida del Puerto 3.

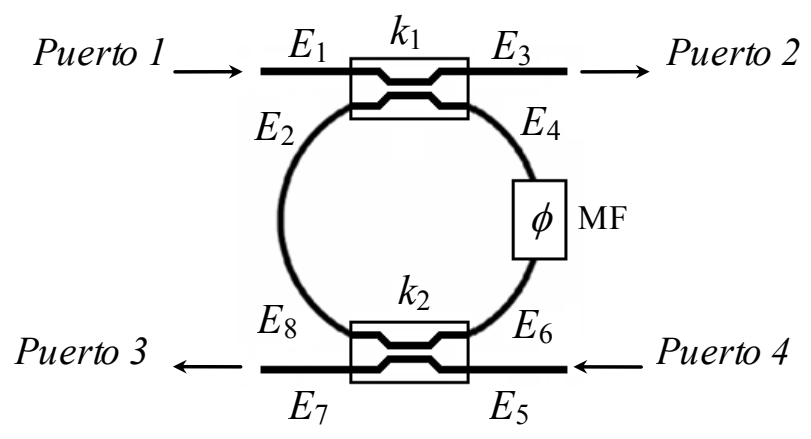

Figura 6.2: Esquema de un ORR de cuatro puertos. 


\section{Función de transferencia}

Los elementos que componen el dispositivo son los mismos que en un resonador de dos puertos, con el agregado del segundo acoplador dentro del anillo. A partir de las ecuaciones que describen el funcionamiento de los acopladores y la propagación en la guía de onda, y luego de algunas operaciones algebraicas, se obtienen las funciones de transferencia que relacionan las entradas con las salidas del dispositivo. En este caso, las Ecs. (6.1) y (6.4) relacionan las salidas con la entrada del Puerto 1.

$$
\begin{gathered}
t_{31}(\Omega)=\frac{E_{3}}{E_{1}}=\left[\frac{\xi_{1} \cdot \sqrt{1-k_{1}}-\xi_{1}{ }^{2} \cdot \xi_{2} \cdot \sqrt{1-k_{2}} \cdot \sqrt{a} \cdot e^{-j \Omega} e^{-j \phi}}{\left(1-\xi_{1} \cdot \sqrt{1-k_{1}} \cdot \xi_{2} \cdot \sqrt{1-k_{2}} \cdot \sqrt{a} \cdot e^{-j \Omega} e^{-j \phi}\right)}\right] \\
t_{71}(\Omega)=\frac{E_{7}}{E_{1}}=e^{j(\pi-\Omega / 2-\phi)} \cdot\left[\frac{\xi_{1} \cdot \xi_{2} \cdot \sqrt[4]{a} \cdot \sqrt{k_{1}} \cdot \sqrt{k_{2}}}{\left(1-\xi_{1} \cdot \xi_{2} \cdot \sqrt{1-k_{1}} \cdot \sqrt{1-k_{2}} \cdot \sqrt{a} e^{-j \Omega} e^{-j \phi}\right)}\right]
\end{gathered}
$$

Debido a la simetría del dispositivo, las funciones de transferencia $t_{31}(\Omega)$ y $t_{75}(\Omega)$ resultan iguales, pero con los subíndices de los parámetros intercambiados, y lo mismo sucede con las transferencias $t_{71}(\Omega)$ y $t_{35}(\Omega)$. De forma que:

$$
\begin{aligned}
& E_{3}=t_{31}(\Omega) \cdot E_{1}+t_{35}(\Omega) \cdot E_{5} \\
& E_{7}=t_{71}(\Omega) \cdot E_{1}+t_{75}(\Omega) \cdot E_{5}
\end{aligned}
$$

Como se explicara en el Capítulo 2 para un ORR de dos puertos, en cada caso el coeficiente de transmisión de intensidad y el retardo de grupo normalizado se obtienen como el módulo de la función de transferencia elevado al cuadrado y la derivada cambiada de signo de la fase, $\theta(\Omega)$, con respecto a la frecuencia angular normalizada, respectivamente.

$$
\begin{gathered}
T(\Omega)=|t(\Omega)|^{2} \\
\tau=-d \theta(\Omega) / d \Omega
\end{gathered}
$$




\subsection{Análisis de la respuesta}

\section{Variación de los factores de acoplamiento}

Con el fin de analizar los cambios que produce en la respuesta del dispositivo la variación de los factores de acoplamiento $k_{1} \mathrm{y} k_{2}$, se realizaron simulaciones del coeficiente de transmisión de intensidad y el retardo de grupo correspondientes a las funciones de transferencia $t_{31}$ y $t_{71}$. En este caso no se presentan los resultados correspondientes a la variación del resto de los parámetros que intervienen en las ecuaciones (6.1) y (6.2) debido a que son similares a los que se observaron en el análisis del Capítulo 3.

En cuanto a las características constructivas del resonador, se consideraron las mismas que las utilizadas en los análisis previos, es decir:

- Pérdidas por exceso del acoplador: $\quad P_{E}=0,1 \mathrm{~dB}(\xi \cong 0,9886)$

- Coeficiente de atenuación de la guía: $\alpha=10 \mathrm{~dB} / \mathrm{m}$

- Radio del anillo: $\quad r=0,006 \mathrm{~m}$

- Índice efectivo de la guía: $\quad n_{\mathrm{ef}}=1,48$

La modulación de fase se eligió iguala 0 rad debido a que, como se vio en elCapítulo 3, tiene el efecto de modificar la frecuencia de resonancia sin afectar la forma de la respuesta. Con respecto a los rangos de variación de los factores de acoplamiento, se eligieron tres valores para $k_{1}$ y una variación más fina para el nuevo acoplador, $k_{2}$. En particular:

- Factor de acoplamiento $k_{1}=0,2 ; 0,4$ y 0,6

- Factor de acoplamiento $k_{2}=0,1$ (curva de color rojo) a 0,9 (curva de color azul) en pasos de 0,1 . 


\section{Función de transferencia hacia el Pue rto 2}

Al comparar la función de transferencia $t_{31}$, presentada en la Ec. (6.1), con la Ec. (2.6), correspondiente a un resonador de dos puertos, puede observarse que los parámetros del acoplador adicional intervienen modificando las pérdidas de la guía de onda con un factor igual a $\xi_{2}\left(1-k_{2}\right)^{1 / 2}$. Por lo tanto, los gráficos que se obtienen al simular la variación de $k_{2}$ exhiben el mismo comportamiento que los correspondientes a la variación de las pérdidas $\alpha$ en el Capítulo 3. La única diferencia es que en este caso el rango de variación es mayor, ya que la inclusión del segundo acoplador equivale a utilizar un coeficiente de atenuación de la guía de ondas de valor $\alpha^{\prime}$ (Ec. (6.7)), que para los valores adoptados de $\xi_{2}$ y $k_{2}$, varía entre $(24,78+\alpha)$ y $(277,90+\alpha) \mathrm{dB} / \mathrm{m}$.

$$
\alpha^{\prime}=\left(4,343 \cdot \ln \left\{\xi_{2}^{2}\left(1-k_{2}\right)\right\}+\alpha\right) \quad[d B / m]
$$

Por esta razón, a modo ilustrativo, en la Fig. 6.3 sólo se presenta uno de los casos simulados: el correspondiente a $k_{1}=0,2$. Allí se observa que el coeficiente de transmisión de intensidad alcanza un valor prácticamente cero a la frecuencia de resonancia cuando el factor de acoplamiento $k_{2}$ es igual a 0,1 (curva de color rojo). En esa condición, el retardo de grupo exhibe la forma anómala descripta en el Capítulo 4, pero no puede hablarse de un único valor de acoplamiento crítico, ya que la condición de acoplamiento crítico en este caso depende de los dos factores de acoplamiento que intervienen en la función de transferencia del ORR.
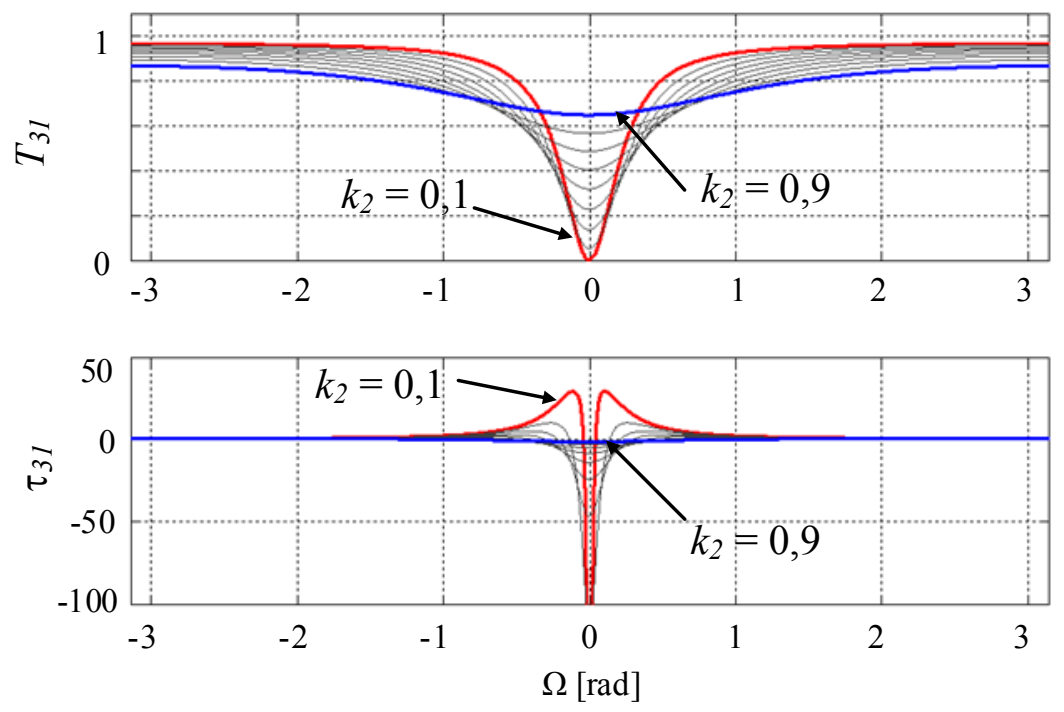

Figura 6.3: Transmisión de intensidad y retardo normalizado en el Puerto $2, k_{1}=0,2$. 


\section{Función de transferencia hacia el Pue rto 3}

A continuación, se presentan los resultados correspondientes a la función de transferencia $t_{71}$ que relaciona la entrada del Puerto 1 con la salida del Puerto 3 (ver Fig. 6.2). Para los tres valores de $k_{1}$ analizados, se observa que el coeficiente de transmisión de intensidad presenta una forma de campana, centrada en la frecuencia de resonancia, inversa a la que exhibe el coeficiente de transmisión de intensidad de la transferencia $t_{31}$. Esto significa que aquellas frecuencias que son atenuadas a la salida del Puerto 2, son transmitidas, por lo menos parcialmente, a la salida del Puerto 3.

El retardo de grupo, por otro lado, muestra una forma similar al de la transferencia $t_{31}$ debido a que la luz presente en la salida del Puerto 3 también resuena en la cavidad. En los tres casos simulados, el valor pico del retardo se reduce al aumentar el valor de $k_{2}$, en contraste con lo que sucede en un ORR de dos puertos, donde el valor pico aumenta a medida que se reduce el factor de acoplamiento, hasta llegar al valor crítico.

En particular, en la Fig. 6.4 se presenta el caso en que $k_{1}=0,2$. Como puede verse, a medida que el factor $k_{2}$ aumenta, también lo hace el pico de transmisión, pero reduciendo la selectividad de la respuesta. Este comportamiento debe tenerse en cuenta al utilizar el dispositivo en aplicaciones que requieran respuestas muy selectivas, como por ejemplo en un selector de canales WDM. El valor pico de transmisión es de 0,61, y se da para $k_{2}=0,3$.

En cuanto al retardo de grupo, el valor pico alcanza un valor máximo de 4,35 $\mathrm{T}_{\mathrm{RT}}$, que equivale a 809,77 ps y se reduce a medida que aumenta $k_{2}$ hasta $0,86 \mathrm{~T}_{\mathrm{RT}}(159,93 \mathrm{ps}) . \mathrm{El}$ ancho de la respuesta, caracterizado por el FWHM desnormalizado, varía desde 0,40 GHz hasta $2,72 \mathrm{GHz}$ para el rango de valores simulados. 

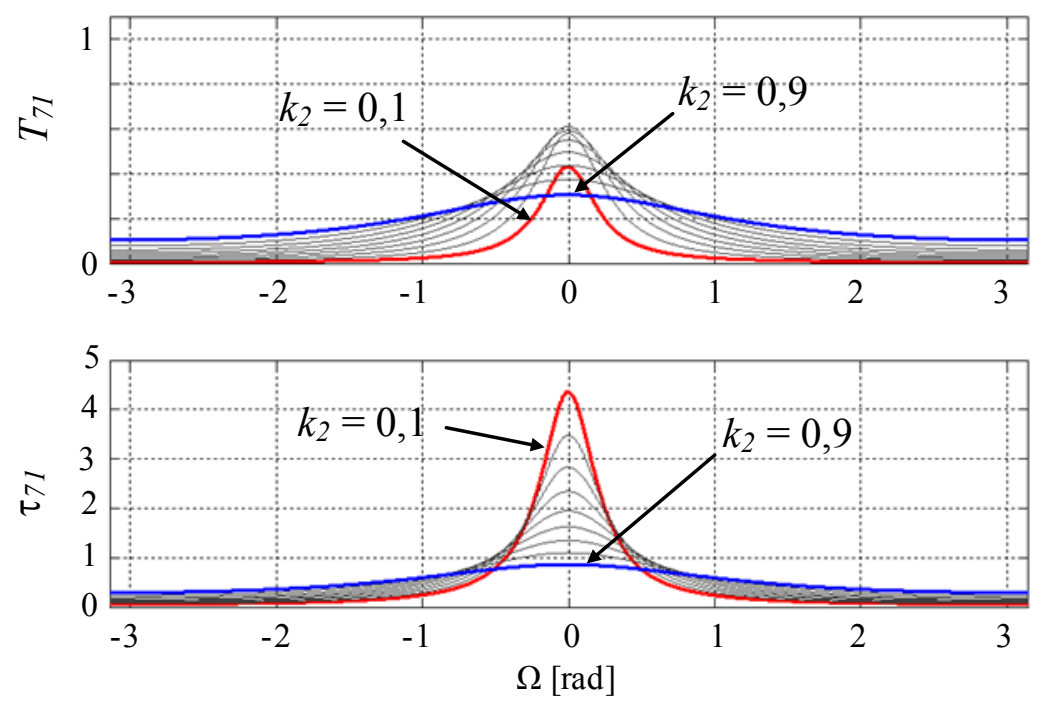

Figura 6.4: Transmisión de intensidad y retardo normalizado en el Puerto $3, k_{1}=0,2$.

En la Fig. 6.5 se muestra el caso en que $k_{1}=0,4$. En comparación con el caso anterior, el comportamiento es similar, pero la respuesta es menos selectiva, tanto en el coeficiente de transmisión de intensidad como en el retardo de grupo. El valor máximo para el pico de transmisión es 0,77 , se da para $k_{2}=0,5$, y es mayor que para $k_{1}=0,2$ analizado previamente. Por otro lado, el retardo el pico se reduce de 502,34 ps para $k_{2}=0,1$, a 148,30 ps para $k_{2}=0,9$. La reducción de la selectividad se verifica al medir el FWHM, que en este caso varía entre 0,83 y 3,20 GHz.
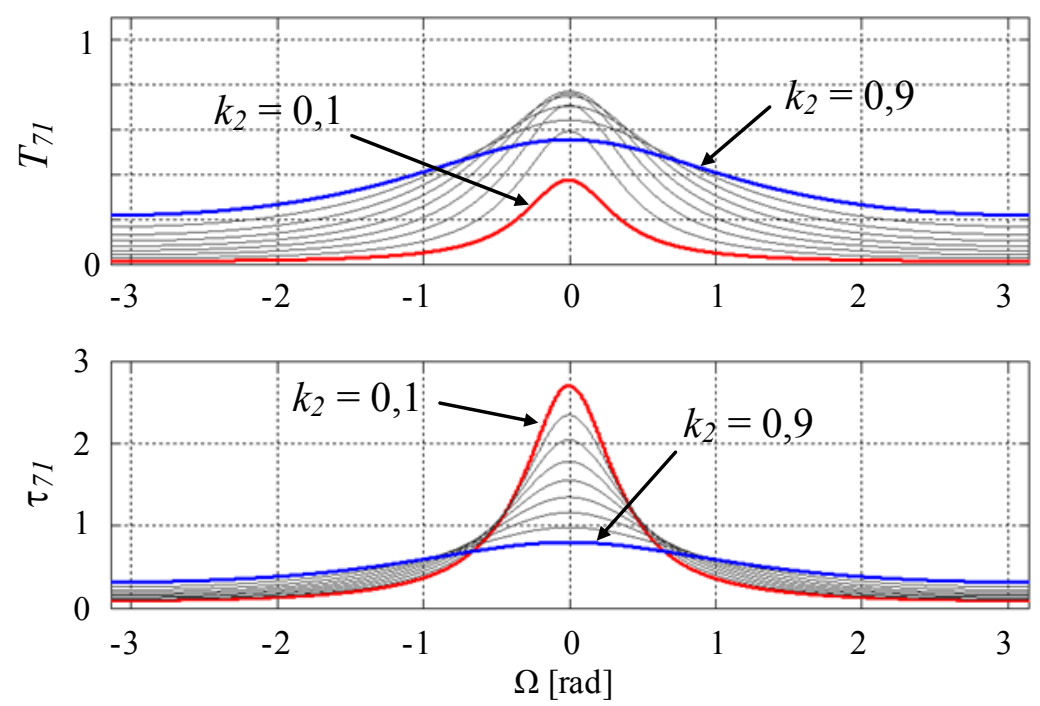

Figura 6.5: Transmisión de intensidad y retardo normalizado en el Puerto $3, k_{1}=0,4$. 
Por último, en la Fig. 6.6 se muestra el tercer caso, en el que $k_{1}=0,6$. Allí se observa un gran ensanchamiento de la respuesta. El coeficiente de transmisión de intensidad es mucho menos selectivo que en los casos anteriores, y alcanza un valor pico mayor, de alrededor de 0,8. Esto significa que más luz se transmite a la salida y con mayor intensidad. El retardo de grupo, sin embargo, aumenta su FWHM pero con valor pico menor, es decir que el ancho de banda es mayor que en los casos anteriores, pero se obtienen retardos de menor valor. En particular, el valor pico del retardo, desnormalizado, varía entre 331,05 y $135,80 \mathrm{ps,}$ mientras que el FWHM aumenta de 1,02 a más de $4 \mathrm{GHz}$, correspondientemente.
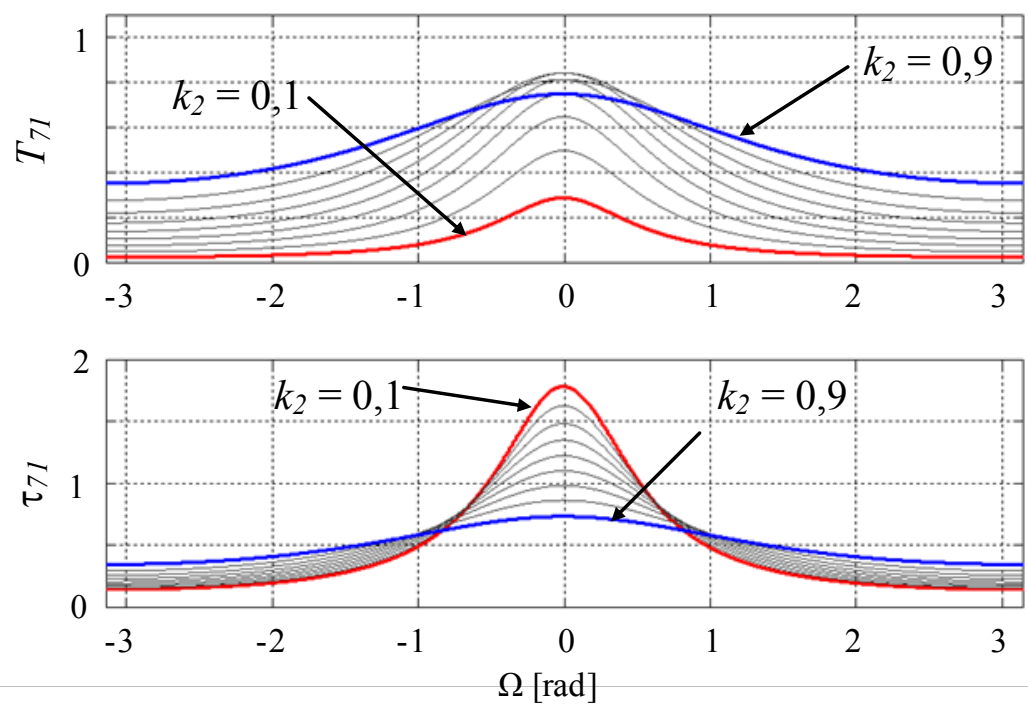

Figura 6.6: Transmisión de intensidad y retardo normalizado en el Puerto $3, k_{1}=0,6$.

Cabe destacar que en los tres casos analizados se observa que el valor pico del retardo de grupo normalizado toma, en ciertas condiciones, valores menores a 1. Esto no sucede en un resonador de dos puertos, donde el menor valor que puede tomar el pico del retardo dentro de la zona sobreacoplada es 1 y se da para $k=1$. En ese caso, toda la luz se acopla al anillo $\mathrm{y}$ al puerto de salida luego de un tiempo de round-trip. En cambio en el resonador de cuatro puertos, el pico de retardo se reduce hasta 0,5 para $k_{2}=1$, indicando que la luz recorre medio anillo antes de acoplarse al puerto de salida. 


\subsection{Conclusiones}

En este capítulo se presentó una variante del dispositivo resonador estudiado en los capítulos previos y se analizaron sus prestaciones relacionadas con la generación de retardos. Al agregar una segunda guía de ondas recta acoplada al anillo, el resonador se transforma en un dispositivo de cuatro puertos, uno de los cuales es de salida y permite seleccionar la parte del espectro de la señal de entrada que es atenuado en el otro. Se analizó particularmente esta señal de salida, luego de presentar un modelo matemático, obtenido de la misma manera que para el resonador de dos puertos.

Con el fin de caracterizar el comportamiento de la respuesta frente a variaciones de los parámetros de control, se realizaron simulaciones numéricas a partir del modelo matemático. Debido a que la influencia de algunos parámetros constructivos es similar a la que se observó al estudiar la respuesta del resonador de dos puertos, sólo se simularon variaciones de los dos factores de acoplamiento que intervienen en la respuesta del dispositivo.

Los gráficos obtenidos permitieron verificar que parte de la luz que resuena dentro de la cavidad se transmite hacia el puerto de salida adicional. El coeficiente de transmisión de intensidad en función de la frecuencia muestra en este caso forma de campana centrada en la frecuencia de resonancia y de selectividad variable en función de los factores de acoplamiento. El retardo de grupo, por otro lado, resulta similar al generado en el puerto de salida de un ORR de dos puertos, pero menos selectivo y con menor valor pico. En el rango de acoplamientos simulados, el valor máximo de retardo obtenido fue de 4,35 $\mathrm{T}_{\mathrm{RT}}$ (809,77 ps), con FWHM de 0,40 GHz.

En resumen, a mayores valores de $k_{1}$ y $k_{2}$ corresponden respuestas más anchas, con mayor transmisión de intensidad a la salida, pero con menor valor pico de retardo.

Si se comparan estos valores con los observados en los capítulos anteriores para un ORR de dos puertos, queda claro lo siguiente: si se desean obtener retardos de gran valor (entre 6 y $20 \mathrm{~T}_{\mathrm{RT}}$ ) la configuración de dos puertos resulta más apropiada, pero a costa de tener que utilizar alguna estrategia para aumentar el ancho de banda. Esto será demostrado en el Capítulo 7. 


\section{Referencias}

[1] Otto Schwelb, "Transmission, Group Delay, and Dispersion in Single-Ring Optical Resonators and Add/Drop Filters - A Tutorial Overview," Journal Of Lightwave Technology 22, 1380- (2004).

[2] B. E. Little, S. T. Chu, H. A. Haus, J. Foresi, and J.-P. Laine "Microring Resonator Channel Dropping Filters," Journal Of Lightwave Technology, Vol. 15, No. 6, June 1997, pp. 998-1005.

[3] B. E. Little,J. S. Foresi, G. Steinmeyer, E. R. Thoen, S. T. Chu, H. A. Haus, E. P. Ippen, L. C. Kimerling, and W. Greene "Ultra-Compact Si-SiO Microring Resonator Optical Channel Dropping Filters," IEEE Photonics Technology Letters, Vol. 10, No. 4, April 1998, pp. 549-551. 


\section{Capítulo 7 - ORR de 2 puertos: Conexión en cascada}

\subsection{Introducción}

Como se explicó en los capítulos anteriores, el ancho de banda de los resonadores ópticos en anillo (ORR) es inversamente proporcional al valor pico del retardo de grupo que producen. Esta característica representa un inconveniente si se desea trabajar con señales de banda ancha. En esta sección se presenta una posible forma de conexión o topología que emplea varios ORRs para sintetizar retardos aproximadamente planos en anchos de banda mayores a los observados en las secciones anteriores. Esta configuración consiste en varias etapas individuales e independientes, como la de la Fig. 7.1, conectadas en serie o cascada. En la Ec. (7.1) se repite la función de transferencia del dispositivo, presentada en el Capítulo 2, mientras que en la Fig.7.2 se muestra el retardo de grupo normalizado que produce en función de la frecuencia angular normalizada con respecto al Free Spectral Range (FSR).

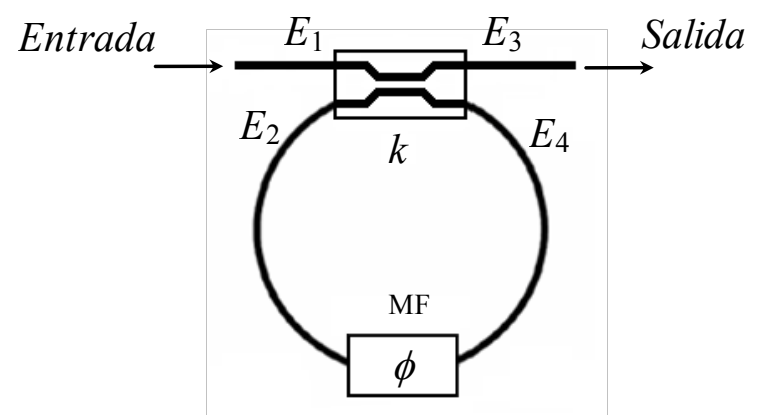

Figura 7.1: Esquema de un ORR controlable. 


$$
t_{31}(\Omega)=\frac{E_{3}}{E_{1}}=\left[\frac{\xi \sqrt{1-k}-\xi^{2} \sqrt{a} \cdot e^{-j \phi} e^{-j \Omega}}{\left(1-\xi \sqrt{1-k} \cdot \sqrt{a} \cdot e^{-j \phi} e^{-j \Omega}\right)}\right]
$$

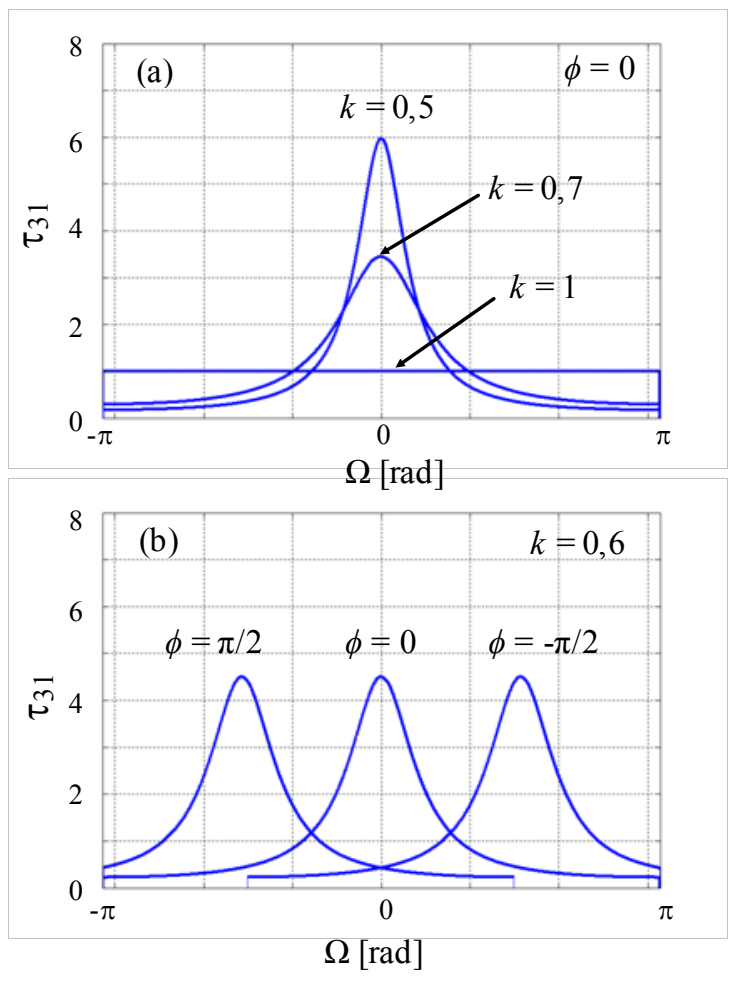

Figura 7.2: Retardo normalizado: (a) Parámetro $k$; (b) Parámetro $\phi$.

\subsection{Conexión en cascada}

Para implementar en forma numérica la conexión en cascada de $\mathrm{N}$ anillos resonadores $\mathrm{y}$ así conformar una línea de retardo, se procede conectando la salida de cada etapa de un único elemento, representada por la Ec. (7.1), con la entrada de la siguiente.

Como los anillos de dos etapas consecutivas no se encuentran acoplados entre sí, las etapas resultan independientes. De esta manera, la función de transferencia del sistema se obtiene como el producto de las funciones de transferencia individuales (Ec. (7.1)), y el retardo de grupo normalizado total como la suma de los retardos individuales. En particular, si $\mathrm{N}$ es el número de anillos idénticos (con los mismos parámetros constructivos) conectados en cascada, las Ecs. (7.2) y (7.3) describen la función de transferencia y el retardo de grupo normalizado del sistema, respectivamente. 


$$
\begin{array}{r}
t(\Omega)=\prod_{i=1}^{N}\left[\frac{\xi \cdot \sqrt{1-k_{i}}-\xi^{2} \cdot \sqrt{a} \cdot e^{-j \phi_{i}} \cdot e^{-j \Omega}}{\left(1-\xi \cdot \sqrt{1-k_{i}} \cdot \sqrt{a} \cdot e^{-j \phi_{i}} \cdot e^{-j \Omega}\right)}\right] \\
\tau(\Omega)=\sum_{i=1}^{N}\left[\frac{\xi^{4} a-\xi^{3} \sqrt{a} \cdot \sqrt{1-k_{i}} \cos \left(\phi_{i}+\Omega\right)}{\xi^{2}\left(1-k_{i}\right)-2 \xi^{3} \sqrt{1-k_{i}} \sqrt{a} \cos \left(\phi_{i}+\Omega\right)+\xi^{4} a}\right. \\
\left.+\frac{\xi \sqrt{1-k_{i}} \sqrt{a} \cos \left(\phi_{i}+\Omega\right)-\xi^{2}\left(1-k_{i}\right) a}{1-2 \xi \sqrt{1-k_{i}} \sqrt{a} \cos \left(\phi_{i}+\Omega\right)+\xi^{2}\left(1-k_{i}\right) a}\right]
\end{array}
$$

En la Fig. 7.3 se muestra, a modo de ejemplo, este tipo de conexión para tres ORRs, yel retardo de grupo normalizado resultante en función de la frecuencia normalizada, $\Omega$.
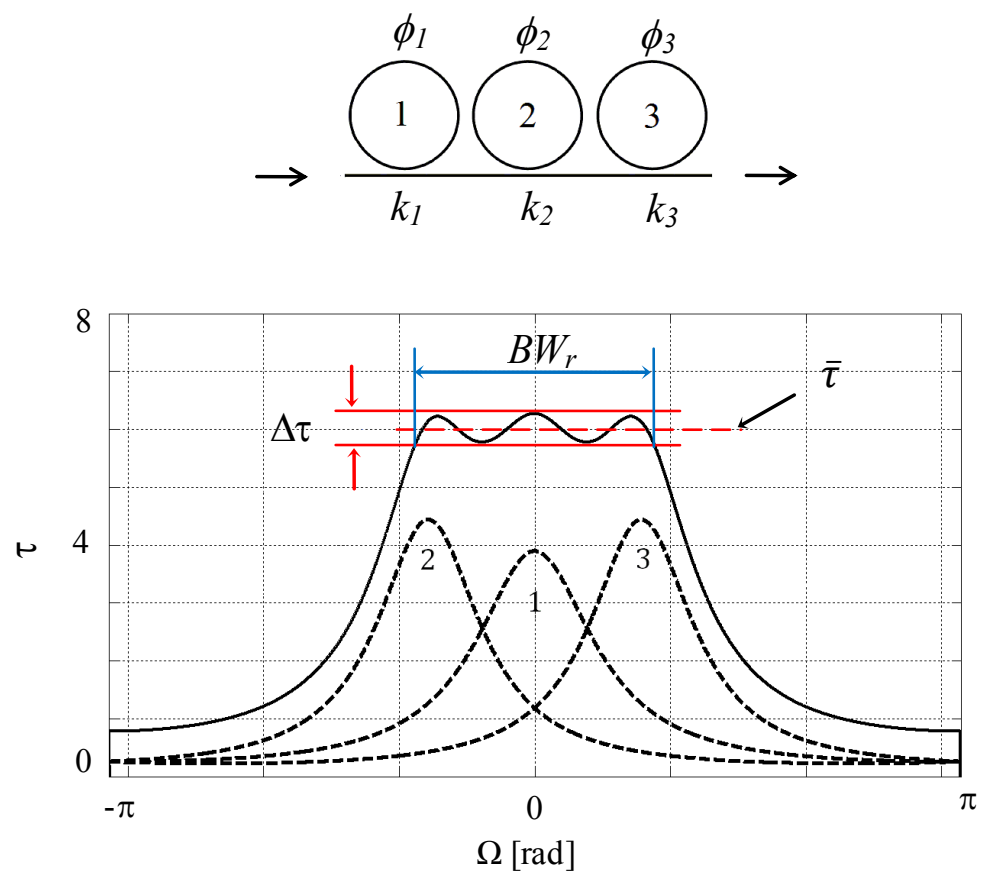

Figura 7.3: Conexión en cascada de tres ORRs controlables.

En la misma figura se indican las características de la respuesta de mayor interés para el presente trabajo. Las variaciones u oscilaciones (pico a pico) que presenta el retardo de grupo normalizado dentro de la banda de interés constituyen lo que se denomina ripple, $\Delta \tau$ del retardo. El ancho de banda de ripple, $B W_{r}$, está definido como el rango de frecuencias donde existe dicho ripple, y el retardo medio, $\bar{\tau}$, es el valor promedio del retardo de grupo 
normalizado dentro de $B W_{r}$. A continuación se analizará el comportamiento del ripple en función del retardo medio que se desea implementar.

\subsection{Análisis}

Como se dijo antes, el objetivo de utilizar este tipo de conexión es sintetizar retardos con respuesta aproximadamente plana en un gran ancho de banda, por lo tanto interesa conocer el comportamiento del ripple al implementar distintos valores de retardo medio. Con el fin de analizar estas pequeñas variaciones, se realizaron simulaciones numéricas en diferentes condiciones de funcionamiento.

Se consideró un sistema compuesto por cinco ORRs con las mismas características que en los capítulos previos: guías de onda de dióxido de silicio $\left(\mathrm{SiO}_{2}\right)$ y nitruro de silicio $\left(\mathrm{Si}_{3} \mathrm{~N}_{4}\right)$, con un índice de refracción efectivo $n_{e f}=1,48$, y pérdidas $\alpha=10 \mathrm{~dB} / \mathrm{m}$. El radio de los anillos, igual a $6 \mathrm{~mm}$, permite determinar, para cada uno, un FSR $=5,38 \mathrm{GHz}$ y $T_{R T}=185,98$ ps. Las pérd idas del acoplador se eligieron nuevamente de valor $\mathrm{P}_{\mathrm{E}}=0,1 \mathrm{~dB}$. Un esquema del sistema propuesto se muestra en la Fig. 7.4.

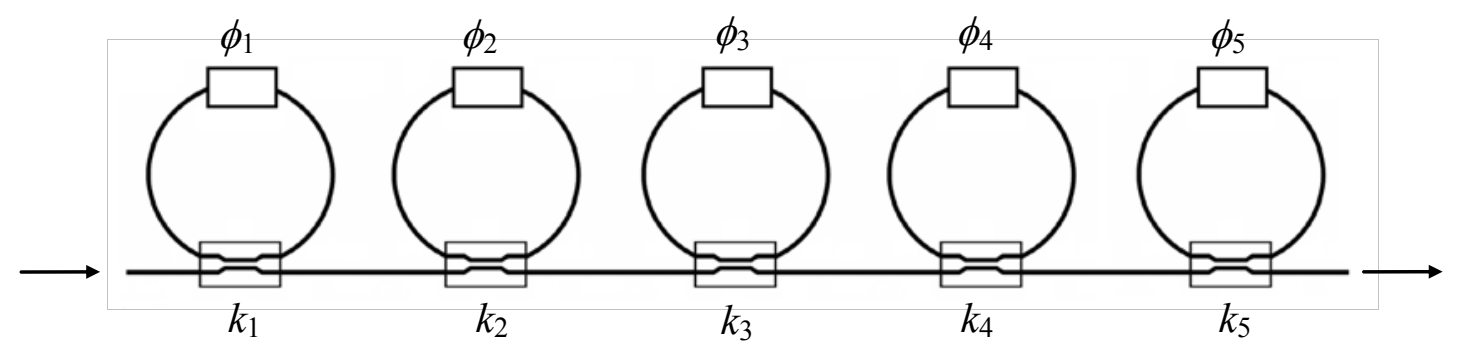

Figura 7.4: Esquema del sistema de cinco ORRs propuesto.

Para reducir los grados de libertad del sistema se fijaron los desplazamientos de fase de los ORRs con los valores: $\phi_{1}=0 ; \phi_{2}=0,2 \pi ; \phi_{3}=-0,2 \pi ; \phi_{4}=0,4 \pi$ y $\phi_{5}=-0,4 \pi$. Luego, el control del retardo medio se realizó mediante la elección adecuada de los factores de acoplamiento de cada anillo, $k_{1}, k_{2}, k_{3}, k_{4} \mathrm{y} k_{5}$.

Para realizar las simulaciones numéricas se utilizó el modelo matemático obtenido en las secciones anteriores para cada etapa de un anillo, y se implementó la conexión en cascada realizando el producto de las funciones de transferencia. Luego, el retardo de grupo se obtuvo como la derivada cambiada de signo de la fase con respecto a la frecuencia. En cada 
caso, se ajustaron los factores de acoplamiento para obtener un determinado retardo medio y luego se corrigieron en forma iterativa sus valores para reducir el ripple lo más posible.

En la Fig. 7.5 se muestra cualitativamente la respuesta de la línea de retardo para cuatro valores distintos de retardo medio: $7 ; 8 ; 8,5$ y 9 .
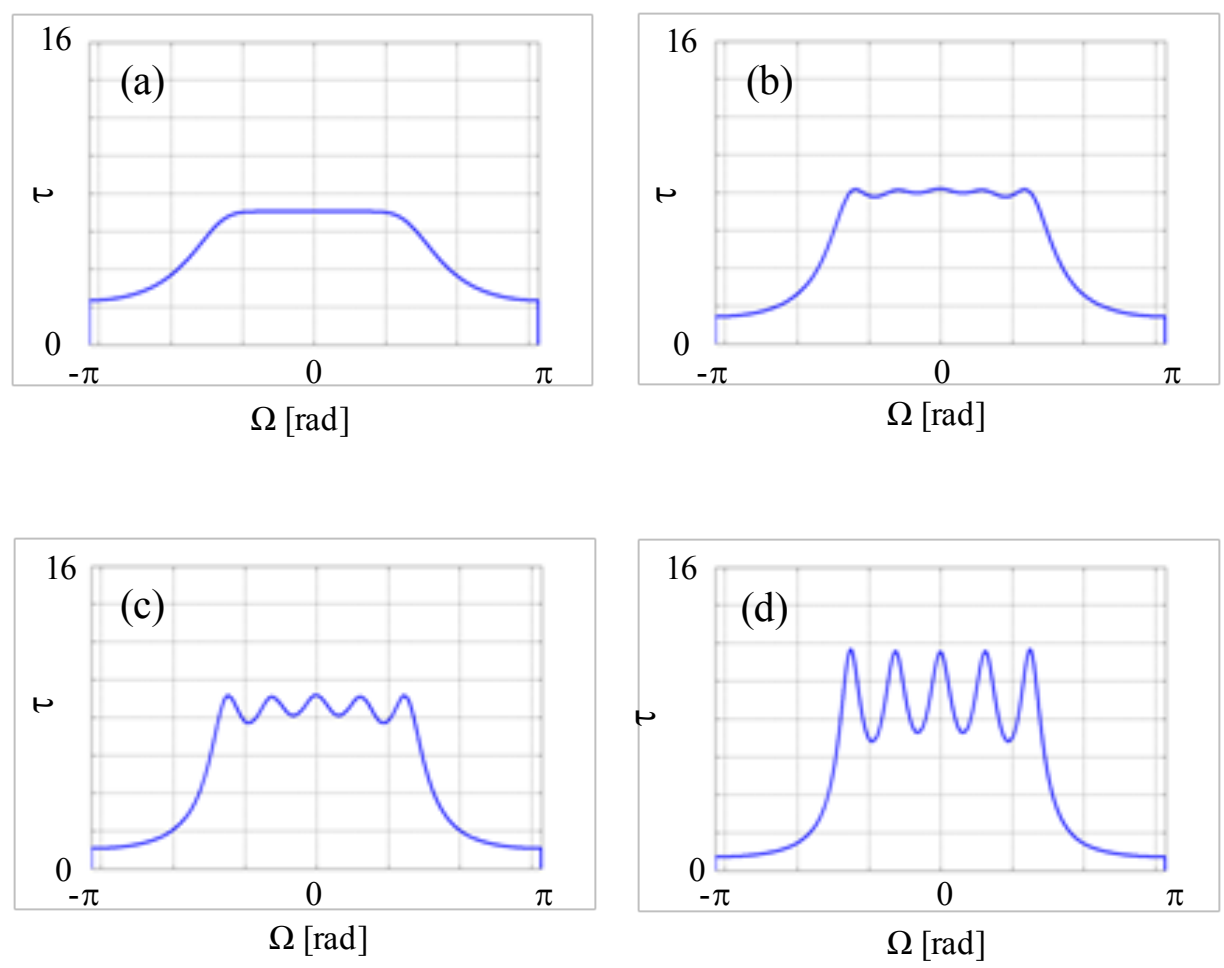

Figura 7.5: Ripple para distintos valores de retardo medio normalizado: (a) 7; (b) 8; (c) 8,5 y (d) 9 .

Como se verificó en los capítulos anteriores, la forma de la curva del retardo de grupo que produce un ORR se hace más angosta al aumentar su valor pico. Debido a esto, al fijar los desplazamientos de fase, los valles entre los retardos individuales que componen la respuesta total del sistema se hacen más pronunciados a medida que aumenta el retardo medio, dando lugar al ripple que se observa en la Fig. 7.5.

En Fig. 7.6 se grafica el valor pico a pico del ripple, $\Delta \tau$, en función del retardo medio, $\bar{\tau}$. Allí se observa que el aumento del ripple es lento al principio, para retardos medios entre $5,5 \mathrm{~T}_{\mathrm{RT}}$ y $8 \mathrm{~T}_{\mathrm{RT}}$, y más rápido para retardos mayores. 


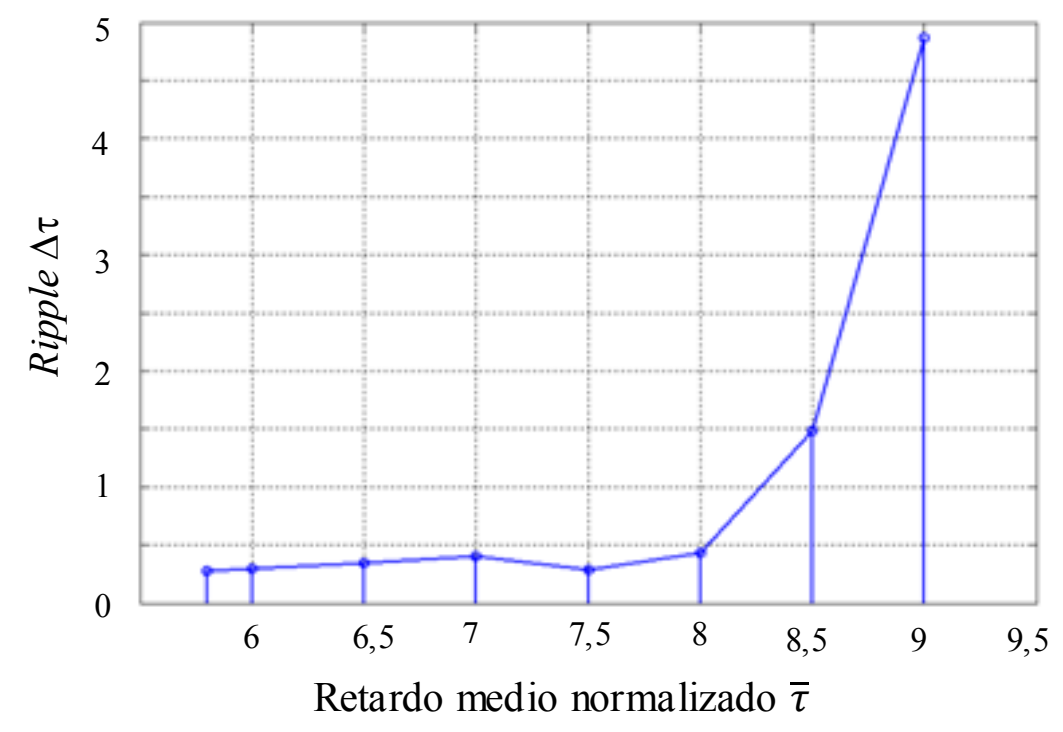

Figura 7.6: Variación del ripple con el retardo medio.

En particular, el valor de $\Delta \tau$ se mantiene entre 0,3 y $0,5 \mathrm{~T}_{\mathrm{RT}}$ ( 55,8 y $93,0 \mathrm{ps}$ en este caso) para retardos medios menores a $8 \mathrm{~T}_{\mathrm{RT}}(1487,8 \mathrm{ps})$, y luego comienza a crecer rápidamente hasta casi $5 \mathrm{~T}_{\mathrm{RT}}(929,9 \mathrm{ps})$ para $\bar{\tau}=9 \mathrm{~T}_{\mathrm{RT}}(1673,8 \mathrm{ps})$. El valor máximo tolerable para el ripple depende de la aplicación particular que se desea desarrollar y en algún caso puede definir un límite superior al retardo medio que el sistema puede implementar cumpliendo con los requerimientos.

Por otro lado, el menor retardo que se puede obtener con anillos en cascada está limitado, ya que cuando los factores de acoplamiento de todos los anillos son iguales a 1, los retardos individuales son de $1 \mathrm{~T}_{\mathrm{RT}}$ para todas las frecuencias, como se muestra en la Fig. 2.7 (a). En este caso, el menor retardo que puede implementarse idealmente es de $5 \mathrm{~T}_{\mathrm{RT}}$.

A partir de la Fig. 7.5, puede intuirse que si se permite modificar la modulación de fase de los anillos que componen la línea de retardo, es posible reducir el ripple del retardo. Esta solución trae aparejada una leve reducción del ancho de banda del sistema, pero aun así puede resultar práctica, como se verá en el Capítulo 9. 


\subsection{Conclusiones}

Con el fin de sintetizar un retardo de grupo con valor medio y ancho de banda determinados, se propuso la conexión en cascada de varias unidades adecuadamente sintonizadas. De esta manera se obtiene una respuesta tal que todas las componentes de frecuencia de una dada señal resultan retardadas aproximadamente en igual cantidad.

El uso de desplazamientos de fase fijos para cada unidad permitió reducir los grados de libertad del problema, de manera de simplificar la síntesis de un determinado retardo. Del análisis, se desprende que para un esquema como el propuesto, el ripple crece con el valor medio retardo generado. Esto se debe a que las respuestas individuales se hacen más angostas a medida que aumenta su valor pico. Si se admite complejizar el control del sistema, y con una leve reducción del ancho de banda, se pueden reducir las variaciones del retardo sintonizando los ORRs que componen el sistema de forma que las frecuencias de resonancia individuales se aproximen cuando el retardo medio aumenta y se alejen cuando este disminuye. De esta manera se pueden lograr retardos de mayor valor con ripple aceptable, extendiendo así el rango útil de retardos que se pueden implementar con el sistema.

Con respecto a la reducción del ancho de banda, el problema puede atenuarse si se contempla la posibilidad de utilizar esquemas de modulación que no exijan tanto al sistema, como por ejemplo un esquema de modulación de banda lateral única (BLU). Otra solución posible consiste en aumentar el número de anillos, aunque a costa de aumentar proporcionalmente la complejidad del control de los retardos. De cualquier manera, la elección de una u otra solución dependerá de los requerimientos y recursos disponibles de la aplicación en cuestión. En el Capítulo 9 se presentará una estrategia que permite, para un sistema de cinco anillos como el analizado, determinar los valores de los factores de acoplamiento y los desplazamientos de fase necesarios para implementar un dado retardo medio con ripple y ancho de banda aceptables. 
Heinrich Sebastian Rabal - Líneas de Retardo Real Para Conformación Óptica de Haces de Microondas 


\section{Capítulo 8 - ORR: conexión en paralelo}

\subsection{Introducción}

En este capítulo se analiza la conexión de resonadores ópticos en anillo (ORR) conocida como ring lattice (que puede traducirse como "enrejado de anillos"), aunque aquí se la denominará "conexión en paralelo" en contraposición a la conexión en cascada, que puede pensarse similar a la conexión en serie de componentes electrónicos.

El objetivo es comparar las características de la respuesta que ofrece esta alternativa con las obtenidas mediante la conexión de anillos en cascada estudiada en el Capítulo 7. En este caso los dispositivos básicos que forman el sistema se encuentran acoplados entre sí, de manera que la luz que ingresa a través del puerto de entrada se acopla sucesivamente a todos los anillos y distintas fracciones de la misma resuenan en cada uno de ellos antes de acoplarse al puerto de salida. Como consecuencia de esto, las distintas etapas del sistema, cada una constituida por un anillo, no son independientes y por lo tanto la respuesta total resultante no puede intuirse a partir de las respuestas individuales de cada anillo.

\subsection{Descripción del sistema}

Como en el caso de un ORR de cuatro puertos, para acoplar la luz entre anillos sucesivos se utiliza un acoplador variable dentro de cada uno. En este caso, la segunda guía de ondas a la cual la luz se acopla no es recta sino con forma de anillo cerrado, constituyendo de esta manera una segunda etapa. Este procedimiento puede extenderse a $\mathrm{N}$ etapas, formando así una red de anillos acoplados, como se muestra en la Fig. 8.1 para el caso de $\mathrm{N}=3$. En la misma figura se indican las señales que se propagan hacia la derecha $\left(F_{0}, . ., F_{\mathrm{N}}\right)$ y hacia la 
izquierda $\left(R_{0}, \ldots, R_{\mathrm{N}}\right)$, así como los campos eléctricos en las entradas, $E_{\mathrm{i} 1}$ y $E_{\mathrm{i} 2}$, y en las salidas, $E_{\mathrm{o} 1} \mathrm{y} E_{\mathrm{o} 2}$.

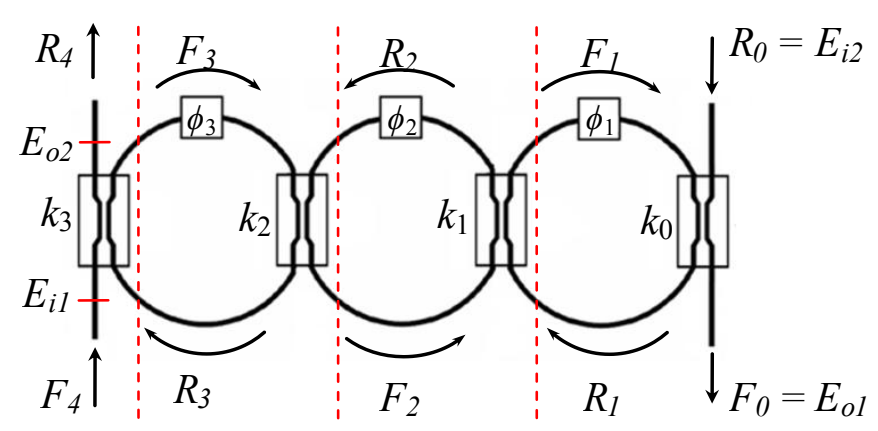

Figura 8.1: Esquema de una red de anillos acoplados $\operatorname{con} \mathrm{N}=3$.

Como se mencionó antes, la respuesta total del sistema no se obtiene como el producto de las respuestas individuales de cada anillo, como si estuvieran en cascada, pero sí se puede recurrir a un método matricial que permite determinar primero una matriz de transferencia para cada etapa simple, y luego extender el algoritmo al agregar más etapas (anillos). En la siguiente sección se muestra el procedimiento y se presentan las funciones de transferencia que relacionan las entradas con las salidas del sistema.

\subsection{Modelo matemático}

Para obtener un modelo matemático que describa la respuesta de una red de anillos como la de la Fig. 8.1, en [1] se presenta un método matricial que permite obtener la función de transferencia de todo el sistema compuesto por etapas individuales o subsistemas. En la Fig. 8.1, las etapas del sistema se encuentran separadas por líneas de puntos, y en la Fig. 8.2 se reproduce sólo una de ellas para analizarla con mayor claridad. Como se observa, cada etapa está constituida por un acoplador y un anillo que puede pensarse dividido en dos mitades con sus características (pérdidas, retardo y fase ad icional) igualmente repartidas. 

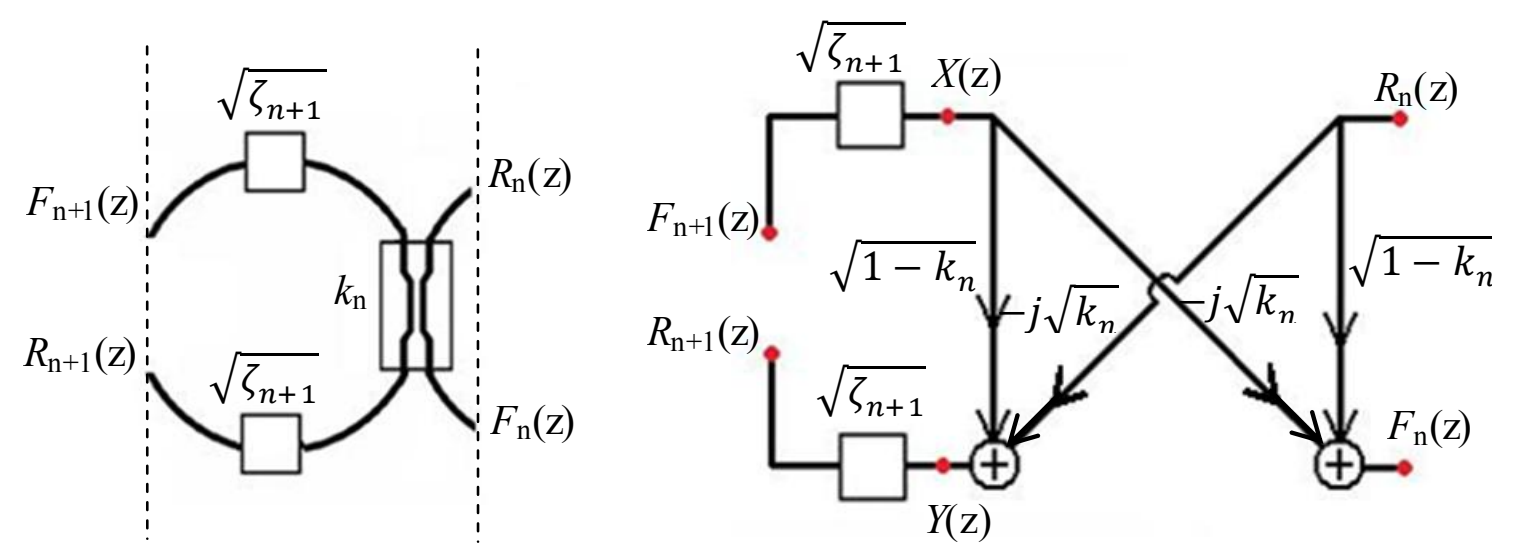

Figura 8.2: Diagrama esquemático de una sola etapa de anillos en paralelo.

De esta manera, si cada etapa como la de la Fig. 8.2 está caracterizada por una matriz de transferencia, la matriz total del sistema se obtiene como el producto matricial de las $\mathrm{N}$ etapas individuales.

En primer lugar, es necesario determinar la matriz de transferencia de una etapa. Si se definen $X(z)$ e $Y(z)$ como los campos eléctricos en los extremos del acoplador conectados al anillo, como se indica en la Fig. 8.2, se pueden determinar la matriz de transferencia del acoplador por separado (Ec. (8.1)), y la de los tramos de guía que forman el anillo (Ec. (8.2)).

$$
\begin{gathered}
{\left[\begin{array}{l}
X(z) \\
Y(z)
\end{array}\right]=\frac{1}{j \sqrt{k_{n}}}\left[\begin{array}{cc}
-1 & \sqrt{1-k_{n}} \\
\sqrt{1-k_{n}} & 1
\end{array}\right]\left[\begin{array}{l}
F_{n}(z) \\
R_{n}(z)
\end{array}\right]} \\
{\left[\begin{array}{l}
F_{n+1}(z) \\
R_{n+1}(z)
\end{array}\right]=\left[\begin{array}{cc}
\frac{1}{\sqrt{\zeta_{n+1}}} & 0 \\
0 & \sqrt{\zeta_{n+1}}
\end{array}\right]\left[\begin{array}{l}
X(z) \\
Y(z)
\end{array}\right]}
\end{gathered}
$$

Luego, la matriz de transferencia de la enésima etapa, $\Phi_{n}$ con $n=0 ; 1 ; 2 \ldots \mathrm{N}$, se obtiene como el producto de las dos matrices anteriores, y después de algunas operaciones algebraicas se llega a:

$$
\left[\begin{array}{l}
F_{n+1}(z) \\
R_{n+1}(z)
\end{array}\right]=\Phi_{n}\left[\begin{array}{l}
F_{n}(z) \\
R_{n}(z)
\end{array}\right]
$$




$$
\Phi_{n}=\frac{1}{-j \sqrt{k_{n}}} \frac{1}{\sqrt{\zeta_{n+1}}}\left[\begin{array}{cc}
1 & -\sqrt{1-k_{n}} \\
-\sqrt{1-k_{n}} \zeta_{n+1} & -\zeta_{n+1}
\end{array}\right]
$$

Donde $\zeta_{n}=\sqrt{a} e^{-j \phi_{n}} Z^{-1}$

Una vez caracterizada cada etapa, la matriz total de un sistema compuesto por $\mathrm{N}$ etapas se obtiene al realizar el producto de las matrices parciales, $\Phi_{T O T}=\Phi_{N} \Phi_{N-1} \ldots \Phi_{0}$, y resulta:

$$
\Phi_{N 0}=\frac{1}{\prod_{0}^{N}\left(-j \sqrt{k_{n}} \sqrt{\zeta_{n+1}}\right)}\left[\begin{array}{cc}
A_{N}(z) & B_{N}^{R}(z) \\
\zeta_{N+1} B_{N}(z) & \zeta_{N+1} A_{N}^{R}(z)
\end{array}\right]
$$

Como resultado del producto matricial, se generan $A_{N}(z)$ y $B_{N}(z)$, que son polinomios en la variable $z$, y en función de los que se definen los polinomios inversos como:

$A_{N}^{R}(z)=(-1)^{N-1} Z^{-N} e^{-j \phi_{t o t}} A_{N}^{*}(z)^{*-1}$

$B_{N}^{R}(z)=(-1)^{N-1} z^{-N} e^{-j \phi_{t o t}} B_{N}^{*}(z)^{*-1}$

De esta manera pueden establecerse dos funciones de transferencia: $t_{11}(z)$ si el puerto de salida es el Puerto 1, y $t_{21}(z)$ si el puerto de salida es el Puerto 2:

$$
\begin{gathered}
t_{11}(z)=\frac{E_{o 1}}{E_{i 1}}=\frac{F_{0}(z)}{\sqrt{\zeta_{N+1}} F_{N+1}(z)}=\frac{(-j)^{N+1} \sqrt{\sigma_{N} e^{-j \phi_{t o t}} \sqrt{a}^{N} z^{-N}}}{A_{N}(z)} \\
t_{21}(z)=\frac{E_{o 2}}{E_{i 1}}=\frac{R_{N+1}(z)}{\zeta_{N+1} F_{N+1}(z)}=\frac{B_{N}(z)}{A_{N}(z)}
\end{gathered}
$$

Donde:

$\sigma_{N}=\prod_{0}^{N} k_{n}$

$\phi_{t o t}=\phi_{1}+\phi_{2}+\cdots+\phi_{N}$

En cada caso, una vez definidos los parámetros constructivos del sistema y la cantidad de etapas, se determinan los polinomios $A_{N}(z)$ y $B_{N}(z)$, y con ellos las funciones de transferencia. 


\subsection{Análisis de la respuesta}

A priori, resulta intuitivo pensar que en esta configuración la selectividad de la respuesta aumente, debido a que, a medida que la luz se acopla a los sucesivos anillos, debe satisfacer más condiciones de resonancia que para un anillo simple. En ese sentido, cada anillo que se incorpora a la red modifica la condición de resonancia del anterior debido a que introduce una fase dependiente de la frecuencia en la señal, como se muestra en la descripción de la respuesta de un ORR simple, en la Fig. 2.6(b). Esto explica la aparición de nuevos picos de resonancia al aumentar la cantidad de anillos acoplados, como se observa en los siguientes gráficos, donde se presentan el coeficiente de transmisión de intensidad y el retardo de grupo en función de la frecuencia.

Debido a la gran cantidad de variables y grados de libertad del sistema, se decidió realizar simulaciones generales en algunas situaciones específicas que son ilustrativas, permitiendo analizar la performance y caracterizar la transmisión y el retardo de grupo de la red de anillos. Además, mediante simulaciones previas se encontró que el efecto de la modulación de fase, $\phi$, es complejo de predecir y no aporta mejoras significativas a la forma de la respuesta en cuanto a la generación de retardos uniformes de banda ancha. Por lo tanto, para el análisis que se presenta a continuación, todos los desplazamientos de fase fueron considerados iguales a cero.

Para las simulaciones numéricas se distinguieron dos casos: en el primero se analiza la función de transferencia $t_{21}(\Omega)$, cuando no hay acoplamiento a una segunda guía al final de la red $\left(k_{0}=0\right)$ y por lo tanto el sistema se comporta como una red de dos puertos. En el segundo caso, el acoplamiento a una segunda guía recta se realiza a través del acoplador $k_{0}$ en el último anillo $\left(k_{0} \neq 0\right)$ y el sistema actúa como una red de cuatro puertos con dos salidas cuyas funciones transferencias con respecto a la entrada 1 son $t_{21}(\Omega)$ y $t_{11}(\Omega)$. De estas dos funciones, sólo se analiza $t_{11}(\Omega)$ debido a que $t_{21}(\Omega)$ produce resultados similares a los del caso anterior.

Se consideraron las mismas características, como dimensiones, materiales, etc. que para las simulaciones previas, y se analizaron tres condiciones de operación diferentes: $(i)$ todos los acoplamientos de igual valor; (ii) el valor de los acoplamientos se incrementa con cada anillo añadido; y (iii) el valor de los acoplamientos se reduce con cada anillo añadido. En 
todos los casos, los gráficos muestran la variación de la respuesta al incrementar/reducir todos los factores de acoplamiento en pasos de 0,1 .

Sin embargo, los resultados correspondientes a la situación en que el factor de acoplamiento de cada anillo que se agrega a la red es mayor que el del anterior no se presentan debido a que:

- Para una red de dos puertos, la respuesta del sistema en dicho caso resultó muy similar a la obtenida en el primero, cuando todos los factores son iguales, salvo por algunas diferencias en los valores pico de retardo.

- Para una red de cuatro puertos, la respuesta es idéntica a la que se obtiene para el caso de factores de acoplamiento decrecientes.

Con esta salvedad, a continuación se presentan los resultados obtenidos al simular el sistema en sus dos configuraciones posibles, y en las condiciones de operación mencionadas.

\section{Red de dos puertos $\left(k_{0}=0\right)$}

En las Figs. 8.3(a) y (b) se muestran el coeficiente de transmisión de intensidad y el retardo de grupo que se obtienen a partir de la función de transferencia $t_{21}(\Omega)$ para la condición en que todos los factores de acoplamiento son iguales, es decir $k_{3}=k_{2}=k_{1}$. Con líneas rojas se indica el caso $\left[k_{3} k_{2} k_{1}\right]=[0,10,10,1]$, y con líneas azules el caso $\left[k_{3} k_{2} k_{1}\right]=[0,70,70,7]$. Adicionalmente, en las Figs. 8.3(c) y (d) se presentan las mismas curvas para la condición de operación en que el factor de acoplamiento de cada anillo añadido es menor que el del anillo previo, es decir $k_{3}>k_{2}>k_{1}$, en una cantidad de 0.1. En este caso, las líneas rojas corresponden a los factores $\left[k_{3} k_{2} k_{1}\right]=[0,30,20,1]$, mientras que las líneas azules a los factores $\left[k_{3} k_{2} k_{1}\right]=[0,90,80,7]$. 

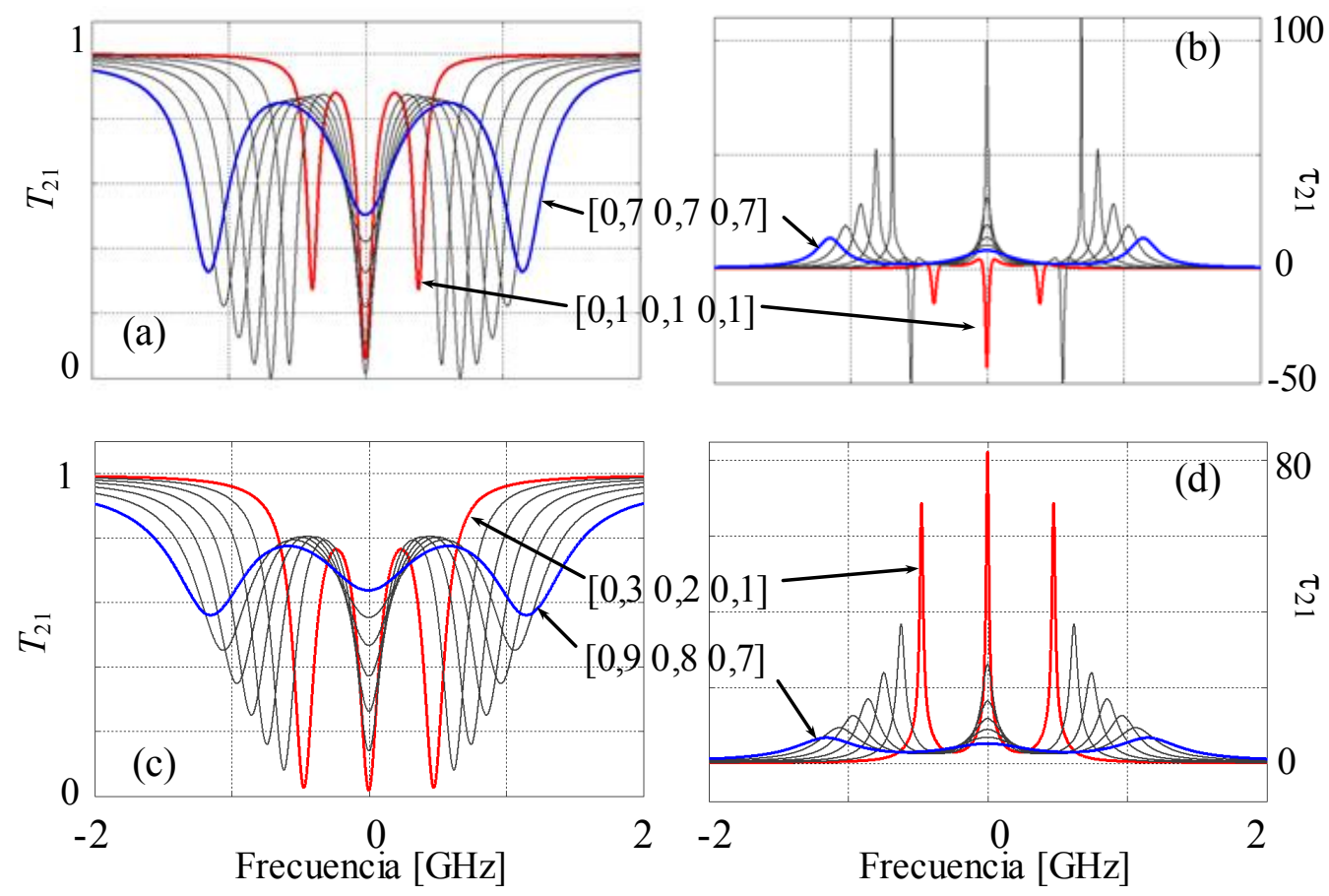

Figura 8.3: Coeficiente de transmisión de intensidad y retardo de grupo: (a) y (b) Factores de acoplamiento iguales; (c) y (d) Factores de acoplamiento crecientes.

Como se observa en las figuras, el número de picos de resonancia coincide con el número de anillos acoplados, al igual que en la configuración en cascada, pero con la diferencia de que en este caso todos los desplazamientos de fase son cero, y por lo tanto la ubicación de estos picos no es sencilla de predecir dado que depende de muchas variables.

En los dos casos presentados, el coeficiente de transmisión de intensidad exhibe valles (o picos de atenuación) que se vuelven menos selectivos y se separan unos de otros a medida que se incrementan los factores de acoplamiento. Sin embargo, las amplitudes relativas de estos valles son diferentes en ambos casos. Cuando todos los factores de acoplamiento tienen valores iguales, la diferencia entre las amplitudes del valle central y los valles externos es mayor que para el caso de factores decrecientes. Además, en el segundo caso los tres picos de atenuación alcanzan valores cercanos a 0 cuando los factores de acoplamiento toman sus valores mínimos. 
Por otro lado, el retardo de grupo exhibe picos muy agudos y selectivos que reducen su amplitud y también se separan en frecuencia a medida que aumentan los factores de acoplamiento.

En la Fig. 8.3(b), aunque no se observa debido a que el gráfico está cortado por razones de claridad, los picos de retardo alcanzan valores de hasta 393,0 $\mathrm{T}_{\mathrm{RT}}$, con FWHM aproximado de $5 \mathrm{MHz}$. En la misma figura se observa que existe una condición dentro de los valores simulados en la que el retardo de grupo presenta valores negativos. En particular, esto ocurre para los valores más pequeños de los factores de acoplamiento. A medida que estos aumentan, el retardo se hace positivo y alcanza su máximo valor. Luego los picos se reducen y se separan hasta la condición indicada con color azul, en la que el valor máximo de retardo es de 13,4 $\mathrm{T}_{\mathrm{RT}}$ y las variaciones dentro del ancho de banda son de aproximadamente $11,6 \mathrm{~T}_{\mathrm{RT}}$.

En la Fig. 8.3(d), para el caso de factores decrecientes, puede verse que el retardo alcanza valores de hasta 82,3 $\mathrm{T}_{\mathrm{RT}}$, en el pico central, y $68,7 \mathrm{~T}_{\mathrm{RT}}$ en los picos laterales. Estos picos se reducen, se ensanchan y se separan a medida que el valor de los factores de acoplamiento se incrementa. La última condición simulada muestra una respuesta ensanchada, con un retardo medio de 4,8 $\mathrm{T}_{\mathrm{RT}}$ y variaciones de $\pm 1,9 \mathrm{~T}_{\mathrm{RT}}$.

Esta configuración, en las condiciones analizadas, no resulta adecuada para la síntesis de retardos de gran ancho de banda. Los picos del retardo de grupo son demasiado selectivos cuando se implementan retardos grandes, y aunque se ensanchan al reducir el valor pico, las variaciones resultantes dentro del ancho de banda son igualmente importantes. Además, distintas simulaciones del sistema revelaron una gran dificultad para predecir el comportamiento de estos picos, y por lo tanto, para implementar una estrategia de control que permita minimizar las variaciones del retardo de grupo.

\section{Red de cuatro puertos $\left(k_{0} \neq 0\right)$}

Como se mencionó antes, en este caso se analiza la función de transferencia $t_{11}(\Omega)$, hacia la salida de la segunda guía de onda, por lo tanto la luz presente a la salida es una fracción de la que resuena en los anillos. 


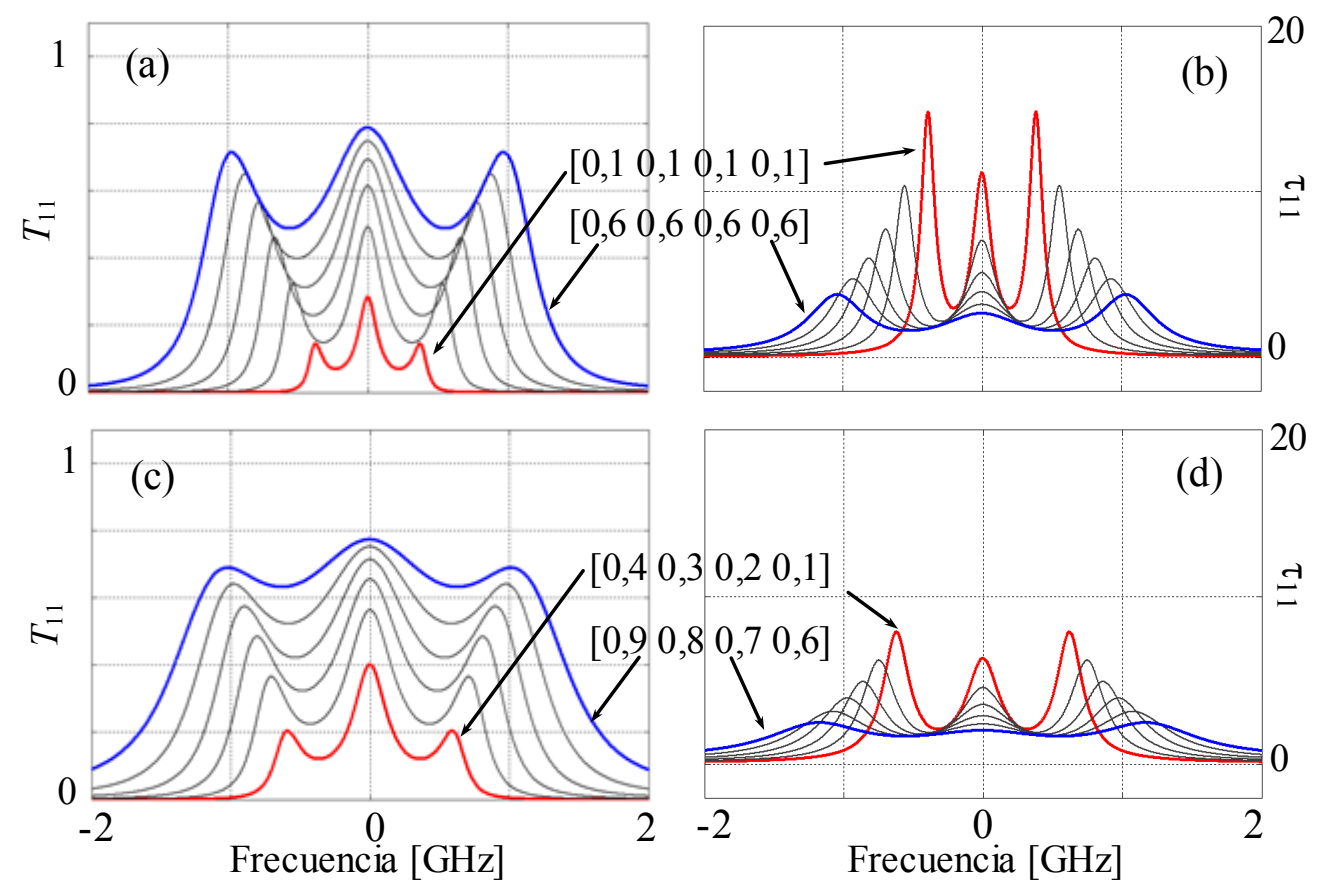

Figura 8.4: Coeficiente de transmisión de intensidad y retardo de grupo: (a) y (b) Factores de acoplamiento iguales; (c) y (d) Factores de acoplamiento crecientes.

En las Figs. 8.4(a) y (b) se muestran el coeficiente de transmisión de intensidad y el retardo de grupo para la condición en que los factores de acoplamiento son iguales. Las líneas rojas corresponden al caso $\left[k_{3} k_{2} k_{1} k_{0}\right]=[0,10,10,10,1]$, y las líneas azules el caso $\left[k_{3} k_{2} k_{1} k_{0}\right]=[0,60,60,60,6]$.

Como puede verse, el coeficiente de transmisión de intensidad exhibe tres picos, de los cuales el central es el mayor. La altura de estos picos aumenta al incrementar los factores de acoplamiento, al mismo tiempo que se separan y se hacen más anchos. Esto tiende a reducir las variaciones o ripple que toman un valor de aproximadamente 0,3 .

Con respecto al retardo, se observa que los picos alcanzan valores menores que en la red de dos puertos: 11,2 $\mathrm{T}_{\mathrm{RT}}$ el pico central y 14,8 $\mathrm{T}_{\mathrm{RT}}$ los dos picos laterales. Además, estos picos son más anchos, con FWHM entre 0,13 y $0,14 \mathrm{GHz}$. Al incrementar los factores de acoplamiento, la altura de los picos se reduce y aumenta la separación entre ellos, ensanchando la respuesta total, que en el último caso (indicado con azul) está caracterizada por un retardo medio de 2,7 $\mathrm{T}_{\mathrm{RT}}$ con variaciones de $\pm 1,1 \mathrm{~T}_{\mathrm{RT}}$ en un rango de frecuencias de alrededor de 2,72 GHz. 
Por último, en las Figs. 8.4(c) y (d) se presentan los gráficos correspondientes a la condición de factores de acoplamiento decrecientes: en rojo, el caso $\left[k_{3} k_{2} k_{1} k_{0}\right]=[0,40,3$

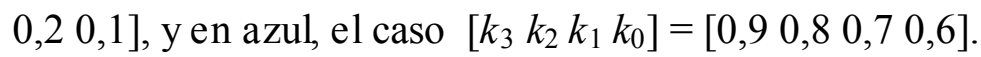

La respuesta es bastante similar al caso anterior, aunque con picos menos pronunciados. Debido a esto, tanto el coeficiente de transmisión de intensidad como el retardo resultan levemente más uniformes que en el caso anterior. En particular, el pico central y los picos laterales del retardo alcanzan los valores máximos de 6,3 y 7,9 $\mathrm{T}_{\mathrm{RT}}$, respectivamente, para el rango de acoplamientos simulado. El retardo más uniforme se alcanza para el último caso, indicado con azul, donde la respuesta tiene un retardo medio de 2,1 $\mathrm{T}_{\mathrm{RT}}$ con variaciones de $\pm 0,4 \mathrm{~T}_{\mathrm{RT}}$ en un rango de frecuencias de alrededor de 3,04 GHz. Aunque estas variaciones relativamente pequeñas en un gran ancho de banda son características deseables para el sistema, no debe perderse de vista que esto se cumple en una condición para la cual el retardo medio es muy pequeño comparado con el que puede obtenerse mediante la conexión en cascada (entre 10 y $20 \mathrm{~T}_{\mathrm{RT}}$ ).

\subsection{Conclusiones}

En este capítulo se demostró que la conexión de ORRs en paralelo permite, en ciertas condiciones, generar retardos relativamente grandes en comparación con la conexión en cascada estudiada en el Capítulo 7. Se analizaron dos configuraciones: una red de dos puertos, y una de cuatro puertos. Debido a que en la estructura de dos puertos la señal debe propagarse desde el puerto de entrada, en el lado izquierdo, hacia lado derecho y luego volver al puerto de salida, nuevamente en el lado izquierdo, el retardo obtenido puede ser mayor que en una estructura de cuatro puertos y una estructura basada en anillos en cascada. En las Figs. 8.3(d) y (b), los retardos obtenidos alcanzan valores mayores a 80 y de hasta 393 veces el tiempo de round-trip, mientras que en las Figs. 8.4(b) y (d) los valores pico son menores a 20 y $10 \mathrm{~T}_{\mathrm{RT}}$, respectivamente. Sin embargo, las variaciones (ripple) dentro del ancho de banda de interés no son despreciables en ninguno de los dos casos, y suponen un inconveniente para sintetizar retardos para señales de banda ancha. Simulaciones adicionales revelaron que el comportamiento de los picos de retardo es bastante complicado y difícil de predecir, por lo que la estrategia de control que se requiere para lograr reducir dichas variaciones es más compleja que para la configuración de anillos 
en cascada descripta en el Capítulo 7. En ese sentido, la conexión en cascada es más efectiva y simple de controlar para obtener retardos cercanos a $10 \mathrm{~T}_{\mathrm{RT}}$ pero con menor variación dentro del ancho de banda.

Cabe mencionar que la topología presentada en este capítulo, aunque no resulte práctica para la implementación de retardos de banda ancha, sí puede serlo para otro tipo de aplicaciones. Por ejemplo aquellas aplicaciones que requieran la implementación de grandes retardos pero muy selectivos, con anchos de banda del orden de $5 \mathrm{MHz}$, o por el contrario, aplicaciones que requieran pequeños retardos, del orden de 372 ps ( $2 \mathrm{~T}_{\mathrm{RT}}$ en este caso), con anchos de banda cercanos a $3 \mathrm{GHz}$.

Con el objetivo implementar de retardos reales de banda ancha que puedan luego ser aplicados en un sistema conformador de haz, se propone la conexión en cascada como la alternativa más apropiada entre las analizadas. A continuación, en elCapítulo 9, se presenta un procedimiento que permite determinar los parámetros de control de un sistema basado en ORRs conectados en cascada con el fin de implementar retardos con determinadas características.

\section{Referencias}

[1] C. K. Madsen, Optical Filter Design and Analysis - A Signal Processing Approach, John Wiley \& Sons, Inc. (1999). 
Heinrich Sebastian Rabal - Líneas de Retardo Real Para Conformación Óptica de Haces de Microondas 


\section{Capítulo 9 - ORR en cascada: algoritmo de control}

\subsection{Introducción}

En capítulos anteriores se estudiaron el funcionamiento y las características de ORRs controlables, y luego de analizar un par de topologías que permiten mejorar ciertas características de la respuesta, se propuso la conexión en cascada de varios de ellos con el fin de aumentar el ancho de banda de retardo del sistema. Como se verificó en el Capítulo 7, para llevar a cabo dicha implementación es necesario calcular los valores de los factores de acoplamiento y desplazamientos de fase de cada anillo de manera que la respuesta total resulte plana en un cierto ancho de banda, salvo por una pequeña variación o ripple.

En esta sección se presenta procedimiento o algoritmo bastante simple que permite determinar los parámetros de control de cada ORR que compone el sistema para lograr una cierta condición de funcionamiento con un error tolerable. 


\subsection{Breve descripción del sistema}

El componente elemental del sistema consiste en un anillo resonador óptico (ORR) como el que se muestra en la Fig. 9.1, donde se indica la amplitud del campo eléctrico, el factor de acoplamiento $k$, y el modulador de fase dentro del anillo, que se utiliza para añadir un desplazamiento de fase, $\phi$.

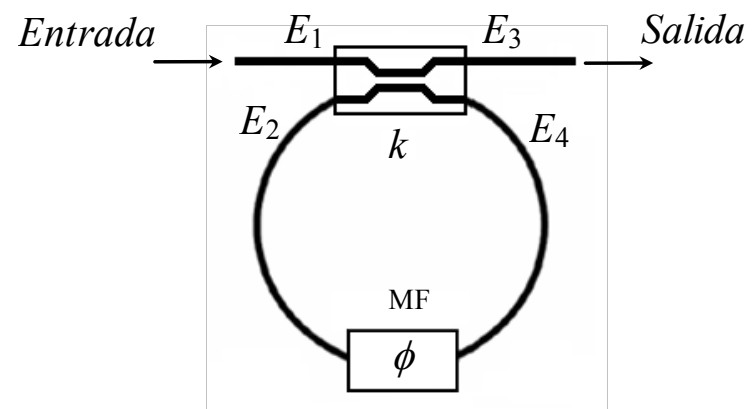

Figura 9.1 : Esquema de un ORR controlable.

La función de transferencia que relaciona los campos eléctricos a la salida y a la entrada, $E_{3}$ y $E_{1}$, fue obtenida a partir de las ecuaciones características de sus componentes y está dada por la Ec. (9.1).

$$
t_{31}(\Omega)=\frac{E_{3}}{E_{1}}=\left[\frac{\xi \sqrt{1-k}-\xi^{2} \sqrt{a} \cdot e^{-j \phi} e^{-j \Omega}}{\left(1-\xi \sqrt{1-k} \cdot \sqrt{a} \cdot e^{-j \phi} e^{-j \Omega}\right)}\right]
$$

Los parámetros $k, \phi, \xi$ y a representan el factor de acoplamiento, el desplazamiento de fase adicional, las pérdidas del acoplador y las pérdidas de la guía de onda que forma el anillo, respectivamente, mientras que $\Omega$ es la frecuencia angular normalizada con respecto al Free Spectral Range.

En el Capítulo 2 se definió el coeficiente de transmisión de intensidad como el módulo de (9.1) elevado al cuadrado y el retardo de grupo como la derivada cambiada de signo de la fase de (9.1) con respecto a la frecuencia.

En función de $\Omega$, y para una dada condición de acoplamiento y pérdidas, el coeficiente de transmisión de intensidad, la fase, y el retardo de grupo exhiben la forma que se muestra en la Fig. 9.2. En la misma figura se indican algunas características de interés, como el FWHM y el valor pico del retardo. 


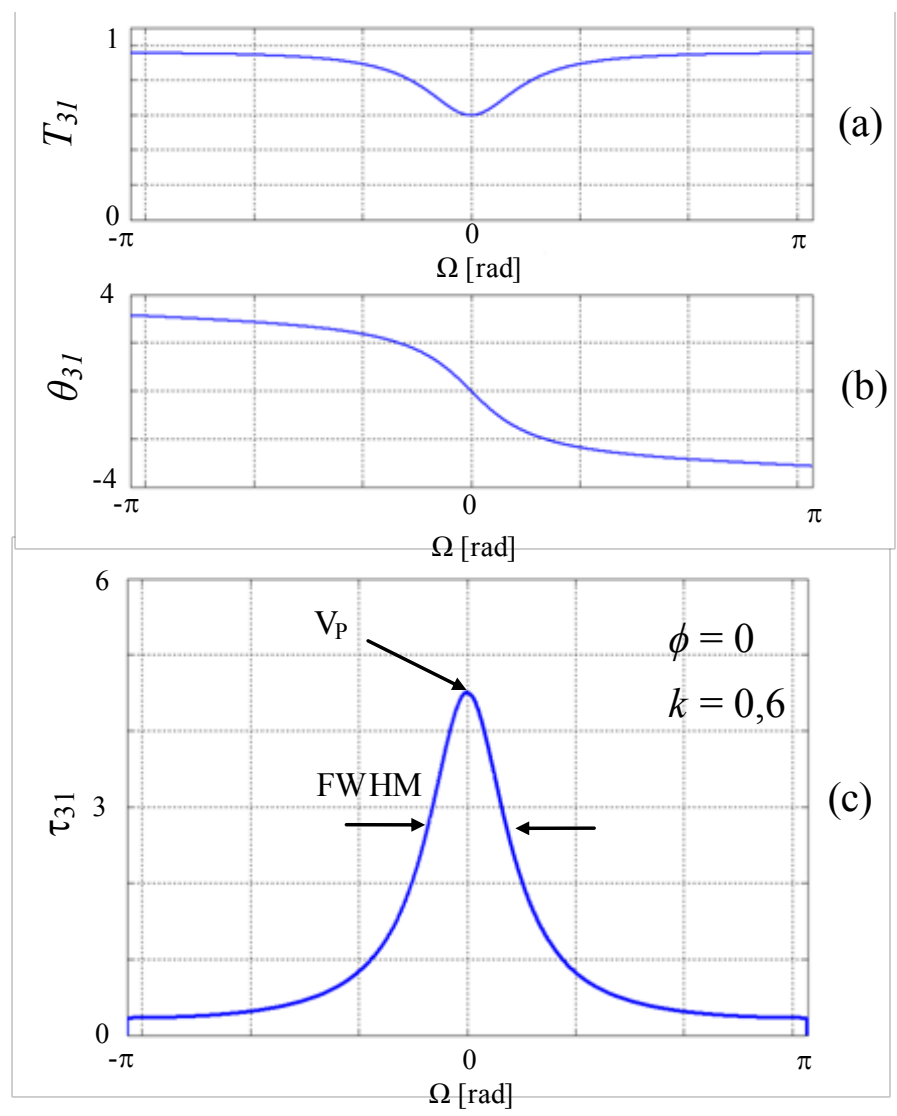

Figura 9.2: Respuesta de un ORR: (a) Transmisión de intensidad; (b) Fase y (c) Retardo de grupo normalizado.

\section{Parámetros de control}

Más allá de que todos los parámetros que intervienen en la Ec. (9.1) influyen de una u otra manera en la respuesta del dispositivo, el factor de acoplamiento, $k$, y el desplazamiento de fase, $\phi$, son los parámetros que permiten modificar el valor pico del retardo y la frecuencia de resonancia, respectivamente, una vez construido (Ver Capítulo 2). En la Fig. 9.3(a) y (b) se muestra la influencia de dichos parámetros en el retardo de grupo. 


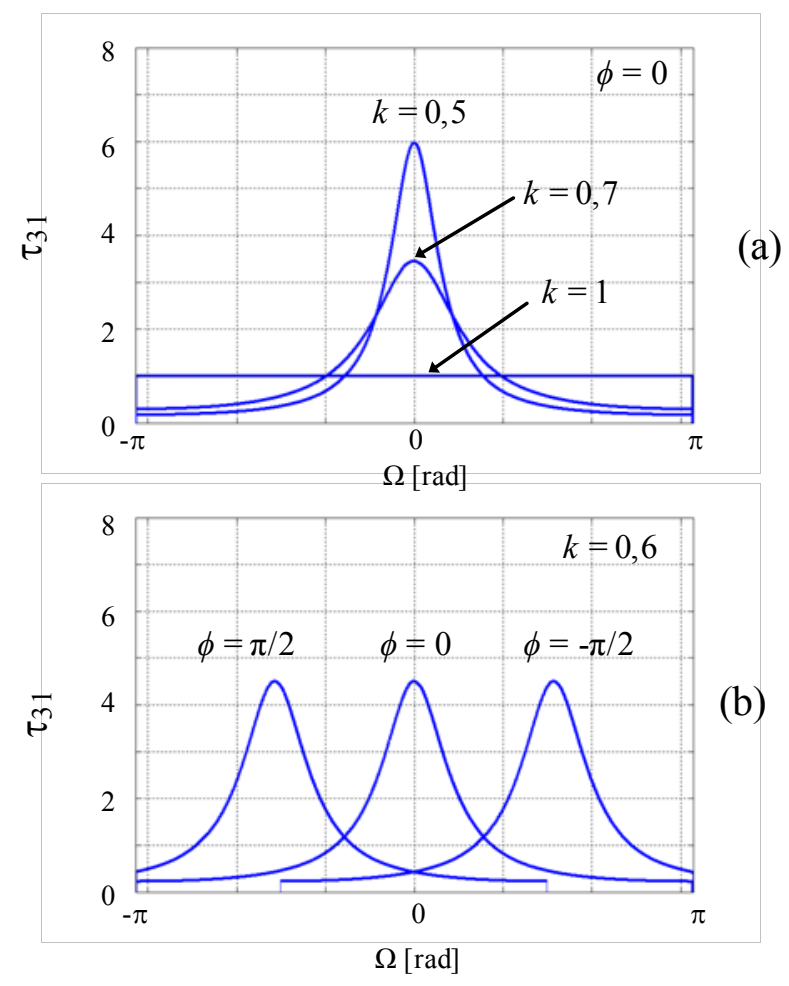

Figura 9.3: Retardo normalizado: (a) Parámetro $k$; (b) Parámetro $\phi$.

\section{Acopla miento crítico}

La condición de acoplamiento crítico, analizada con detalle en el Capítulo 4, determina dos zonas de trabajo: una subacoplada y una sobreacoplada. Debido a que en la primera aparecen retardos de grupo negativos en la respuesta del dispositivo y que la forma de la misma no permite sintetizar retardos uniformes de forma simple, en esta sección se decidió utilizar el dispositivo siempre en la zona de trabajo sobreacoplada, es decir con factores de acoplamiento mayores al factor crítico, $k_{\mathrm{c}}$. 


\section{Conexión en cascada}

En el Capítulo 7 se presentó la conexión en cascada de varios ORRs y se analizó el retardo de grupo total del sistema. En particular se observó la evolución del ripple al aumentar el retardo medio implementado. A modo de ejemplo, en la Fig. 9.4 se repite el gráfico del retardo de grupo normalizado resultante de una cascada de tres ORRs. Se indican, además, las características de interés definidas en los capítulos previos: ripple $\Delta \tau$, retardo medio $\bar{\tau}$, y ancho de banda de ripple $B W_{\mathrm{r}}$
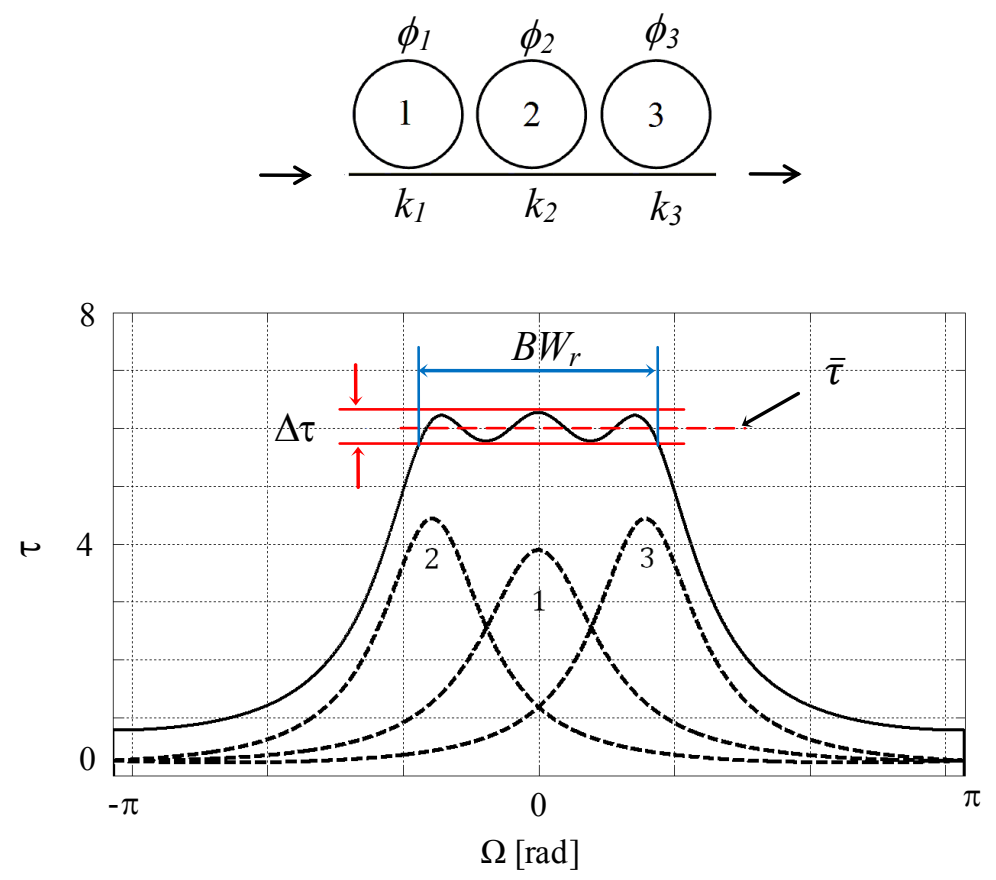

Figura 9.4: Conexión en cascada de tres ORRs controlables.

En este capítulo se propone un procedimiento para determinar los valores de los parámetros de control (factores de acoplamiento y desplazamientos de fase) de una línea de retardo compuesta por $\mathrm{N}$ anillos resonadores en cascada. 


\subsection{Algoritmo de control}

El algoritmo o procedimiento que se presenta en este capítulo tiene por objetivo determinar los parámetros de control de un sistema conformado por $\mathrm{N}$ anillos resonadores conectados en cascada que permitan obtener la respuesta con las características deseadas.

La estrategia que se propone es bastante simple. En forma resumida, consiste en generar tablas de look up con datos obtenidos mediante simulaciones numéricas del sistema en las condiciones de funcionamiento deseadas. Durante las simulaciones, se modifican los parámetros de control del sistema hasta lograr un dado retardo medio con el menor ripple distribuido uniformemente en la banda de interés. Una vez que se logra esta condición, se registran todos los datos de interés, como son retardo medio, el ripple, el ancho de banda y, lo más importante, los valores de los parámetros de control correspondientes. Estos datos se utilizan luego para obtener, por medio de un ajuste, un conjunto de funciones que permitan generar las tablas de look up que el sistema utilizará en tiempo real para sintetizar los retardos.

El diagrama de flujo de la Fig. 9.5 resume los distintos pasos del procedimiento, que luego se describe con más detalle y a través de un ejemplo concreto. 


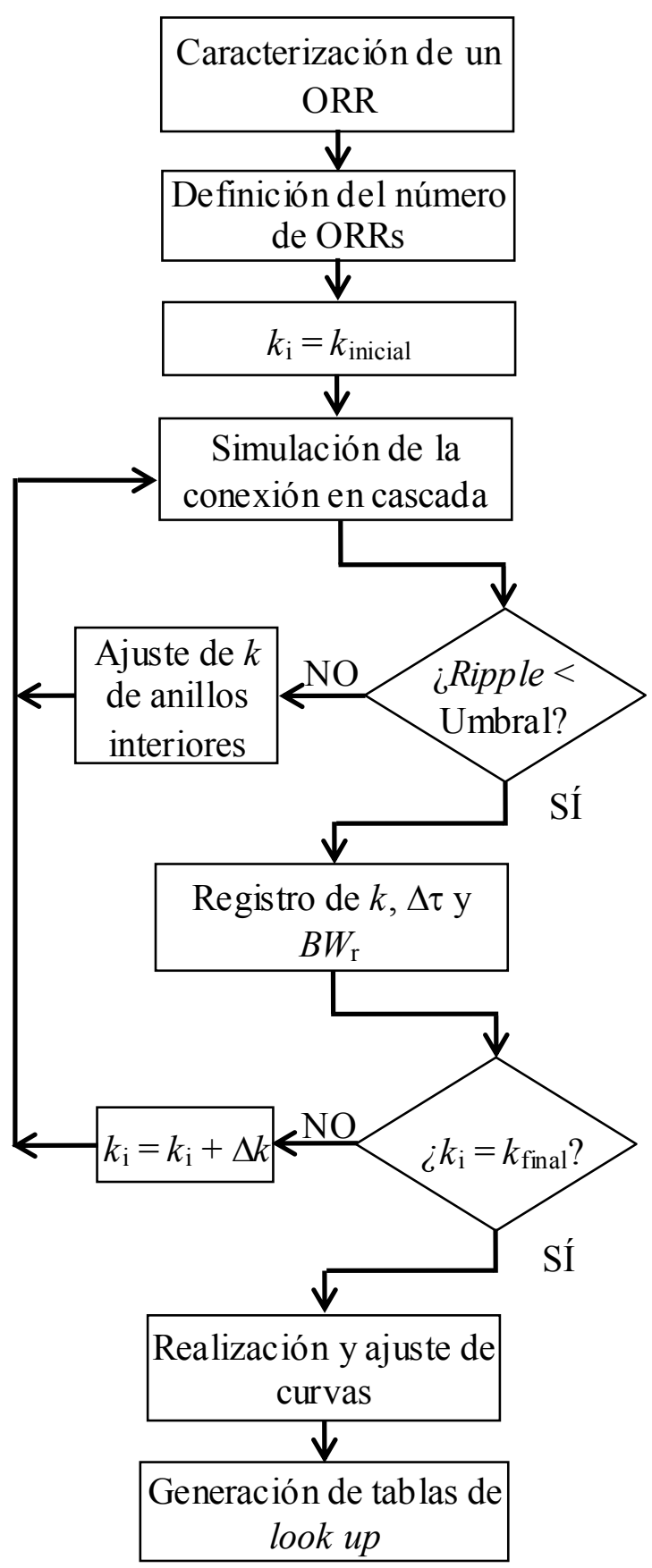

Figura 9.5: Diagrama de flujo del procedimiento propuesto. 


\section{Caracterización de un ORR}

El primer paso del procedimiento propuesto consiste en caracterizar la respuesta de un único anillo, para lo cual se realizan simulaciones numéricas del retardo de grupo en función de la frecuencia angular normalizada utilizando la Ec. (9.2), donde $\theta_{31}(\Omega)$ representa la fase de la transferencia $t_{31}(\Omega)$ presentada en la Ec. (9.1). Debe tenerse en cuenta que algunos parámetros constructivos y de los materiales, como las dimensiones, las pérdidas, índice de refracción, etc. influyen en las características de la respuesta, por lo tanto la caracterización debe hacerse utilizando los valores definitivos del sistema que se desea implementar. Sin embargo, si en el futuro sólo se desea modificar el número de anillos del sistema, manteniendo sus características constructivas, no es necesario realizar esta caracterización nuevamente. Para este ejemplo se adoptaron las mismas características constructivas que en todos los capítulos anteriores.

$$
\tau_{31}(\Omega)=-\frac{d \theta_{31}(\Omega)}{d \Omega}
$$

Como se verificó en el Capítulo 3 y se observa en la Fig. 9.3(b), la fase adicional que aporta el modulador de fase dentro del anillo únicamente actúa como un desplazamiento en frecuencia de la respuesta, sin modificar su forma. Por el contrario, el factor de acoplamiento, $k$, gobierna el valor pico y el FWHM del retardo, sin modificar la frecuencia de resonancia. Por lo tanto, para caracterizar la respuesta del ORR, alcanza con fijar el desplazamiento de fase en 0 rad y efectuar simulaciones numéricas del retardo de grupo para distintas condiciones de acoplamiento. Con ese objetivo, para el presente ejemplo se eligió un rango de variación para $k$ entre 0,2 y 0,9 , con incrementos de 0,05 , y en cada instancia se efectuaron los cálculos del valor pico y el FWHM del retardo de grupo normalizado mediante las ecuaciones que se describen en el Apéndice B. Luego, con los datos obtenidos, se realizaron los gráficos que se muestran en la Fig. 9.6, que describen la variación de los parámetros mencionados en función del factor de acoplamiento. 


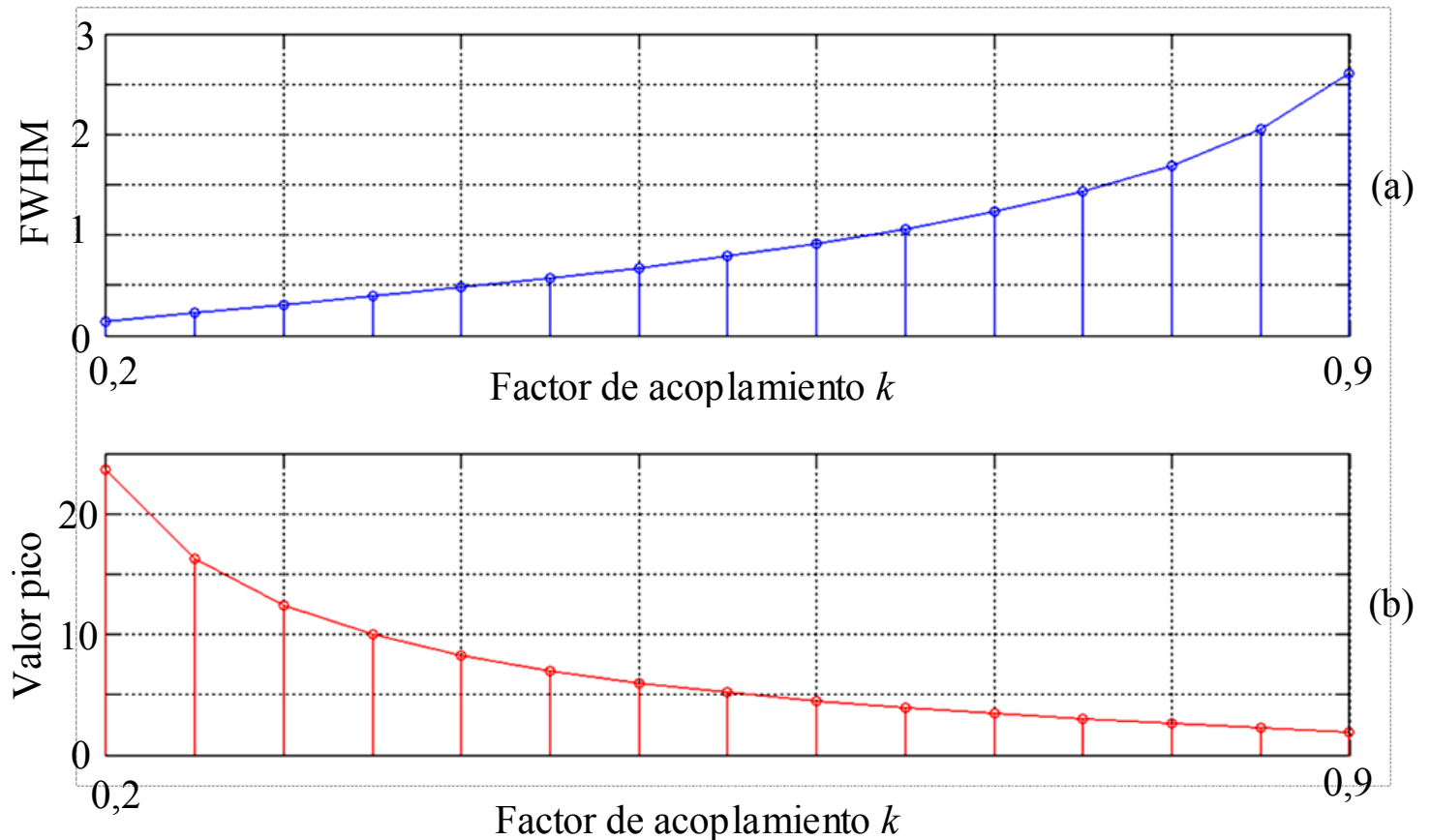

Figura 9.6: (a) FWHM y (b) Valor pico del retardo de grupo en función del factor de acoplamiento.

Se observa que al incrementar el factor de acoplamiento se reduce el valor pico, al mismo tiempo que aumenta el FWHM, indicando que la respuesta se hace más ancha. Es importante tener en cuenta este comportamiento debido a que tiene un efecto directo sobre el ripple del retardo producido por una cascada de ORRs, como se explicó en el Capítulo 7.

\section{Simulación de la conexión en cascada}

El siguiente paso consiste en implementar en forma numérica la conexión en cascada de $\mathrm{N}$ anillos resonadores para conformar una línea de retardo. El número de resonadores a emplear depende del ancho de banda y el retardo máximo requeridos por la aplicación posterior. En el presente análisis se eligió, sin pérdida de generalidad, un número impar de ORRs para simplificar la descripción del procedimiento de diseño, aunque fácilmente puede ser extendido a un número par de elementos. En particular, se decidió implementar un sistema compuesto por cinco resonadores idénticos. 
Debido a la simetría espectral de la respuesta del sistema, si está compuesto por $\mathrm{N}$ anillos, sólo hace falta calcular (N-1)/2 factores de acoplamiento y la misma cantidad de desplazamientos de fase. Para el caso de cinco anillos, se requiere calcular $k_{0}, k_{1}, k_{2}, \phi_{0}, \phi_{1}$ y $\phi_{2}$, como se indica en la Fig. 9.7.

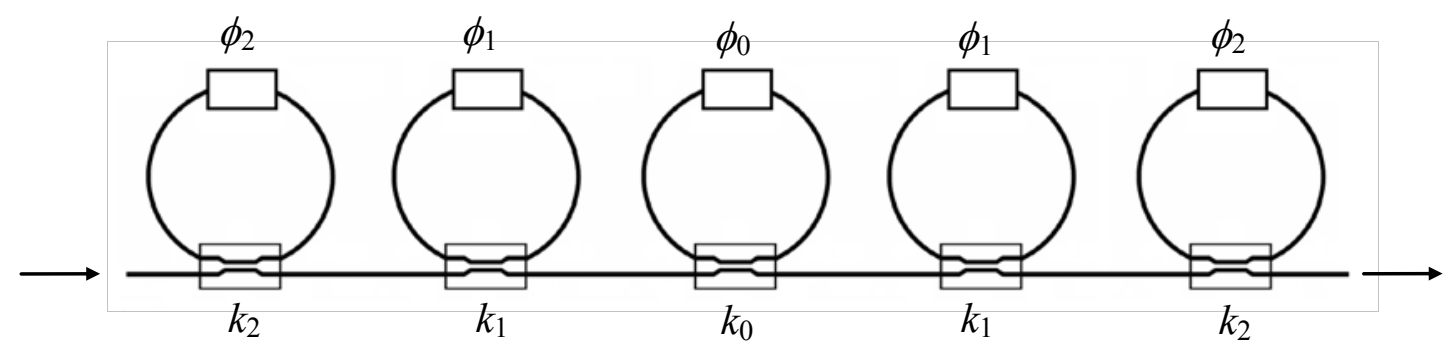

Figura 9.7: Esquema del sistema de cinco ORRs propuesto.

El rango de variación del factor de acoplamiento se eligió, nuevamente, entre los valores 0,2 y 0,9 con incrementos de 0,05 , y cada instancia la simulación se realizó partiendo del sistema en una condición determinada, en la cual:

- El factor de acop lamiento es el mismo para todos los ORRs $\left(k_{0}=k_{1}=k_{2}=k_{\text {inicial }}\right)$

- Uno de los anillos se sintoniza a la frecuencia de resonancia ( $\left.\phi_{0}=0 \mathrm{rad}\right)$ por razones de simplicidad. Para los anillos restantes, ubicados a ambos lados en forma simétrica, se propuso que los desplazamientos de fase de cada ORR se determinen en función del FWHM y de manera que la superposición de las “colas" de cada respuesta ayude a reducir la variación entre los picos. Se adoptó entonces el criterio de que al inicio de la simulación la separación en frecuencia entre las respuestas de dos ORRs adyacentes sea igual a la mitad del FWHM correspondiente al factor de acoplamiento de acuerdo con el gráfico de la Fig. 9.6(a). En la Fig. 9.8 se ejemplifica el criterio adoptado para el caso de dos ORRs. Cabe mencionar que esta condición sólo se ajusta al inicio de cada simulación, y luego los desplazamientos de fase quedan fijos durante la optimización de la respuesta, que se lleva a cabo por medio de los factores de acoplamiento. De esta manera se facilita el control, ya que se reducen los grados de libertad del sistema. 


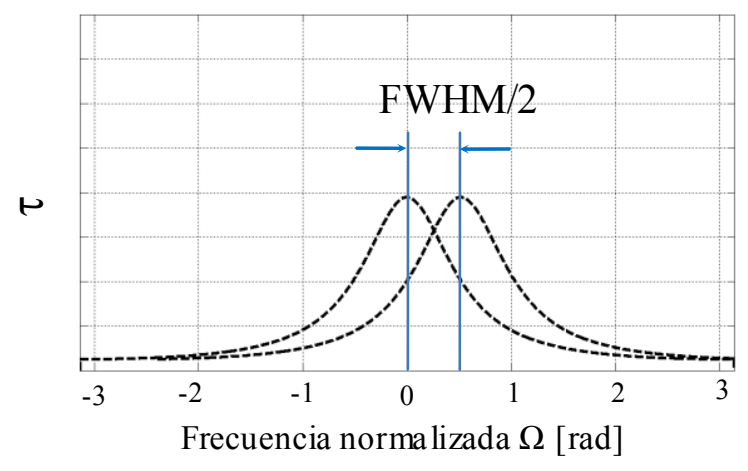

Figura 9.8: Criterio adoptado para la separación entre las respuestas de ORRs adyacentes.

A modo de ejemplo, en la Fig. 9.9 se muestran los retardos producidos por cada uno de los cinco ORRs y el retardo resultante al inicio de una de las simulaciones, en las condiciones iniciales mencionadas. Se observa que todos los anillos aportan un retardo considerable a la frecuencia central de resonancia que se suma al retardo del anillo central, lo que determina la forma de campana resultante. Se hace evidente, por lo tanto, la necesidad de actuar sobre los parámetros de control, que en este caso son los factores de acoplamiento, para reducir las variaciones del retardo dentro de la banda de interés y así obtener una respuesta aproximadamente plana.

Para ello, se propuso reducir los picos de los retardos más cercanos a la frecuencia de resonancia, incluyendo el central, y mantener sin cambios los dos más alejados. Como se desprende del gráfico de la Fig. 9.6(b), para reducir el pico del retardo producido por un ORR se debe incrementar el factor de acoplamiento del mismo. Por lo tanto, se fijó el valor del factor de acoplamiento $k_{2}$ de los anillos externos y se incrementaron los factores de los anillos internos, $k_{0} \mathrm{y} k_{1}$, de manera de reducir los picos de los retardos correspondientes. 


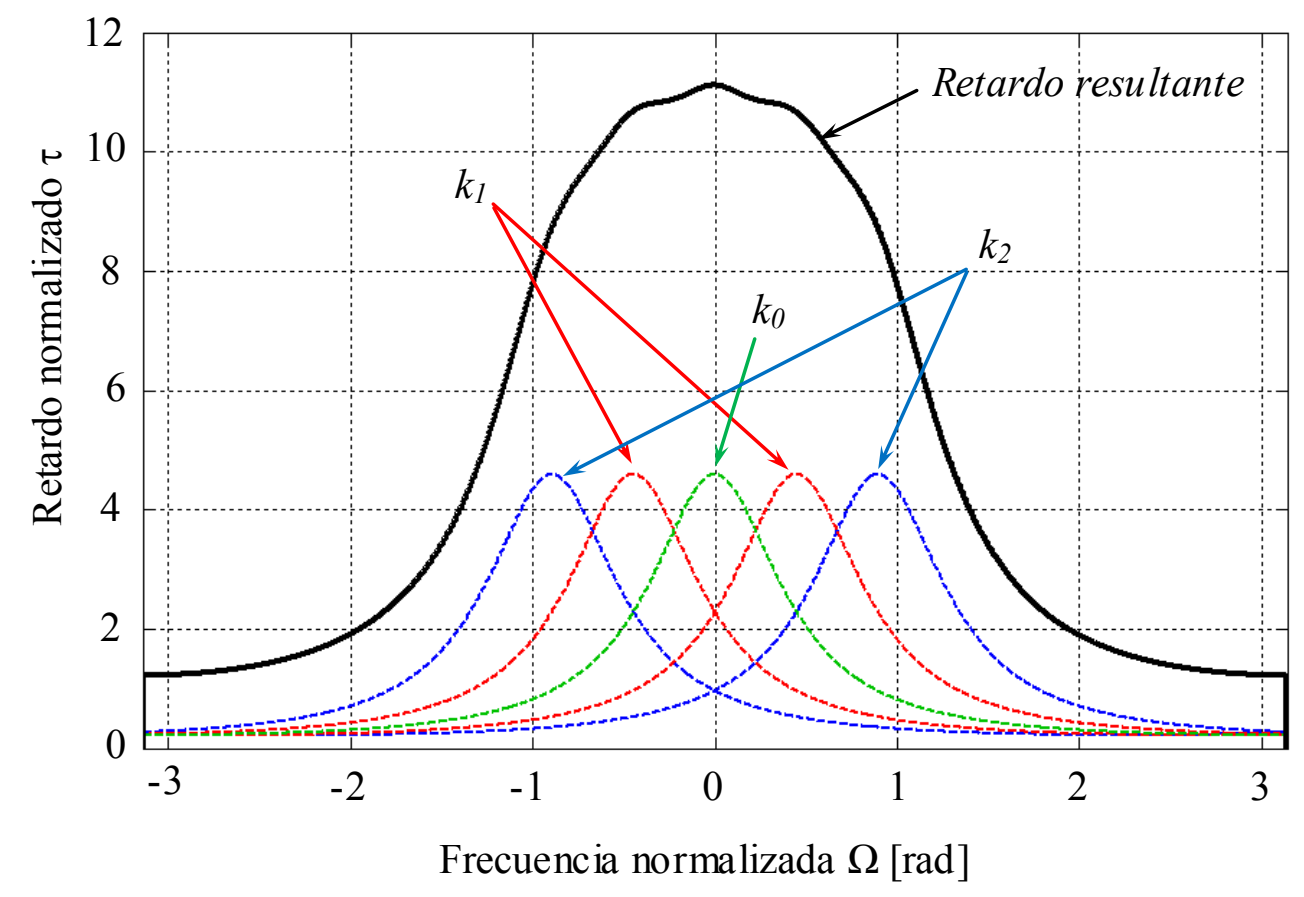

Figura 9.9: Retardo resultante al iniciar la simulación de la cascada de ORRs.

En un proceso iterativo, primero se incrementó el valor de $k_{0}$ y luego el de $k_{1}$, observando luego de cada modificación el estado del ripple. Este proceso finalizó al lograr el menor ripple que cumpla con la condición de tener los picos por encima y por debajo del valor medio de igual amplitud en la banda de interés. En la Fig. 9.10 se muestra la respuesta aproximadamente plana obtenida al finalizar la simulación y una ampliación en la zona de interés que permite apreciar el estado del ripple resultante.

Es necesario mencionar que en cada instancia también se realizaron simulaciones simultáneas del coeficiente de transmisión de intensidad. En las Figs. 9.11 y 9.12 se muestra el estado del coeficiente de transmisión de intensidad resultante al iniciar y al finalizar la simulación, respectivamente. Se observa que exhibe una forma muy similar a la del retardo de grupo, aunque invertida. En la Fig. 9.12 también se muestra una ampliación de la zona de interés, donde puede verse un pequeño ripple en la transmisión de intensidad. 


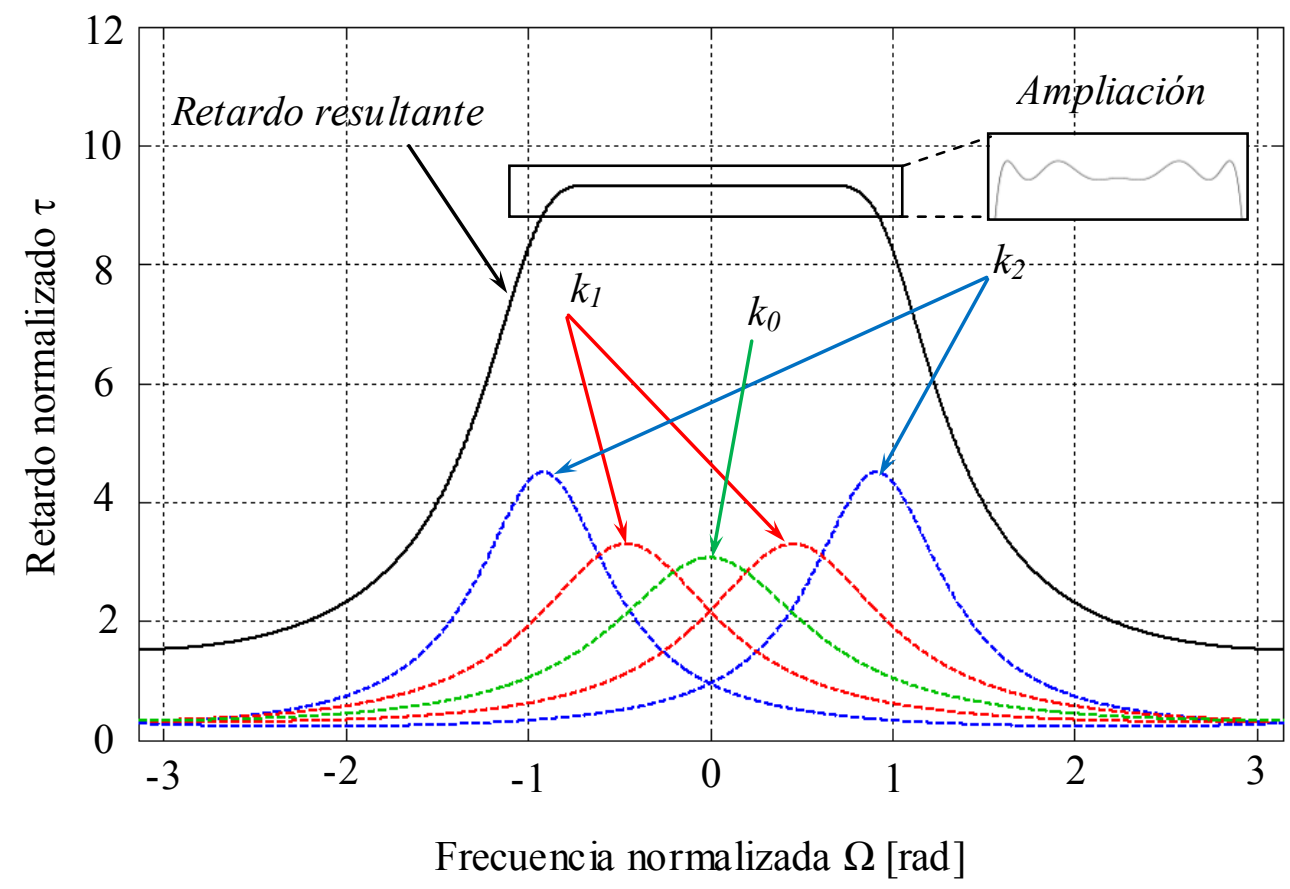

Figura 9.10: Retardo resultante al finalizar la simulación. En la ampliación de la zona de interés puede observarse un pequeño ripple uniforme.

Una vez lograda la condición mencionada para el ripple del retardo, el paso siguiente consiste en registrar los valores de las características de interés: la transmisión de intensidad media, el retardo medio $\bar{\tau}$, el ripple, y el ancho de banda de ripple, $B W_{\mathrm{r}}$. De esta manera finaliza la primera instancia de simulación y a continuación se procede de igual manera para el siguiente valor del factor de acoplamiento del rango de variación adoptado, $k_{\mathrm{i}}+\Delta k$.

El proceso de registro de datos finaliza cuando se registran los correspondientes a $k_{\mathrm{i}}=0,9$, que es el límite superior del rango de factores de acoplamiento. Los datos obtenidos de esta manera se vuelcan en una en una tabla y permiten realizar los gráficos que se muestran en la siguiente sección. 


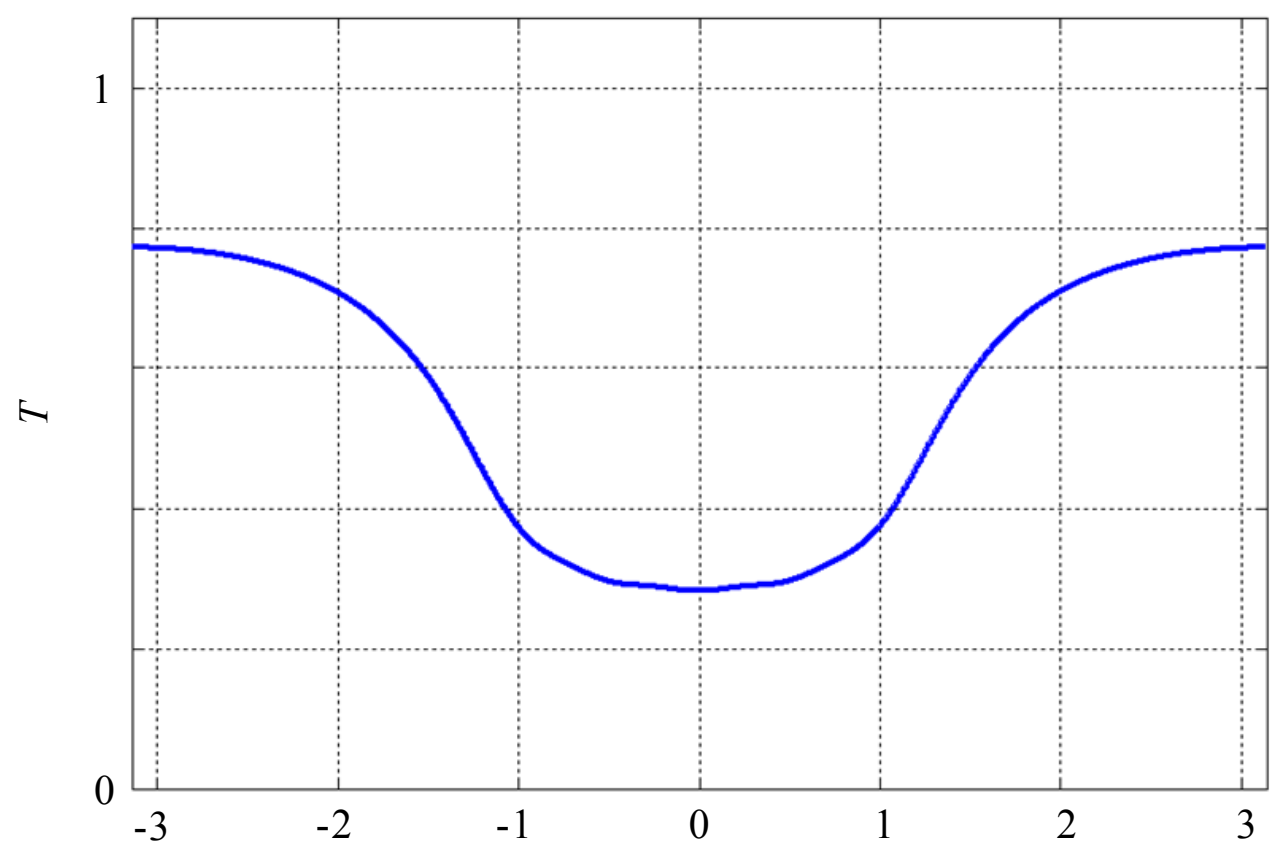

Frecuencia normalizada $\Omega[\mathrm{rad}]$

Figura 9.11: Coeficiente de transmisión de intensidad al iniciar la simulación de la conexión en cascada de ORRs.

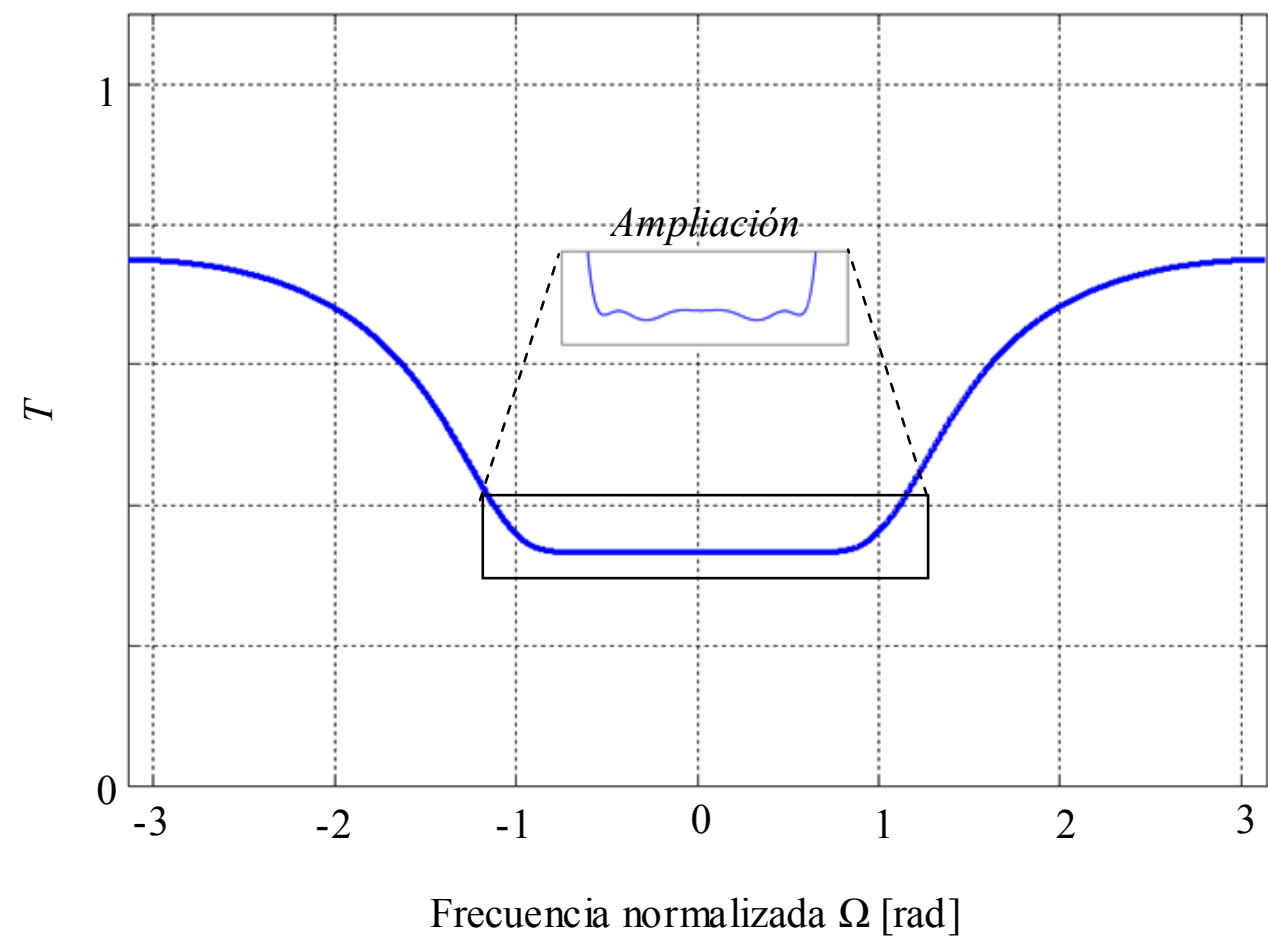

Figura 9.12: Coeficiente de transmisión de intensidad al finalizar la simulación de la conexión en cascada de ORRs. 


\subsection{Presentación gráfica de los datos registrados}

\section{Transmisión de intensidad y rango de trabajo}

Debido a que cada ORR se comporta como un filtro sintonizable cuya atenuación en la banda de interés es inversamente proporcional al factor de acoplamiento, cuando se desean implementar retardos de gran valor, la potencia transmitida a la salida se reduce. Esto limita el rango de retardos posibles ya que, para luego recuperar la señal, el nivel de potencia transmitida debe ser detectable. Un valor razonable para la transmisión de intensidad mínima puede ser de al menos $20 \%$. De esta manera queda determinado un límite superior para el retardo medio que el sistema puede generar, definido como $R_{\text {máx }}$. Por otro lado, también se puede establecer un límite inferior, $R_{\text {min }}$, debido a limitaciones prácticas de implementación, ya que retardos muy pequeños corresponden a factores de acoplamiento mayores a 0,9 , difíciles de lograr.

En la Fig. 9.13 se observa el comportamiento antes mencionado del sistema, que actúa como un filtro en la banda de frecuencias de trabajo y cuya transmisión media disminuye al aumentar el retardo medio. En el gráfico se indica además, sombreado con color verde, el rango de trabajo, que está definido como el rango de retardos medios entre los límites $R_{\text {mín }}$ y $R_{\text {máx }}$. Para el sistema considerado, estos límites son de 5,5 $\mathrm{T}_{\mathrm{RT}}(1,02 \mathrm{~ns})$ y $13,7 \mathrm{~T}_{\mathrm{RT}}$ $(25,5 \mathrm{~ns})$, respectivamente. 


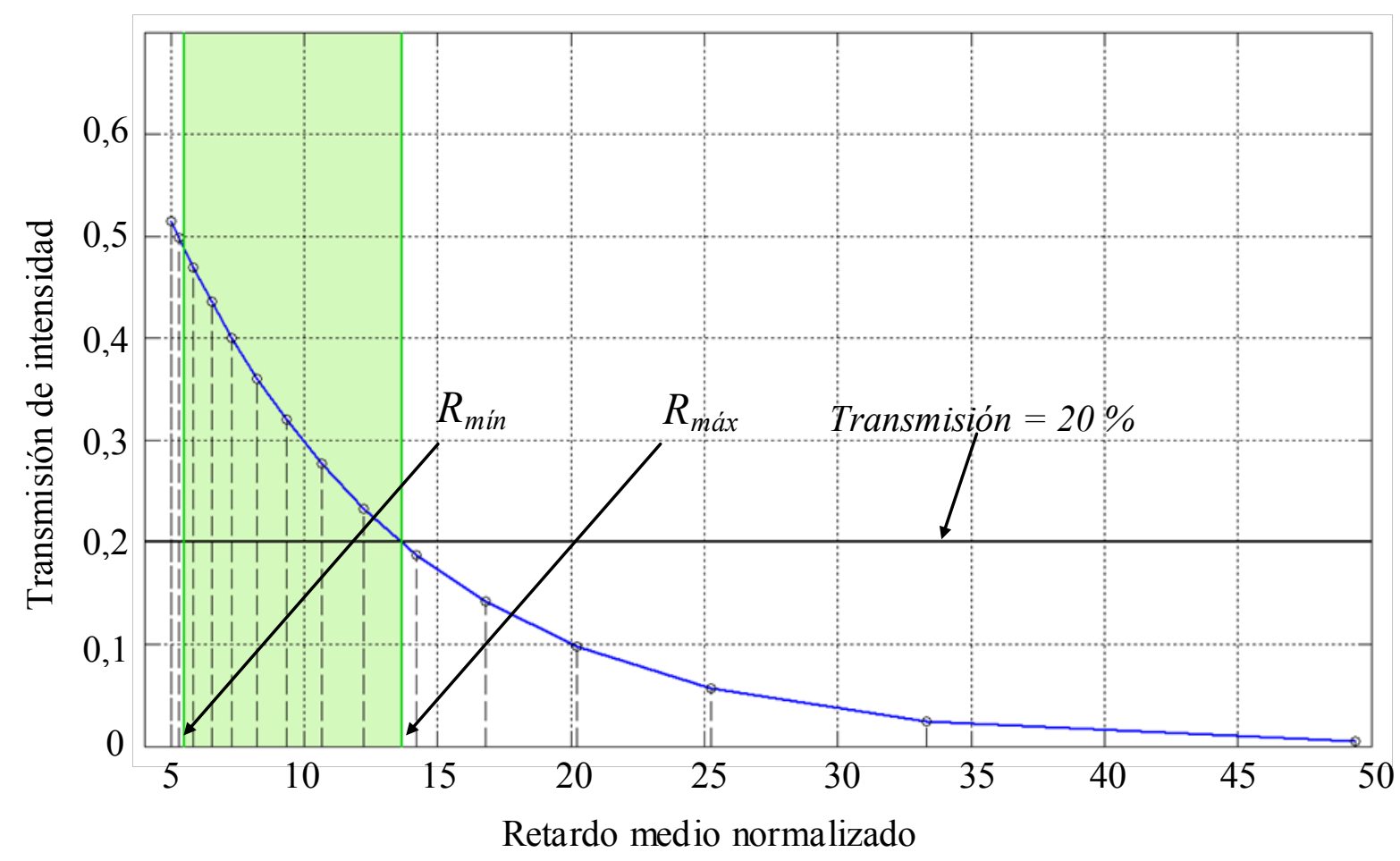

Figura 9.13: Transmisión de intensidad media en función del retardo medio normalizado.

\section{Factores de acoplamiento}

En la Fig. 9.14 se muestra la variación de los tres valores de acoplamiento involucrados en el control del sistema en función del retardo medio. Estas curvas permiten, dado un retardo medio, conocer los valores de los factores de acoplamiento necesarios para implementarlo, siempre teniendo en cuenta el criterio adoptado para la separación en frecuencia entre dos anillos adyacentes. En el mismo gráfico se indica también el rango de trabajo establecido anteriormente, que limita los factores de acoplamiento a valores entre 0,464 y 0,907 . 


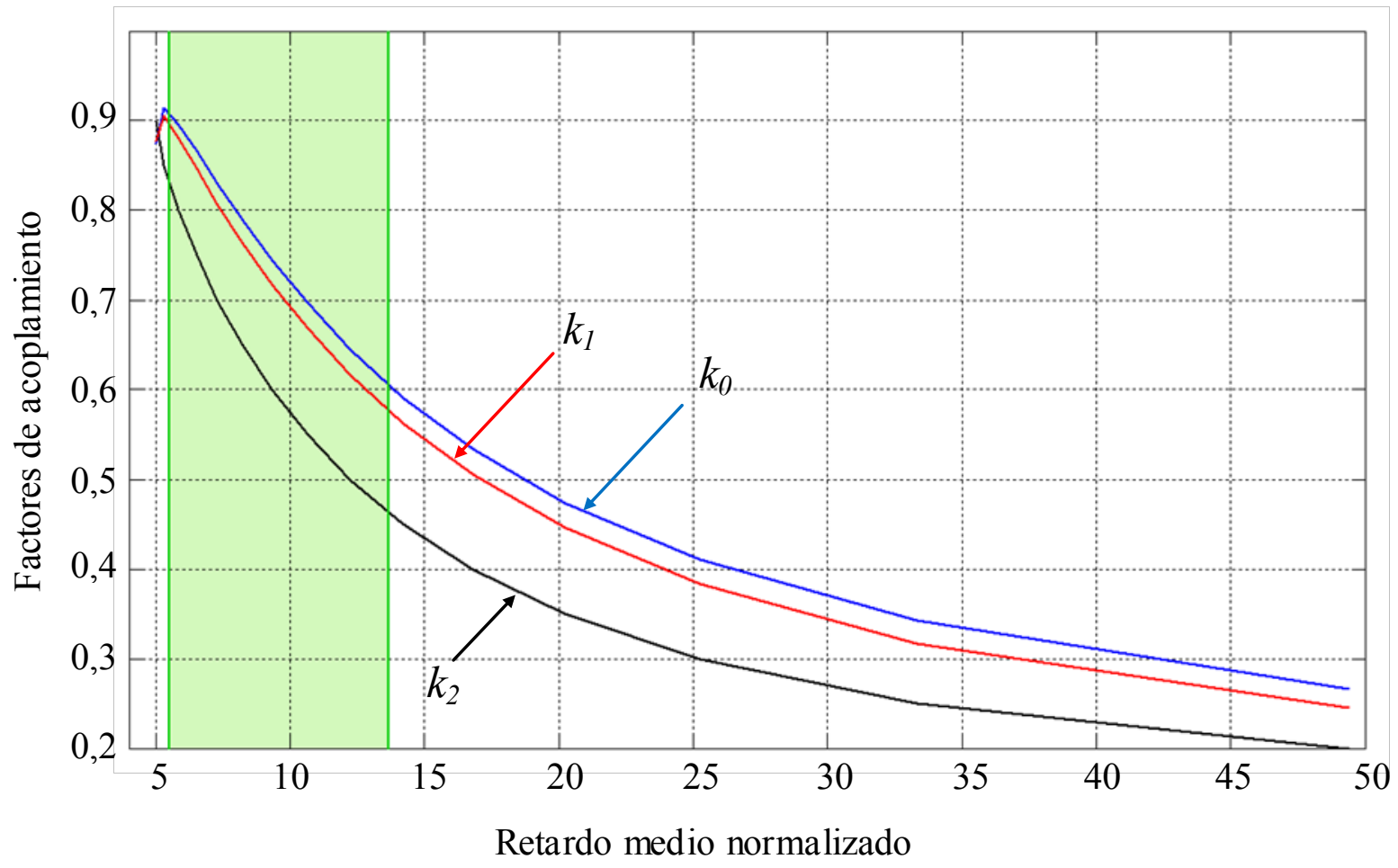

Figura 9.14: Factores de acoplamiento en función del retardo medio normalizado.

\section{Ripple}

En el gráfico de la Fig. 9.15 se muestra la variación del ripple en función del retardo medio implementado. Al igual que en el caso estudiado en el Capítulo 7, se observa que el crecimiento de $\Delta \tau$ es lento para valores relativamente bajos de $\bar{\tau}$, y se hace más rápido para retardos mayores, a partir de $14 \mathrm{~T}_{\mathrm{RT}}$ aproximadamente. 


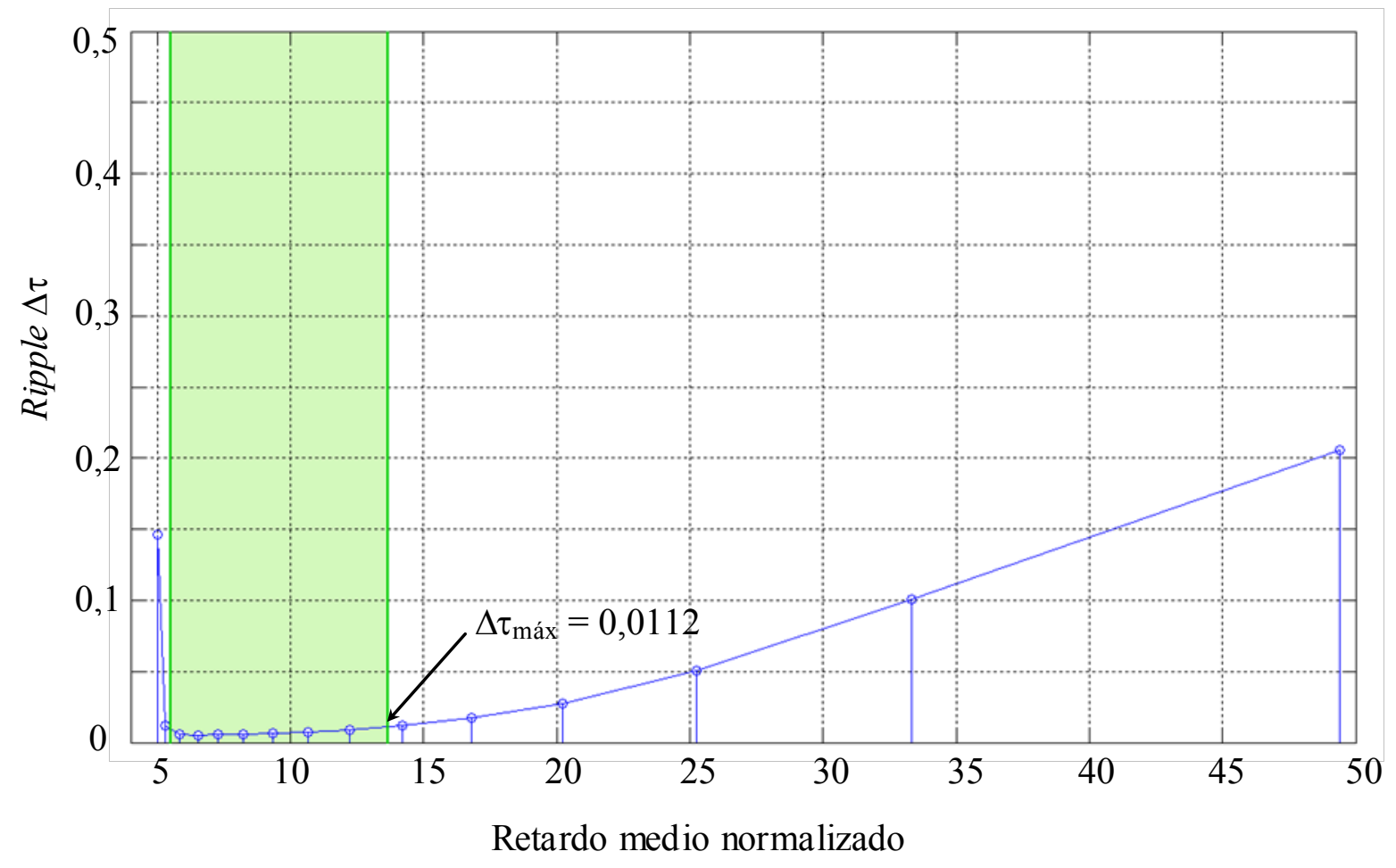

Figura 9.15: Variación del ripple en función del retardo medio normalizado.

Es para destacar que en todo el rango de retardos considerado, el ripple normalizado se mantuvo por debajo del valor 0,25 , es decir menor a la mitad del valor obtenido en el Capítulo 7 para un retardo medio de $8 \mathrm{~T}_{\mathrm{RT}}$. Y si, en cambio, se considera únicamente el rango de trabajo definido por cuestiones de potencia de la señal de salida, el ripple es más de 20 veces menor a dicha desviación. En particular, para el sistema considerado, el ripple máximo en el rango de trabajo es de $0,0112 \mathrm{~T}_{\mathrm{RT}}$, que corresponde a $2,08 \mathrm{ps}$ y se da para un retardo medio de 2,55 ns $\left(13,7 \mathrm{~T}_{\mathrm{RT}}\right.$ ). Esto demuestra la mejora (con respecto al ripple y retardo medio máximo que pueden implementarse) obtenida al sintonizar los anillos en función del retardo medio. 


\section{Ancho de banda de ripple}

En la siguiente figura se muestra la variación del ancho de banda del ripple con el retardo medio.

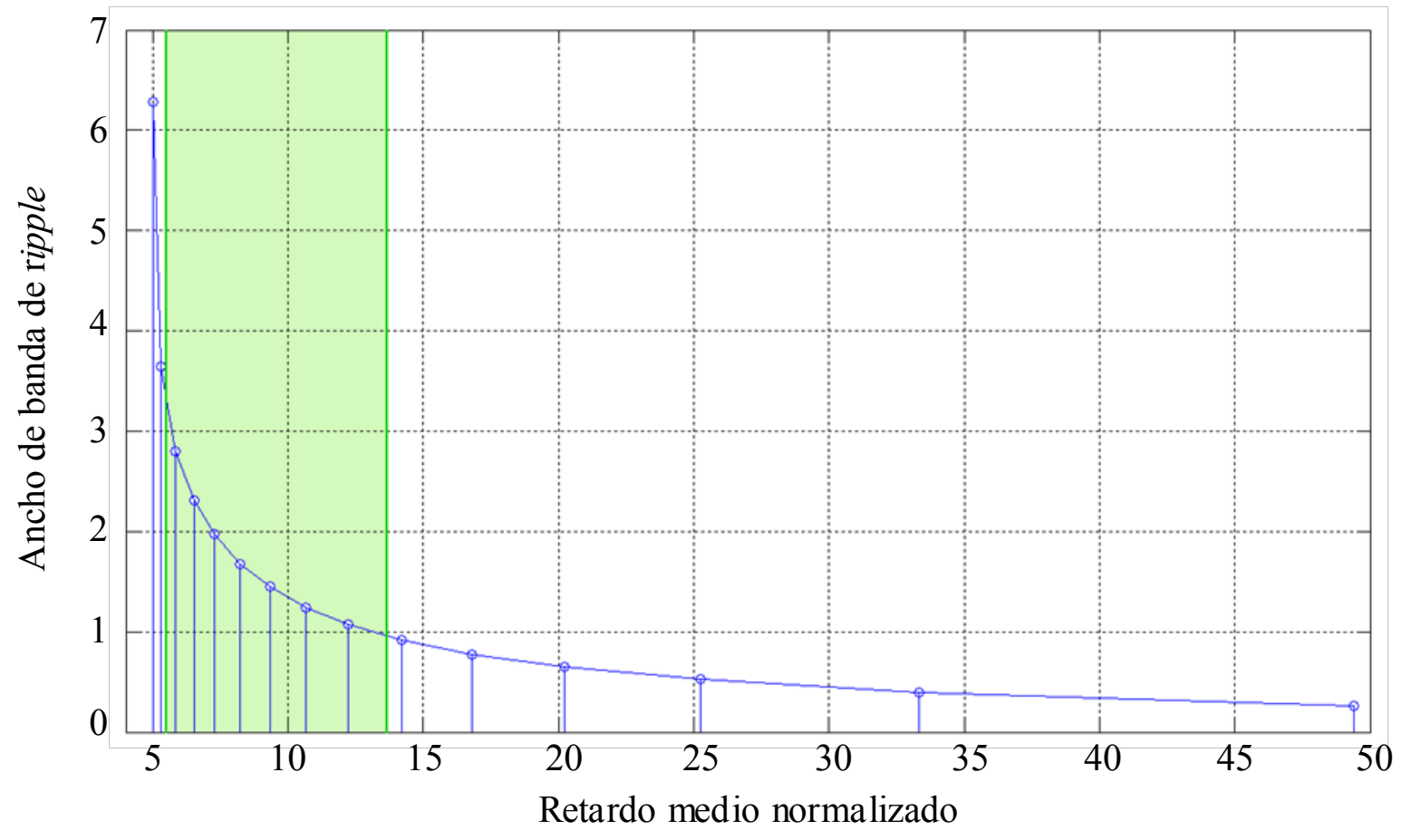

Figura 9.16: Variación del ancho de banda de ripple en función del retardo medio normalizado

Como se esperaba luego de los análisis de los capítulos previos, el ancho de banda disminuye a medida que se implementa un retardo medio mayor, y lo hace más rápidamente para retardos relativamente pequeños, que son aquellos que están dentro del rango de trabajo.

Teniendo en cuenta que el Free Spectral Range corresponde a un valor de $2 \pi$ en unidades de frecuencia angular normalizada, la variación del ancho de banda en el rango de trabajo abarca desde 53,06 \% hasta el 15,28 \% del FSR. Estos valores, aplicados al sistema considerado, corresponden a un ancho de banda mínimo de 0,82 $\mathrm{GHz}$ y máximo de 2,85 GHz. 


\subsection{Conclusiones}

En este capítulo se presentó un método que permite estimar los valores de los parámetros que controlan una línea de retardo implementada mediante la conexión en cascada de resonadores ópticos en anillo. El método consiste en generar tablas de look up a partir de datos obtenidos mediante simulaciones numéricas del sistema en las condiciones deseadas.

A modo de ejemplo, y para explicar el procedimiento, se aplicó esta estrategia a un sistema de cinco ORRs en cascada con las mismas características constructivas que las consideradas a lo largo de los capítulos anteriores. Los resultados obtenidos muestran que este método permite implementar retardos medios (desnormalizados) entre 1,02 ns con ancho de banda de 2,85 GHz, y 2,55 ns con ancho de banda de 0,82 GHz, siempre con variaciones menores a 2,08 ps.

Las curvas generadas que relacionan los parámetros de control del sistema con el retardo medio pueden ajustarse mediante cualquier método numérico, para luego generar las tablas de look up que se utilizarán en tiempo real. En particular, las curvas que se requiere ajustar son aquellas que relacionan los factores de acoplamiento con el retardo medio, y el FWHM de un ORR con el factor de acoplamiento. Esta última permite determinar los desplazamientos de fase de cada anillo.

Aunque dichas curvas se obtuvieron para el caso particular de un sistema determinado, la metodología puede aplicarse, en teoría, a cualquier número de resonadores de los que se conozcan sus características constructivas, requiriendo la simulación y el registro de las características del sistema en cada caso. Sin embargo, cabe mencionar que en la práctica, al aumentar el número de anillos también lo hace la complejidad. Para líneas de retardo compuestas por más de 5 anillos, el proceso iterativo para conseguir el ripple uniforme dentro de la banda de interés se vuelve más engorroso debido a la cantidad de factores de acoplamiento que hay que modificar. Por otro lado, el FSR de los anillos también limita la cantidad de resonadores que pueden conectarse en cascada, ya que si el ancho de banda que se requiere es demasiado grande, las respuestas de dos períodos consecutivos pueden llegar a superponerse. Para evitar esta superposición, y dependiendo del ancho de banda requerido, se deberá optar por anillos de dimensiones menores, es decir, con mayor FSR. 


\section{Capítulo 10 - Sistema de beamforming óptico basado en ORRs}

\subsection{Introducción}

En este capítulo se analizará un sistema óptico conformador de haz (Optical Beamformer (OBF)) basado en los dispositivos resonadores ORRs estudiados a lo largo de esta tesis. Como se explicó en el Capítulo 1, un OBF es un sistema que hace uso del procesamiento fotónico de señales para generar retardos temporales controlados en las señales que alimentan un arreglo de antenas.

Debido a que el sistema OBF puede asemejarse a un enlace óptico analógico entre la fuente de la señal que se desea transmitir y el arreglo de antenas que, en definitiva, la irradia, primero se presentará un enlace genérico representativo y se obtendrán las figuras de mérito que permitan analizar su desempeño. Para ello, antes se describirán los componentes principales que están presentes en este tipo de enlaces.

Luego, con el fin de analizar la capacidad del sistema propuesto para direccionar un haz de microondas, se presentarán simulaciones numéricas del patrón de radiación que permitan caracterizar los apartamientos del ángulo al trabajar con señales de banda ancha. 


\subsection{Análisis del desempeño de un enlace óptico analógico}

En el Capítulo 1 se realizó una introducción a los sistemas conformadores de haz, y en particular a aquellos que generan los retardos necesarios por medios ópticos, conocidos como Optical Beamformers. En esta sección se analiza el desempeño del enlace analógico que se establece entre la señal de radiofrecuencia (RF) de entrada y un elemento radiante del conjunto de antenas en un sistema OBF. Dicho enlace involucra los siguientes subsistemas: la generación de la señal eléctrica de RF, la conversión eléctrica a óptica, la propagación y el procesamiento fotónico para generar los retardos reales que controlan el patrón de radiación, la conversión óptica a eléctrica, y finalmente, el acondicionamiento de la señal de RF para la posterior excitación del elemento del arreglo de antenas. Para cada elemento se puede analizar un enlace y para ello primero se introducirán los conceptos y subsistemas involucrados.

Para simplificar el problema, se considerará solamente el enlace en modo de transmisión, es decir, que la señal es irradiada por el PAA. Un análisis similar se puede plantear para el sistema en modo de recepción, donde la señal de entrada es recibida en la antena, para lo cual se debe modificar levemente la arquitectura del OBF.

\section{Esquemas de transporte}

Los enlaces ópticos analógicos, también conocidos como enlaces de radio sobre fibra (RoF, del inglés Radio over Fiber), se encargan de transportar las ondas de radio analó gicas a través de la fibra óptica (FO). Existen diferentes implementaciones del enlace de RoF, a saber:

1) Sistemas de RF sobre FO (RFoF): La señal de RF eléctrica es usada para modular la portadora óptica. La señal modulada es transportada por la FO y luego detectada en el fotodiodo del receptor, acondicionada (filtrada y amplificada) y transmitida de manera inalámbrica.

2) Sistemas es de frecuencia intermedia (IF) sobre FO (IFoF): La señal de RF eléctrica es convertida a una frecuencia intermedia menor, la cual es usada para modular la portadora óptica. Luego de ser transportada por la FO hasta el fotodetector del 
receptor, la señal de IF es convertida nuevamente a RF, acondicionada y transmitida de manera inalámbrica.

3) Sistemas de banda base $(\mathrm{BB})$ sobre FO $(\mathrm{BBoF})$ : En este caso la portadora óptica se modula con la señal analógica en banda base, o su versión digitalizada. Dicha señal es transportada por la fibra y luego recibida en el fotodetector. La señal en banda base es acondicionada, trasladada a RF y luego transmitida inalámbricamente. Si la transmisión es digital, previamente es necesario convertirla nuevamente a analógica.

La Fig. 10.1 muestra los tres esquemas de transporte óptico de la señal analógica, y los espectros correspond ientes a cada una de las señales que se transmiten por la FO.

La opción de RFoF es la más sencilla porque no requiere el agregado de componentes adicionales. No obstante, posee la desventaja de que las señales se encuentran en alta frecuencia y requiere de transmisores y receptores ópticos de mayor ancho de banda y rango dinámico que en las soluciones de IFoF o BBoF. Por otro lado, RFoF también es más sensible a sufrir dispersión cromática por la propagación en la fibra óptica debido al ancho de banda mayor que ocupa la señal modulada. En este trabajo se considera el caso de un sistema de RFoF para transmitir las señales de OBF.

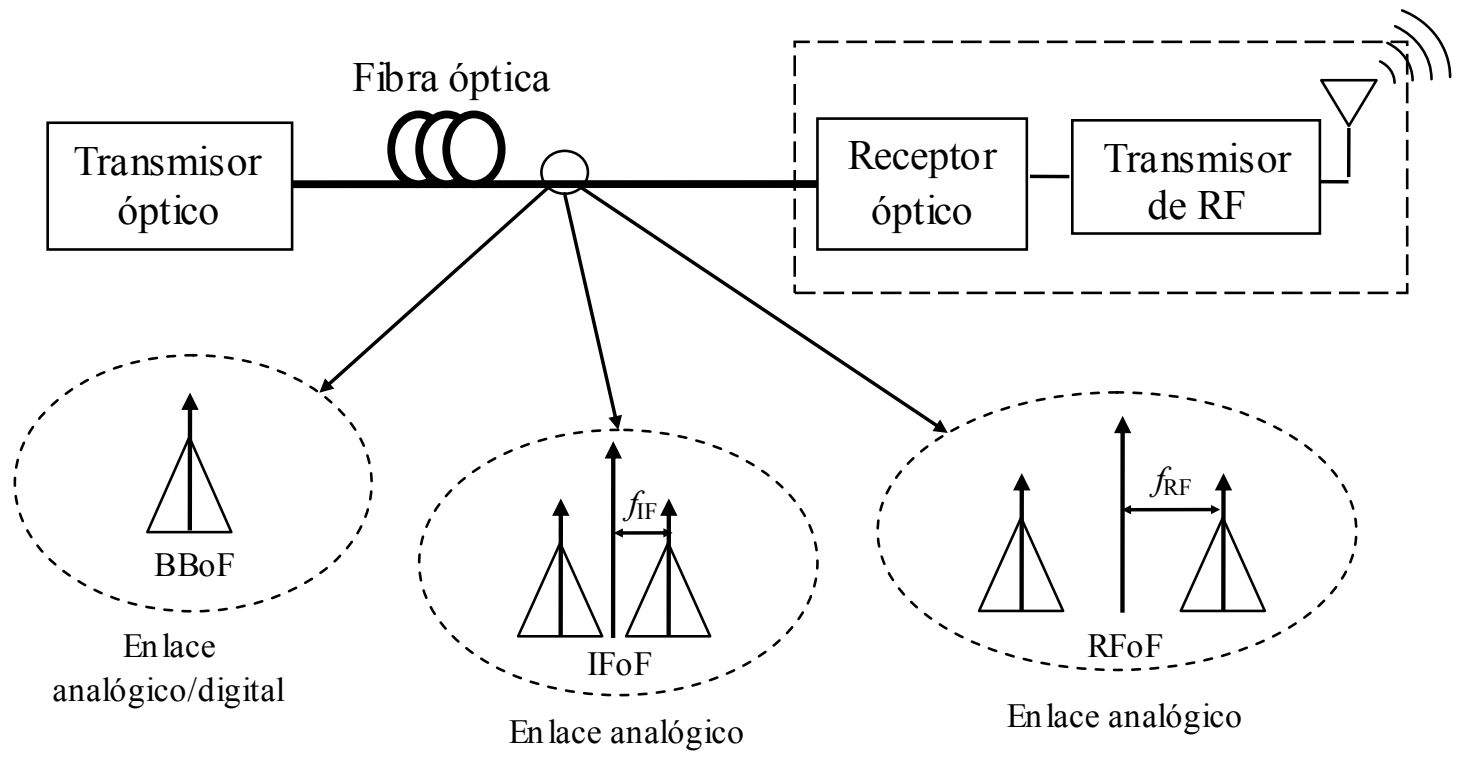

Figura 10.1: Esquemas de transporte óptico de una señal analógica. 


\section{Transmisor y receptor ópticos}

La transmisión óptica puede implementarse mediante la modulación directa de la fuente óptica, que puede ser un diodo láser (Laser Diode (LD)), o mediante la modulación externa de la portadora óptica. Cualquiera de estos dos métodos puede ser usado para realizar la modulación de la intensidad (Intensity Modulation (IM)) (o la potencia óptica). Por otro lado, el proceso asociado a la conversión óptica eléctrica se denomina detección directa (Direct Detection (DD)) y se realiza mediante un fotodetector. Debido a su simplicidad, los sistemas de modulación de intensidad y detección directa (IM/DD) son los más comúnmente utilizados.

La Fig. 10.2(a) muestra el esquema de modulación directa, donde la señal óptica es obtenida utilizando una señal eléctrica para modular directamente la corriente del láser. De esta manera, esta corriente queda compuesta por una corriente continua de polarización y una corriente moduladora de RF, como puede verse en la Fig. 10.2(b) mediante la curva característica P-I del láser.

En contraposición, la Fig. 10.3(a) muestra el esquema de modulación externa, donde el láser es polarizado con una corriente constante y emite una onda continua (Continuous Wave $(\mathrm{CW})$ ), y posteriormente un modulador electroóptico alimentado con la señal de RF es usado para modular la señal óptica. La Fig. 10.3(b) muestra el esquema de un modulador electroóptico típico de niobato de litio $\left(\mathrm{LiNbO}_{3}\right)$. Este modulador consiste en un interferómetro Mach-Zehnder (MZI) en el cual la señal óptica de entrada es dividida en partes iguales para cada rama y transmitida por las guías de onda. Luego de la propagación (de unos pocos centímetros), las dos guías se recombinan, haciendo que las señales interfieran. Si se genera un cambio de fase relativo entre las señales que se propagan por las dos ramas, es posible variar el patrón de interferencia o, equivalentemente, generar una modulación de amplitud. Esto se logra mediante la aplicación de un campo eléctrico (modulación de RF) en las guías, lo que produce un cambio de índice de refracción y por consiguiente un cambio de fase de la señal óptica. La Fig. 10.3(c) muestra la transmisión óptica en función de la tensión aplicada, Vi. Puede observarse que cuando se aplica una tensión de 0 volts la transferencia es máxima debido a que la interferencia entre las señales de las dos ramas es constructiva, mientras que al aplicar una tensión determinada, denominada $\mathrm{V}_{\pi}$, la transmisión es nula, ya que el desfasaje de los campos es de $180^{\circ}$ y la 
interferencia resulta destructiva. De esta manera, para trabajar en la zona lineal del MZI, la tensión continua que lo alimenta más la señal de RF no debe superar el valor de $\mathrm{V}_{\pi}$.

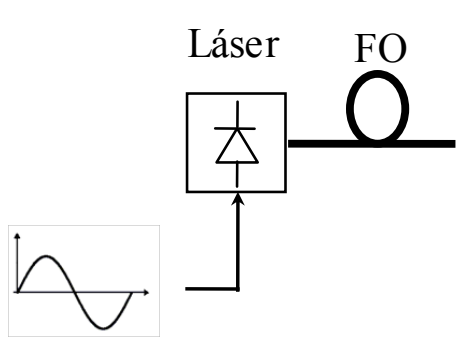

(a)

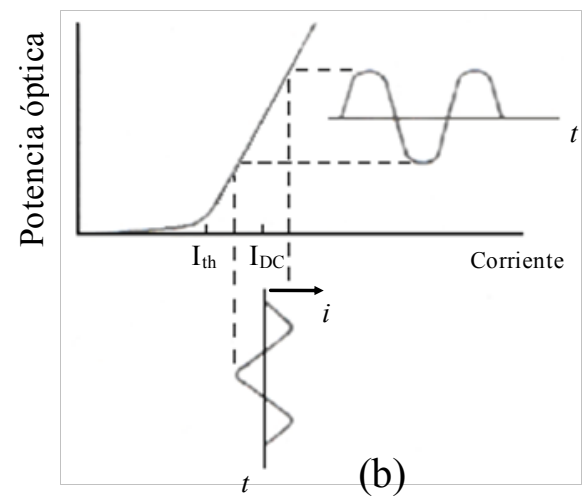

(b)

Figura 10.2: (a) Esquema de modulación directa; (b) Curva P-I del diodo láser.

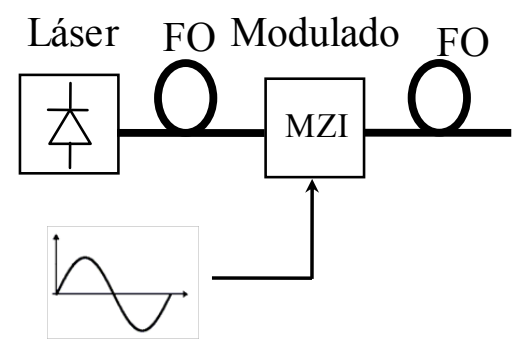

(a)

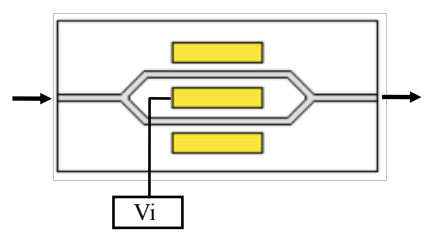

(b)

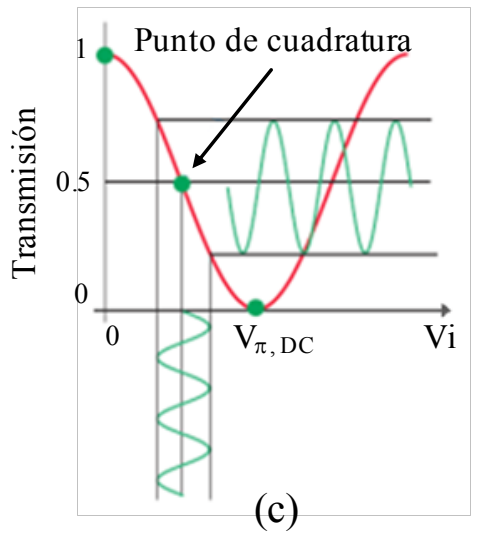

(c)

Figura 10.3: (a) Esquema de modulación externa; (b) Modulación elecroóptica mediante un MZI; (c) Transmisión óptica del modulador en función de la tensión aplicada.

Como se mencionó, la fuente óptica puede consistir en un diodo láser, pero también en un LED (Light Emitting Diode). Sin embargo, este último posee tiempo de respuesta y ancho espectral muy grandes, lo que dificulta la operación en sistemas de gran ancho de banda. Por otro lado, los diodos láser pueden trabajar a mayor velocidad. Particularmente existen láseres de semiconductor con realimentación distribuida (Distributed Feedback Laser (DFB Laser)), los cuales poseen ancho espectral muy chico, menores al MHz, y tiempos de respuesta muy bajos, lo que los hace ideales para sistemas de gran ancho de 
banda (hasta decenas de GHz). Algunos de los parámetros importantes a tener en cuenta en el diseño del sistema son: el ancho espectral, el tiempo de respuesta, el ruido de intensidad relativa, la eficiencia de conversión eléctrica a óptica y la linealidad. La Fig. 10.4(a) muestra un láser DFB típico con salida acoplada a una fibra óptica y encapsulado mariposa de 14 pines.

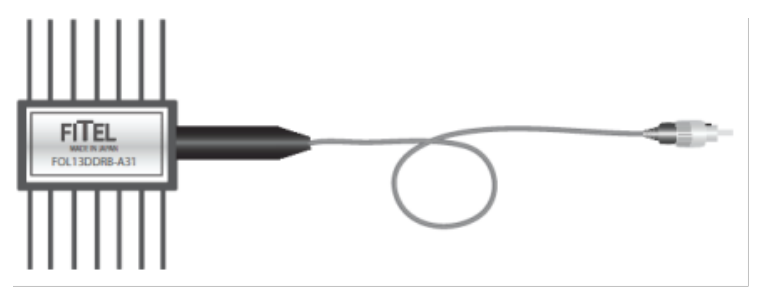

(a)

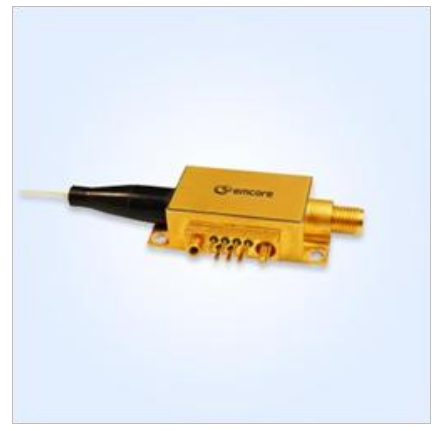

(b)

Figura 10.4: (a) Diodo láser DBF típico; (b) Fotodiodo PIN con entrada de fibra y salida SMA.

El receptor óptico puede implementarse con un fotodiodo PIN o un fotodiodo de avalancha (Avalanche Photodiode (APD)). En este trabajo se considerarán del tipo PIN dado que son dispositivos más económicos y de mayor ancho de banda que los APD, aunque estos últimos poseen mayor ganancia y mejor sensibilidad. Algunos de los parámetros importantes que deben ser tenidos en cuenta son: el ancho de banda, la responsividad (capacidad de convertir la potencia óptica recibida en una corriente eléctrica), la sensibilidad y el ruido que introduce. La Fig. 10.4(b) muestra un fotodiodo PIN con entrada óptica acoplada por fibra y salida eléctrica en el conector SMA (SubMiniature version A), que es un tipo de conector roscado para cable coaxial utilizado en microondas.

Un modelo sencillo de pequeña señal para representar la señal óptica modulada en amplitud está dado por la siguiente ecuación:

$$
e(t)=E \exp \left(j \omega_{0} t\right)[1+m(t)]
$$


donde $E$ es la amplitud de la portadora óptica de frecuencia angular $\omega_{0}, m(t)$ es la señal de modulación, que en el caso de modulación de amplitud debe ser real y cumplir $|m(t)|<<1$.

La intensidad óptica, medible a partir de la corriente en el fotodiodo del receptor, es proporcional a $|e(t)|^{2}$, y aceptando que en régimen de pequeña señal $|m(t)|^{2}$ es despreciable frente a $m(t)$, puede escribirse:

$$
p(t) \propto E^{2}[1+2 m(t)]
$$

\section{Canal de fibra óptica}

El canalde fibra óptica es el medio por el cual se propaga la portadora óptica modulada por la señal de RF. Este canal se caracteriza por tener un gran ancho de banda y una muy baja atenuación.

La FO puede ser categorizada dependiendo de los modos de propagación que admiten en fibras monomodo (Single Mode Fiber (SMF)) y multimodo (Multi Mode Fiber (MMF)). Una de las principales diferencias entre ambas fibras radica en el diámetro del núcleo, el cual es de alrededor de $10 \mu \mathrm{m}$ para SMF y de 50-65 $\mu \mathrm{m}$ para MMF. Como su nombre lo indica, las SMF admiten la propagación únicamente del modo fundamental para ciertas longitudes de onda, mientras que las MMF admiten múltiples modos para cada longitud de onda. Esta particularidad hace que en las MMF exista una dispersión modal durante la propagación, debido a que cada modo se propaga a una velocidad diferente. Por otro lado, en las SMF un único modo se propaga y no existe la dispersión modal. Esto hace que la capacidad de transmisión de los sistemas IM/DD sea mucho mayor en SMF que en MMF. En este trabajo se considerará un canal de SMF dado que se espera transmitir señales cuyos anchos de banda superan los GHz.

Para analizar un enlace de RFoF que emplea SMF, dos aspectos del canal deben tenerse en cuenta inicialmente: la atenuación y la dispersión cromática de la fibra. La Fig. 10.5(a) muestra el perfil del coeficiente de atenuación, $\alpha$, de la SMF en función de la longitud de onda, $\lambda$, expresado en $\mathrm{dB} / \mathrm{km}$. Puede observarse que la mínima atenuación se obtiene alrededor de $1550 \mathrm{~nm}$ (banda C) y es aproximadamente $0,2 \mathrm{~dB} / \mathrm{km}$. Por otro lado, la Fig. 10.5(b) muestra el parámetro de dispersión cromática, $D$, de la SMF en función de la 
longitud de onda, expresado en $\mathrm{ps} /(\mathrm{km} \cdot \mathrm{nm})$. Dicho coeficiente está formado por la suma de la dispersión de guiado y la dispersión del material. Puede observarse que la SMF posee el cero de dispersión aproximadamente a $1300 \mathrm{~nm}$, donde se ubica la segunda ventana de transmisión de acuerdo al perfil de atenuación. Por otro lado, a $1550 \mathrm{~nm}$ el coeficiente de dispersión toma un valor de $16 \mathrm{ps} /(\mathrm{km} \cdot \mathrm{nm})$. Esto significa que, en esa longitud de onda, un pulso transmitido se ensanchará 16 ps por cada kilómetro de propagación en SMF y por cada nanómetro de ancho de banda de la fuente óptica.

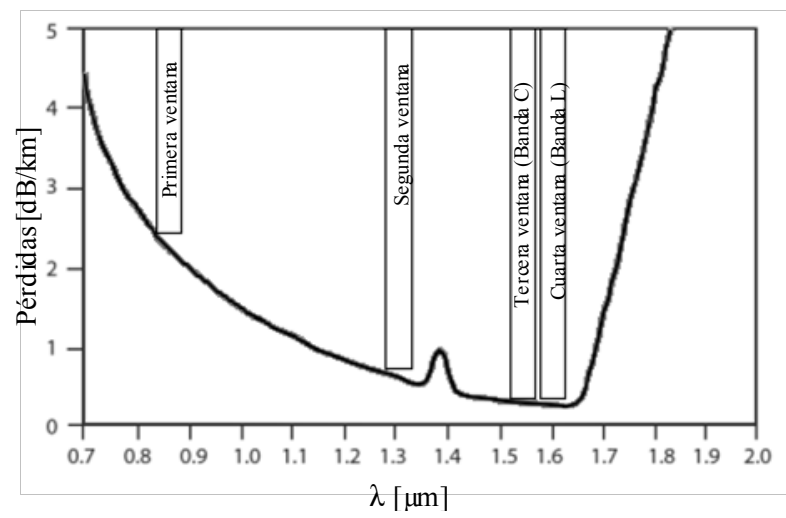

(a)

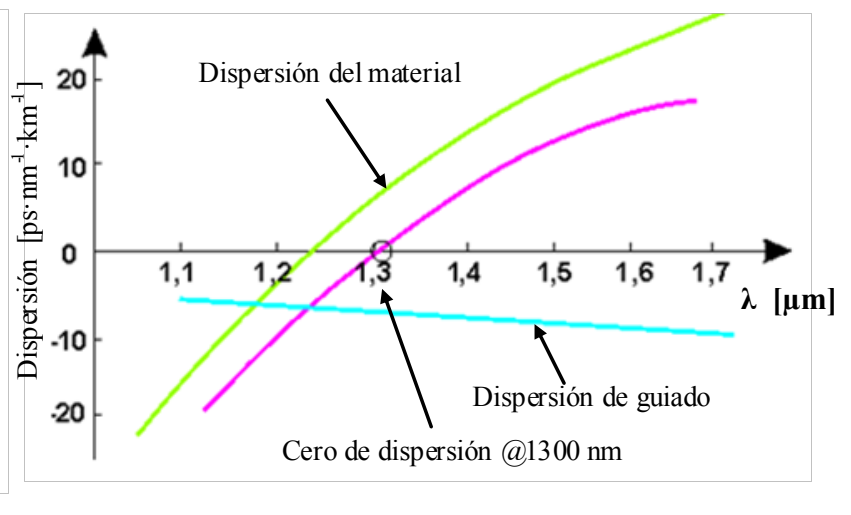

(b)

Figura 10.5: Curvas características de una SMF. (a) Atenuación; (b) Dispersión cromática.

De esta manera, un modelo de propagación en la FO lineal alrededor de la portadora óptica láser se puede esquematizar mediante la siguiente función de transferencia:

$$
H_{F O}(\omega)=\exp (-\alpha L / 2) \exp \left[-j\left(\omega \beta_{1}+\omega^{2} \beta_{2} / 2\right) L\right]
$$

En la expresión anterior, $L$ es la distancia de propagación en la $\mathrm{FO}, \beta_{1}$ y $\beta_{2}$ son los coeficientes de dispersión de primer y segundo orden respectivamente. El coeficiente $\beta_{1}=1 / v_{\mathrm{g}}$ es la inversa de la velocidad de grupo, $v_{\mathrm{g}}$, mientras que $\beta_{2}=\partial\left(1 / \nu_{\mathrm{g}}\right) / \partial \omega$ es la variación de la inversa de la velocidad de grupo. La Ec. (10.3) muestra que el primer factor, dado por la exponencial real, produce una atenuación de la señal de entrada, mientras que el segundo factor, dado por la exponencial con argumento complejo, produce el desfasamiento de la misma. Particularmente, el primer término de la fase es lineal con la frecuencia y no genera distorsión, mientras que el segundo término, cuadrático con la frecuencia, produce una distorsión lineal de fase. 
El coeficiente de dispersión de segundo orden, $\beta_{2}$, está relacionado con el parámetro de dispersión $D$, que se muestra en la Fig. 10.5(b), a través de la siguiente expresión:

$$
D=-\frac{2 \pi c}{\lambda^{2}} \beta_{2}
$$

La red óptica de conformación de haces (OBFN, OBF Network), se encarga de producir retardos de grupo ajustables de manera de alimentar las antenas con las señales de RF que posean fases o retardos relativos apropiados. El resto del enlace óptico lo constituye la FO que alimenta la OBFN y la FO transporta la señal desde éste hasta el fotodetector.

Como se dijo previamente, la fibra óptica posee una atenuación extremadamente baja, de manera que puede despreciarse el factor correspondiente en la función de transferencia. No obstante, si las pérdidas son apreciables, las mismas son sencillamente compensadas con amplificadores ópticos de fibra dopada con erbio (Erbium Doped Fiber Amplifier (EDFA)), los cuales se agregan dentro del enlace. Por otro lado, si se considera que cada canal de FO asociado a un elemento del arreglo introduce un retardo de grupo constante e igual para todos los elementos dado por $T g_{\mathrm{i}}=\beta_{1} L_{\mathrm{i}}$, donde $\mathrm{i}=1 \ldots \mathrm{N}$ es el enlace de $\mathrm{FO}$ asociado al elemento i del arreglo, entonces el término de la función de transferencia de la FO correspondiente a $\beta_{1}$ puede no ser considerado en este análisis. Por lo tanto, una función de transferencia simplificada de la FO que permite analizar los efectos de la dispersión cromática para determinar el ancho de banda del canal puede escribirse como:

$$
H_{F O-C D}(\omega)=\exp \left(-j \omega^{2} L \beta_{2} / 2\right)
$$

\section{Análisis del desempeño}

Para analizar el desempeño del enlace de RFoF se considerarán por un lado los efectos dispersivos, los cuales limitarán el ancho de banda de la señal a transmitir, y por el otro, el ruido y los efectos no lineales, que limitarán la potencia transmitida.

La Fig. 10.6 muestra el esquema general del enlace de RFoF analizado y los efectos que se tendrán en cuenta para analizar el desempeño. 


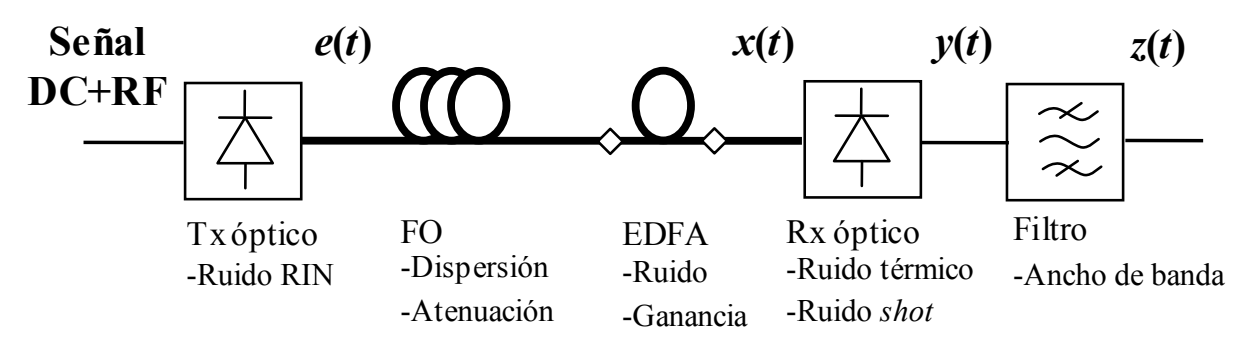

Figura 10.6: Esquema general del enlace de RFoF analizado.

\section{1) Limitación del ancho de banda debido a la dispersión cromática}

En referencia a la Fig. 10.6, si se considera la señal óptica modulada en amplitud $e(t)$ de la Ec. (10.1), con la modulación de RF dada por

$$
m(t)=A \cos \left(\omega_{\mathrm{RF}} t\right),
$$

y si se considera que el EDFA no introduce dispersión y su ganancia es constante en la banda de interés, luego de propagarse por la FO, cuya función de transferencia está dada por la Ec. (10.5), al fotodetector llega la señal $x(\mathrm{t})$. En el dominio de la frecuencia, esta señal está representada por $X(\omega)$, que es su Transformada de Fourier (TF):

$$
\begin{aligned}
& X(\omega)=\left[2 \pi E \cdot \delta\left(\omega-\omega_{0}\right)+2 \pi^{2} E A \cdot \delta\left(\omega-\omega_{0}-\omega_{R F}\right)+2 \pi^{2} E A\right. \\
& \delta \omega-\omega O+\omega R F \cdot \exp -j \omega 2 L \beta 22
\end{aligned}
$$

La salida del fotodetector es proporcional a $|x(\mathrm{t})|^{2}$, por lo que, si se considera unitaria la constante de proporcionalidad para simplificar la notación, se puede escribir:

$$
y(t)=|x(t)|^{2}=x(t) x^{*}(t)
$$

En el dominio de la frecuencia, la expresión anterior se puede calcular como la convolución de las respectivas $\mathrm{TF}$ de la siguiente manera:

$$
\begin{gathered}
Y(\omega)=\frac{1}{2 \pi}\left\{X(\omega) * X^{*}(-\omega)\right\} \\
Y(\omega)=\frac{1}{2 \pi}\left\{\left[2 \pi E \cdot \delta\left(\omega-\omega_{0}\right)+2 \pi^{2} E A \cdot \delta\left(\omega-\omega_{0}-\omega_{R F}\right)+2 \pi^{2} E A \cdot \delta\left(\omega-\omega_{0}+\right.\right.\right. \\
\left.\left.\left.\omega_{R F}\right)\right] \cdot \exp \left(-j \omega^{2} L \frac{\beta_{2}}{2}\right)\right\} \times\left\{\left[2 \pi E \cdot \delta\left(-\omega+\omega_{0}\right)+2 \pi^{2} E A \cdot \delta\left(-\omega+\omega_{0}+\omega_{R F}\right)+\right.\right. \\
\left.\left.2 \pi^{2} E A \cdot \delta\left(-\omega+\omega_{0}-\omega_{R F}\right)\right] \cdot \exp \left(j \omega^{2} L \frac{\beta_{2}}{2}\right)\right\}=Y_{\omega_{R F}}(\omega)+Y_{D C}(\omega)+Y_{2 \omega_{R F}}(\omega)
\end{gathered}
$$


Luego de trabajar matemáticamente en la ecuación anterior, se pueden diferenciar tres grupos de términos: uno centrado en DC (corriente continua, del inglés Direct Current), uno en $\pm \omega_{\mathrm{RF}}$, y otro en $\pm 2 \omega_{\mathrm{RF}}$. Posteriormente, un filtro se encarga de eliminar las componentes de DC y del doble de frecuencia, y se puede demostrar que la potencia de radiofrecuencia la salida, $P_{R F}$, cumple que

$$
P_{R F} \propto \cos ^{2}\left[\pi c D L\left(f_{R F} / f_{0}\right)^{2}\right]
$$

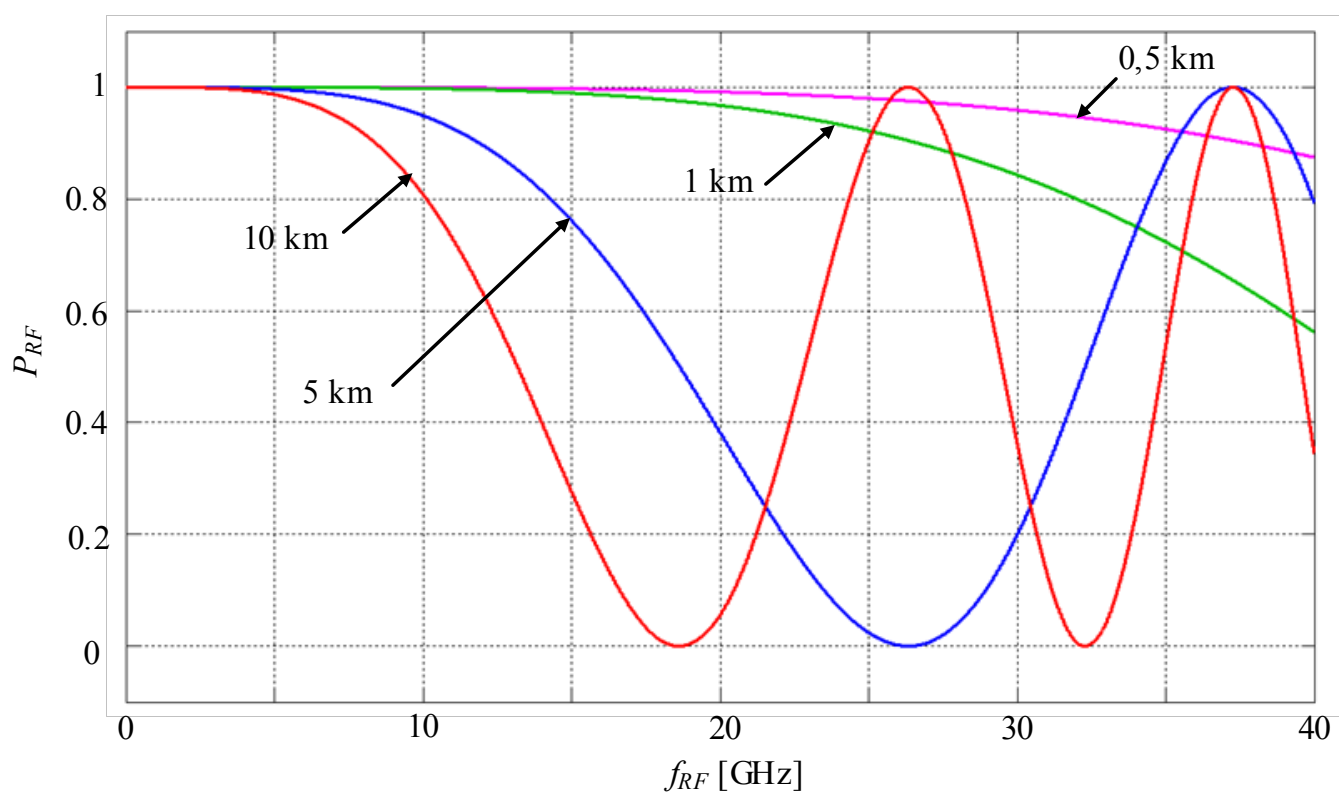

Figura 10.7: Potencia de RF a la salida del receptor en función de $f_{\mathrm{RF}}$ y con la longitud de la fibra óptica, $L$, como parámetro.

En la Fig. 10.7 se observa el gráfico de $P_{R F}$ en función de $f_{R F}$ para distintas longitudes de fibra. En particular, el parámetro $L$ toma los valores: 0,5; 1; 5 y $10 \mathrm{~km}$. Como se aprecia, la dispersión cromática de la fibra produce una atenuación de la señal de RF fotodetectada que limita el ancho de banda de la señal a transmitir.

Con el fin de evaluar la degradación de la señal, se puede definir la penalidad de potencia debida a la dispersión cromática, $P e n_{d c}$, en decibeles como

$$
P e n_{d c}=10 \log \left|\frac{P_{R_{\text {fibra }}}}{P_{R_{\text {nofibra }}}}\right|
$$



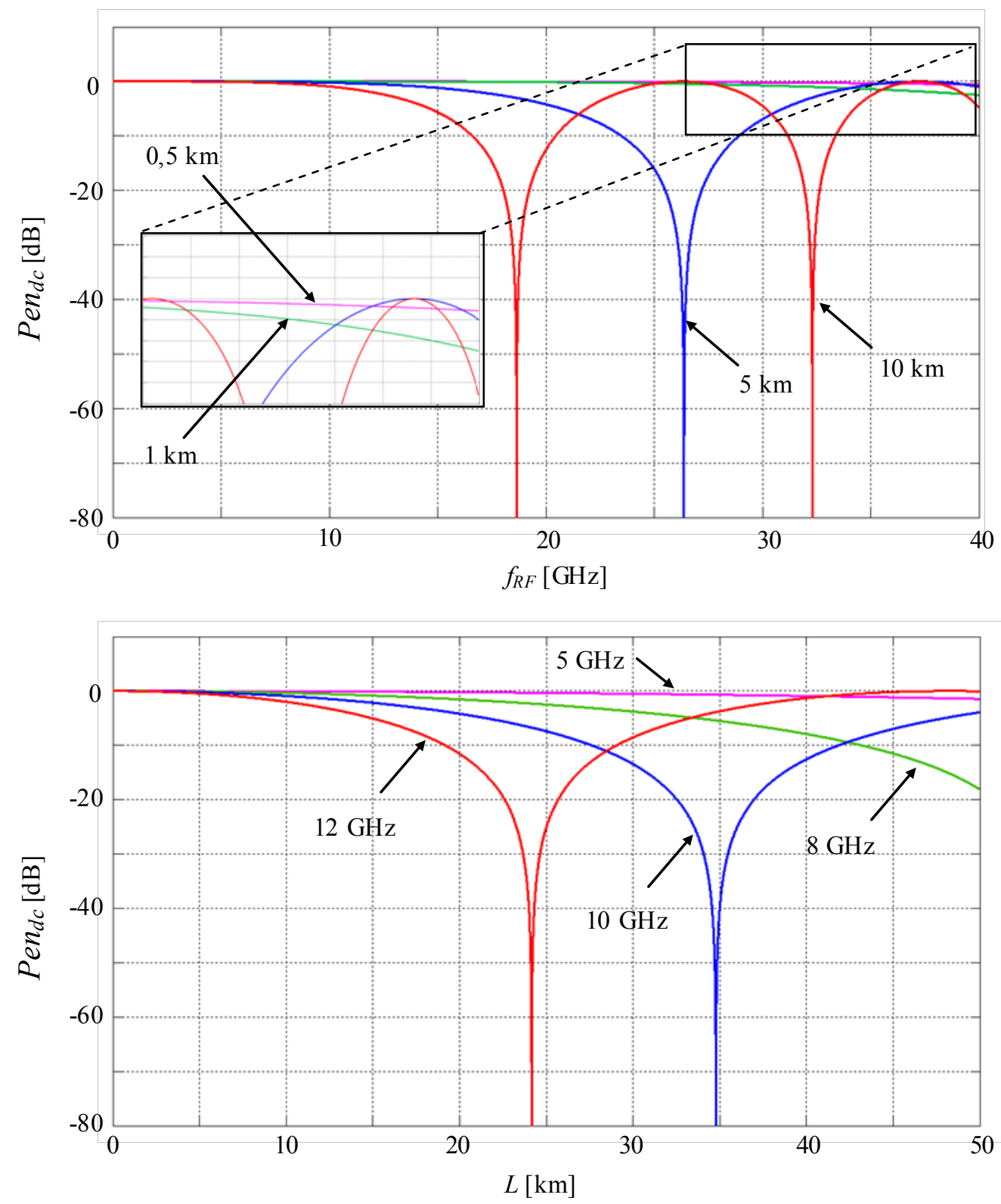

Figura 10.8: Penalidad de potencia, $P e n_{d c}$. (a) En función de la frecuencia, con parámetro $L$; (b) En función de la longitud de la fibra, con parámetro $f_{\mathrm{RF}}$.

En la Fig. 10.8(a) se observa la penalidad de potencia en función de la frecuencia $f_{\mathrm{RF}}$ con la longitud de la fibra como parámetro. Para las frecuencias que se consideraron en este trabajo, de alrededor de $10 \mathrm{GHz}$, la penalidad o pérdidas debidas a la dispersión cromática alcanzan los valores aproximados de 0,$00 ; 0,01 ; 0,22$ y $0,92 \mathrm{~dB}$ para fibras de longitud 0,5; $1 ; 5$ y $10 \mathrm{~km}$, respectivamente. 
Por otro lado, la Fig. 10.8(b) muestra la penalidad en función de la longitud de la fibra para distintos valores de frecuencia $f_{\mathrm{RF}}$. Teniendo en cuenta lo antes dicho sobre las frecuencias que se manejan en este trabajo, si se utilizan tramos de fibra de longitud menor a 7,2 km, las pérdidas debidas a la dispersión cromática de la fibra se mantienen menores a $1 \mathrm{~dB}$ para frecuencias de alrededor de $10 \mathrm{GHz}$.

\section{2) Limitación de la relación señal a ruido debido a las pérdidas}

Para calcular las limitaciones de la relación señal a ruido, se considera el sistema mostrado en la Fig. 10.6. En un primer análisis se discute el enlace de RFoF sin el amplificador óptico.

La potencia de entrada a la FO es

$$
p_{e}(t)=P_{e}\left[1+M_{0} s(t)\right]
$$

donde $P_{e}$ es la potencia óptica media a la entrada, $M_{0}$ es el índice de modulación óptica y $s(\mathrm{t})$ es la señal de modulación de RF normalizada y con media nula.

Las pérdidas ópticas (Optical Losses (OL)) del canal se obtienen al sumar las distintas contribuciones de las partes que lo componen, como se indica en la Ec. (10.14), donde $\alpha[\mathrm{dB} / \mathrm{km}]$ y $L[\mathrm{~km}]$ son el coeficiente de atenuación y la longitud de la $\mathrm{FO}$, respectivamente, $P_{\text {mod }}[\mathrm{dB}]$ las pérdidas de inserción del modulador externo, $N_{c o n}$ el número de conectores, $P_{c o n}[\mathrm{~dB}]$ las pérdidas por conector, $N_{e m p}$ el número de empalmes, $P_{e m p}[\mathrm{~dB}]$ las pérdidas por empalme y $I L_{O B F N}[\mathrm{~dB}]$ las perdidas por inserción de la red de conformación óptica de haces.

$$
O L[d B]=\alpha L+P_{\text {mod }}+N_{c o n} P_{c o n}+N_{e m p} P_{e m p}+I L_{O B F N}
$$

Por lo tanto, considerando al canal óptico lineal y sin distorsión, la potencia a la entrada del detector puede obtenerse a partir de la Ec. (10.15), donde $P_{\mathrm{x}}$ es la potencia óptica media recibida.

$$
p_{x}(t)=p_{e}(t) 10^{-O L[d B] / 10}=P_{x}\left[1+M_{0} s(t)\right]
$$

El fotodetector PIN, de responsividad $\mathfrak{R}$, convierte la potencia óptica en una corriente, $i_{\mathrm{Y}}(t)$, que se compone de un valor medio $I_{Y}$ y un valor AC dado por $i_{y}(\mathrm{t})($ Ec. $(10.16))$. 


$$
i_{Y}(t)=\Re p_{x}(t)=\Re P_{x}+\Re P_{x} M_{0} s(t)=I_{Y}+i_{y}(t)
$$

Luego, la corriente es acondicionada en el receptor mediante un filtro y, eventualmente, un amplificador de transimpedancia (Transimpedance Amplifier (TIA)). El filtro elimina la componente de DC y limita el ancho de banda de ruido del receptor. De esta manera, la potencia de señal eléctrica estará dada por la Ec.(10.17), y si la señal de entrada normalizada de RF es sinusoidal, resulta en la Ec. (10.18).

$$
\begin{gathered}
S=\left(\Re P_{x} M_{0}\right)^{2}\left\langle s^{2}(t)\right\rangle \\
C=\frac{1}{2}\left(\Re P_{x} M_{0}\right)^{2}
\end{gathered}
$$

Para calcular la relación señal a ruido es necesario cuantificar cada una de las contribuciones de ruido que estarán presentes en el sistema. El fotodetector recibirá fotones provenientes de la señal óptica de entrada los cuales generarán electrones y éstos la corriente. Esta corriente posee una componente aleatoria denominada ruido cuántico o shot, cuya varianza es

$$
\sigma_{s}^{2}=2 q I_{Y} B_{e}
$$

donde $q=1,602 \times 10^{-19} \mathrm{C}$ es la carga del electrón y $B_{e}$ es el ancho de banda equivalente de ruido del receptor, que en este caso coincide con el del filtro mostrado en el esquema de la Fig. 10.6.

Cuando no está iluminado, el fotodetector genera una corriente de ruido denominada corriente oscura, $I_{d}$, que contribuye a la varianza de ruido shot como:

$$
\sigma_{s}^{2}=2 q\left(I_{Y}+I_{d}\right) B_{e}
$$

Además, el receptor, como cualquier circuito real, genera una componente de ruido térmico cuya varianza, en términos de corriente, está dada por

$$
\sigma_{T}^{2}=4 k T B_{e} F_{n} / R_{L}
$$

donde $k=1,38 \times 10^{-23} \mathrm{~J} / \mathrm{K}$ es la constante de Boltzmann, $T$ la temperatura equivalente de ruido en kelvin, $F_{n}$ la figura de ruido del amplificador (en caso de no haber, $F_{n}=1$ ) y $R_{L}$ es la resistencia de carga del fotodetector en ohmios. 
La fuente láser, independientemente de la modulación, posee fluctuaciones de intensidad debidas al proceso de emisión espontánea que dan lugar a una componente de ruido denominada ruido de intensidad relativa (Relative Intensity Noise (RIN)) del láser. De esta manera, la potencia óptica a la salida del láser ( $\sin$ modular) se puede escribir de la siguiente manera:

$$
p_{e}(t)=P_{e}+\Delta p_{e}
$$

El segundo término de la expresión anterior es el responsable de generar el RIN en el receptor. En el receptor, la varianza de esta componente de ruido es

$$
\sigma_{R I N}^{2}=P_{\text {rin }} \cdot\left(I_{Y}\right)^{2}=S_{R I N 0} \cdot B_{e}\left(\Re P_{x}\right)^{2}
$$

donde $P_{\text {rin }}$ es una potencia de ruido dada por

$$
P_{\text {rin }} \triangleq \frac{\left\langle\Delta p_{e}^{2}\right\rangle}{P_{e}^{2}}=\int_{-\infty}^{\infty} S_{\text {rin }}(f) d f=S_{R I N 0} \cdot B_{e}
$$

y $S_{\text {rin }}(f)$ es el espectro de potencia del RIN, que en la última igualdad se consideró constante en el ancho de banda $B_{e}$.

De esta manera, la relación señal a ruido y portadora a ruido, respectivamente, pueden expresarse como:

$$
\begin{aligned}
& S N R=\frac{\left(\mathfrak{R} P_{x} M_{0}\right)^{2}\left\langle s^{2}(t)\right\rangle}{\sigma_{s}^{2}+\sigma_{T}^{2}+\sigma_{R I N}^{2}}=\frac{\left(\Re P_{x} M_{0}\right)^{2}\left\langle s^{2}(t)\right\rangle}{2 q\left(I_{Y}+I_{d}\right) B_{e}+4 k T B_{e} F_{n} / R_{L}+S_{R I N 0} \cdot B_{e} \cdot\left(\Re P_{x}\right)^{2}} \\
& C N R=\frac{\frac{1}{2}\left(\Re P_{x} M_{0}\right)^{2}}{\sigma_{s}^{2}+\sigma_{T}^{2}+\sigma_{R I N}^{2}}=\frac{\frac{1}{2}\left(\Re P_{x} M_{0}\right)^{2}}{2 q\left(I_{Y}+I_{d}\right) B_{e}+4 k T B_{e} F_{n} / R_{L}+S_{R I N 0} \cdot B_{e} \cdot\left(\Re P_{x}\right)^{2}}
\end{aligned}
$$

Para evaluar la degradación de la relación señal/ruido debida las pérdidas de la OBFN, en la Fig. 10.9 se muestra un gráfico de la Ec. (10.26) en función de dichas pérdidas y para cuatro valores de $B e: 1 ; 2 ; 3$ y 4 GHz. En la Tabla 10.1 se indican los valores considerados para el resto de los parámetros que intervienen, los cuales corresponden a un enlace típico. 
TABLA 10.1

VALORES TÍPICO DE LOS PARÁMETROS QUE INTERVIENEN EN LA EC. (10.26)

\begin{tabular}{|c|c|c|}
\hline \multirow[t]{4}{*}{ Fuente láser } & Parámetro & Valor \\
\hline & Potencia $\mathrm{Pe}$ & $10 \mathrm{~mW}$ \\
\hline & Longitud de onda $\lambda$ & $1550 \mathrm{~nm}$ \\
\hline & $\operatorname{rin}$ & $-137 \mathrm{~dB} / \mathrm{Hz}$ \\
\hline \multirow[t]{4}{*}{ Fibra óptica } & Parámetro & Valor \\
\hline & Atenuación $\alpha$ & $0,22 \mathrm{~dB} / \mathrm{km}$ \\
\hline & Dispersión $D$ & $17,95 \mathrm{ps} / \mathrm{nm} \cdot \mathrm{km}$ \\
\hline & Longitud $L$ & $7 \mathrm{~km}$ \\
\hline \multirow[t]{3}{*}{ Modulador } & Parámetro & Valor \\
\hline & Pérdidas de inserción $P_{\text {mod }}$ & $6 \mathrm{~dB}$ \\
\hline & Índice de modulación $M_{0}$ & 0,2 \\
\hline \multirow[t]{3}{*}{ Conectores } & Parámetro & Valor \\
\hline & Cantidad $N_{\text {con }}$ & 6 \\
\hline & Pérdidas $P_{c o n}$ & $1 \mathrm{~dB}$ \\
\hline \multirow[t]{3}{*}{ Empalmes } & Parámetro & Valor \\
\hline & Cantidad $N_{e m p}$ & 0 \\
\hline & Pérd idas $P_{e m p}$ & $0,2 \mathrm{~dB}$ \\
\hline \multirow[t]{3}{*}{ Fotodetector } & Parámetro & Valor \\
\hline & Responsividad $\mathfrak{R}$ & $0,7 \mathrm{~A} / \mathrm{W}$ \\
\hline & Corriente oscura $I_{d}$ & $100 \mathrm{nA}$ \\
\hline
\end{tabular}

En la Fig. 10.9 se observa cómo se degrada de la relación señal/ruido al aumentar las pérdidas $I L_{O B F N}$ que aporta la red de conformación de haz a las pérdidas ópticas (Ec. 10.14). También puede verse la influencia del filtro, que debe elegirse de manera que su ancho de banda equivalente, $B e$, sea lo menor posible con el fin de mantener una SNR alta.

Para el enlace simplificado que se presentó, si se establece una cota de $20 \mathrm{~dB}$ como relación seña1/ruido mínima, las pérdidas de la OBFN deberían mantenerse menores a $8,5 \mathrm{~dB}$ para el caso de $B e=1 \mathrm{GHz}$, mientras que si el filtro tiene un ancho de banda equivalente de $4 \mathrm{GHz}$, las pérdidas no deberían superar el valor de 4,48 dB. 


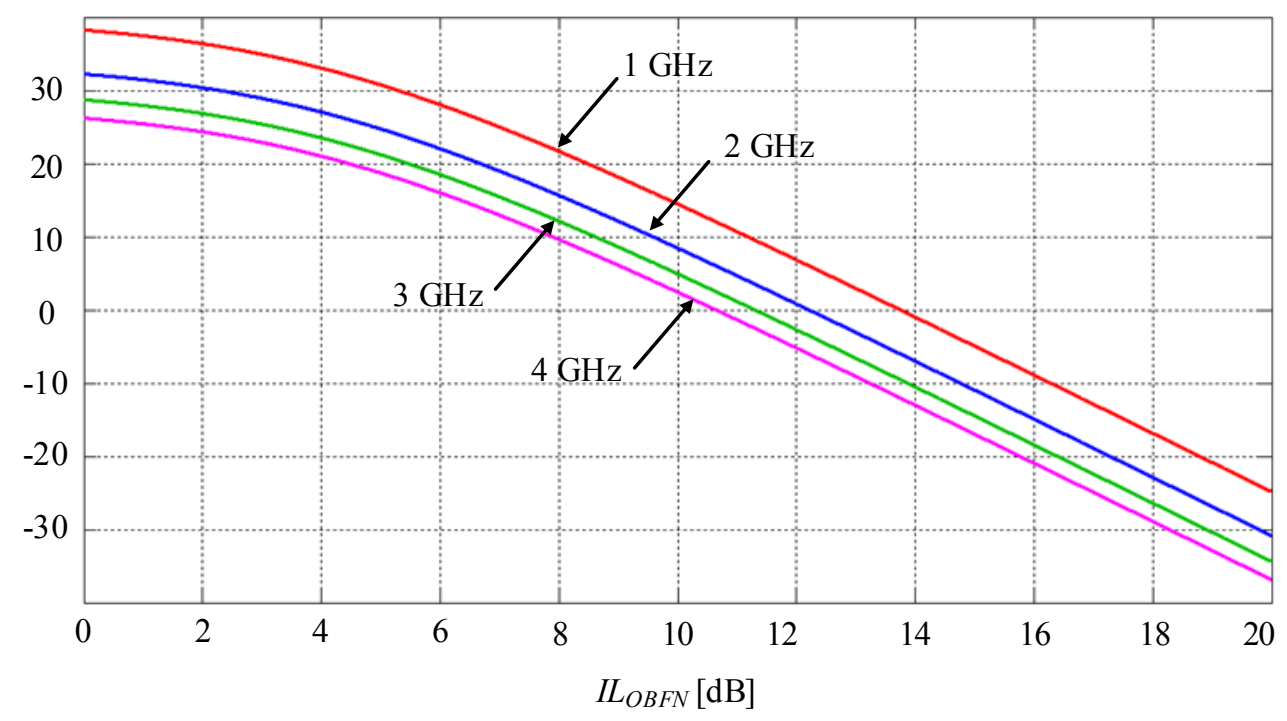

Figura 10.9: Relación señal/ruido en función de las pérdidas de la OBFN con $B e$ como parámetro.

Cabe aclarar que en esta sección no se hizo un análisis cuantitativo del ruido, lo cual está fuera del alcance de este trabajo. Aunque se mencionaron y detallaron los términos de ruido a tener en cuenta, se considera que el sistema trabaja en condiciones de SNR buenas, lo cual es real un muchas aplicaciones.

\subsection{Sistema OBF basado en ORRs}

El sistema OBF que se muestra en la Fig. 10.10 está basado en redes de Bragg uniformes grabadas en fibra (FBGs), y su funcionamiento fue descripto en el Capítulo 1. Como se explicó entonces, las FBGs son dispositivos de fibra óptica que se obtienen realizando un grabado periódico del índice de refracción en el núcleo de la misma, y se comportan como reflectores para cierta longitud de onda, $\lambda_{\mathrm{B}}$, que depende de las características constructivas de la red. En trabajos previos [1], se analizó el patrón de radiación de ese sistema mediante simulaciones numéricas para estudiar la influencia de las posibles desviaciones de algunos parámetros debidas a inexactitudes durante la implementación. Para el análisis se consideraron tres fuentes de error: desviaciones en la longitud de las fibras ópticas, en el grabado de las redes de Bragg, y en la longitud de onda de la fuente de luz. 


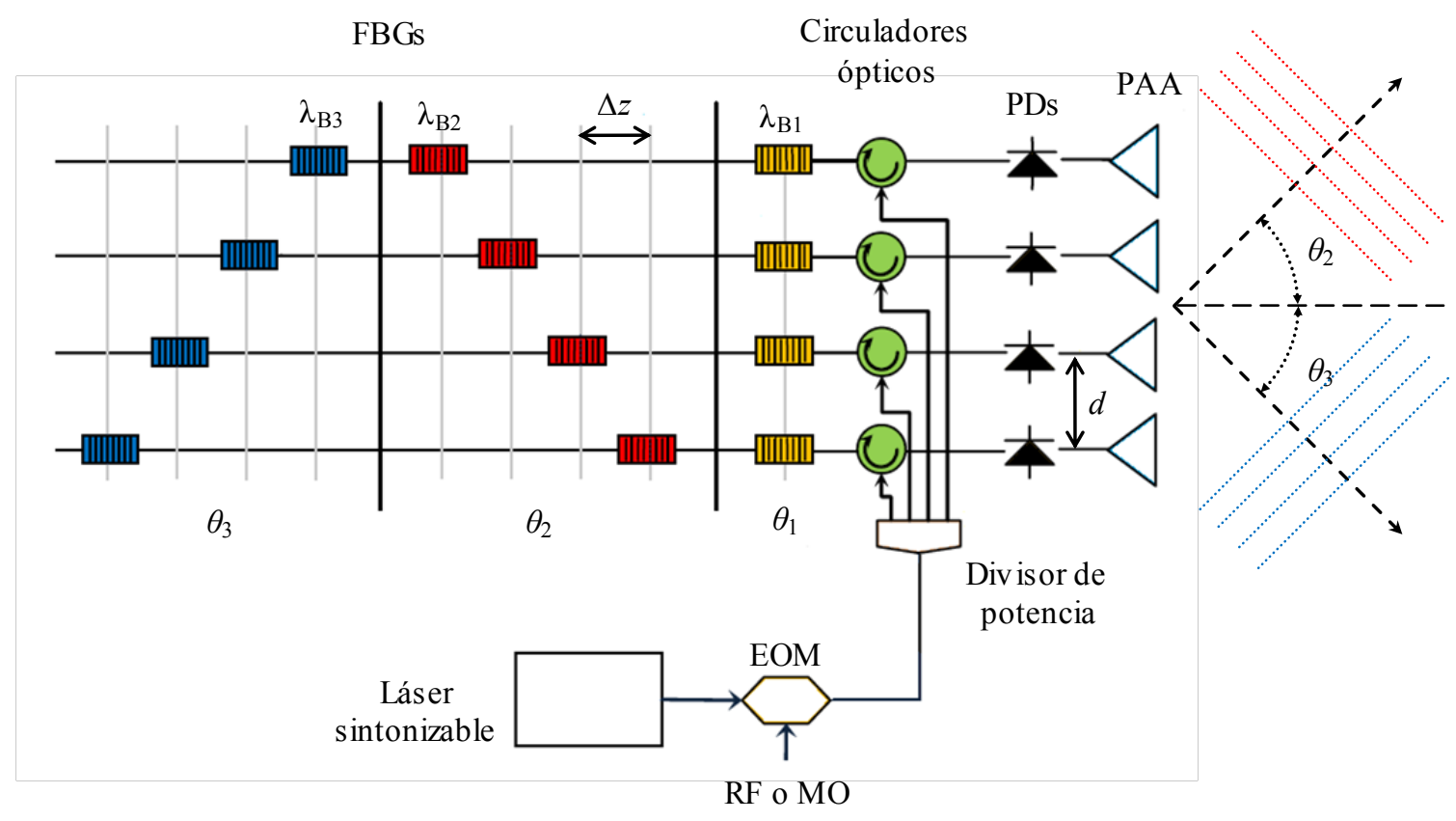

Figura 10.10: Sistema OBF basado en redes de Bragg uniformes.

Los resultados obtenidos revelaron que las desviaciones o inexactitudes que pueden generarse durante el proceso de fabricación del sistema influyen de forma apreciable en el parón de radiación del PAA. Además, al comparar los tres casos, se observó que pequeños errores en la longitud de onda de Bragg de las redes empleadas son los que más distorsión generaron en el patrón, por lo que al implementar este tipo de sistemas OBF debe tenerse especial cuidado en el proceso de fabricación de las FBGs para asegurar que este error esté acotado.

Los Optical Ring Resonators (ORRs) estudiados a lo largo de los capítulos anteriores, además de permitir generar retardos controlados en forma continua, y por lo tanto ángulos de direccionamiento también continuos, permiten la fabricación de líneas de retardo integradas, de dimensiones mucho menores a las del ejemplo anterior, lo que hace al sistema más robusto y fácil de estabilizar.

En esta sección se analiza un sistema conformador de haces que emplea líneas de retardo basadas en ORRs conectados en cascada y que utiliza la estrategia de control presentada en el Capítulo 9. 


\section{Descripción del sistema}

La Fig. 10.11 muestra un esquema simplificado del sistema propuesto, que consta de una fuente láser (LD), un divisor de potencia, cuatro líneas de retardo compuestas por cinco ORRs en cascada cada una más un amplificador EDFA, un fotodetector (PD) seguido de un filtro por cada línea y cuatro antenas isotrópicas ideales. Un sistema real podría incluir un esquema de modulación que incluya algún filtro para implementar, por ejemplo, modulación BLU (Banda Lateral Única) [2,3]. Con el fin de observar únicamente el efecto de los retardos en el patrón de radiación, en el siguiente análisis se considerará que las amplitudes de las señales que alimentan el arreglo de antenas se encuentran ecualizadas. Por otro lado, el esquema de modulación empleado no influye en este análisis.

Las características particulares del sistema propuesto son las siguientes:

La portadora óptica tiene una longitud de onda $\lambda$ cercana a los $1550 \mathrm{~nm}$, y la señal de MO que la modula es de $10 \mathrm{GHz}\left(\lambda_{\mathrm{MO}}=0,03 \mathrm{~m}\right)$. Las antenas del PAA están separadas entre sí una distancia $d=\lambda_{\mathrm{MO}} / 2=0,015 \mathrm{~m}$, y las correspondientes líneas de retardo están implementadas mediante ORRs integrados en dióxido de silicio $\left(\mathrm{SiO}_{2}\right)$ y nitruro de silicio $\left(\mathrm{Si}_{3} \mathrm{~N}_{4}\right)$ de $6 \mathrm{~mm}$ de radio, como los que se estudiaron a lo largo de este trabajo.

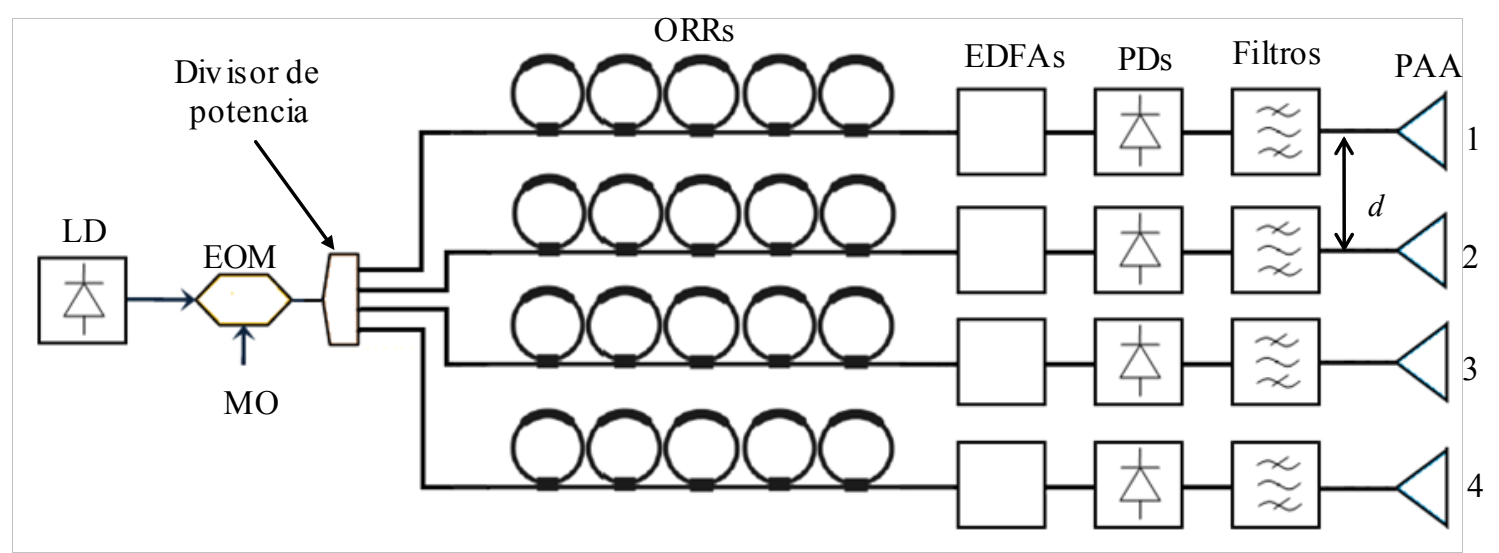

Figura 10.11: Esquema simplificado de un OBF basado en ORRs. 


\section{Aná lis is del patrón de radiación}

Como se mencionó en el Capítulo 1, la Ec. (10.27) determina el retardo relativo que debe existir entre las señales de dos antenas adyacentes para direccionar el haz hacia un cierto ángulo $\theta$.

$$
\Delta t=\frac{d}{c} \cdot \operatorname{sen}(\theta)
$$

Para este análisis, se eligió en forma arbitraria un ángulo de $-45^{\circ}(\Delta t=35,35 \mathrm{ps})$ y, teniendo en cuenta el menor retardo que se puede implementar con cada línea $\left(5 \mathrm{~T}_{\mathrm{RT}}=929,9 \mathrm{ps}\right)$ más un margen de seguridad de $0,3 \mathrm{~T}_{\mathrm{RT}}$, los retardos absolutos que se deben implementar en cada una resultan:

- Línea 1: $\tau_{1}=1091,8 \mathrm{ps}$

- Línea 2: $\tau_{2}=1056,4 \mathrm{ps}$

- Línea 3: $\tau_{3}=1021,1 \mathrm{ps}$

- Línea 4: $\tau_{4}=985,7 \mathrm{ps}$

A continuación, se utilizó la estrategia presentada en el Capítulo 9 para implementar los retardos medios con los valores correspondientes en cada línea de retardo. Los retardos resultantes se muestran en función de la frecuencia en la Fig. 10.12.

Para analizar la influencia que las variaciones del retardo dentro del ancho de banda tienen sobre el patrón de radiación, se seleccionaron distintas frecuencias dentro del mismo y se generó en cada caso un patrón con los valores de retardo correspondientes. En particular, se eligieron veinte frecuencias equiespaciadas, como se muestra en la Fig. 10.12, donde las líneas verticales rojas indican las frecuencias seleccionadas. Un acercamiento permite ver los puntos de retardo seleccionados en el caso de la línea 1 y la variación de los mismos debida al ripple.

Recordando que, en el gráfico, la frecuencia indicada como $0 \mathrm{GHz}$ en el eje horizontal corresponde a la frecuencia de MO portadora de señal, y teniendo en cuenta la simetría de los retardos implementados con respecto a ella, sólo se calcularon los patrones de radiación correspondientes a frecuencias positivas, ya que los que se obtendrían con las frecuencias negativas resultarían idénticos. 


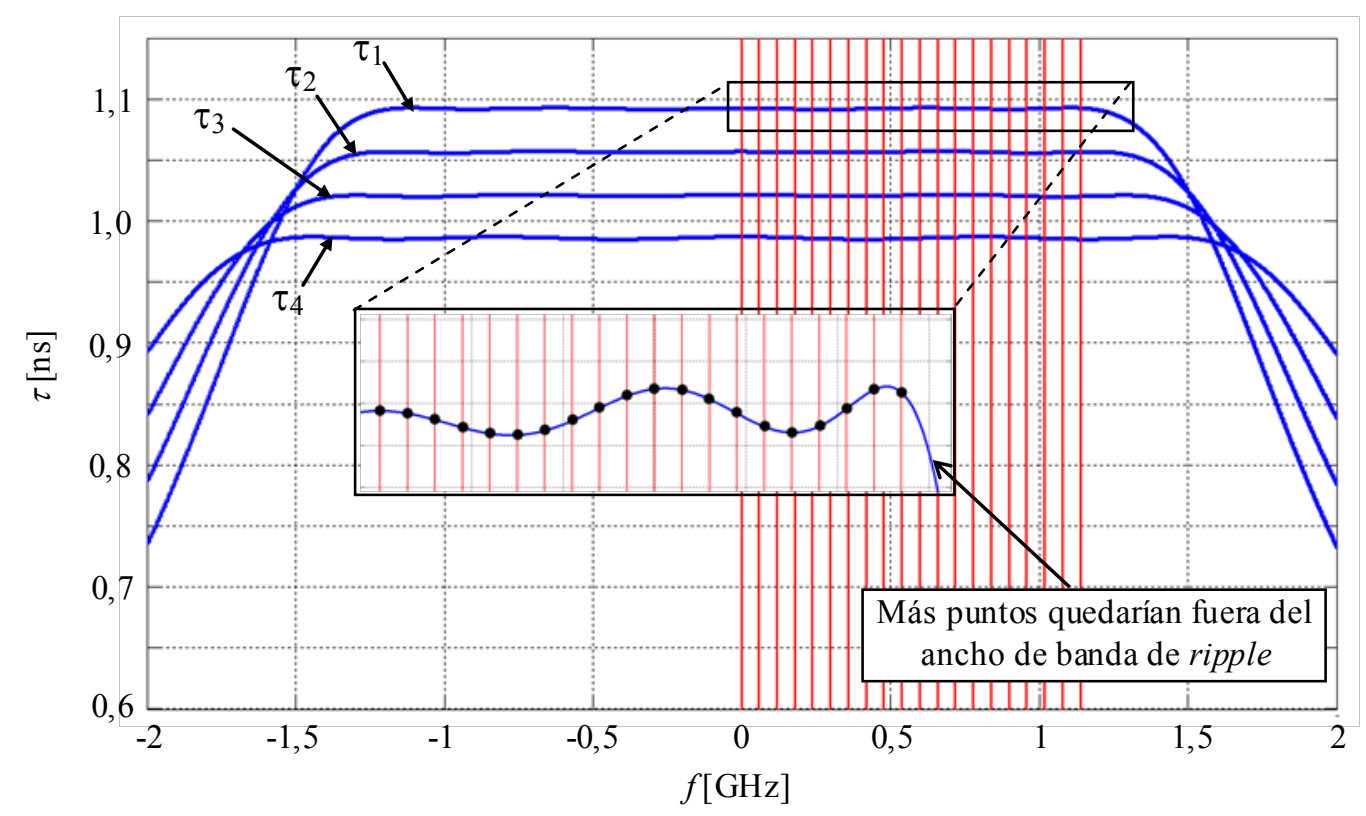

Figura 10.12: Retardos de cada rama del sistema y selección de puntos.

De esta manera, para cada una de las frecuencias indicadas se determinan cuatro valores de retardo, uno para cada línea, que idealmente deberían direccionar el patrón de radiación con el ángulo deseado $\left(-45^{\circ}\right)$ pero que debido al ripple de los retardos puede exhibir desviaciones.

Para efectuar las simulaciones numéricas se utilizaron dos herramientas de software: Optiwave Optisystem ${ }^{\circledR}$ y Matlab ${ }^{\circledR}$. La primera es una herramienta de diseño y simulación de sistemas, enlaces y redes de comunicaciones ópticas, mientras que Matlab ${ }^{\circledR}$ es una herramienta de software matemático ampliamente utilizada en ingeniería. 
TABLA 10.2

CARACTERÍSTICAS DE LOS COMPONENTES DEL SISTEMA QUE SE MUESTRA EN LA FIG. 10.13

\begin{tabular}{|c|c|c|}
\hline Fuente láser & Parámetro & Valor \\
\hline & Potencia $P e$ & $10 \mathrm{~mW}$ \\
\hline & Longitud de onda $\lambda$ & $1550 \mathrm{~nm}$ \\
\hline & Ancho de línea & $0,1 \mathrm{MHz}$ \\
\hline \multirow[t]{4}{*}{ Generador sinusoidal } & Parámetro & Valor \\
\hline & Frecuencia $f_{\mathrm{MO}}$ & $10 \mathrm{GHz}$ \\
\hline & Amplitud & 1 a.u. \\
\hline & Fase & $0^{\circ}$ \\
\hline \multirow[t]{2}{*}{ Modulador MZI } & Parámetro & Valor \\
\hline & Tensión de modulación & $1,5 \mathrm{~V}$ \\
\hline \multirow[t]{2}{*}{ Divisor de potencia } & Parámetro & Valor \\
\hline & Pérdidas de inserción & $0,2 \mathrm{~dB}$ \\
\hline \multirow[t]{4}{*}{ Fibra óptica } & Parámetro & Valor \\
\hline & Atenuación $\alpha$ & $0,22 \mathrm{~dB} / \mathrm{km}$ \\
\hline & Dispersión $D$ & $17,95 \mathrm{ps} / \mathrm{nm} \cdot \mathrm{km}$ \\
\hline & Longitud $L$ & $0,05 \mathrm{~km}$ \\
\hline \multirow[t]{4}{*}{ Fotod iodos PIN } & Parámetro & Valor \\
\hline & Responsividad $\mathfrak{R}$ & $0,9 \mathrm{~A} / \mathrm{W}$ \\
\hline & Corriente oscura $I_{d}$ & $0,5 \mathrm{nA}$ \\
\hline & Ruido térmico & $1 \times 10^{-22} \mathrm{~W} / \mathrm{Hz}$ \\
\hline \multirow[t]{5}{*}{ Filtros } & Parámetro & Valor \\
\hline & Tipo & Bessel \\
\hline & Orden & 4 \\
\hline & Pérdidas de inserción & $1,5 \mathrm{~dB}$ \\
\hline & Frecuencia de corte & $0,75 \cdot f_{\mathrm{MO}}$ \\
\hline
\end{tabular}

Primero se implementó en Optisystem® el sistema que se muestra en la Fig. 10.13, que está compuesto por una fuente láser, un generador sinusoidal, un modulador MachZehnder, un divisor de potencia con cuatro salidas, y cuatro enlaces de fibra óptica con un fotodetector, un filtro y un osciloscopio cada uno. Las características de los componentes utilizados se listan en la Tabla 10.2. 


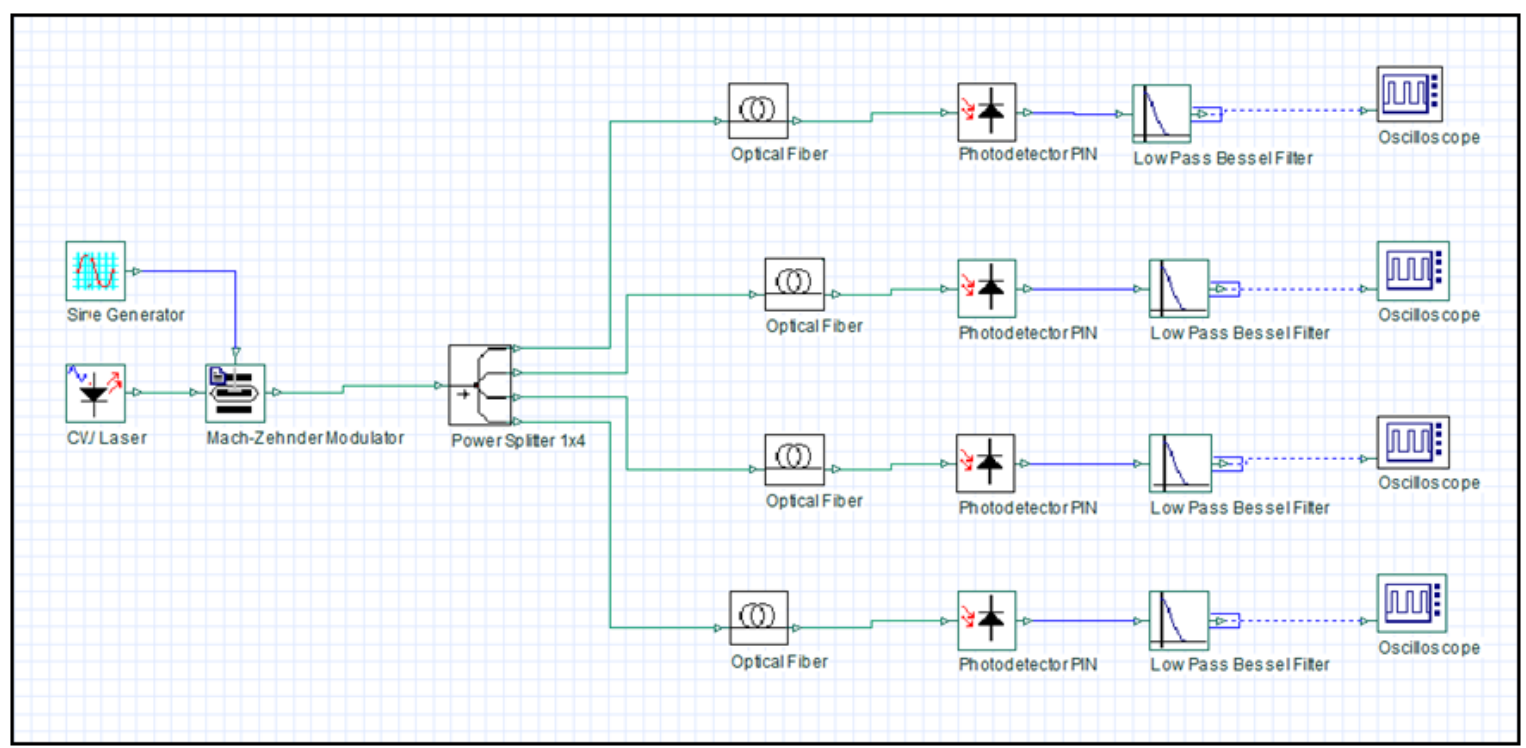

Figura 10.13: Circuito esquemático utilizado para generar las señales de entrada al sistema conformador de haces.

Al finalizar una simulación, los visualizadores (osciloscopios en la Fig. 10.13) contienen la información de las señales que se propagaron a través de los enlaces fotónicos y fueron detectadas. Los datos obtenidos de esta manera fueron exportados para utilizarlos en el código de Matlab ${ }^{\circledR}$ que genera el patrón de radiación mediante las siguientes operaciones:

- Importa las cuatro señales detectadas por los fotodetectores en Optisystem ${ }^{\circledR}$ y les quita el valor medio.

- Para cada ángulo (720 ángulos equiespaciados entre $-90^{\circ} \mathrm{y}+90^{\circ}$ ), determina los retardos que tendrán las señales irradiadas a una distancia de $300 \mathrm{~m}$ del arreglo, debidos a las posiciones de las antenas dentro de éste.

- Calcula la Transformada de Fourier de las cuatro señales.

- Aplica los retardos determinados en la Fig. 10.12, implementados previamente mediante otro programa de Matlab ${ }^{\circledR}$ y calculados para direccionar el haz en un ángulo de $-45^{\circ}$.

- Para cada ángulo, calcula el campo eléctrico resultante y luego la potencia normalizada.

- Presenta en un gráfico polar la potencia normalizada en función delángulo. 
De esta manera, se realizó la simulación de los patrones de radiación correspondientes a cada frecuencia. Los resultados se presentan a continuación, en la Fig. 10.14, donde se muestra en rojo el patrón obtenido para cada frecuencia seleccionada, y en color azul y trazo discontinuo el patrón ideal para $10 \mathrm{GHz}$, que se aprecia mejor al hacer un acercamiento.

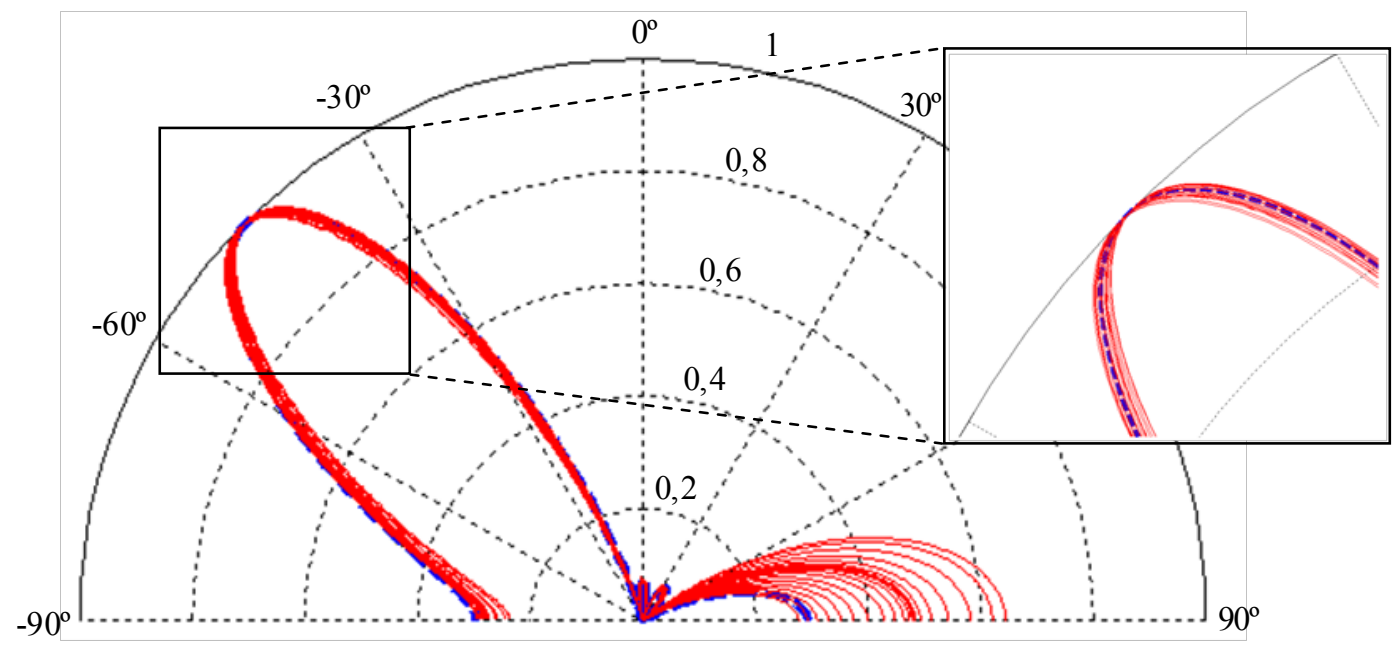

Figura 10.14: Sistema OBF basado en ORRs. Patrones de radiación obtenidos para distintas frecuencias dentro del ancho de banda de ripple.

Como se observa, la potencia irradiada hacia los lóbulos secundarios del patrón exhibe variaciones relativamente grandes. Esto se debe a que son los lóbulos del patrón de interferencia de $\mathrm{N}$ fuentes puntuales, y dependen de la relación entre la longitud de onda de la señal (o, equivalentemente, la frecuencia) y la distancia entre las antenas, como sucede con las rendijas en la experiencia de Young. Esta variación del patrón de radiación es independiente del método que se utilice para controlar los retardos o fases de las señales, ya que se debe a que la señal tiene un cierto ancho de banda. Este efecto no se observaba en los trabajos previos basados en FBGs porque las señales de microondas utilizadas eran de una única frecuencia.

Por otro lado, el lóbulo principal, que se muestra con más detalle en un acercamiento dentro de la misma figura, presenta variaciones del ángulo de direccionamiento, en principio, muy pequeñas. Para cuantificarlas, se puede determinar el ángulo para el cual la potencia irradiada es máxima para cada patrón de radiación simulado. De esta manera se 
encuentra que, en promedio entre todas las simulaciones, este ángulo es de $-45,0^{\circ}$, con una desviación estándar de $0,5^{\circ}$.

A modo de comparación, si se realiza el mismo análisis utilizando desplazadores de fase (phase shifters (PS)) ideales en lugar de true time delays (TTDs), se obtienen los resultados que se exponen en la Fig. 10.15.

Los patrones de radiación que se muestran fueron obtenidos considerando el mismo ancho de banda de trabajo que para el caso anterior y se utilizando la Ec. (10.28) para determinar los desplazamientos de fase correspondientes a cada frecuencia a analizar.

$$
\Delta \varphi=2 \pi \frac{d \cdot f_{M O}}{c} \cdot \operatorname{sen}(\theta)
$$

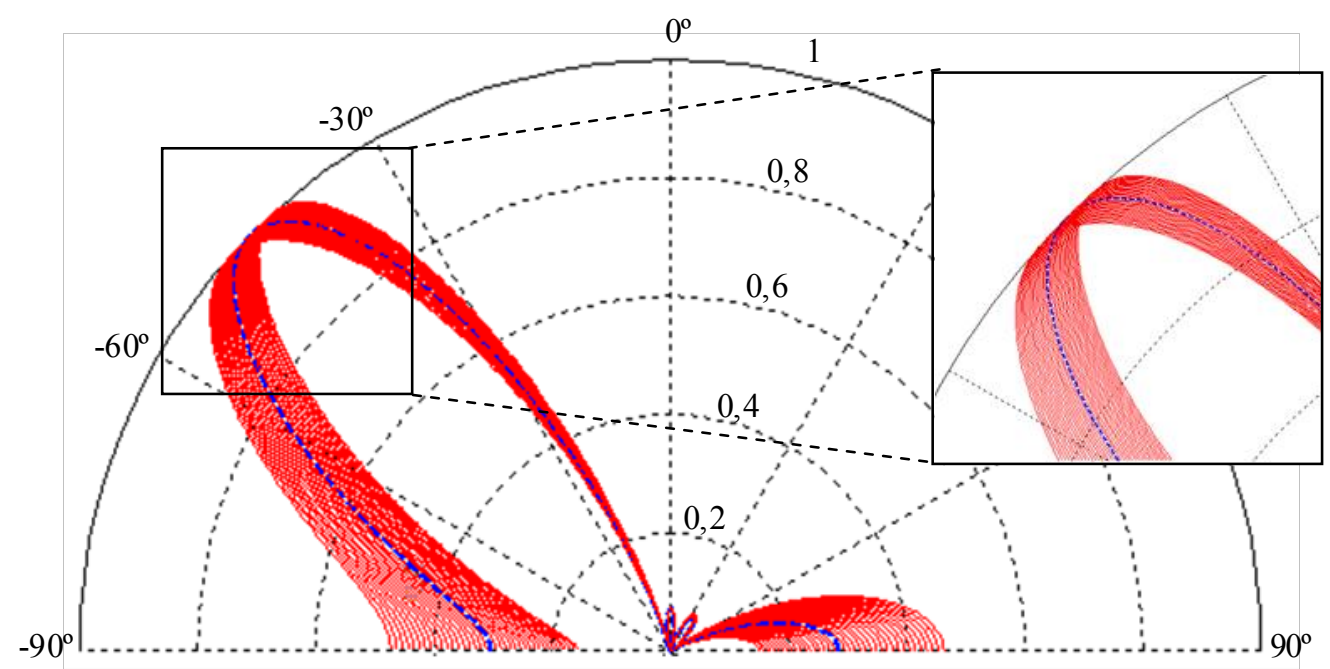

Figura 10.15: Patrones de radiación obtenidos mediante phase shifters para el mismo ancho de banda que en el sistema basado en ORRs.

Como puede apreciarse a simple vista, la variación del ángulo de radiación de la máxima potencia es mayor que en el caso anterior. Esto se ve reflejado en los datos obtenidos, que indican que el ángulo de máxima radiación es, en promedio, de $-45,2^{\circ}$, pero con una desviación estándar de 2,0 $0^{\circ}$. Aunque el valor medio del ángulo direccionado es el mismo que en el sistema basado en ORRs, y coincide con el ángulo deseado, en este caso la variación es cuatro veces mayor. 


\subsection{Conclusiones}

En este capítulo se analizó el desempeño de un sistema conformador de haces basado en los dispositivos ORRs estudiados a lo largo de los capítulos anteriores.

Se efectuaron dos tipos de análisis: en el primero se consideró al sistema compuesto por un conjunto de enlaces ópticos analógicos, uno para cada elemento del arreglo de antenas, y se analizaron sus factores de mérito, mientras que en el segundo se analizó la capacidad del sistema completo para direccionar los haces de microondas.

Del primer análisis se desprende que para las frecuencias de $\mathrm{MO}$ del orden de $10 \mathrm{GHz}$ que se pretenden utilizar, si la longitud de la fibra óptica que conecta la OBFN integrada con el resto de los componentes del enlace no supera los $7 \mathrm{~km}$, las pérdidas debidas a la dispersión cromática se mantienen menores a $1 \mathrm{~dB}$. Por otro lado, para que la relación seña $1 /$ ruido en el lado receptor se mantenga mayor o igual a $20 \mathrm{~dB}$, las pérdidas de la OBFN no deben superar los 4,48 dB en caso de que el filtro del receptor posea un ancho de banda equivalente de ruido de $4 \mathrm{GHz}$. Aunque se consideró un sistema simplificado, estos resultados dan una idea de los valores que pueden esperarse en un caso más complejo.

En el segundo análisis, al estudiar el patrón de radiación que se obtiene para distintas frecuencias dentro de cierto ancho de banda, se encontró que el lóbulo principal se aparta delángulo deseado con una desviación estándar de $0,5^{\circ}$. A modo de comparación, el mismo análisis se realizó para un sistema que emplea phase shifters en lugar de true time delays, y en ese caso las variaciones del ángulo de direccionamiento resultaron cuatro veces mayores. Esto se debe a que, como indica la Ec. (10.28), el ángulo de direccionamiento depende de la frecuencia. Por lo tanto, es evidente que esta situación empeora al aumentar el ancho de banda de la señal a transmitir.

Estos resultados muestran la ventaja de implementar retardos reales para realizar la conformación de haces en lugar de utilizar desplazamientos de fase. Además, la implementación propuesta, basada en ORRs, permite realizar la integración de la mayor parte del sistema OBF, con las ventajas de reducción de espacio y peso. También, como quedó demostrado, mediante la elección de una adecuada estrategia de control se pueden generar retardos con variaciones mínimas dentro del ancho de banda, lo que se traduce en desviaciones delángulo de direccionamiento relativamente pequeñas. 


\section{Referencias}

[1] P. A. Costanzo Caso, S. Rabal, E. Paulucci, A. Giordana, y L. A. Bulus Rossini, "Practical impairments in FBG-based true time delays," Latin America Optics \& Photonics Conference (LAOP), Sao Sebastiao, Brasil, 2012, 1-3.

[2] A. Meijerink, C. G. H. Roeloffzen, L. Zhuang, D. A. I. Marpaung, R. G. Heideman, A. Borreman, W. van Etten, "Phased array antenna steering using a ring resonator-based optical beam forming network," Proc. 13th IEEE/CVT Symp. Benelux, Liège, Belgium, 23 Nov. 2006, pp. 7-12.

[3] L. Zhuang, A. Meijerink, C. G. H. Roeloffzen, D. A. I. Marpaung, J. Peña Hevilla, W. van Etten, R. G. Heideman, A. Leinse, M. Hoekman, "Phased Array Receive Antenna Steering System Using a Ring Resonator-Based Optical Beam Forming Network and Filter-Based Optical SSB-SC Modulation," International Topical Meeting on Microwave Photonics (MWP'2007), 3-5 Oct 2007, Victoria, BC Canada, pp. 88-91. 
Heinrich Sebastian Rabal - Líneas de Retardo Real Para Conformación Óptica de Haces de Microondas 


\section{Conclusiones generales}

En el marco de las radiocomunicaciones de banda ancha, en esta tesis se presentó un dispositivo que posee múltiples aplicaciones dentro del campo del procesamiento fotónico de señales de radiofrecuencia y microondas.

Al principio del trabajo se expusieron las causas que motivaron la investigación, las cuales están relacionadas con la necesidad de implementar sistemas de comunicaciones sensibles a la dirección y capaces de operar en grandes anchos de banda para brindar servicios cada vez más requeridos, como internet de alta velocidad y difusión de contenido multimedia.

Luego se abordó la descripción del dispositivo propuesto, sus componentes principales y teoría de funcionamiento. Se obtuvo un modelo matemático y se realizaron simulaciones numéricas que permitieron definir e indicar algunas características importantes de la respuesta, como la frecuencia de resonancia, el valor pico, el rango espectral libre y el ancho a mitad del máximo.

Un análisis más profundo permitió entender el efecto de la variación de cada uno de los parámetros que intervienen en la función de transferencia del dispositivo. De esta manera, se determinaron los parámetros más adecuados para actuar sobre la respuesta del dispositivo y así controlar los retardos, como el factor de acoplamiento entre las guías, $k$, y el desplazamiento de fase adicional, $\phi$, dentro del anillo. En particular, el primero actúa sobre el valor pico, y el segundo sobre la frecuencia de resonancia de la respuesta. El resto de los parámetros que intervienen en el modelo resultaron poco prácticos para controlar las características mencionadas de los retardos, ya sea por su poca influencia o por su dificultad de implementación.

A partir de un estudio más detallado de la variación de la respuesta con el factor de acoplamiento, se demostró la existencia de una condición de acoplamiento crítico que determina dos zonas de trabajo: una zona subacoplada y una sobreacoplada. Dicha condición, como se demostró, está determinada por la relación entre las pérdidas (de la guía de ondas y delacoplador) y el factor de acoplamiento, $k$.

Los retardos generados por el dispositivo en las dos zonas de trabajo tienen características bien diferenciadas. Por un lado, al operar en la zona subacoplada, los 
retardos exhiben una forma anómala, con valores positivos y negativos en una banda estrecha de frecuencias, y esto puede producir la distorsión de la señal que se propaga por el dispositivo Por este motivo, la zona subacoplada no resulta adecuada para implementar retardos de banda ancha. Por otro lado, en la zona de trabajo sobreacoplada, los retardos toman valores positivos para todas las frecuencias y exhiben forma de campana más o menos suave dependiendo del valor del factor de acoplamiento. Esta zona, por lo tanto, sí resulta apropiada para generar grandes retardos controlados, aunque con una relación de compromiso entre el valor pico y el ancho de banda.

Mediante simulaciones numéricas de la propagación de un pulso a través de un ORR en condiciones sobreacoplada, crítica y subacoplada, se verificó el efecto del retardo de grupo en cada caso. De esta manera, se observó en este último caso el efecto del retardo negativo con respecto a la portadora óptica, en forma de adelantamiento aparente del pulso de salida con respecto al pulso de entrada. Esto se debe a una redistribución de la energía que hace que el pico del pulso se vea adelantado, sin violar el principio de causalidad. En base a los resultados observados, se eligió la zona sobreacoplada como zona de trabajo en los análisis posteriores.

A continuación se presentaron e investigaron configuraciones alternativas y distintos tipos de conexión de los ORRs, y se analizaron sus prestaciones relacionadas con la generación de retardos controlados. En particular, se analizaron los ORRs de cuatro puertos, y las conexiones en cascada y en paralelo de ORRs de dos puertos. El estudio de las distintas variantes permitió concluir que para la implementación de retardos reales de banda ancha que puedan luego ser aplicados en un sistema conformador de haz, la configuración de anillos de dos puertos en cascada resulta la más apropiada. Esto se debe a que dicha topología permite generar retardos de valor adecuado (alrededor de $10 \mathrm{~T}_{\mathrm{RT}}$, que en este caso equivale a $0,186 \mathrm{~ns}$ ), con menor variación y sin requerir un control muy complejo.

Una vez adoptada una topología para las líneas de retardo, se abordó el problema que consiste en determinar los valores de los parámetros de control para cada ORR del sistema. En ese sentido, presentó un método de control muy simple que permite conocer dichos valores e implementar retardos con determinadas características de valor medio y ripple. Básicamente, el método consiste en generar tablas de look up a partir de datos obtenidos 
mediante simulaciones numéricas del sistema en condiciones de retardo aproximadamente plano. Las tablas generadas pueden luego ser utilizadas en tiempo real para controlar los ORRs. Además, las curvas generadas durante el procedimiento permiten caracterizar los retardos generados en cuanto a ripple, ancho de banda, etc.

El método propuesto se describió mediante un ejemplo particular, pero puede aplicarse, en teoría, a un sistema similar con cualquier número de resonadores de los que se conozcan sus características constructivas, requiriendo la simulación y caracterización del sistema en cada caso. En la práctica, la complejidad del sistema aumenta con el número de resonadores conectados en cascada y esto hace que para líneas de retardo de más de 5 anillos sea necesario optimizar el proceso iterativo del método. Además, elFSR de los anillos también limita la cantidad de estos que puede utilizarse en cascada sin que las respuestas de dos anillos y períodos consecutivos se superpongan. Para evitar esta superposición, y dependiendo del ancho de banda requerido, se deberá optar por anillos de dimensiones menores, es decir, con mayor FSR.

Se podrían mencionar algunos resultados que dan cuenta de la capacidad de los ORRs y, en particular, de la estrategia de control adoptada. Por ejemplo, el método permite implementar retardos con valor medio entre 1,02 y 2,55 ns con ancho de banda entre 2,85 y $0,82 \mathrm{GHz}$, respectivamente, y siempre con variaciones menores a 2,08 ps.

Hacia el final del trabajo, y una vez establecidas las características de la línea de retardo y su estrategia de control, se procedió a analizar su desempeño dentro de un sistema de OBF básico.

Primero se analizó su influencia en los factores de mérito del enlace óptico analógico que se establece para cada elemento del arreglo de antenas. Así, se determinó que las pérdidas que introduce la línea de retardo óptica en el enlace no deben superar los 4,48 dB en el caso de utilizar un filtro con ancho de banda equivalente de ruido de $4 \mathrm{GHz}$ en el receptor, con el fin de obtener una relación señal/ruido no menor a $20 \mathrm{~dB}$.

Luego se analizó la capacidad del sistema para direccionar un haz de microondas y las posibles desviaciones con respecto al ángulo deseado. La comparación con un sistema implementado mediante dispositivos electrónicos, como son los desplazadores de fase, muestra que la variación del ángulo de direccionamiento es hasta cuatro veces menor al utilizar las líneas de retardo propuestas. 
Por último, cabe destacar que el trabajo desarrollado en esta tesis sirve como punto de partida para el diseño y la implementación de prototipos de laboratorio que permitan caracterizar en profundidad las líneas de retardo investigadas, y así validar los resultados obtenidos. 


\section{Apéndice A - Acoplador direccional}

\section{A.1. Introducción y funcionamiento}

Un acoplador direccional está constituido por dos fibras monomodo cuyos núcleos se aproximan en la región central de manera que la distancia entre los mismos resulte comparable a sus diámetros y de esta forma los modos fundamentales que se propagan en cada uno se superpongan parcialmente en la región del revestimiento entre ambos.

En la Figura A.1 se muestra el esquema de un acoplador direccional (a), y se indican los campos eléctricos de cada uno de sus puertos, así como la longitud de acoplamiento $L$. En (b) se muestra el corte longitudinal de una fibra óptica para mostrar la forma en que se propaga parte del campo eléctrico a través del revestimiento de la misma (campo evanescente).

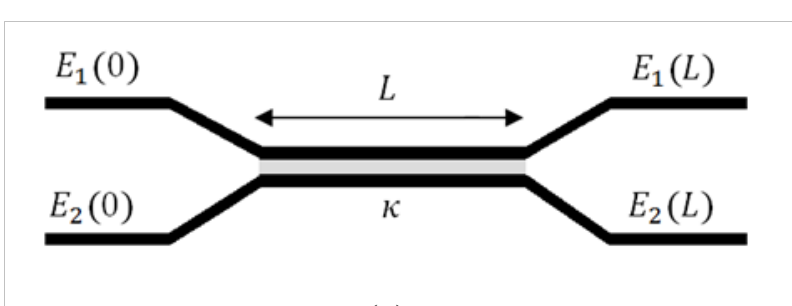

(a)

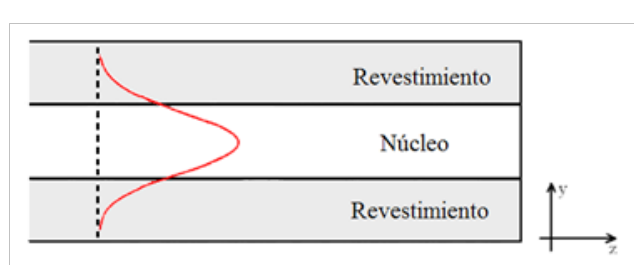

(b)

Figura A.1: (a) Esquema de un acoplador direccional; (b) Campo evanescente.

donde $E_{i}, i=1 ; 2$, es el campo eléctrico en cada uno de los puertos. El coeficiente de acoplamiento $\kappa$ depende de la distancia entre los núcleos, de los parámetros $\mathrm{V}$ y $\Delta$ de la fibra [1], y de la longitud de onda, pero en el presente trabajo se puede considerar constante dentro del rango de trabajo. La potencia en cada puerto de salida está determinada por 
dicho coeficiente de acoplamiento, la longitud $L$ del acoplador, y la potencia en los puertos de entrada.

Las ecuaciones de modos acoplados permiten, partiendo de la ecuación de Helmoltz, describir el comportamiento de los campos eléctricos que intervienen en el acoplador y de esta manera obtener la característica de transferencia de potencia. Por lo general sólo se inyecta luz en uno de los puertos de entrada. En ese caso:

$$
\begin{aligned}
& P_{1}(0)=\left|E_{1}(0)\right|^{2}=P_{0} \\
& P_{2}(0)=\left|E_{2}(0)\right|^{2}=0
\end{aligned}
$$

Por lo tanto, para un acoplador simétrico (aquel en el cual los dos núcleos son idénticos) [1]:

$$
\begin{aligned}
& P_{1}(L)=\left|E_{1}(L)\right|^{2}=P_{0} \cos ^{2}(\kappa L) \\
& P_{2}(L)=\left|E_{2}(L)\right|^{2}=P_{0} \operatorname{sen}^{2}(\kappa L)
\end{aligned}
$$

En la Figura A.2 se muestra la potencia en cada puerto de salida en función de la longitud de acoplamiento $L$ de acuerdo a lo detallado en las Ecs. A3 y A4. Llamando $k=\operatorname{sen}^{2}(\kappa L)$ a la fracción de potencia que se acopla al segundo núcleo, la matriz de transferencia puede escribirse como:

$$
T_{c}=\left[\begin{array}{c}
E_{1}(L) \\
E_{2}(L)
\end{array}\right]=\left[\begin{array}{cc}
\sqrt{1-k} & j \sqrt{k} \\
j \sqrt{k} & \sqrt{1-k}
\end{array}\right] \cdot\left[\begin{array}{l}
E_{1}(0) \\
E_{2}(0)
\end{array}\right]
$$




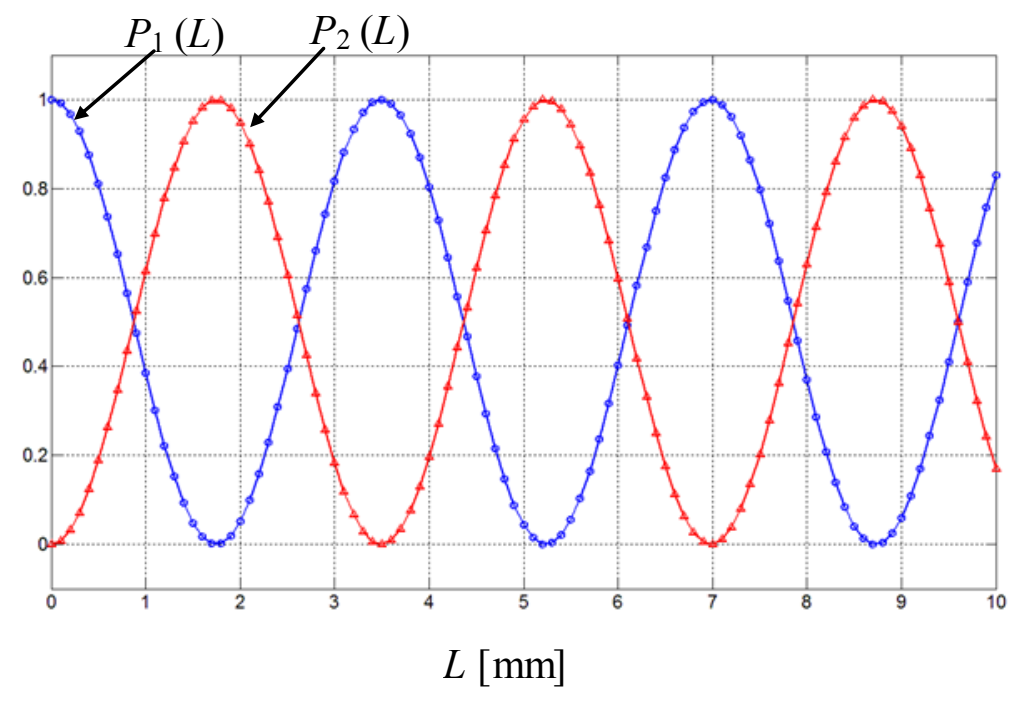

Figura A.2: Potencia de salida normalizada con respecto a $P_{0}$.

En la anterior descripción se consideró un acoplador direccional ideal, en el cual la suma de las potencias a la salida es igual a la potencia de entrada. En realidad esto no es así, sino que existen pérdidas llamadas pérdidas por exceso, $P_{E}$, o en inglés Excess Loss, que se obtienen comparando la potencia entregada a la entrada y la extraída por todas sus salidas.

$$
P_{E}(d B)=10 \cdot \log _{10}\left(\frac{P_{1}(0)}{P_{1}(L)+P_{2}(L)}\right)
$$

Un valor típico para éstas pérdidas es de alrededor de $0,2 \mathrm{~dB}$ en acopladores de fibra. Para tenerlas en cuenta, se introduce el factor $\xi \leq 1$ en la matriz de transferencia:

$$
T_{c}=\left[\begin{array}{l}
E_{1}(L) \\
E_{2}(L)
\end{array}\right]=\xi \cdot\left[\begin{array}{cc}
\sqrt{1-k} & j \sqrt{k} \\
j \sqrt{k} & \sqrt{1-k}
\end{array}\right] \cdot\left[\begin{array}{l}
E_{1}(0) \\
E_{2}(0)
\end{array}\right]
$$

De manera que:

$$
P_{E}(d B)=10 \cdot \log _{10}\left(\frac{1}{\xi^{2}}\right)=-20 \cdot \log _{10}(\xi)
$$

\section{A.2. Referencias}

[1] G. P.Agrawal, Lightwave technology: components and devices, Wiley-Interscience, New Jersey, 2004. ISBN 0-471-21573-2. 
Heinrich Sebastian Rabal - Líneas de Retardo Real Para Conformación Óptica de Haces de Microondas 


\section{Apéndice B - Cálculo del valor pico y FWHM del retardo de grupo para un ORR}

En esta sección se muestra el procedimiento para determinar el valor pico y el FWHM del retardo de grupo normalizado que introduce un ORR, dado por la Ec. (B2).

$$
\begin{gathered}
\tau_{31}(\Omega)=\frac{\xi^{4} a-\xi^{3} \sqrt{a} \cdot \sqrt{1-k} \cdot \cos (\phi+\Omega)}{\xi^{2}(1-k)-2 \xi^{3} \sqrt{1-k} \cdot \sqrt{a} \cdot \cos (\phi+\Omega)+\xi^{4} a} \\
+\frac{\xi \sqrt{1-k} \cdot \sqrt{a} \cdot \cos (\phi+\Omega)-\xi^{2}(1-k) a}{1-2 \xi \sqrt{1-k} \cdot \sqrt{a} \cdot \cos (\phi+\Omega)+\xi^{2}(1-k) a}
\end{gathered}
$$

Teniendo en cuenta que el desplazamiento de fase, $\phi$, no modifica la forma de la respuesta y únicamente actúa como un corrimiento de la frecuencia de resonancia, puede hacerse igual a cero con el fin de simplificar los cálculos.

$$
\begin{gathered}
\tau_{31}(\Omega)=\frac{\xi^{4} a-\xi^{3} \sqrt{a} \cdot \sqrt{1-k} \cdot \cos (\Omega)}{\xi^{2}(1-k)-2 \xi^{3} \sqrt{1-k} \cdot \sqrt{a} \cdot \cos (\Omega)+\xi^{4} a} \\
+\frac{\xi \sqrt{1-k} \cdot \sqrt{a} \cdot \cos (\Omega)-\xi^{2}(1-k) a}{1-2 \xi \sqrt{1-k} \cdot \sqrt{a} \cdot \cos (\Omega)+\xi^{2}(1-k) a}
\end{gathered}
$$

El valor pico, $V_{p}$, puede obtenerse directamente evaluando la Ec. (B3) en $\Omega=0$ :

$$
V_{p}=\tau_{31}(0)
$$


Luego, se pueden hacer los siguientes reemplazos:

$$
\begin{aligned}
& A=\xi^{4} a \\
& B=\xi^{3} \sqrt{a} \cdot \sqrt{1-k} \\
& C=\xi^{2}(1-k) \\
& D=\xi \sqrt{1-k} \cdot \sqrt{a} \\
& E=\xi^{2}(1-k) a \\
& x=\cos (\Omega) \\
& T_{0}=V_{p}=\tau_{31}(0)
\end{aligned}
$$

De esta manera, para calcular elFWHM, se plantea la siguiente ecuación:

$$
\tau_{31}(x)=\frac{A-B \cdot x}{C-2 B \cdot x+\mathrm{A}}+\frac{D \cdot x-E}{1-2 D \cdot x+\mathrm{E}}=\frac{T_{0}}{2}
$$

Operando algebraicamente, se puede llegar a:

$$
R \cdot x^{2}+S \cdot x+T=0
$$

Donde:

$$
\begin{aligned}
& R=\left(-2 \cdot T_{0} \cdot B D\right) \\
& S=\left[(C D+B E-A D-B)+T_{0} \cdot(C D+B+B E+A D)\right] \\
& T=\left[(A-C E)-\frac{T_{0}}{2} \cdot(A+A E+C E+C)\right]
\end{aligned}
$$

Se resuelve (B6) para $x$, y luego se calcula $\Omega$. El valor de $\Omega$ obtenido debe multiplicarse por 2 para obtener elFWHM. 